(C) 2010

Panagiota T. Foteinou

ALL RIGHTS RESERVED 


\title{
A MULTISCALE MODEL FOR THE ASSESSMENT OF AUTONOMIC DYSFUNCTION IN HUMAN ENDOTOXEMIA
}

$$
\text { by }
$$

\section{PANAGIOTA THEODOROS FOTEINOU}

\author{
A Dissertation submitted to the \\ Graduate School-New Brunswick \\ Rutgers, The State University of New Jersey and \\ The Graduate School of Biomedical Sciences \\ University of Medicine and Dentistry of New Jersey \\ in partial fulfillment of the requirements \\ for the degree of \\ Doctor of Philosophy
}

Graduate Program in Biomedical Engineering

written under the direction of

Ioannis $\mathrm{P}$. Androulakis, $\mathrm{PhD}$

and approved by

New Brunswick, New Jersey

May, 2010 
ABSTRACT OF THE DISSERTATION

A Multiscale Model for the Assessment of Autonomic Dysfunction in Human

Endotoxemia

By PANAGIOTA THEODOROS FOTEINOU

Dissertation Director:

Ioannis P. Androulakis, $\mathrm{PhD}$

The overall goal of this dissertation is the development of a semi-mechanistic, physiology-based, multiscale model of human endotoxemia, as a prototype model of acute inflammation in humans that integrates essential regulatory processes across the host from the cellular to the systemic level. The complex and multiplex characteristics of the acute inflammatory response and its complications have been thought to be a leading potential reason for the inability to propose effective clinical intervention strategies. The nature of the response has led researchers to the realization that mathematical models of inflammation offer the opportunity to study the dynamics of interacting components and establish a causal inference relationship through the manipulation of the corresponding dynamic elements.

Driven by the premise to develop in silico methodologies that will enable translational research to elucidate mechanisms by which macroscopic responses, at the physiome level, emerge as the result of propagating information across an intricate web 
of interacting modules, a systems-level modeling approach is developed that addresses the following unique aspects: (i) identification of the essential components characterizing cellular transcriptional dynamics in response to an external perturbation; (ii) reverse engineering of quantifiable representations of these elements exploring the concept of physicochemical and indirect response modeling that connect extracellular signals and intracellular signaling cascades leading to the emergent transcriptional dynamics; and finally (iii) multiscale, physiology-based modeling that quantifies critical aspects of the complex neuro-immune crosstalk while clinical observables are explicitly incorporated to assess systemic abnormalities indicative of the severity of the host.

It is therefore the purpose of this research to demonstrate the feasibility of a relevant human inflammation model that bridges the initiating signal and phenotypic expressions through semi-mechanistic based host response models that include transcriptional dynamics, signaling cascades and physiological components; and to define a notional template for multiscale modeling extendable to a wide range of clinically important conditions. The ultimate deliverable of the proposed research is the multiscale human inflammation model that would allow us to clarify the clinical contexts in which inflammatory dysregulation contributes to morbidity and mortality in acutely stressed patients; thereby advancing the translational potential of systems modeling in clinical research. 


\section{ACKNOWLEDGEMENTS}

I would like to express my deepest gratitude to my thesis advisor Dr. Ioannis P. Androulakis for his supervision, advice, guidance from the very early stage of this research as well as giving me extraordinarily experiences though out the work. Without his generosity and faith in me as a graduate student much of the work presented in this dissertation would not have been possible. Above all and the most needed, he has always been supportive to me when I was in need and he provided me unflinching encouragement to strive for something. He taught me how to ask questions, express my ideas and be persistent to accomplish any goal. His truly scientist intuition and his infectious optimism have exceptionally inspired me to push my limits and enrich my growth as a student, a researcher and a scientist want to be. I am heartily thankful to him more than he knows and I hope to have more opportunities to learn from him in the future.

I have been fortunate to work in an interdisciplinary research project and collaborate with Dr. Calvano and Dr. Lowry, Surgery Department at the Robert Wood Johnson Medical School - UMDNJ to whom I owe great appreciation. I would like to thank both of them for taking the time to discuss with me their insightful comments that definitely influenced the direction of this thesis. I have had unfettered access to their time and knowledge and I sincerely appreciate that. Further, I would like to acknowledge the rest of my committee members - Dr. Moghe and Dr. Yarmush for providing valuable insight on the science as a whole. 
I would like to acknowledge the funding sources which supported me throughout my graduate student career and made this work possible. Suppport for this thesis has been provided primarily by the National Institutes of Health (NIH GM082974) and the National Science Foundation (NSF 0519563). Additional support has been provided by a Busch Biomedical Grant and an NIGMS supplemental award. Special thanks to the Graduate School of New Brunswick for granting me the Bevier Dissteration Fellowship and to Gerondelis Foundation for offering me a scholarship. Further, I would like to acknowledge the following students at Rutgers University: Eric Yang (Rutgers Alumni), Meric Ovacik, Jeremy Scheff, Nguyen Tung, Kaiyuan He, Qian Yang and Pantelis Mavroudis.

Last but not least, I would like to thank my beloved parents, Argiro and Theodoros, for their love, unconditional support and encouragement to pursue my interests through out my entire life as well as my dearest sister, Ioanna, for believing in me. Undoubtedly, I would not have been able to get this degree without the warmth and support of my partner, Spyros Stamatelos who literally helped me along the way constantly reminding me that "it is the journey that matters not the destination". He was the one that had the idea for us of studying abroad (United States) and I am greatly indebted to him for experiencing this "journey". 


\section{Contents}

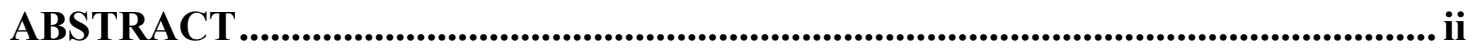

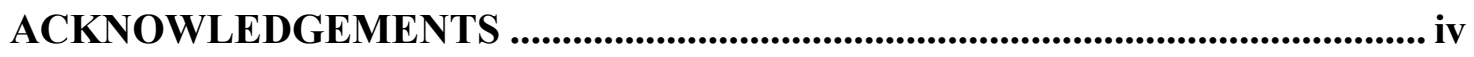

List of Tables ........................................................................................................................... $\mathrm{x}$

List of Figures.................................................................................................................... xi

Chapter 1 ..................................................................................................................... 1

The Problem of Systemic Inflammation ...................................................................... 1

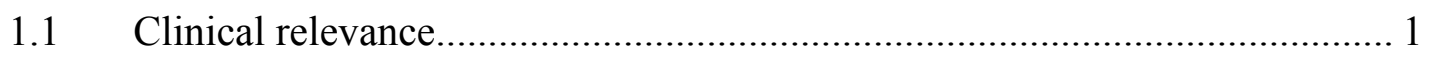

$1.2 \quad$ Neuro-immune crosstalk ................................................................ 2

$1.3 \quad$ Decomplexification and critical illness................................................ 4

1.4 The Pathophysiology of systemic inflammation.......................................... 4

1.5 The era of interdisciplinary and translational research ............................ 7

1.6 Rationale for mathematical modeling ................................................... 9

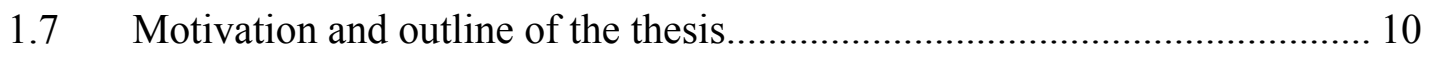

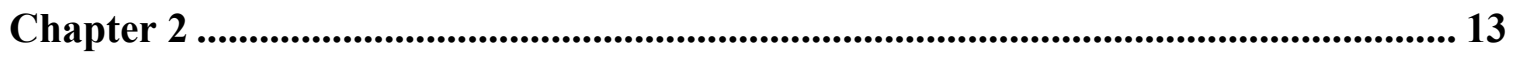

Background .......................................................................................................... 13

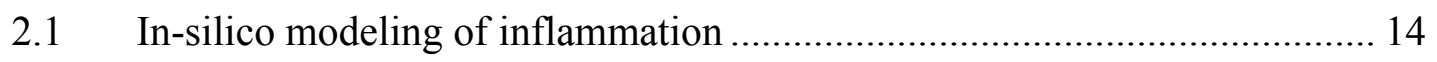

2.2 Clinical models of human endotoxemia ............................................ 22

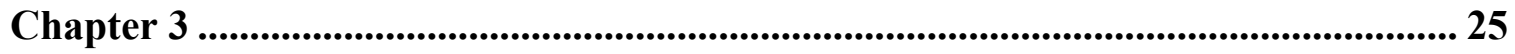

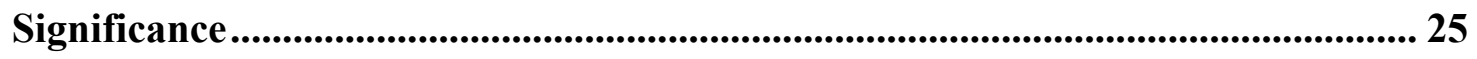


Chapter 4

Transcriptional Analysis and Intrinsic Responses ..................................................... 29

4.1 Extracting essential inflammatory transcriptional responses.................... 31

4.2 Functional characterization of essential responses .................................. 40

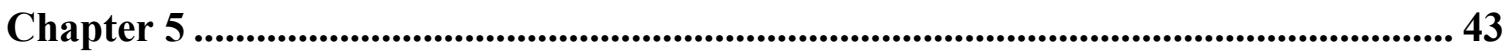

Cellular Level Physicochemical Models of Inflammation........................................ 43

5.1 An indirect response model for human endotoxemia .............................. 44

5.1.1 Elements of an indirect response model of acute human inflammation ... 45

5.1.2 Developing an indirect response model ......................................... 48

5.1.3 Designining and performing in silico experiments ............................ 54

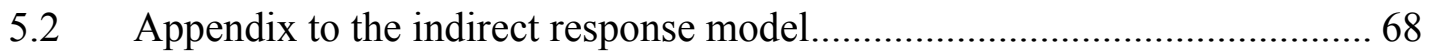

5.3 An NF-kB dependent physicochemical model of systemic inflammation ... 70

5.3.1 Developing physicochemical models of acute inflammation in humans.. 71

5.3.2 Modeling corticosteroid interventions .............................................. 76

5.3.3 Qualitative assessment of NF-kB dependent physicochemical host

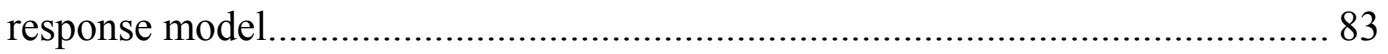

5.3.4 Modulating the progression of an unresolved response......................... 93

5.4 Appendix to the NF-kB dependent physicochemical model .................... 103

5.5 An agent-based model of acute human inflammation ............................ 105

5.5.1 Elements of the agent based host response model of human inflammation 108 
5.5.2 Developing an agent based model of endotoxin induced human

inflammation

111

5.5.3 Qualitative assessment of the model.............................................. 118

5.6 Appendix to the agent-based model of acute human inflammation ........... 133

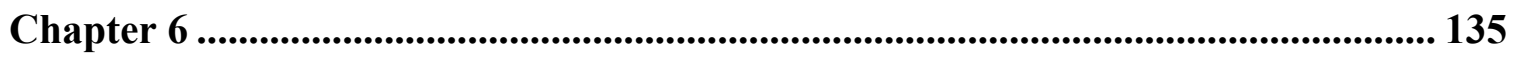

Multiscale Models of Human Endotoxemia ......................................................... 135

6.1 Modeling neuro-endocrine immune system interactions ......................... 137

6.2 A model for the assessment of reduced heart rate variability in human

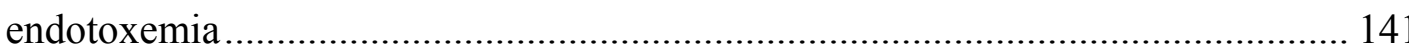

6.2.1 Elements of the multiscale host response model of human inflammation 148

6.2.2 Estimation of relevant model parameters 150

6.2.3 Qualitative assessment of the model

6.3 A physiological model for autonomic control of heart rate in human

endotoxemia.

6.3.1 Developing a semi-mechanistic model for the autonomic control of heart

rate in acute human inflammation.

6.3.2 Elements of the physiology-based model of human endotoxin-induced inflammation 175

6.3.3 Model calibration and validation 177

6.4 Appendix to the multiscale models of human endotoxemia 198

Chapter 7 205

viii 
Conclusions and Future Perspectives............................................................................... 205

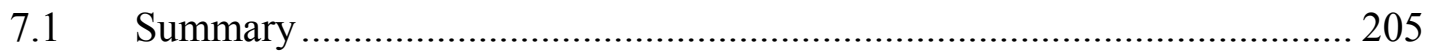

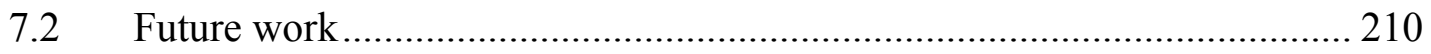

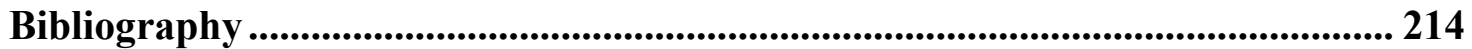

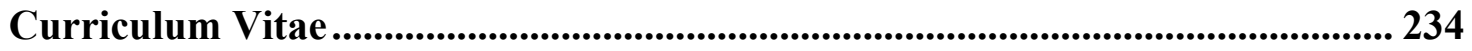




\section{List of Tables}

Table 5.1: The 21 relevant model parameters based on self-limited response data. The

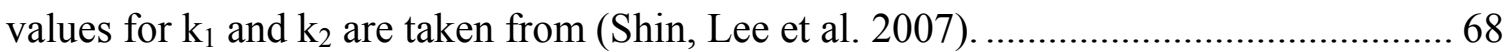

Table 5.2: Estimated values of parameters based on self-limited human data ............. 103

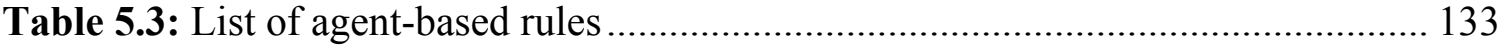

Table 6.1: Estimated values of parameters involved in the neuro-endocrine immune axis.

Table 6.2: Estimated values of parameters related to the autonomic heart rate regulation. 199

Table 6.3: Initial conditions of relevant model components 201

Table 6.4: Statistical assessment of the model in comparison to HRV data presented in

Figure 6.8D 202

Table 6.5: Statistical assessment of the model in comparison to data presented in Figure

6.13C and Figure 6.13D 203 


\section{List of Figures}

Figure 1.1: Hormonal and neural regulation of immunity. The neuro-immune crosstalk is comprised of a descending pathway linking the CNS with peripheral immune tissues and a parallel afferent arm that links the immune system with the CNS. The CNS is a pivotal regulator of the immune response and controls inflammation at multiple levels including activation of the neuroendocrine axis (epinephrine, glucocorticoids) and the vagus nerve (acetylcholine). Adapted from (Matthay and Ware 2004)........................................... 3

Figure 1.2: The complex mechanisms of the host defense system. The initial proinflammatory response may be uncontrolled causing the systemic inflammatory response syndrome (SIRS). A compensatory anti-inflammatory response (CARS) may also be triggered and an imbalance between SIRS and CARS cal lead to multiple organ failure (MOF) (Tetta, Fonsato et al. 2005).

Figure 2.1: (left) Qualitative structure of the four variable model of acute inflammation. Infection $(\mathrm{P})$ triggers inflammation $\left(\mathrm{N}^{*}\right)$ while regulatory mechanisms conceptualized as anti-inflammation $\left(\mathrm{C}_{\mathrm{A}}\right)$ are triggered almost simultaneously. Excessive inflammation results in tissue dysfunction (D); (right) Model dynamics proposed and developed in (Reynolds, Rubin et al. 2006). 16

Figure 2.2: A complex, multivariable mathematical model of systemic inflammation (Clermont, Bartels et al. 2004). 19

Figure 2.3: Electron micrograph of Escherichia Ecoli (E. coli) together with the schematic structure of lipopolysaccharide (LPS, endotoxin). The lipid A component is the primary structure of the toxic center of LPS (Beutler and Rietschel 2003)..... 23 
Figure 2.4: Endotoxin kinetics and mean expression of critical pro- and antiinflammatory components following $2 \mathrm{ng} / \mathrm{kg}$ intravenous bolus of endotoxin. Adapted from (Lowry 2005).

Figure 3.1: The state of a system is expressed in multiple scales of the biological organization. Changes at the genome (low) level lead to modified intracellular signaling which causes changes in cellular behavior and thereby gives rise to perturbations to higher biological scales (tissues, organs, entire organism). Focusing on the cell as the fundamental unit allows us to naturally link molecular reductionism with quantitative

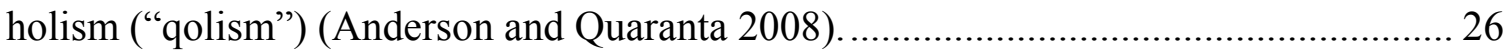

Figure 4.1: Number of probe sets in each expression motif of inflammatory transcriptional signatures of human blood leukocytes. A histogram of hash values is used to visualize the number of probe sets in each micro-cluster. 5,093 probe sets are microclustered to 224 expression motifs. 32

Figure 4.2: Estimated p-value vs. expression motif sizes. Expression motifs with size $\geq$ 58 corresponding to $\mathrm{p}$-value $<1 / 224$ (total number of probe sets in informative motifs). 34 Figure 4.3: Temporal profiles of statistically significant expression motifs. Normalized expression values of motifs with p-value $<0.0045$ (with respect to cluster size) vs. time.

Figure 4.4: Estimation of the Transcriptional State of the System. Distribution of expression values for all genes at each time point $(\mathrm{t}>0 \mathrm{hr})$ versus the baseline distribution $(\mathrm{t}=0 \mathrm{hr})$ 35 
Figure 4.5: Deviation of the Transcriptional State of the system vs. number of expression motifs. The maximum perturbation in the intrinsic dynamics of the system occurs for 3 distinct expression motifs. 37

Figure 4.6: Essential leukocyte transcriptional elements: (red color) Pro-inflammatory response (P), (green color) Anti-inflammatory response (A) and (blue color) Energetic response $(\mathrm{E})$. 39

Figure 4.7: Transcriptional signatures of marker inflammatory genes: (red color) mRNA of IL1B representative of the essential pro-inflammatory response. (green color) mRNA of IL10RB indicative of the late anti-inflammatory response and (blue color) mRNA of NDUFC2 which is considered as the proxy for the energetic component. Data are represented as mean $\pm \mathrm{SEM}$. 42

Figure 5.1: Notional modeling framework of LPS response. Upon binding to its receptor a signaling cascade is activated which leads to the up/down-regulation of numerous proand anti-inflammatory genes. LPS binds to the receptor (R) and forms the complex (LPSR) while it activates the signaling complex (DR*) which indirectly stimulates the production rate $\mathrm{K}_{\mathrm{in}, \mathrm{P}}$ of the pro-inflammatory $(\mathrm{P})$ response. The pro-inflammatory response indirectly stimulates the production rate of the energetic $(E)$ response $\left(K_{i n, E}\right)$ and the production rate of anti-inflammatory $(A)$ response $\left(K_{i n, A}\right)$. The energetic response will stimulate both pro-inflammation and anti-inflammation whilst anti-inflammation will serve as the immunoregulatory component of the system restoring homeostasis intracellularly. 47

Figure 5.2: Mathematical representation of an indirect response model of endotoxininduced human inflammation. 51

xiii 
Figure 5.3: Model Building Results: Dynamic profiles of the elements that constitute the semi-mechanistic model of endotoxin-induced inflammation. Experimentally measured normalized mRNA transcript levels are denoted by symbols $(\bullet)$, solid lines $(-)$ are the

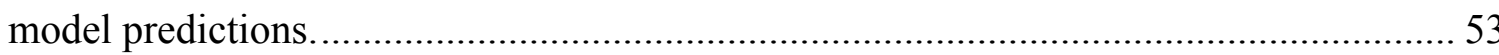

Figure 5.4: Temporal responses of critical inflammatory components for various initial conditions of the inflammatory stimulus. A high concentration of LPS can cause a malfunction in the dynamics of the host response to infection described by an exacerbated inflammatory response (dashed line). Solid lines correspond to self-limited responses, the dashed line represent a predicted unconstrained inflammatory response when the LPS concentration exceeds a critical value. 55

Figure 5.5: Temporal responses of inflammatory components in persistent infectious inflammatory response where the inflammatory stimulus cannot be eliminated responsible for the observed persistence in the dynamic profiles of the inflammatory constituents. Reducing the degradation rate of LPS to half of its initial value the inflammatory stimulus cannot be cleared. 57

Figure 5.6: Temporal profiles of persistent non - infectious inflammation. It is not the infection itself but rather the host response to infection that plays a determinant role in controlling the outcome of an overwhelming inflammatory response. Manipulating the degradation rate of the activated intracellular signaling (i.e. reducing it by $1 / 3$ of its initial value) perturbs the homeostasis of the system. 59

Figure 5.7: Rapid Endotoxin Tolerance: Pre - exposuring the system into a smaller inflammatory insult results in a reduction in the cell capacity to respond to the main endotoxin challenge which is characterized as a short-time attenuation scenario Solid xiv 
line: $\operatorname{LPS}(\mathrm{t}=0 \mathrm{hr})=0.5 \& \operatorname{LPS}(\mathrm{t}=4 \mathrm{hr})=1$ Dashed line: $\operatorname{LPS}(\mathrm{t}=0 \mathrm{hr})=0 \& \operatorname{LPS}(\mathrm{t}=4 \mathrm{hr})$ $=1$.

Figure 5.8: Endotoxin Hypo-responsiveness: Pre-existing infection might cause a profound hypo-responsiveness in system's response to a lethal LPS challenge Solid line: $\operatorname{LPS}(\mathrm{t}=0 \mathrm{hr})=1 \& \operatorname{LPS}(\mathrm{t}=4 \mathrm{hr})=4$ Dashed line: $\operatorname{LPS}(\mathrm{t}=0 \mathrm{hr})=0 \& \operatorname{LPS}(\mathrm{t}=4 \mathrm{hr})=4 \ldots 64$

Figure 5.9: Lethal Potentiation: Successive administration of small doses of endotoxin can lead to an unresolved inflammatory response (due to loss of "regulatory" memory Solid line: $\operatorname{LPS}(\mathrm{t}=0 \mathrm{hr})=1 \& \operatorname{LPS}(\mathrm{t}=0.5 \mathrm{hr})=2$ Dashed line: $\operatorname{LPS}(\mathrm{t}=0 \mathrm{hr})=0 \& \operatorname{LPS}(\mathrm{t}$ $=0.5 \mathrm{hr})=2$. 66

Figure 5.10: Endotoxin-induced intracellular signaling cascade. The inflammatory response is activated when endotoxin is recognized by pathogen recognition receptors that triggers downstream critical signaling modules for the activation of inflammatory relevant transcriptional factors (Annane, Bellissant et al. 2005). 71

Figure 5.11: Dynamic evolution of model elements that constitute the mechanistic pharmacokinetic model of corticosteroids for a single intravenous administration (bolus injection) of $50 \mathrm{mg} / \mathrm{kg}$ and given the parameters and initial conditions extracted from (Jin, Almon et al. 2003). 80

Figure 5.12: Schematic illustration of a reverse engineered model of systemic inflammation. Interacting modules involve the propagation of LPS signaling on the transcriptional response level coupled with the anti-inflammatory effect of corticosteroids. The propagation of LPS signaling involves the interaction of the inflammatory stimulus, LPS with its receptor (R) forming the surface complex (LPSR) which activates IKK activity. The IKK-dependent signal activates the translocation of 
$\mathrm{NF}-\mathrm{kB}\left(\mathrm{NFkB}_{\mathrm{n}}\right)$ through phosphorylation and degradation of its primary inhibitor, IkBa. The nuclear NF-kB (NFkBn) is auto-regulated by its inhibitor protein, IKBa and stimulates the production rate of the pro-inflammatory response $(\mathrm{P})$ while there is certain connectivity among the essential transcriptional signatures (P, A, E). The mRNA of the receptor $(\mathrm{mRNA}, \mathrm{R})$ is stimulated by pro-inflammation $(\mathrm{P})$ and it is translated to the surface protein (R). The corticosteroid intervention envelope consists of the corticosteroid drug $(\mathrm{F})$ which binds to its intracellular receptor $\left(\mathrm{R}_{\mathrm{F}}\right)$ forming the cytosolic complex (FR) that translocates to the nucleus $(\mathrm{FR}(\mathrm{N}))$ and modulates the dynamics of inflammation via an upregulation of anti-inflammatory proteins (IkBa, A) represented by dotted lines. 85

Figure 5.13: Estimation of relevant model parameters. Temporal profiles of the elements that constitute the NF-kB dependent model of endotoxin-induced inflammation. Solid lines $(-)$ correspond to model predictions whilst the symbols $(\bullet)$ denote for the experimentally measured transcriptional signatures.

Figure 5.14: Temporal responses of model elements in a persistent infectious inflammatory response. Reducing the degradation rate of LPS to half of its initial value we simulate the case of an unsuccessful clearance of LPS that accounts for the sustained (aberrant) activity of NF-kB leading to a chronic inflammatory response.

Figure 5.15: Simulation of a knock-out in silico experiment $\left(\mathrm{IkBa}^{-/}, \mathrm{k}_{\mathrm{IkBa}, 1}=0\right)$. Manipulating the model so that there is no de novo transcriptional synthesis of NF-kB inhibitor (IkBa) which is responsible for the absence of NF-kB auto-regulatory feedback loop. Such a scenario accounts for maladapted activity of $\mathrm{NFkB}_{\mathrm{n}}$ that triggers an uncompensated inflammatory response. 90 
Figure 5.16: Pre-existence of pro-inflammatory mediators may enhance abnormally the intracellular signaling through IKK. Such a response leads to an unconstrained activity of NFkBn that drives downstream a persistent pro-inflammatory response which cannot be counter-regulated by the anti-inflammatory arm of the host defense system. Such a mode of dysregulation is simulated by manipulating the zero production rate of proinflammation $\left(\mathrm{K}_{\mathrm{in}, \mathrm{P}}\right)$ so that $\mathrm{K}_{\mathrm{in}, \mathrm{P}}$ (unconstrained response) $\sim 2 * \mathrm{~K}_{\mathrm{in}, \mathrm{P}}$ (self-limited response).

Figure 5.17: Exploring the mode of corticosteroid action in enhancing the transcriptional synthesis of $\mathrm{IkBa}$ which is illustrated by the solid arrow. An i.v. injection of the corticosteroid drug administered concomitantly with endotoxin $\left(t_{\text {in }}=0 \mathrm{hr}\right)$ suffices to reverse (prevent) the lethal effect of a high dose of endotoxin. Solid lines $(-)$ correspond to the inflammatory resolution due to the corticosteroid injection at $\mathrm{t}=0 \mathrm{hr}$ while dashed lines simulate the progression of inflammation in response to a high concentration of LPS (i.e. $\operatorname{LPS}(\mathrm{t}=0 \mathrm{hr})=4)$ 95

Figure 5.18: Exploring the effect of steroid infusion initiated simultaneously with LPS injection $\left(\mathrm{t}_{\mathrm{in}}=0 \mathrm{hr}\right)$ and continued for $6 \mathrm{hr}\left(\mathrm{t}_{\text {stop }}=6 \mathrm{hr}\right)$ after LPS exposure in modulating the progression of unremitting inflammation evoked by high LPS dose. Solid lines (-) correspond to the inflammatory resolution due to the corticosteroid infusion while dashed lines simulate the progression of aberrant inflammation in response to high LPS challenge. 96

Figure 5.19: Hypercortisolemia for $6 \mathrm{hr}$ prior to LPS challenge $\left(\mathrm{t}_{\mathrm{in}}=-6 \mathrm{hr}\right)$. The system is pre-exposed for $6 \mathrm{hr}$ to a continuous infusion of corticosteroids while it is continued for 
another $6 \mathrm{hr}$ after the endotoxin challenge $\left(\mathrm{t}_{\text {stop }}=6 \mathrm{hr}\right)$. Such an intervention "reprograms" the dynamics of the system modulating the effect of a high LPS concentration 97

Figure 5.20: Pre-exposure the system into hypercortisolemia which is assumed to modulate the dynamics of IL-10 signaling (A). Such intervention strategy is initiated $18 \mathrm{hr}$ before the endotoxin challenge and continued for $6 \mathrm{hr}$ while it does not have a profound effect in the dynamic state of the system where the progression of an unresolved inflammation (- lines) continues after the termination of steroid infusion (represented by overlap between solid and dashed lines).

Figure 5.21: Explore the effect of corticosteroids at multiple drug doses initiated at $t=$ $1 \mathrm{hr}$ and continued for $6 \mathrm{hr}$ after the endotoxin challenge priming the production rate of IL10 signaling (A component) illustrated by green arrow. Solid lines characterize a resolution in the progression of systemic inflammation whereas dashed and dotted lines correspond to lower drug doses that cannot sufficiently reverse the progression rate of an aberrant inflammation. All the active signals, FR(N)norm, have been normalized with respect to the lowest drug dose, $\mathrm{F} 0=20 \mathrm{ng} / \mathrm{mL}$ (represented by dotted lines). 100

Figure 5.22: Schematic illustration of elements and interactions involved in the agent based model of endotoxin induced inflammation. 109

Figure 5.23: A self-limited inflammatory response ( $\operatorname{LPS}(0)=350$ units). Temporal profiles of essential components that constitute the agent based model resolved within $24 \mathrm{hr}$. 119

Figure 5.24: Temporal responses of an unresolved inflammatory response due to high LPS concentration. A high concentration of LPS $(\operatorname{LPS}(0)=750)$ can cause a malfunction in the dynamics of the host leading to an exacerbated inflammatory response (solid lines). xviii 
Dashed lines refer to the implications of high concentration of LPS as simulated by our deterministic (ODE) approach. For the purpose of comparing the simulated output between the $\mathrm{ABM}$ and the $\mathrm{ODE}$ model, all responses are normalized so that numerically they range between $(0,1)$. 121

Figure 5.25: Temporal responses in a persistent infectious inflammatory response. Solid lines correspond to $\operatorname{LPS}(\mathrm{t}=0 \mathrm{hr})=1000$ which accounts for a prolonged inflammatory activity causing a malfunction in LPS clearance rate. Dashed lines refer to equation-based model predictions for the case of a persistent infectious challenge which can be achieved by manipulating the first order degradation rate of LPS as discussed in the deterministic approach (Section 5.3). The output of both modeling approaches is normalized so that numerically it ranges between $(0,1)$. 123

Figure 5.26: Endotoxin tolerance scenario. Pre-existing infection might cause a profound reduction in cell's capacity (hypo-responsiveness) to respond in the main endotoxin challenge. Solid line: $\operatorname{LPS}(\mathrm{t}=0 \mathrm{hr})=750$. Dotted line: $\operatorname{LPS}(\mathrm{t}=0 \mathrm{hr})=100 \& \operatorname{LPS}(\mathrm{t}=8 \mathrm{hr})$ $=650$. 126

Figure 5.27: Lethal potentiation. Successive administration of small doses of endotoxin can lead to an unresolved inflammatory response. Solid line: $\operatorname{LPS}(\mathrm{t}=0 \mathrm{hr})=350$. Dotted line: $\operatorname{LPS}(\mathrm{t}=0 \mathrm{hr})=100 \& \operatorname{LPS}(\mathrm{t}=2 \mathrm{hr})=250$. 128

Figure 5.28: (left) Pre-existence of inflammatory mediators (TNF-a) may enhance abnormally the intracellular signaling amplifying IKK activity. Such response leads to an aberrant inflammatory response which cannot be counter-regulated by the antiinflammatory arms of the system. Such a mode of dysregulation is simulated by concomitant exposure of the system to TNF-a and bacterial infection (LPS): LPS( $\mathrm{t}=0 \mathrm{hr})$ 
$=350 \& \mathrm{TNF}-\mathrm{a}(\mathrm{t}=0 \mathrm{hr})=300$; (right) Exploring the effect of an intervention (antiinflammatory) strategy that inhibits IKK activity. Such scenario is simulated by administering $\operatorname{LPS}(\mathrm{t}=0 \mathrm{hr})=750$ and IKK inhibitors, IKK inhibitors $(\mathrm{t}=0 \mathrm{hr})=400$ (dashed lines).

Figure 6.1: Basic topological interactions composing the multi-level model of endotoxin induced human inflammation. At the cellular level, interacting components involve the propagation of LPS signaling on the transcriptional response level $(\mathrm{P}, \mathrm{A}, \mathrm{E})$ through the activation of endotoxin signaling receptor (R) and elementary signaling pathways (NF-kB signaling module). At the level of circulating hormones, essential modules are associated with the release of endocrine stress hormones from neuroendocrine axis (HPA, SNS) coupled with their anti-inflammatory influence on the host. The dynamics of cortisol and epinephrine signaling involve components interacting at the cellular level. At the systemic level, physiologic deterioration of the host is quantified by HRV. 149

Figure 6.2: Basic topological interactions composing the multi-level model of endotoxin induced human inflammation. At the cellular level, interacting components involve the propagation of LPS signaling on the transcriptional response level $(\mathrm{P}, \mathrm{A}, \mathrm{E})$ through the activation of endotoxin signaling receptor (R) and elementary signaling pathways (NF-kB signaling module). At the level of circulating hormones, essential modules are associated with the release of endocrine stress hormones from neuroendocrine axis (HPA, SNS) coupled with their anti-inflammatory influence on the host. The dynamics of cortisol and epinephrine signaling involve components interacting at the cellular level. At the systemic level, physiologic deterioration of the host is quantified by HRV. 
Figure 6.3: Estimation of relevant model parameters intending to reproduce available experimental data associated with transcriptional signatures (A) and plasma counterregulatory hormones including epinephrine (EPI) and cortisol (F) as well as clinical data (HRV). Solid lines (-) correspond to model predictions under conditions of low-dose endotoxin while the symbols $(\bullet)$ refer to experimental data expressed as mean \pm SEM. The initial condition of endotoxin $(\operatorname{LPS}(\mathrm{t}=0 \mathrm{~h})=1)$ refers to LPS concentration relative to 2-ng/kg body weight. 153

Figure 6.4: Simulation of an unresolved inflammatory response due to high endotoxin concentration $(\operatorname{LPS}(t=0 h)=4)$. Such high concentration of LPS (four times greater than the nominal value) deregulates the NF-kB signaling module giving rise to an unconstrained immune response followed by abnormal hormonal responses that macroscopically are translated into diminished physiologic variability 155

Figure 6.5: Simulating the effect of acute epinephrine infusion $\left(\mathrm{w}_{\mathrm{EPI}, \mathrm{ex}}=1\right)$, which is initiated $3 \mathrm{~h}$ prior to the main endotoxin challenge $(\operatorname{LPS}(\mathrm{t}=0 \mathrm{~h})=4)$ and continued for $6 \mathrm{hr}$ after LPS $\left(\mathrm{R}_{\mathrm{in}, \mathrm{EPI}}=15\right)$, under conditions of severe inflammation. Dashed and solid lines represent the progression of a balanced (due to system's pre-exposure into epinephrine infusion) and unconstrained inflammatory response (due to high inflammatory challenge, $\operatorname{LPS}(\mathrm{t}=0 \mathrm{~h})=4)$ respectively. Acute pre-exposure of the host to epinephrine attenuates the aberrant pro-inflammatory response (P) induced by high LPS concentration which allows for recovery in HRV dynamics (restoration in autonomic balance).

Figure 6.6: Explore the effect of low-dose steroid administration initiated 6h prior to endotoxin challenge (dashed lines) while continued for another $6 \mathrm{~h}$ after LPS $\left(\mathrm{w}_{\mathrm{Fex}}=1\right)$ under conditions of high LPS concentration (solid lines). Solid lines simulate the xxi 
progression of a systemic inflammatory response syndrome (due to high LPS concentration, $\operatorname{LPS}(\mathrm{t}=0 \mathrm{~h})=4$ ) while dashed lines reflect the protective effect that can be exerted by hormonal(steroid) replacement therapy. The acute pre-exposure of the host to exogenous cortisol dampens the excessive pro-inflammatory effects induced by high LPS concentration which allows for restoration in autonomic balance (HRV).

Figure 6.7: Explore the effect of exogenously-induced hypercortisolemia on autonomic dysfunction under the systemic inflammatory manifestations mediated by low-dose endotoxin. Solid lines simulate the progression of a self-limited endotoxin-induced inflammatory response while dashed lines reflect the antecedent period of exogenouslyinduced hypercortisolemia which is initiated $6 \mathrm{~h}$ prior to LPS administration and continued for $6 \mathrm{~h}$ after endotoxin $\left(\mathrm{w}_{\mathrm{Fex}}=1\right)$. Solid markers and open circles refer to experimental data (expressed as mean \pm SEM) under conditions of acute endotoxin injury and prior hydrocortisone infusion respectively which do not vary across the two experimental conditions (LPS, Cort-6h+LPS). Such prior cortisol infusion modulates cytokine responses $(\mathrm{P}, \mathrm{A})$ and hormonal responses (EPI) but there is no change in overall system's adaptability as assessed by HRV (solid and dashed HRV lines overlap)........ 162

Figure 6.8: Dose-dependent modulation in the progression of the inflammatory reaction due to short-term epinephrine infusion $\left(\mathrm{W}_{\mathrm{EPI}, \mathrm{ex}}=1\right)$, initiated $3 \mathrm{~h}$ before LPS and continued for $6 \mathrm{~h}$ after LPS at increasing values of the parameter $\mathrm{R}_{\mathrm{in}, \mathrm{EPI}}=5,10,20$. Such intervention potentiates, in a dose dependent manner (dashed and dotted lines), the secretion of epinephrine from SNS that through cAMP anti-inflammatory signaling can protect, in part, the host response attenuating the pro-inflammatory response (P). Such attenuation in the pro-inflammatory response relative to endotoxin administration (solid xxii 
lines) does not extend to changes in autonomic balance (HRV) as represented by the superimposition of predicted HRV dynamics (solid and dashed lines overlap). Solid markers and open circles refer to relevant experimental data (expressed as mean $\pm \mathrm{SEM}$ ) under conditions of low-dose endotoxin administration and prior epinephrine infusion respectively. These data have not been used as a training dataset but rather to validate the structure of the proposed model. Descriptive statistics in the original analysis (Jan, Coyle et al. 2009) show that there was no significant variation between these experimental measurements (solid markers vs. open circles) from $0 \mathrm{hr}$ until 24 hours after LPS exposure. 164

Figure 6.9: Schematic illustration of the Warner model representing the relationship between stimulation of efferent sympathetic nerve activity to the heart and heart rate (HR). $A_{1}$ represents the concentration of sympathetic neurotransmitter (catecholamine) at the nerve ending and $f_{1}$ represents the frequency stimulates preformed on the nerve; $A_{o}$ represents the concentration of catecholamine in peripheral tissues (i.e. blood); $\mathrm{A}_{2}$ represents the concentration at the active site on sinoatrial (SA) node which must react with chemical substance B to produce a change in heart rate, adapted from (Warner and Cox 1962) 170

Figure 6.10: Network topology of the multiscale model of human endotoxemia for the assessment of autonomic heart rate regulation. Elementary pro-inflammatory pathways (i.e. NF-kB signaling module) triggered by the recognition process of endotoxin (LPS) from its signaling receptor (TLR4, R) propagate the effect of LPS signaling on the transcriptional (cellular) response level (P, A, E). Essential modules associated with the release of stress hormones (cortisol (F), epinephrine (EPI)) from neuroendocrine axis xxiii 
(HPA, SNS) coupled with their anti-inflammatory influence on the host are further considered. Finally, at the systemic level, biochemical reactions associated with the release, binding and degradation of cardiac (sympathetic) neurotransmitters $\left(\mathrm{A}_{1}, \mathrm{~A}_{2}\right)$ on the SA node are also incorporated. Efferent autonomic outflow is represented by sympathetic $\left(\mathrm{T}_{\text {sym }}\right)$ and parasympathetic activities $\left(\mathrm{T}_{\text {par }}\right)$ that act antagonistically giving rise to changes in heart rate (HR) response assessed by clinical monitoring of vital signs.

Figure 6.11: Dynamic profiles of the elements that constitute the autonomic heart rate regulation signaling module in human endotoxemia.(A) Simulated concentrations of cardiac (SNS) neurotransmitters at the level of sympathetic nerve ending $\left(\mathrm{A}_{1}\right)$ and at the active site of sinus node (SA) of the heart $\left(\mathrm{A}_{2}\right)$; (B) Efferent sympathetic activity during the progression of the acute inflammatory reaction; (C) Simulated efferent parasympathetic (vagal) activity and (D) Heart rate (HR) response to endotoxin induced inflammation. Human experimental data (open circles) associated with vagal measurements (time domain HRV measure, pNN50) and vital signs (heart rate measurements) are used to calibrate the model. Solid lines (-) represent model predictions under conditions of low-dose endotoxin while $\circ$ circles refer to experimental data expressed as mean \pm SEM. The initial condition of the inflammatory stimulus $(\mathrm{LPS}(\mathrm{t}=0 \mathrm{hr})=1)$ reflects LPS concentration relative to $2-\mathrm{ng} / \mathrm{kg}$ body weight. 180

Figure 6.12: In silico simulation of the cardiovascular (sympathoexcitatory) effects of acute epinephrine infusion on the host initiated $3 \mathrm{hr}$ prior to endotoxin challenge $(\mathrm{t}=0 \mathrm{hr})$ and continued for another $6 \mathrm{hr}$ after LPS. Solid lines simulate the host dynamics under conditions of low-dose endotoxin (LPS) while dashed and dotted lines reflect the xxiv 
dynamics of the host pre-exposed to epinephrine infusion at various doses. The acute preexposure of the host into epinephrine $\left(\mathrm{W}_{\mathrm{EPI}, \mathrm{ex}}=1\right)$ at increasing values of the parameter $\mathrm{R}_{\mathrm{in}, \mathrm{EPI}}=6,12,24$ (represented by $\mathrm{a}, \mathrm{b}$ and $\mathrm{c}$ lines respectively) potentiates (A) circulating levels of epinephrine and (B) the overall efferent sympathetic outflow $\left(\mathrm{T}_{\text {sym }}\right)$ relative to the responses induced by endotoxin administration while (C) vagal activity is significantly attenuated compared to the effect induced only by LPS and finally (D) heart rate response to endotoxin is further increased due to prior epinephrine infusion 182

Figure 6.13: In silico assessment of the cardiovascular implications associated with acute epinephrine infusion on the host. Human experimental data depicted by squares and diamonds represent mean \pm SEM refer to human subjects that received either low dose (2ng/kg BW) LPS or an infusion of epinephrine for $3 \mathrm{hr}$ before LPS administration and continued until +6 hours, respectively. These data are specifically used to validate qualitatively the structure of the proposed human inflammation model employed from (Jan, Coyle et al. 2009) and not to train the model. Descriptive statistics in the original experimental study show that there was a significant change in the parasympathetic activity ( $T_{\text {par }}$ ) and heart rate response (HR) across the two experimental conditions ( $\square$ vs. ৩) from 0hr until 24 hours after LPS exposure. Computationally such situation is captured by the differential predicted responses between dashed and solid lines. We specifically observe that there exists a simulated trajectory of exogenously induced catecholamine excess (represented by dashed lines, $\mathrm{R}_{\mathrm{in}, \mathrm{EPI}}=6$ ) that lies in general agreement with the relevant human experimental data. 184

Figure 6.14: Simulated dose dependent effects of LPS on neuroendocrine immune system interactions. A high concentration of LPS can cause a dysregulation in the host XXV 
dynamics characterized by abnormal transcriptional and hormonal responses. Temporal responses of critical inflammatory components for various initial conditions of the inflammatory stimulus include (A) pro-inflammatory response (P); (B) anti-inflammatory response (A) and stress hormones such as (C) cortisol (F) and (D) epinephrine (EPI). Solid lines simulate the progression of a self-limited inflammatory response at increasing LPS doses $(\operatorname{LPS}(\mathrm{t}=0 \mathrm{hr})=0.4 \mathrm{ng} / \mathrm{kg}, \operatorname{LPS}(\mathrm{t}=0 \mathrm{hr})=4 \mathrm{ng} / \mathrm{kg}$ and $\operatorname{LPS}(\mathrm{t}=0 \mathrm{hr})=6 \mathrm{ng} / \mathrm{kg})$ that are less than the critical threshold (LPS $(\mathrm{t}=0 \mathrm{hr})=8 \mathrm{ng} / \mathrm{kg}$ - four times greater than the nominal value (2ng/kg BW) used to calibrate the model) that gives rise to unresolved inflammatory responses represented by sustained inflammatory markers and hormonal

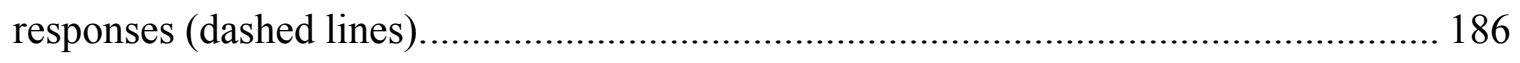

Figure 6.15: Simulated dose dependence of LPS effects on systemic (heart) level responses. A high inflammatory challenge disrupts the autonomic control systems which give rise to prolonged elevations in heart rate. Dynamic responses of (A) catecholamines at the sinus node of the heart $\left(\mathrm{A}_{2}\right)$; (B) efferent sympathetic, (C) parasympathetic activities and (D) heart rate (HR) responses to increasing levels of the inflammatory stimulus (LPS). While solid lines represent constrained inflammatory responses, dashed lines refer to a persistent inflammatory response simulated by high LPS concentration given that such situation can be equated to the severely stressed clinical phenotype manifested as sustained elevations in heart rate (cardiovascular instability). 187

Figure 6.16: Dynamic inflammatory cellular and physiological responses as a function of time after the administration of high inflammatory challenge (dashed lines) given that at time point $0(t=0 \mathrm{hr})$ solid lines represent "virtual" human subjects that receive an infusion of epinephrine. The acute pre-exposure of the host into epinephrine (initiated $3 \mathrm{hr}$ xxvi 
before LPS and continued until $+6 \mathrm{hr}$ after endotoxin, i.e. $\mathrm{R}_{\mathrm{in}, \mathrm{EPI}}=6$ ) attenuates the proinflammatory response $(\mathrm{P})$, relative to the effect mediated by high concentration of LPS, via potentiation of the anti-inflammatory component of the host (A). In addition to the anti-inflammatory role of epinephrine, exogenous up-regulation in circulating levels of epinephrine increase the efferent sympathetic activity $\left(\mathrm{T}_{\text {sym }}\right)$ which is followed by further reduction in vagal function $\left(\mathrm{T}_{\mathrm{par}}\right)$ and these autonomic changes mediate early tachycardia (HR) which is eventually restored within 24 hours.

Figure 6.17: Modulation of the progression of unresolved inflammatory response due to high LPS concentration under conditions of hydrocortisone infusion. The effect of lowdose steroid administration initiated $6 \mathrm{hr}$ before high LPS concentration and continued until $+6 \mathrm{hr}$ after LPS is simulated in solid lines. Such exogenously-induced hypercortisolemia $\left(\mathrm{w}_{\mathrm{Fex}}=1, \mathrm{R}_{\mathrm{in}, \mathrm{F}}=2.922-\right.$ parameters taken from our prior work (Foteinou, Calvano et al. 2010-in press) potentiates total plasma concentration of cortisol a shown in panel (A) and modulates cytokine and hormonal responses. Circulating levels of epinephrine are attenuated in response to antecedent periods of hypercortisolemia relative to the excessive adrenergic response which is illustrated in panel (B). At the autonomic level such attenuation is expected to reduce efferent sympathetic activity mediating $(C)$ an increase in implied vagal function $\left(\mathrm{T}_{\mathrm{par}}\right)$ and finally $(\mathrm{D})$ controlling heart rate as shown by reversibility in the progression of the inflammatory reaction towards homeostasis (baseline) - "recovery phase" (solid lines) 


\section{Chapter 1}

\section{The Problem of Systemic Inflammation}

\subsection{Clinical relevance}

More than 40 million major surgical operations are performed annually in the United States, of which as many as two million are complicated by surgical site infections (Vogel, Dombrovskiy et al. 2009). Surgical adverse effects contribute significantly to postoperative morbidity by perturbing the immune system towards a severely suppressed state that promotes sepsis (Bruce, Russell et al. 2001). Surgical patients account for approximately $30 \%$ of all sepsis patients (Angus, Linde-Zwirble et al. 2001) and the present incidence of acquired surgical site infections is likely to continue to increase among nontrauma surgical patients (Lowry 2009). Sepsis is a syndrome resulting from massive, acute activation of the systemic inflammatory response (SIRS) in the setting of severe infection (Angus, Linde-Zwirble et al. 2001; Decker 2004). Severe sepsis has a substantial impact on healthcare resources and expenditure with an annual incidence exceeding three quarters of a million cases, over $25 \%$ overall in-hospital mortality rate and an average cost per case of more than US\$20,000 (Angus, Linde-Zwirble et al. 2001). Such a mortality rate is translated to over 200,000 deaths annually nationwide and therefore sepsis remains to be the $10^{\text {th }}$ leading cause of death in United States (Kochanek and Smith 2004).

The manifestation of SIRS criteria is the common clinical phenotype of stressed surgical patients and reflects the presence of consequential systemic inflammation 
(Lowry 2009). The clinical signs of a systemic inflammatory response syndrome are related to: (i) a body temperature greater than $38^{\circ} \mathrm{C}$ (hyperthermia) or less than $36^{\circ} \mathrm{C}$ (hypothermia), (ii) an increased heart rate, (ii) tachypnea, (iii) hyperventilation and hypotension as well as (iii) an alteration in white blood cell count (Bone, Balk et al. 1992). However, systemic inflammation is not inherently detrimental. Inflammatory processes are required for immune surveillance and regeneration after injury during which multiple cell types are deployed to locate pathogens, recruit cells and eventually eliminate the offenders and restore homeostasis (Cavaillon and Annane 2006). When anti-inflammatory processes fail, inflammation becomes prolonged and can lead to uncontrolled systemic inflammation which, in turn, can eventuate in various disease conditions or aggravate an already existing disease process (Krishnamoorthy and Honn 2006). It is, therefore, a dysregulation of the resolution of inflammation that, in many cases, causes detrimental effects for the host.

\subsection{Neuro-immune crosstalk}

Physiological mechanisms regulating the inflammatory response involve not only the local release of anti-inflammatory cytokines but also hormonal influences (Czura and Tracey 2005). Recent studies indicate that the central nervous system (CNS) is a pivotal regulator of the immune response (Blalock 2002). A primary stress response pathway by which the CNS regulates the immune system is the hypothalamic-pituitary adrenal axis (HPA), through the production of glucocorticoids and other immunomodulatory signals. Further, activation of the sympathetic division (SNS) of the autonomic nervous system regulates immune function primarily via the release of adrenergic neurotransmitters (Elenkov, Wilder et al. 2000). Although most research has focused on the sympathetic 
immunomodulatory output, it recently became clear that the other arm of CNS, the parasympathetic division (PNS) is also involved in the reflex regulation of inflammation (Pavlov and Tracey 2004). These functions are integrated through a network of complex interactions between the immune, neuro-endocrine and autonomic systems, Figure 1.1. The integrity of this circuitry is essential for maintaining physiological homeostasis and therefore disruption of these functions may have untoward effects (Sharshar, Hopkinson et al. 2005).

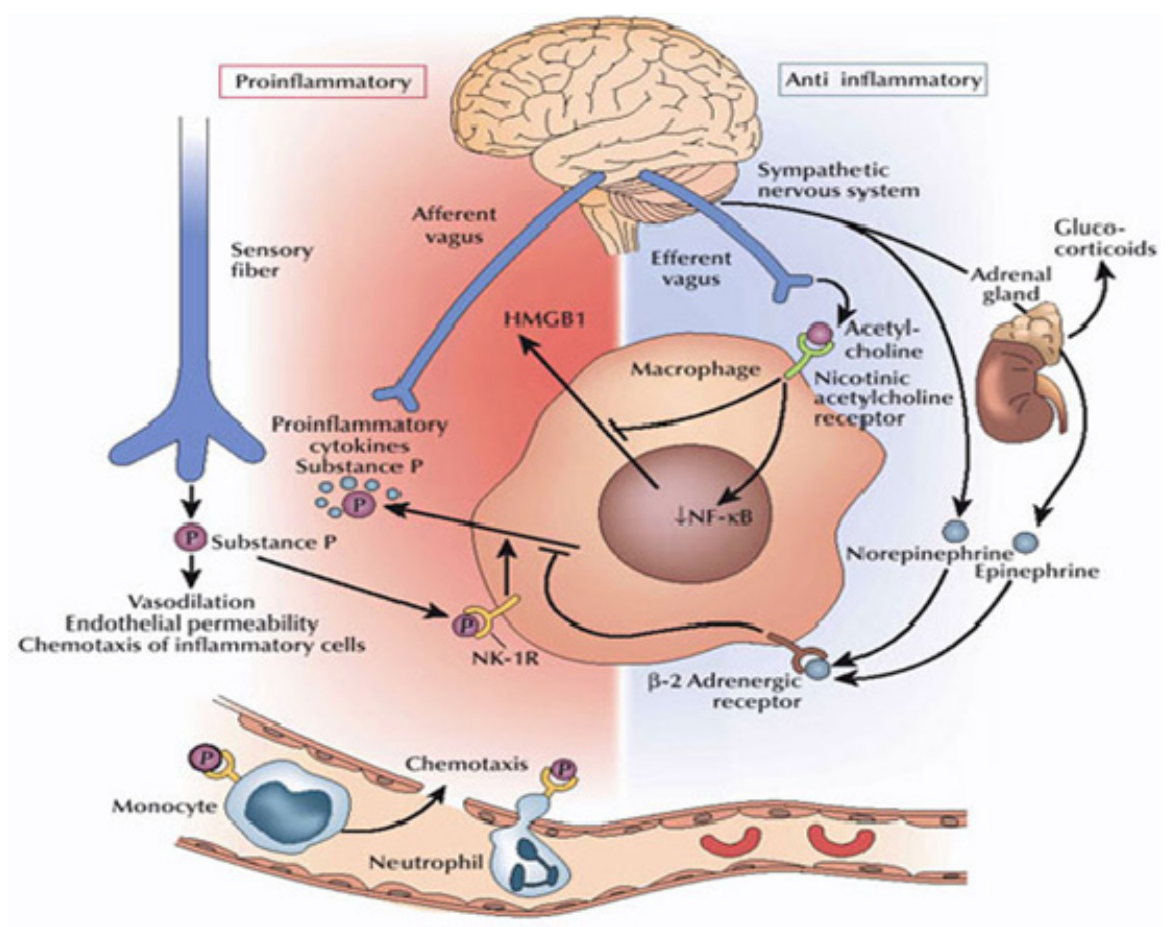

Figure 1.1: Hormonal and neural regulation of immunity. The neuro-immune crosstalk is comprised of a descending pathway linking the CNS with peripheral immune tissues and a parallel afferent arm that links the immune system with the CNS. The CNS is a pivotal regulator of the immune response and controls inflammation at multiple levels including activation of the neuroendocrine axis (epinephrine, glucocorticoids) and the vagus nerve (acetylcholine). Adapted from (Matthay and Ware 2004). 


\subsection{Decomplexification and critical illness}

A characteristic feature of deterioration in the physiologic status of the host is the evolved state of diminished signal variability among organ systems including the innate immune responsiveness and central nervous systems (Lowry and Calvano 2008). Clinical measures of heart rate variability (HRV) are non-invasive assessments that may reflect real-time alterations of physiologic status (Norris, Ozdas et al. 2006). As a potential surrogate marker for systemic decomplexification, diminished HRV has received increasing attention in critical illness (Winchell and Hoyt 1996). Decreases in HRV, i.e., increases in regularity, have also been extensively studied and characterized as generalized responses to human endotoxemia (Godin, Fleisher et al. 1996; Rassias, Holzberger et al. 2005). It has been hypothesized that the reduction in HRV represents an increased isolation of the heart from other organs (Seely and Christou 2000). This hypothesis, originally introduced by Godin and Buchman (Godin and Buchman 1996), suggests that healthy organs behave like biological oscillators coupled to one another. Thus, reduced HRV reflects systemic-level loss of high level signal variability and is associated with a less "healthy" state not only in patients with cardiovascular diseases but also in other critically ill conditions that involve injury, severe infection and sepsis (Borovikova, Ivanova et al. 2000; Morris, Norris et al. 2006; Morris, Norris et al. 2007; Norris, Morris et al. 2005; Norris, Ozdas et al. 2006)

\subsection{The Pathophysiology of systemic inflammation}

An overwhelming systemic inflammatory response (SIRS) can compromise the function of distinct organs leading to multiple organ dysfunction syndrome (MODS) (Matsuda and Hattori 2006). Local infection develops into a systemic inflammatory response syndrome 
(SIRS) that is characterized by the stimulation of various inflammatory mediators which are under the tight control of sepsis-related inhibitors that embrace the compensatory anti-inflammatory response syndrome (CARS), Figure 1.2.

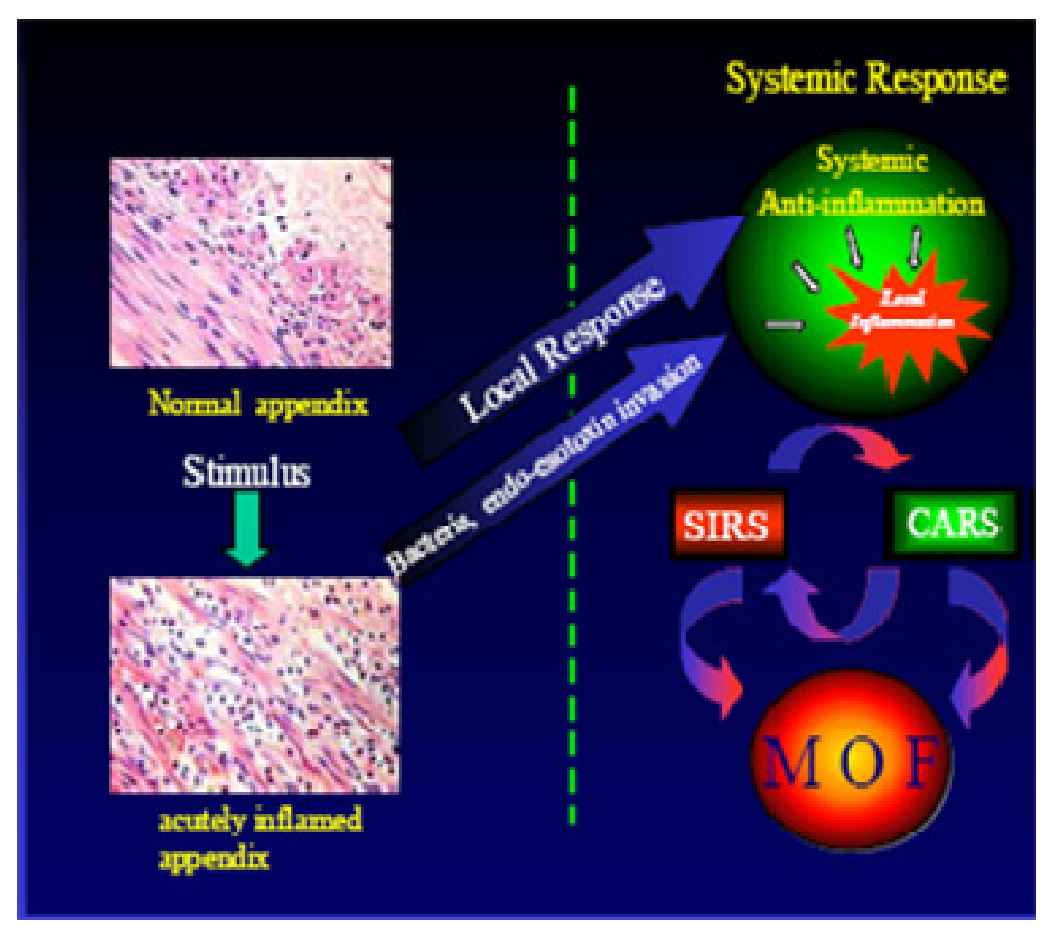

Figure 1.2: The complex mechanisms of the host defense system. The initial pro-inflammatory response may be uncontrolled causing the systemic inflammatory response syndrome (SIRS). A compensatory antiinflammatory response (CARS) may also be triggered and an imbalance between SIRS and CARS cal lead to multiple organ failure (MOF) (Tetta, Fonsato et al. 2005).

During the progression of severe inflammation the homeostatic balance between the two essential arms of the innate immune system (SIRS and CARS) is disrupted being the primary cause of multiple organ failure (MOF) (Vincent 2006). An imbalance between SIRS and CARS accounts for an increase in the circulation of mediators that have significant impact on systemic effects including endothelial damage, fibrinolytic, complement activities and hemodynamic shock followed by vasoparalysis. The dynamics of inflammation are highly complex and in particular when a pathogen crosses the host's 
defense barriers, the innate immune system is activated. It consists of both soluble and cellular elements (macrophages, neutrophils, monocytes, dendritic cells and natural killer cells) (Hotchkiss and Karl 2003). The "chemo-attraction" of such immune cells stimulates the production of pro-inflammatory mediators (TNF-a, IL1) which serve as inter-cellular signal for the production of anti-inflammatory mediators (IL-10, TGF- $\beta$ ).

In physiological conditions there is a homeostatic control between the SIRSassociated mediators and those that are associated with the compensatory antiinflammatory response syndrome. In addition to this, the inflammatory process results in the release of free radicals that mediates tissue destruction killing bacteria. and an imbalance in their regulatory activity may contribute significantly to the inflammatory process (Winrow, Winyard et al. 1993). Another characteristic of septic patients are abnormalities in coagulation cascade which results in hypotension and hypoperfusion of major organs that contribute to death (Bochud and Calandra 2003).

It was initially believed that an aberrant production of pro-inflammatory mediators is responsible for the pathogenesis of sepsis (Martich, Danner et al. 1991; Michie, Manogue et al. 1988; Suffredini, Fromm et al. 1989). However, other studies (Brandtzaeg, Osnes et al. 1996; Munoz, Carlet et al. 1991; Randow, Syrbe et al. 1995) address the issue of septic hyporesponsiveness as reduced capacity of immune cells to produce several cytokines associated with the persistent activation of potent inhibitors. On the other hand, there is evidence (Bone, Grodzin et al. 1997) that in patients with either infectious or non-infectious sepsis, SIRS and CARS may co-exist and therefore there is the imbalance between SIRS and CARS that can lead to an exuberant inflammatory response. Thus, although there is active research area on unraveling the ensuing physiological alterations 
that characterize sepsis, significant challenges remain to be solved before this devastating process is understood (Vincent and Abraham 2006). Notably, we need to identify new ways of studying the behavior of complex biological systems.

\subsection{The era of interdisciplinary and translational research}

The term "translational research" has been coined by the American Physiological Society (APS) (Hall 2001) as the "transfer of knowledge gained from basic research to new and improved methods of preventing, diagnosing or treating disease as well as the transfer of clinical insights into hypotheses that can be tested and validated in the research lab". The definition implies that the process is bidirectional from the research track to the clinical one as well as from the clinical research back to the research track. Apparently, the gap between the basic research and clinical track (the two arms of the translational research) accounts for the failure of various potential therapies to treat human disease. Recognizing such a growing gap the APS has taken several steps to encourage translational research (Hall 2001). Such an effort is being appreciated and continued by many organizations, including the National Institutes of Health (NIH), American Cancer Society e.t.c.(Gelijns, Rosenberg et al. 1998).

In order to promote a systematic integration across multiple disciplines to help bridge this gap in the context of inflammation following traumatic injury the NIH recently funded the development of a large-scale collaborative glue grant research program known as Inflammation and the Host Response to Injury (Klein, Silver et al. 2006). Participating institutions include hospitals involved in clinical research studies, academic medical centers that perform analytical studies on blood and tissue samples, and informatics and statistics centers that develop databases and analyze data. Furthermore, the current 
definition of NIH's Road Map to Medical Research ${ }^{1}$ clearly identifies and states the importance of a deeper and better understanding of inflammation since it is broadly implicated in many diseases and conditions. Subsequently, this collaborative research program encourages investigators from different research areas to work together and indentify the missing pieces of complex biomedical research problems (systemic inflammation). Remarkably, such a program bridges the gap between basic and clinical research given the fact that it encompasses funded programs that are associated with the design of high-throughput experiments (genomics, proteomics, metabolomics) coupled with the patient-oriented research. The multi-disciplinary character of this program allows researchers to generate and share various quantitative data raising the question of what is an appropriate analysis that offers the possibility to unravel the salient features of a complex phenomenon.

Integrated initiatives are identified as valuable in uncovering as-yet-unknown immune mechanisms and mediators of inflammation as well as genetic factors, environmental triggers, and the relationship of inflammation to disease. Currently, the accumulation of massive amounts of data necessitates the development of computational approaches that will shed useful insight on the outcome of a patient. Along this critical path the FDA stresses the need to incorporate advanced computational methods encouraging the development of disease models in that they can improve our understanding about the behavior of the system predicting the human response and indentifying surrogate endpoints that trace the trajectory of the disease facilitating the design of drugs and devices (Miller, Ewy et al. 2005).

\footnotetext{
${ }^{1}$ http://nihroadmap.nih.gov/2008initiatives.asp
} 


\subsection{Rationale for mathematical modeling}

The progress of high throughput technologies associated with large-scale gene, protein and metabolite measurements have dramatically accelerate the hypothesis-driven development of in silico disease models. Mathematical modeling offers the opportunity of studying the dynamics of the interacting elements of a complex system while it provides a systematic framework for integrating research work from many disciplines (Sontag 2004). Systems-based modeling enables the systematic integration of massive amounts of relevant information shedding invaluable insight into the progression of the disease state and into possible therapeutic interventions (Rajasethupathy, Vayttaden et al. 2005). Oftentimes, in silico models are viewed as the "digital analogs of transgenic animals" in which the activity of the immune system can be manipulated in controlled conditions (Kitano 2002).

Mathematical models integrating the interacting elements of the unified inflammatory response offer the opportunity to establish a causal inference relationship through the manipulation of the corresponding dynamic elements (Lauffenburger and Kennedy 1980). Systems-based translational research considers physiological conditions as dynamically evolving "systems" with clearly identified boundaries and rules defining their response (An, Hunt et al. 2007; Seely and Christou 2000). As a result, there is a growing research effort towards the development of systems-based, quantitative models of the inflammatory response of various degrees of complexity driven by the premise that such models can potentially enable the translation of knowledge from bench to bedside (Chung, Laramie et al. 2006). 


\subsection{Motivation and outline of the thesis}

The systemic inflammatory response syndrome often accompanies critical illnesses and can be an important cause of morbidity and mortality. Although considerable progress has been made in elucidating many of the components of inflammation and their regulation, most hypotheses related to the management and treatment of severe human inflammation have failed rigorous clinical testing. A key reason for this conundrum is the difficulty of predicting the impact of manipulating individual components of the highly complex and non-linear inflammatory response making model based approaches appealing. Therefore, the intricacies in translating basic research to clinical practice is recognized as a challenge that needs to be overcome in order to successfully transfer the information from the pre-clinical to the clinical stage.

Significant opportunities emerge in the context of systems biology which aims at the deconvolution of complex phenomena to their constituent elements and the quantification of the dynamic interactions between these components through the development of appropriate computational and mathematical models. Systems biology is based on the premise that biological phenotypes can be described as the emergent response of a host to an external signal or perturbations. The development of in silico models has been accelerated by the availability of massive amounts of data related to dynamic cellular and molecular-level responses providing the underlying molecular signatures that drive macroscopic phenotypic observations. As a result, there is a growing research effort towards the development of systems-based, quantitative models of the inflammatory response and their potential role in systems-based translational research is discussed in Chapter 2. 
However, a number of critical issues need to be addressed and are outlined in Chapter 3. In order to characterize a dynamic system we need to have the ability to properly describe the state space defining the response. In doing so, we developed a systematic computational framework that decomposes high-dimensional microarray data into an elementary set of comprehensive responses; characterized as the "blueprints" of the orchestrated dynamics of the perturbed biological system. Such responses effectively decompose the overall dynamics and present the constitutive elements of the integrated systemic response. We need, therefore, to develop appropriate computational methodologies that will allow the identification of these basic response elements and are presented in Chapter 4.

Once the state space has been identified we need to develop the appropriate wiring architectures that convolute a multitude of external signals. Although black-box modeling has found widespread applications in systems biology, we wish to explore the possibility of developing more mechanistic-based and simplified representations of the dynamics. By incorporating biological information in the form of critical signaling cascades and kinetic rules we would probably be able to develop more interpretable and physiologically relevant computational models. As such, cellular physicochemical host response models are proposed in Chapter 5 to assess the propagation of an external perturbation as the emergence response of a network of interacting components essential for the onset, resolution and control of the host response.

Further, in an effort to develop in silico methodologies that will enable translational research to elucidate mechanisms by which macroscopic responses, at the physiome level, emerge as the result of propagating information, in the form of disturbances, across 
an intricate web of interacting modules, functional links between cellular events and physiological responses need to be established. A vital enabler in that respect is the development of systems-level analysis that integrates data across multiple scales and models essential features of the multiscale nature of the response. Specifically, multiscale human inflammation models are developed in Chapter 6 that quantify critical aspects of the complex bidirectional communication between the CNS and the immune response and assess cardiovascular (systemic) abnormalities by incorporating explicitly phenotypic expressions such as clinical heart rate measurements that correlate with the severity of injury (illness). Thus, the goal of this research is to demonstrate the feasibility of a multiscale model of human endotoxemia as a prototype model of acute inflammation in humans that couples essential regulatory processes across the host from the cellular to the systemic level.

Finally, we wish to demonstrate not only the potential implications of the proposed integrative approach towards the study of the inflammatory response, but also to advocate the possibility of the generalization of this framework in a wide range of disease progression models and thus enabling the use of systems biology in translational research. It is therefore the goal of this dissertation to demonstrate the feasibility of the proposed approach as a notional template for multiscale modeling in human physiology. 


\section{Chapter 2}

\section{Background}

Bacterial infection, trauma, surgery and biological stressors in general, induce an acute inflammatory response, characterized by a cascade of events that involve initiation of the reaction, progression, and termination followed by resolution of inflammation. Under normal circumstances, this inflammatory response is self-limited, and once the pathogens are cleared, reparative processes begin and the response then abates (Laroux 2004). Oftentimes pro-inflammatory responses prevail or anti-inflammatory processes fail, and an amplified runaway inflammation turns what is normally a beneficial reparative process into a detrimental physiological state characterized by an inflammatory state of the entire system (Nystrom 1998).

Despite the growing understanding of the cellular and molecular mechanisms of systemic inflammatory response syndrome (Tetta, Fonsato et al. 2005) and the success of pre-clinical studies, not many effective therapies exist and few drugs are known to reduce mortality in clinical trials (Annane, Sebille et al. 2002; Annane, Bellissant et al. 2004a, b; Arzt, Sauer et al. 1994; Bernard, Vincent et al. 2001; Kerschen, Fernandez et al. 2007; Klaitman and Almog 2003; Marshall 2003; Meduri, Headley et al. 1998; Riedemann, Guo et al. 2003). Even the improved capacity to acquire quantitative data in a clinical setting has generally failed to improve outcomes in acutely ill patients. Thus, the intricacies in translating basic research to clinical practice are recognized as a challenge impeding the successful transfer of information from the pre-clinical to the clinical stage (Marshall 2005; Marshall, Deitch et al. 2005). This challenge is often linked to the 
growing gap between basic and clinical research (Hall 2001) and there is growing interest to bridge the two through translational research. As such, the nature of the response has led researchers to the realization that mathematical models of inflammation might provide rational leads for the development of strategies that promote the resolution of the response and the eventual establishment of homeostasis (Seely and Christou 2000). Such models are critical enablers in advancing the translational potential of clinical research, a subject recently reviewed (Foteinou, Calvano et al. 2009c).

\subsection{In-silico modeling of inflammation}

The modeling approaches fall broadly in two categories: those based on explicit dynamics (Day, Rubin et al. 2006; Reynolds, Rubin et al. 2006) and agent based models which are discrete in time and space (An 2004). One of the earliest mathematical models of inflammation dates back to the early 1980s when Lauffenburger and co-workers (Lauffenburger and Kennedy 1980) described the local tissue inflammatory response to bacterial invasion. In this model the leukocytes are continuously distributed whilst their accumulation and efficiency in localization within the inflammatory lesion coupled with their phagocytic activity determine the resolution of infection. This model expresses the dynamic interaction between the invader and a homogeneous leukocyte population using a two variable model that consists of bacterial and leukocyte densities. An extension of this model replaced the single cell-target with a density number associated with the target population (Fisher and Lauffenburger 1990). In this model the principal goal is to address the effect of factors such as chemotaxis, cell speed and persistence on target elimination dynamics. Further attempts (Mayer, Zaenker et al. 1995) explored the interaction of the immune system with a target population (bacteria, viruses). Such analyses explore non- 
linear interaction rules between the immune and target cells that determine the outcome of the immune response. Alternative modeling approaches placed emphasis on simulating interactions at the cellular level in response to an infection (Detilleux, Vangroenweghe et al. 2006).

Among the simplest, yet very informative, inflammation models incorporating measured quantities is the one proposed by Kumar et al. (Kumar, Clermont et al. 2004). The model tracks three basic variables indicative of the onset, progress and resolution of the inflammatory response, which include the pathogen, pro- and late- inflammatory mediators. Specifically, the pathogen ( $p$ ) is the instigator of the inflammatory response while a pro-inflammatory mediator $(\mathrm{m})$ captures the combined effect of various inflammatory cells and finally the late pro-inflammatory mediators (1). The underlying basis of this model is to explore the interaction between early and late pro-inflammatory mediators. The interaction between the pathogen $(\mathrm{p})$ and the pro-inflammatory cells $(\mathrm{m})$ is modeled using the principles of a predator-prey model whilst the interactions among the other model elements are characterized by algebraic functions that can simulate an early (m) and a late (1) upregulated dynamic response. This model explores the dynamic interaction of particular aspects of the inflammatory response investigating regimes of parameter values that can account for inflammatory relevant scenarios.

Later it was suggested (Day, Rubin et al. 2006; Reynolds, Rubin et al. 2006) that the outcome of a healthy inflammatory response is determined by a balanced regulation in the dynamics of pro- and anti-inflammation. In this model, as seen in Figure 2.1(left), the pathogen $(\mathrm{P})$ is the initiating event that stimulates the activation of pro-inflammatory mediators $\left(\mathrm{N}^{*}\right)$ that can be either cytokines or immune cells (macrophages). 


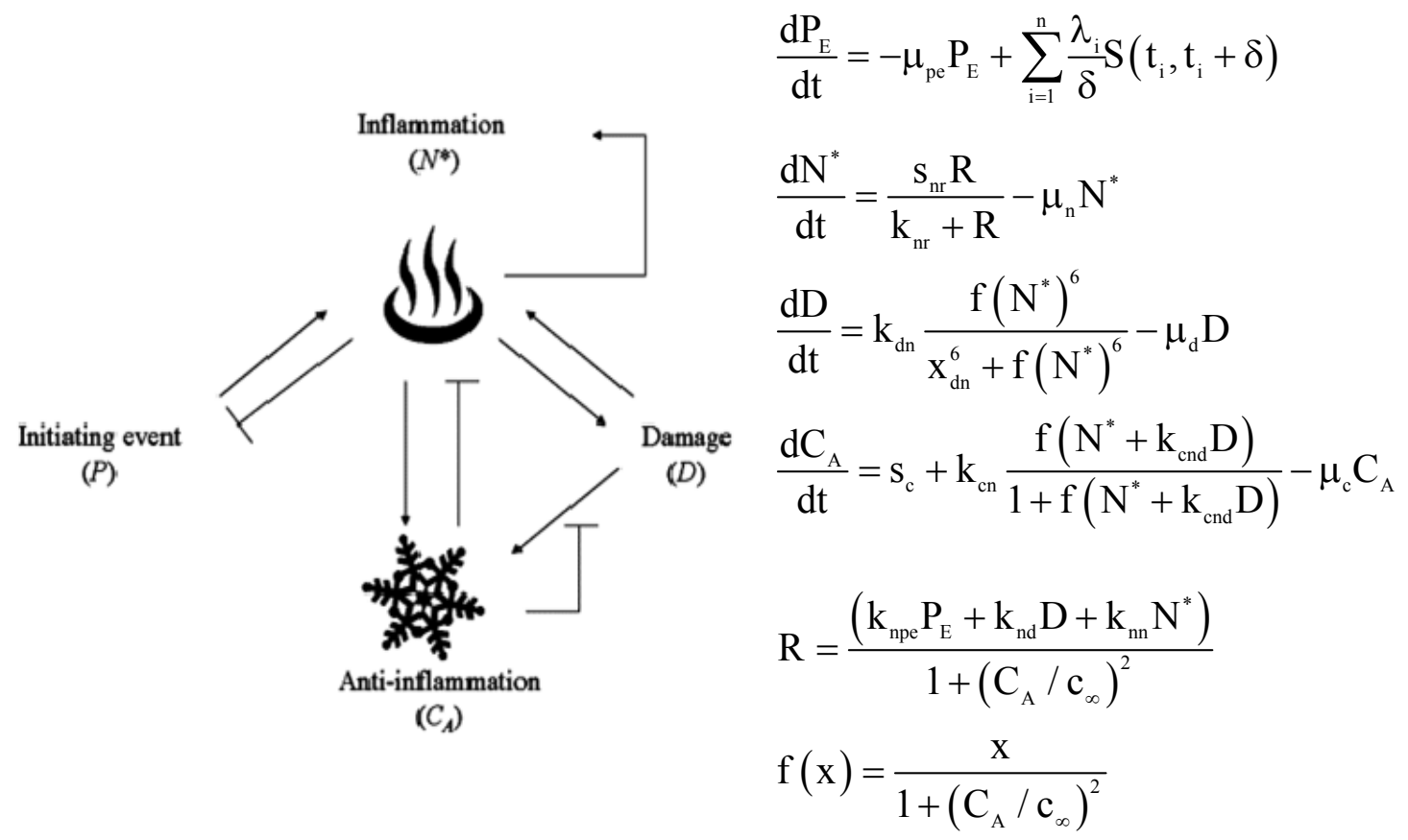

Figure 2.1: (left) Qualitative structure of the four variable model of acute inflammation. Infection (P) triggers inflammation $\left(\mathrm{N}^{*}\right)$ while regulatory mechanisms conceptualized as anti-inflammation $\left(\mathrm{C}_{\mathrm{A}}\right)$ are triggered almost simultaneously. Excessive inflammation results in tissue dysfunction (D); (right) Model dynamics proposed and developed in (Reynolds, Rubin et al. 2006). 
Once the pro-inflammatory response is activated, it serves as a subsequent signal for stimulating the anti-inflammatory component of inflammation. Moreover, the increased expression of pro-inflammatory mediators can cause tissue damage and is expressed via the variable (D). This variable serves as a marker for tissue damage/organ dysfunction (unmeasurable variable) whilst $\mathrm{C}_{\mathrm{A}}$ is considered to be the regulatory component (antiinflammation) of the system. The dynamic interactions of this reduced set of inflammatory components are modeled mathematically using special functional forms as seen in Figure 2.1(right).

In a further refinement of this model (Clermont, Bartels et al. 2004) the dynamic evolution of effector cells (macrophages, neutrophils) is distinguished from the corresponding activation of effector cytokines and there is emphasis on the importance of modeling crucial signaling pathways (i.e. complement activation). In particular, the dynamic state of the model depends on the state of various inflammatory components that are related to: (1) pathogen $(\mathrm{P})$, (2) pathogen-derived endotoxin $\left(\mathrm{P}_{\mathrm{E}}\right)$, (3) resting macrophages (MR), (4) activated macrophages (MA), (5) activated neutrophils (NA), (6) nitric oxide synthase activity (NOD), (7) circulating levels of nitric oxide (NO), (8) tumor necrosis factor (TNF), (9) interleukin -6 (IL6), (10) generic anti-inflammatory activity (CA, CAI, CAR), (10) activated protein C (PC), (11) tissue factor (TF), (12) thrombin (TH), (13) blood pressure (B) and (14) tissue damage (D). All the interacting elements are quantified and illustrated in Figure 2.2. 


$$
\begin{aligned}
& \frac{\mathrm{dP}}{\mathrm{dt}}=\mathrm{k}_{\mathrm{pg}} \cdot \mathrm{P} \cdot\left(1-\mathrm{k}_{\mathrm{ps}} \cdot \mathrm{P}\right) \cdot \operatorname{heav}\left(\mathrm{P}-\mathrm{P}_{0}\right)-\left(\mathrm{k}_{\mathrm{pm}} \cdot \mathrm{MA}+\mathrm{k}_{\mathrm{pno}} \cdot \mathrm{NO}+\mathrm{k}_{\mathrm{p} 02} \cdot \mathrm{O} 2+\mathrm{k}_{\mathrm{ab}} \cdot \operatorname{heav}(\mathrm{t}-\mathrm{tab})\right) \cdot \mathrm{P} \\
& \frac{\mathrm{dPE}}{\mathrm{dt}}=\mathrm{k}_{\mathrm{pp}} \cdot \mathrm{P}+\mathrm{k}_{\mathrm{pg}} \cdot \mathrm{P} \cdot\left(1-\mathrm{k}_{\mathrm{ps}} \cdot \mathrm{P}\right) \cdot \operatorname{heav}(\mathrm{P}-\mathrm{P} 0)-\left(\begin{array}{l}
\mathrm{k}_{\mathrm{pm}} \cdot \mathrm{MA}+\mathrm{k}_{\mathrm{pno}} \cdot \mathrm{NO}+\mathrm{k}_{\mathrm{po} 2} \cdot \mathrm{O} 2 \\
+\mathrm{k}_{\mathrm{ab}} \cdot h e a v\left(\mathrm{t}-\mathrm{ta}-\mathrm{k}_{\mathrm{pe}} \cdot \mathrm{PE}\right)
\end{array}\right) \\
& \frac{\mathrm{dMR}}{\mathrm{dt}}=-\left(\mathrm{k}_{\mathrm{mp}} \cdot \mathrm{P}+\mathrm{k}_{\mathrm{mpe}} \cdot \mathrm{PE}+\mathrm{k}_{\mathrm{md}} \cdot \mathrm{D}\right) \cdot\left(\begin{array}{l}
\mathrm{S}_{\mathrm{m}}+\mathrm{f} 2\left(\mathrm{TNF}, \mathrm{x}_{\mathrm{tnf}}\right) \cdot \\
\mathrm{k}_{\mathrm{m} 6} \cdot \mathrm{f} 2\left(\mathrm{IL} 6, \mathrm{x}_{\mathrm{i} 16}\right) \cdot \mathrm{fs} 2\left(\mathrm{CA}, \mathrm{x}_{\mathrm{ca}}\right)
\end{array}\right) \cdot \mathrm{MR} \\
& +\mathrm{k}_{\mathrm{mm}} \cdot \mathrm{f}\left(\mathrm{s}_{\text {noa }} \cdot \mathrm{TNF}+\mathrm{s}_{\text {noa }} \cdot \mathrm{PE}+\mathrm{NO}, \mathrm{x}_{\mathrm{t}}\right)-\mathrm{k}_{\mathrm{mr}} \cdot \mathrm{MR}+\mathrm{S}_{\mathrm{m}} \\
& \frac{\mathrm{dMA}}{\mathrm{dt}}=\left(\mathrm{k}_{\mathrm{mp}} \cdot \mathrm{P}+\mathrm{k}_{\mathrm{mpe}} \cdot \mathrm{PE}+\mathrm{k}_{\mathrm{md}} \cdot \mathrm{D}\right) \cdot\left(\begin{array}{l}
\mathrm{S}_{\mathrm{m}}+\mathrm{f} 2\left(\mathrm{TNF}, \mathrm{x}_{\mathrm{tnf}}\right) \cdot \mathrm{k}_{\mathrm{m} 6} \cdot \\
\mathrm{f} 2\left(\mathrm{IL} 6, \mathrm{x}_{\mathrm{il} 6}\right) \cdot \mathrm{fs} 2\left(\mathrm{CA}, \mathrm{x}_{\mathrm{ca}}\right)
\end{array}\right) \cdot \mathrm{MR} \\
& -\mathrm{k}_{\mathrm{ma}} \cdot \mathrm{MA} \\
& \frac{\mathrm{dNA}}{\mathrm{dt}}=\left(\begin{array}{l}
\mathrm{k}_{\mathrm{np}} \cdot \mathrm{f}\left(\mathrm{P}, \mathrm{x}_{\mathrm{t} 2}\right)+\mathrm{k}_{\mathrm{npe}} \cdot \mathrm{f}\left(\mathrm{PE}, \mathrm{x}_{\mathrm{t} 2}\right)+\mathrm{k}_{\mathrm{ntnf}} \cdot \mathrm{f}\left(\mathrm{TNF}, \mathrm{x}_{\mathrm{tnf}}\right)+\mathrm{k}_{\mathrm{n} 6} \cdot \\
\mathrm{f}\left(\mathrm{IL} 6, \mathrm{x}_{\mathrm{i} 16}\right)+\mathrm{k}_{\mathrm{nd}} \cdot \mathrm{f}\left(\mathrm{D}, \mathrm{x}_{\mathrm{t} 2}\right)
\end{array}\right) \cdot \mathrm{NA} \cdot\left(1-\mathrm{k}_{\mathrm{ns}} \cdot \mathrm{NA}\right)- \\
& \left(\left(\mathrm{k}_{\mathrm{mno}} \cdot \mathrm{NO} / \mathrm{s}_{\mathrm{noa}}+\mathrm{k}_{\mathrm{no} 2} \cdot \mathrm{O} 2\right) \cdot \mathrm{NA}-\mathrm{k}_{\mathrm{n}} \cdot\left(\begin{array}{l}
\mathrm{fs}\left(\mathrm{TNF}, \mathrm{x}_{\mathrm{tnf}}\right)+ \\
\mathrm{f}_{\mathrm{s}}\left(\mathrm{IL} 6, \mathrm{x}_{\mathrm{il} 6}\right)
\end{array}\right) \cdot \mathrm{NA}+\mathrm{S}_{\mathrm{n}}\right) \\
& \frac{\mathrm{dNOD}}{\mathrm{dt}}=\left(\mathrm{k}_{\text {non }} \cdot \mathrm{NA}+\mathrm{k}_{\text {nom }} \cdot \mathrm{MA}\right) \cdot \mathrm{fca}\left(\mathrm{CA}, \mathrm{x}_{\mathrm{ca}}\right) \cdot\left(\mathrm{f}\left(\mathrm{TNF}, \mathrm{x}_{\mathrm{tnf}}\right)+\mathrm{f}\left(\mathrm{IL6}, \mathrm{x}_{\mathrm{il} 6}\right)\right)-\mathrm{k}_{\mathrm{nod}} \cdot \mathrm{NOD} \\
& \frac{\mathrm{dNO}}{\mathrm{dt}}=\mathrm{k}_{\mathrm{no}}\left(\mathrm{NOD} \cdot \mathrm{s}_{\mathrm{noa}}-\mathrm{NO}\right) \\
& \frac{\mathrm{dO} 2}{\mathrm{dt}}=\left(\begin{array}{l}
\left(\mathrm{k}_{\mathrm{O} 2 \mathrm{n}} \cdot \mathrm{N}+\mathrm{k}_{\mathrm{O} 2 \mathrm{~m}} \cdot \mathrm{MA}\right) \cdot\left(\mathrm{f}\left(\mathrm{TNF}, \mathrm{x}_{\mathrm{TNF}}\right)+\mathrm{k}_{026} \cdot \mathrm{f}\left(\mathrm{IL} 6, \mathrm{x}_{\mathrm{i} 16}\right)\right)+ \\
\mathrm{k}_{02 \mathrm{n}} \cdot \mathrm{NA} \cdot \mathrm{f}\left(\mathrm{P}, \mathrm{x}_{\mathrm{t}}\right)
\end{array}\right) \cdot \mathrm{fs} 2\left(\mathrm{CA}, \mathrm{x}_{\mathrm{ca}}\right)-\mathrm{k}_{\mathrm{O} 2} \cdot \mathrm{O} 2 \\
& \frac{\mathrm{dTNF}}{\mathrm{dt}}=\left(\mathrm{k}_{\mathrm{tnfn}} \cdot \mathrm{NA}+\mathrm{k}_{\mathrm{tnfmr}} \cdot \mathrm{MR}+\mathrm{k}_{\mathrm{tnfma}} \cdot \mathrm{MA}\right) \cdot \mathrm{fs} 2\left(\mathrm{CA}, \mathrm{x}_{\text {tnfca }}\right) \cdot\left(1+\mathrm{k}_{\mathrm{tnftnf}} \cdot \mathrm{f}\left(\mathrm{TNF}, \mathrm{x}_{\mathrm{tnf}}\right)\right) \\
& -\mathrm{k}_{\mathrm{tnf}} \cdot \mathrm{TNF}-\mathrm{k}_{\mathrm{atnf}} \cdot \text { square }(\text { tiatnf, tiatnf }+ \text { dur }) \cdot \mathrm{TNF} \\
& \frac{\mathrm{dIL6}}{\mathrm{dt}}=\mathrm{k}_{6 \mathrm{~m}} \cdot \mathrm{MA} \cdot\left(1+\mathrm{k}_{6 \mathrm{th}} \cdot \mathrm{f}\left(\mathrm{TH}, \mathrm{x}_{\mathrm{t}}\right) \cdot \mathrm{fs} 2\left(\mathrm{CA}, \mathrm{x}_{\mathrm{t} 2}\right)\right)-\mathrm{k}_{6} \cdot \mathrm{IL6}
\end{aligned}
$$




$$
\begin{aligned}
& \frac{\mathrm{dCAR}}{\mathrm{dt}}=\left(\mathrm{k}_{\mathrm{can}} \cdot \mathrm{N}+\mathrm{k}_{\mathrm{cam}} \cdot \mathrm{MA}\right) \cdot\left(\begin{array}{l}
\mathrm{k}_{\mathrm{catnf}} \cdot \mathrm{f}\left(\mathrm{TNF}, \mathrm{x}_{\mathrm{tnf}}\right)+\mathrm{k}_{\mathrm{ca} 6} \cdot \mathrm{f}\left(\mathrm{IL} 6, \mathrm{x}_{\mathrm{il} 6}\right)+ \\
\mathrm{k}_{\mathrm{cano}} \cdot \mathrm{f}\left(\mathrm{NO}, \mathrm{x}_{\mathrm{t}}\right)+\mathrm{k}_{\mathrm{caO} 2} \cdot \mathrm{f}\left(\mathrm{O} 2, \mathrm{x}_{\mathrm{t}}\right)
\end{array}\right)-\mathrm{k}_{\mathrm{car}} \cdot \mathrm{CAR} \\
& \frac{\mathrm{dCAI}}{\mathrm{dt}}=\mathrm{CAR}-\mathrm{k}_{\mathrm{ca}} \cdot \mathrm{CAI} \\
& \mathrm{CA}=\mathrm{CAI} \cdot \mathrm{k}_{\mathrm{capc}} \cdot \mathrm{PC} \\
& \frac{\mathrm{dTF}}{\mathrm{dt}}=\left(\mathrm{k}_{\mathrm{tfpe}} \cdot \mathrm{PE}+\mathrm{k}_{\mathrm{ttnf}} \cdot \mathrm{TNF}+\mathrm{k}_{\mathrm{tr} 6} \cdot \mathrm{IL} 6\right) \cdot \mathrm{fs}\left(\mathrm{PC}, \mathrm{x}_{\mathrm{t}}\right)-\mathrm{k}_{\mathrm{tf}} \cdot \mathrm{TF} \\
& \frac{\mathrm{dTH}}{\mathrm{dt}}=\left(\mathrm{k}_{\mathrm{th} 1}+\mathrm{k}_{\mathrm{thn}} \cdot \mathrm{TH}\right) \cdot \mathrm{TF}-\mathrm{k}_{\mathrm{th}} \cdot \mathrm{TH} \\
& \frac{\mathrm{dPC}}{\mathrm{dt}}=\mathrm{k}_{\mathrm{pcth}} \cdot \mathrm{TH}-\mathrm{k}_{\mathrm{pc}} \cdot \mathrm{PC} \\
& \frac{\mathrm{dB}}{\mathrm{dt}}=\mathrm{k}_{\mathrm{b}} \cdot\left(\mathrm{B}_{\mathrm{a}}-\mathrm{B}\right)-\left(\left(\mathrm{k}_{\mathrm{bno}} / \mathrm{s}_{\mathrm{noa}}\right) \cdot \mathrm{NO} \cdot \mathrm{fs}\left(\mathrm{O} 2, \mathrm{x}_{\mathrm{t}}\right)+\mathrm{k}_{\mathrm{btnf}} \cdot \mathrm{TNF}+\mathrm{k}_{\mathrm{bth}} \cdot \mathrm{TH}\right) \cdot \mathrm{B} \\
& \frac{\mathrm{dD}}{\mathrm{dt}}=\mathrm{k}_{\mathrm{db}} \cdot\left(1-\left(\mathrm{B} / \mathrm{B}_{\mathrm{a}}\right)\right)+\mathrm{k}_{\mathrm{dtnf}} \cdot \mathrm{TNF}+\mathrm{k}_{\mathrm{dO} 2} \cdot \mathrm{O} 2+\left(\mathrm{k}_{\mathrm{dno}} / \mathrm{s}_{\mathrm{noa}}\right) \cdot \mathrm{NO} \cdot \mathrm{fs}\left(\mathrm{NO}, \mathrm{x}_{\mathrm{t} 2} \cdot \mathrm{s}_{\mathrm{noa}}\right) / \mathrm{s}_{\mathrm{noa}}+ \\
& \mathrm{k}_{\mathrm{dth}} \cdot \mathrm{TH}+\mathrm{k}_{\mathrm{deq}} \cdot \mathrm{O} 2 \cdot \mathrm{e}^{-10 \cdot\left(\mathrm{NO}-\mathrm{s}_{\mathrm{noa}} \cdot 02\right)^{2} / \mathrm{s}_{\mathrm{noa}}^{2}}-\mathrm{k}_{\mathrm{d}} \cdot \mathrm{D}
\end{aligned}
$$

Figure 2.2: A complex, multivariable mathematical model of systemic inflammation (Clermont, Bartels et al. 2004) 
The model as shown in Figure 2.2 is expanded with the inclusion of particular proinflammatory mediators (TNF, IL6) followed by the activation of macrophages and neutrophils (immune cells). As far as the generic anti-inflammatory activity is concerned, this model variable refers to the activity of well-established counter-regulatory components such as IL10, steroids, transforming growth factor- $\beta$ (TGF- $\beta$ ), soluble receptors to pro-inflammatory cytokines that are triggered by pro-inflammatory agents (TNF, IL-6 and NO). In addition to this, the ordinary differential equations that model the tissue factor $(\mathrm{TF})$, thrombin $(\mathrm{TH})$ and the antithrombotic activated protein $\mathrm{C}(\mathrm{PC})$ are associated with the activation of the coagulation and complement pathway. The underlying rationale behind the selection of the aforementioned model elements lies on prior biological knowledge. Extension of this research effort focused towards the development of more generalized inflammatory models accounting for a diverse array of initiating events (Chow, Clermont et al. 2005; Prince, Levy et al. 2006). Models that describe the dynamics of the immune system in response to other infectious agents have also been proposed (Hancioglu, Swigon et al. 2007) characterizing the rates of various processes contributing to the progression of the disease focusing on the control of the infection by the innate and adaptive immunity(Lagoa, Bartels et al. 2006).

An important issue is that of increasing complexity in the model with the lack of a total calibration. Regardless of the fact that there are components in the model that still need calibration, this model offers the opportunity of evaluating immunomodulatory strategies. Thus in (Clermont, Bartels et al. 2004) the authors simulate an anti-TNF clinical trial identifying characteristics of trial populations that are going to be benefited by such an intervention. Such an approach provides us with significant insight into the 
design of clinical trials of immunomodulatory strategies ranging from optimal patient selection to individualized selection of drug dosage ant duration of treatment. Further, in (Chow, Clermont et al. 2005; Prince, Levy et al. 2006) the model analysis revealed that LPS does not mediate cannulation or hemorrhagic shock-induced inflammation via the classic CD14-TLR4 pathway and have also been pivotal in supporting the hypothesis that shock states induced by a wide range of physiological challenges are the emergent result of a universal response despite differences in initiation and modulation. Consequently, in silico models can predict a zone of therapeutic opportunity against inflammation predicting the effect of various immunomodulatory strategies that target crucial inflammatory elements.

Except for ODE type of modeling, another type of mathematical modeling is the agent based modeling (ABM) approach. This type of modeling has been recognized as a useful approach to understand complex biologic processes (inflammation) (An 2001; Vodovotz, Clermont et al. 2004) and its underlying principle is the establishment of rules among the actors of the biological response (agents). Agent based model is an individual based computational model in that each rule is a probability of a given event to happen. An illustration to this is the agent-based model of inflammation and wound healing (cross-linked biological processes) presented in (Mi, Riviere et al. 2007). In this study the "agents" represent cells and cytokines that interact through the activation of local rules on a spatial grid of various probabilities. In essence, agent based models allow the "agents" to interact with each other and move in a region based on the activation of local rules. Another ABM type of modeling is discussed in (Alberti, Brun-Buisson et al. 2005) where there is emphasis on simulating the acute phonotrauma in cases with various cytokine 
profiles. In addition to this, in the studies done by An and collaborators (An 2001; An, Hunt et al. 2007; An 2008; An, Faeder et al. 2008), the applications of ABM in inflammation models have been effectively demonstrated. Therefore, such models shed useful insight on the interacting elements that present large heterogeneity. Both models (ABM and ODE) provide valuable insight regarding the behavior of complex systems. As such, there is no unidirectional answer to what modeling approach is the best as this is highly dependent on the scopes of each research task. However, it should be emphasized that ABM models need more computational power compared to ODE based models and ODE-based models are useful in applications where the overall behavior of an element (concentration of a mediator) is sought emphasizing the time-dependence of the interacting elements and offering opportunities for their future integration with agent-type models. In this research we are primarily interested in semi-mechanistic ODE based models in that we would like to simulate the complex dynamic behavior of the entire system (progression of a disease) through its elementary dynamic elements. However, the feasibility of an agent-based model of systemic inflammation in humans is also demonstrated in Chapter 5 (Section 5.5) of this dissertation.

\subsection{Clinical models of human endotoxemia}

Inflammation can be studied in the absence of complex pathophysiology and comorbidities of human sepsis by using surrogate models. Human endotoxin challenge is one well-accepted surrogate model for studying the acute inflammatory response as it captures many of the clinically observed features of systemic inflammation (Calvano, Xiao et al. 2005a; Fannin, Auman et al. 2005; Lowry 2005; Talwar, Munson et al. 2006; Wittebole, Hahm et al. 2007). Endotoxin, a major component of the outer membrane of 
gram-negative bacteria, activates the innate immune system, leading to inflammation. This moiety can be a complicating factor in a variety of situations including trauma, burns, invasive surgery and organ-specific illnesses. The prototypical examples of endotoxin are lipopolysaccharides (LPS), Figure 2.3.

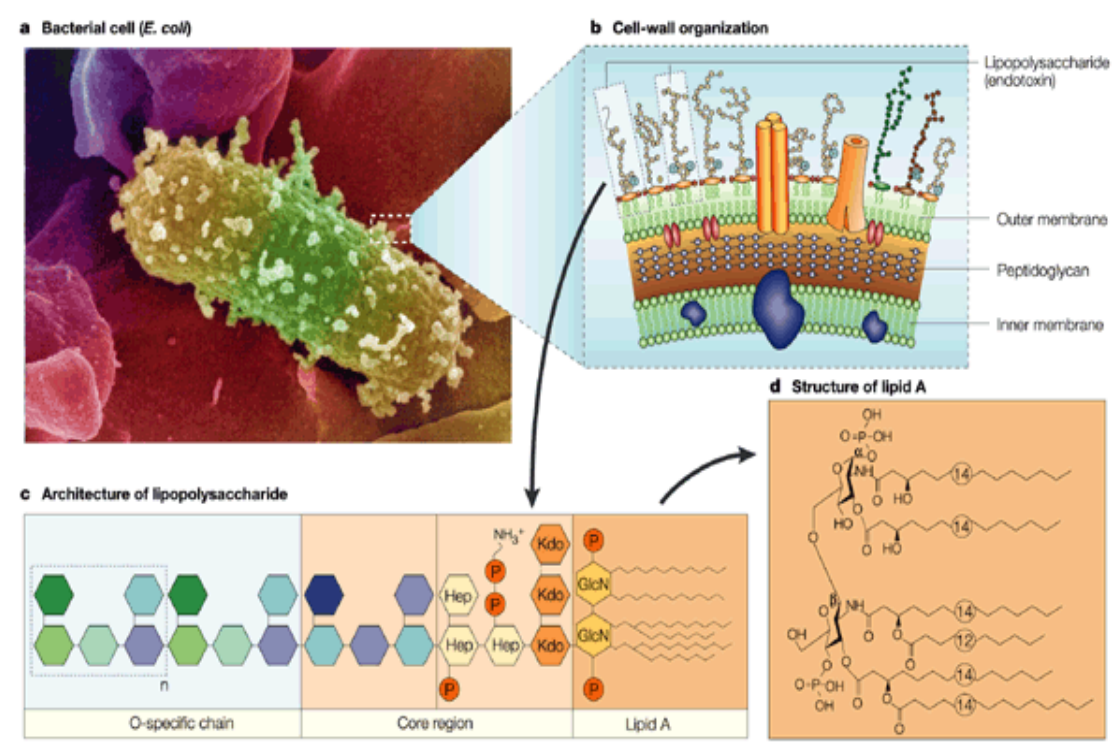

Figure 2.3: Electron micrograph of Escherichia Ecoli (E. coli) together with the schematic structure of lipopolysaccharide (LPS, endotoxin). The lipid A component is the primary structure of the toxic center of LPS (Beutler and Rietschel 2003).

Protruding from the outer membrane, LPS is composed of a lipid moiety referred to as lipid A and a polysaccharide chain called the O-chain that is partially extracellular. The inner portion of the O-chain consists of the core region that links the oligosaccharide to the lipid A moiety (Andreasen, Krabbe et al. 2008).

Studies involving experimental human endotoxemia have reported rapid intravenous infusion in doses of $2-4 \mathrm{ng} / \mathrm{kg}$ body weight, which effectively induce a monophasic systemic inflammatory response. The response following endotoxin administration in human subjects includes core temperature, cardiac, vasomotor, hematologic, metabolic, hormonal, acute phase reactant, and cytokine components that have been well described 
(Copeland, Warren et al. 2005; Lowry 2005; Santos and Wilmore 1996; van Deventer, Buller et al. 1990; Van Zee, Coyle et al. 1995). In particular, innate immune cell activation leads to the production and release of pro-inflammatory and anti-inflammatory cytokines (Opal and DePalo 2000), which are proximal mediators of the systemic inflammatory response (SIRS) and of the compensatory anti-inflammatory response syndrome (CARS), respectively, Figure 2.4.

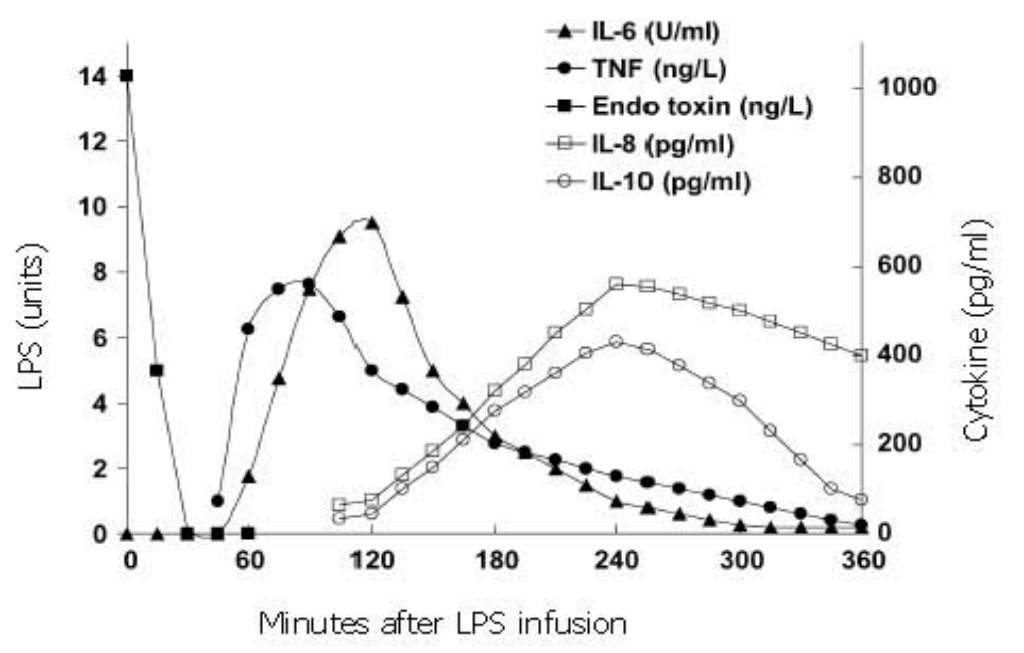

Figure 2.4: Endotoxin kinetics and mean expression of critical pro- and anti-inflammatory components following $2 \mathrm{ng} / \mathrm{kg}$ intravenous bolus of endotoxin. Adapted from (Lowry 2005).

In addition to transcriptional alterations, intravenous endotoxin administration in human subjects elicits a neuro-endocrine and hemodynamic response characteristic of acute injury and sepsis (Lin and Lowry 1998). Such similarities between the early, transient clinical phenotype and the inducible changes of human endotoxin model has led to the recognition that experimental human endotoxemia represents a reproducible model to evaluate the host response to acute illnesses (Lowry 2005). 


\section{Chapter 3}

\section{Significance}

Life sciences are facing a transition from descriptive to mechanistic approaches that reveal principles of cells, cellular networks, organs and their interactions across multiple scales of biological organization. The rapid progress of molecular biology in the wake of the identification of DNA's structure (Watson and Crick 1953) serves as a reminder of the power of reducing a system to its smallest possible components and studying them. Yet, although reductionism is powerful, its scope is limited. This is widely recognized in the study of complex systems whose properties are greater than the sum of their constituent parts (Aderem 2005). Recognizing that complexity, the emerging field of systems biology attempts to harness the power of mathematics, engineering and computer science to analyze and integrate data with the ultimate goal of creating models of entire biological systems. Thus, the recent growth of interest in systems biology reflects the increasing importance that integrative initiatives are being accorded in the biological sciences.

Central to the analysis of biological systems is the concept of the "network" defined as an interconnected group of systems and are potentially characterized by a critical property of complexity: emergence (Barabasi and Oltvai 2004). In the context of a biological system, the implication is that the macroscopic response (phenotype) of a system is the result of propagating information, in the form of disturbances, across an intricate web of interacting modules that span multiple biological scales. At the lowest level, there are interactions between molecular components of a cell, such as genes, 
RNA, proteins, and metabolites. These interactions define elementary building blocks that are organized into intracellular pathways and regulatory motifs, which in turn are integrated, through appropriate interactions, into interacting modules that eventually give rise to an organism's response, Figure 3.1.

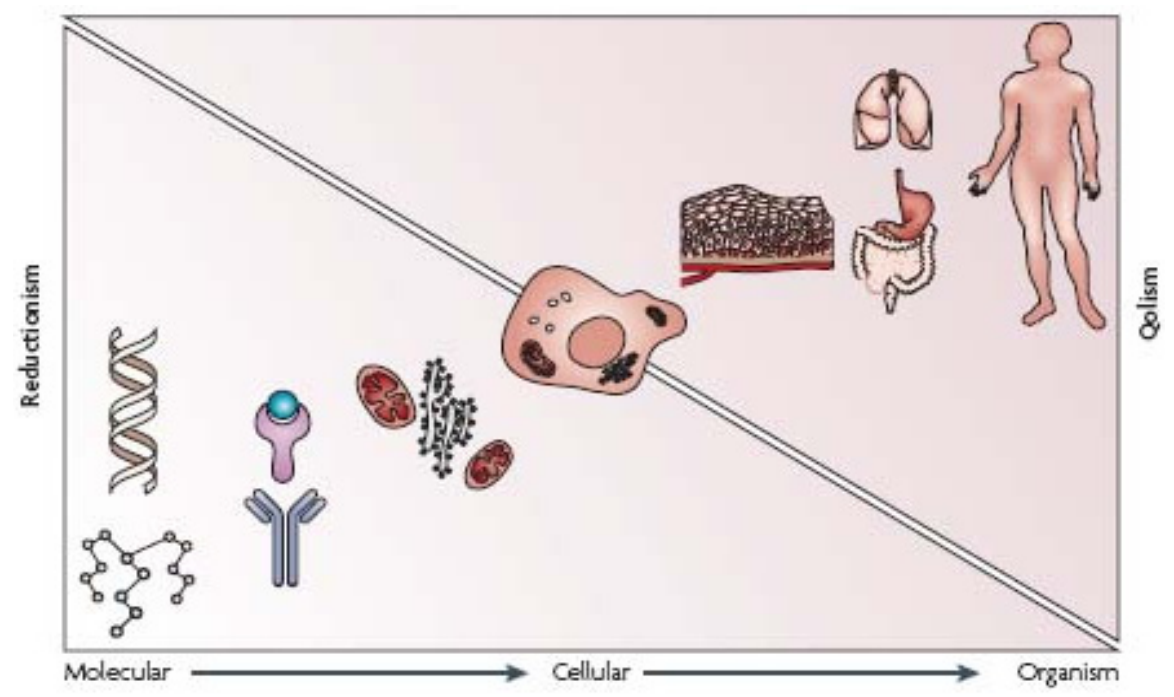

Figure 3.1: The state of a system is expressed in multiple scales of the biological organization. Changes at the genome (low) level lead to modified intracellular signaling which causes changes in cellular behavior and thereby gives rise to perturbations to higher biological scales (tissues, organs, entire organism). Focusing on the cell as the fundamental unit allows us to naturally link molecular reductionism with quantitative holism (“qolism”) (Anderson and Quaranta 2008).

The emergent behavior of a biological system, whether it relates to the control of the expression of a single gene (Babu, Luscombe et al. 2004) or the manifestation of a disease (Calvano, Xiao et al. 2005a) is the result of the coordinated action of network elements. It is therefore becoming apparent that in order to quantify such mechanisms one need to integrate multiple processes on multiple scales. The host response can be therefore considered as the emergence response of a network of interacting elementary signaling modules. Deciphering the connectivity and the dynamics of emerging network architectures becomes a critical question in the analysis of biological systems. The 
resurgence of methods that enable the analysis of such question is largely facilitated by the tremendous advances in monitoring changes at the cellular and molecular level driven primarily by developments in measuring gene expression at the genome-wide scale. Further, advances in "bedside" technologies monitor the stage of a disease by measuring vital signs defining the clinical outcome of the host response. With the technology maturing what started as an attempt to classify temporal patterns (Huang, $\mathrm{Hu}$ et al. 2009) has evolved into sophisticated analyses providing semi-mechanistic disease progression models (Chan and Holford 2001).

This dissertation presents a systems-level modeling approach that based on human experimental data that span multiple scales, explores the emergence of interaction networks at the cellular (low) level of signaling and regulation components that give rise to the overall systemic response. In this research, multiscale models of human endotoxemia, as prototype models of acute inflammation in humans, are developed that couple critical aspects of the complex bidirectional relationship between the CNS and the immune response linking extracellular signals with transcriptional dynamics, signaling cascades and physiological components. Driven by the premise to develop more mechanistic, physiology-based in-silico models of inflammation, the unique aspects that are explored in this research include:

(i) identification, based on the analysis of temporal gene expression responses, of the essential components characterizing cellular transcriptional dynamics in response to an external perturbation. We hypothesize that there exists a critical set of dynamic features that capture the essence of the cellular response exploring the 
idea that cellular responses correspond to dynamically converging highdimensional transcriptional trajectories (Yang, Almon et al. 2009b);

(ii) reverse engineering of quantifiable representations of these elements exploring the concept of physicochemical (Aldridge, Burke et al. 2006) and indirect response (IDR) modeling (Jusko and Ko 1994) that connect extracellular signals and intracellular signaling cascades leading to the emergent transcriptional dynamics. Physicochemical modeling seeks to describe essential biological processes in terms of equations that can be physiologically interpretable; thus creating dynamic repositories of interpretable knowledge. Such models work best with regulatory processes where components (i.e. "pathway signals") and connectivity are relatively well established. On the other hand, when prior knowledge is sparse, the manifestation of a perturbation is difficult to be explicitly described using elementary kinetic reactions. Our inability to precisely model such signaling events makes IDR appealing. The underlying assumption of indirect response models is that external signals affect indirectly the synthesis and/or degradation term of the response of interest; and finally

(iii) multiscale, physiology-based modeling that quantifies critical aspects of the neuro-immune crosstalk while clinical observables at the physiome level are explicitly incorporated to assess systemic decomplexification and abnormalities indicative of the physiologic status of the host.

The multiscale human inflammation model will be the ultimate deliverable of the proposed research allowing us to clarify how cellular events and inflammatory processes contribute to adverse clinical outcomes in acutely stressed patients. 


\section{Chapter 4}

\section{Transcriptional Analysis and Intrinsic Responses}

Intravenous administration of endotoxin elicits dynamic and reproducible changes in the circulating leukocyte population as well as significant changes in blood leukocyte gene expression patterns, with resolution of all clinical manifestations within 24hr (Calvano, Xiao et al. 2005a). The response following endotoxin administration in human subjects includes changes in core temperature, cardiac, vasomotor, hematologic, metabolic, hormonal, acute phase reactant, and cytokine components that have been well described (Copeland, Warren et al. 2005; Lowry 2005; van Deventer, Buller et al. 1990; Van Zee, Coyle et al. 1995). Innate immune cell activation leads to production and release of proinflammatory cytokines, which are proximal mediators of the systemic inflammatory response. Although the bulk of this pro-inflammatory mediator release likely originates in cells of the reticuloendothelial system (Fong, Marano et al. 1990), the leukocytes present in peripheral blood are also activated and, importantly, are available for sampling with minimal invasiveness.

In order to study the underlying complexity of the dynamics of inflammation and to establish quantifiable relationships among the various components of the inflammatory response, model-based approaches have been proposed (Chow, Clermont et al. 2005; Cross and Opal 2003; Lagoa, Bartels et al. 2006). A number of excellent prior studies(Chow, Clermont et al. 2005; Day, Rubin et al. 2006; Kumar, Clermont et al. 2004; Prince, Levy et al. 2006; Reynolds, Rubin et al. 2006; Vodovotz, Chow et al. 2006) have placed significant emphasis on simulating inflammation based on the kinetics of 
well-defined features of the overall response. One of the key characteristics of these models is the a priori postulation of certain components that are consistent with biological knowledge and are known to play a major role in triggering the inflammatory response (Chow, Clermont et al. 2005; Lagoa, Bartels et al. 2006). Thus there is emphasis on reducing the complexity of the models of inflammatory response by identifying a limited number of time-dependent interactions of key elements that are highly sensitive to specific modes of initiation and modulation of the response.

However, one of the big challenges is the systematic identification of such representative biological features, based on experimental data that can adequately represent the complex dynamics of a host undergoing an inflammatory response. This requires the decomposition of the non-linear dynamics of the response into an elementary set that can serve as a surrogate for predicting the collective behavior of the system. A possible answer to this problem can be identified through the analysis of gene expression data aimed at monitoring the dynamics of the host response to an inflammatory agent, exploring the idea that cellular responses correspond to dynamically converging highdimensional transcriptional trajectories (Huang, Eichler et al. 2005). Decomposing the intrinsic dynamics of the entire system into a reduced set of responses enables us to both project and understand the complex dynamics of the system by studying the properties of its essential dynamic parts. Given the transcriptional profiling analysis of human blood leukocytes, we hypothesized that the genes that are most responsive to LPS are governed by a definite mechanism and have concerted changes in their expression profile.

Gene expression data analyzed in this section were generated as part of the Inflammation and Host Response to Injury Large Scale Collaborative Project funded by 
the USPHS, U54 GM621119 (Cobb, Mindrinos et al. 2005). Human subjects were injected intravenously with endotoxin (CC-RE, Lot \#2) at a dose of 2-ng/kg body weight (endotoxin treated subjects) or $0.9 \%$ sodium chloride (placebo treated subjects). Following lysis of erythrocytes and isolation of total RNA from leukocyte pellets, (Calvano, Xiao et al. 2005a), biotin-labeled cRNA was hybridized to the Hu133A and Hu133B arrays containing a total of 44,924 probes for measuring the expression level of genes that can be either activated or repressed in response to endotoxin. A set of 5,093 probe sets were characterized by significant variation (corresponding to $0.1 \%$ false discovery rate) across the time course of the experiment using the SAM software (Storey, Xiao et al. 2005). The data are publicly available through the GEO Omnibus Database (http://www.ncbi.nlm.nih.gov/geo/) under the accession number GSE3284.

\subsection{Extracting essential inflammatory transcriptional responses}

We recently proposed a systematic computational framework that decomposes highdimensional microarray data into an elementary set of temporal responses (Yang, Maguire et al. 2007; Yang, Almon et al. 2008; Yang, Almon et al. 2009a). We are interested in unraveling a critical set of informative temporal responses that are characterized as the blueprints of the orchestrated dynamics of the perturbed biological system. In doing so, we hypothesize that there is a definite underlying mechanism that describes the emerging dynamic inflammatory response and capturing the essential inflammatory responses might serve as surrogates for the dynamic evolution of the host response due to endotoxin stimulus. Based on our prior work, we first apply a microclustering approach, which is based on a symbolic transformation of time series data which assigns a unique integer identifier (hash value) to each expression motif (Yang, 
Maguire et al. 2007). The symbolic transformation of the expression motifs and the subsequent assignment of hash values to each expression profile (Yang, Maguire et al. 2007) produces a distribution of motif values for all the available probes, Figure 4.1.

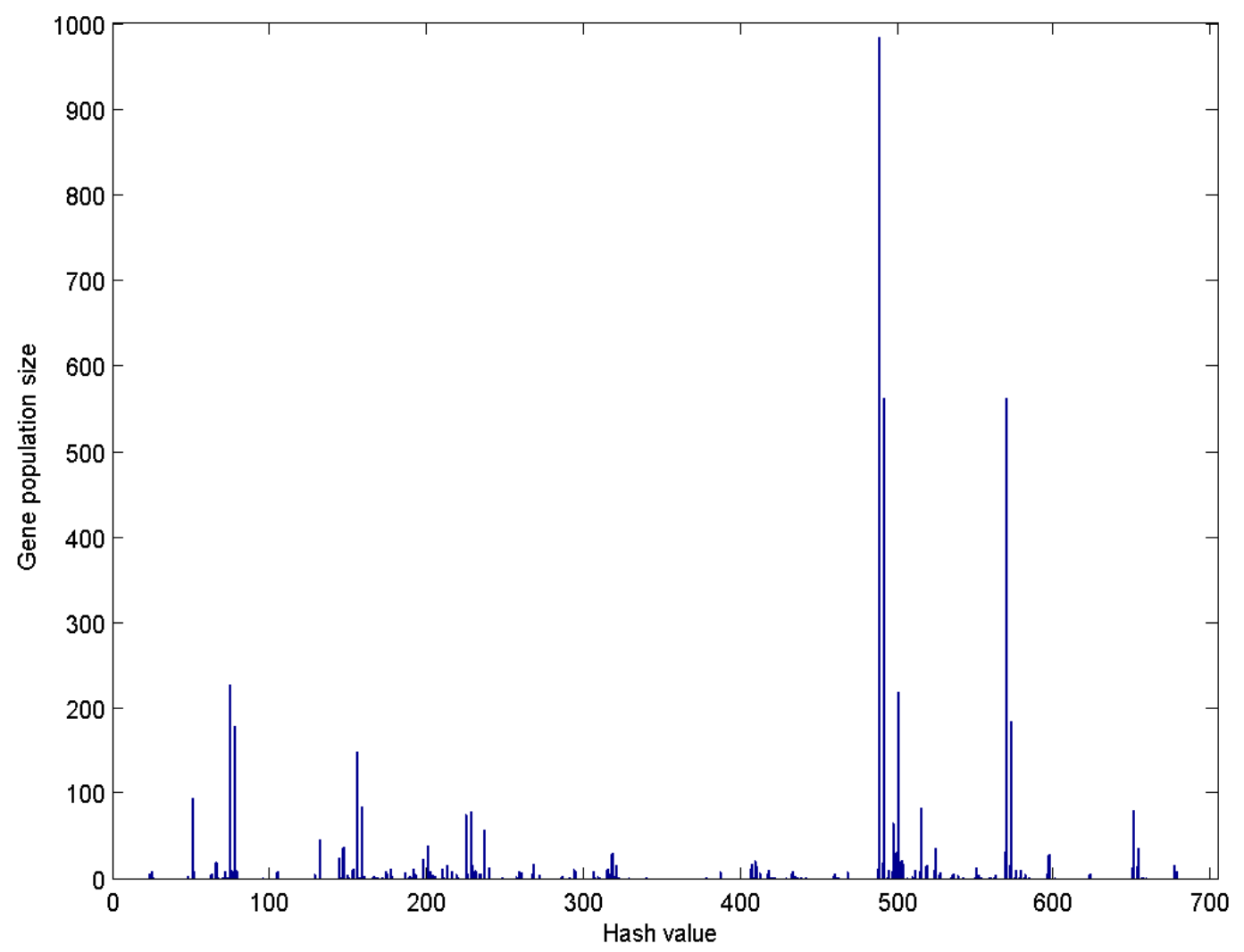

Figure 4.1: Number of probe sets in each expression motif of inflammatory transcriptional signatures of human blood leukocytes. A histogram of hash values is used to visualize the number of probe sets in each micro-cluster. 5,093 probe sets are micro-clustered to 224 expression motifs. 
Having assigned the temporal expression profiles to distinct motifs, the next task is to select expression motifs that appear to be highly non-random based on their gene population size. In order to estimate a p-value for each expression motif we generate random data with the same dimensions as the original dataset $(5,093$ probe sets and 6 time points). Genes that hash to the same integer value for the random data are characterized by a distribution that approximately follows an exponential decay and subsequently we can estimate the cumulative distribution for an exponential model. Thus, an appropriate $\mathrm{p}$-value $=1 /$ (total number of expression motifs $)$ which equals 0.0045 , is used to evaluate highly non-random expression motifs (clusters) Figure 4.2. The analysis generates a sub-set of 16 transcriptional motifs which are considered to be statistically significant in terms of their population size, Figure 4.3. Therefore, this family of expression motifs, and the associated probe sets, is most characteristic of the exposure of the host to LPS.

Having identified the statistically significant expression motifs from the initial large set of micro-clusters we need to identify a discriminating set of critical temporal shapes that best characterizes the intrinsic dynamic response of the system. In doing so, we explore the concept of Transcriptional State (TS) previously introduced in (Yang, Maguire et al. 2007). We define the TS of the system as the overall distribution of expression values at a specific time point by quantifying the deviation of the system at each time point versus a baseline distribution $(\mathrm{t}=0 \mathrm{hr})$ applying a Kolmogorov-Smirnov test (Lampariello 2000), Figure 4.4. 


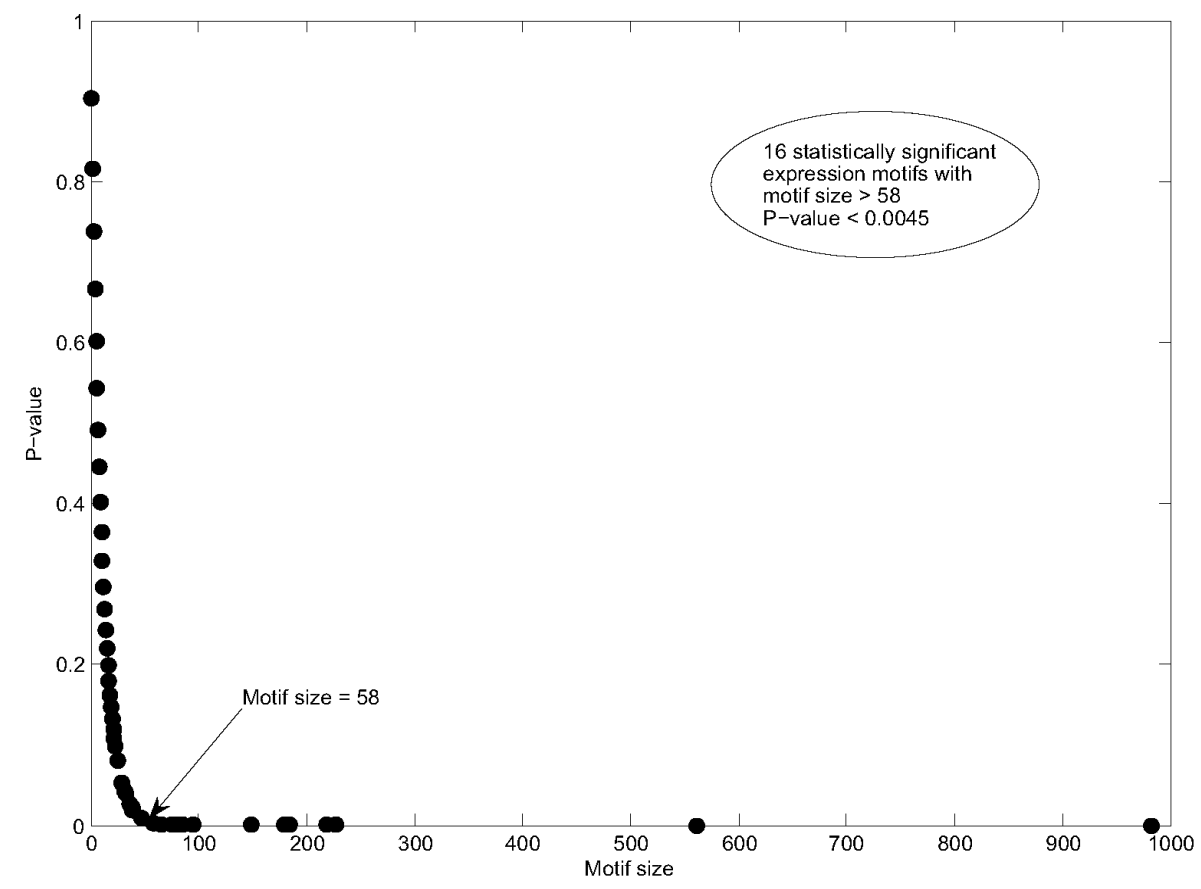

Figure 4.2: Estimated p-value vs. expression motif sizes. Expression motifs with size $\geq 58$ corresponding to $p$-value $<1 / 224$ (total number of probe sets in informative motifs).
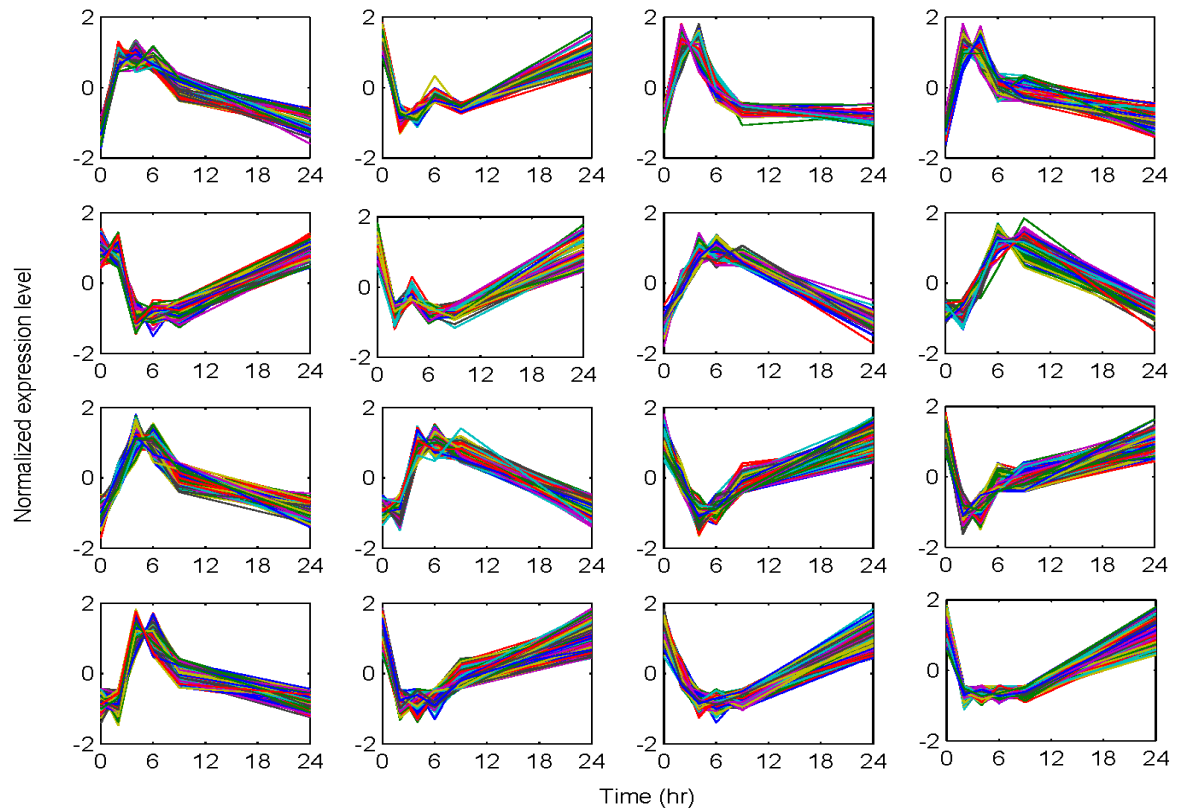

Figure 4.3: Temporal profiles of statistically significant expression motifs. Normalized expression values of motifs with p-value $<0.0045$ (with respect to cluster size) vs. time. 

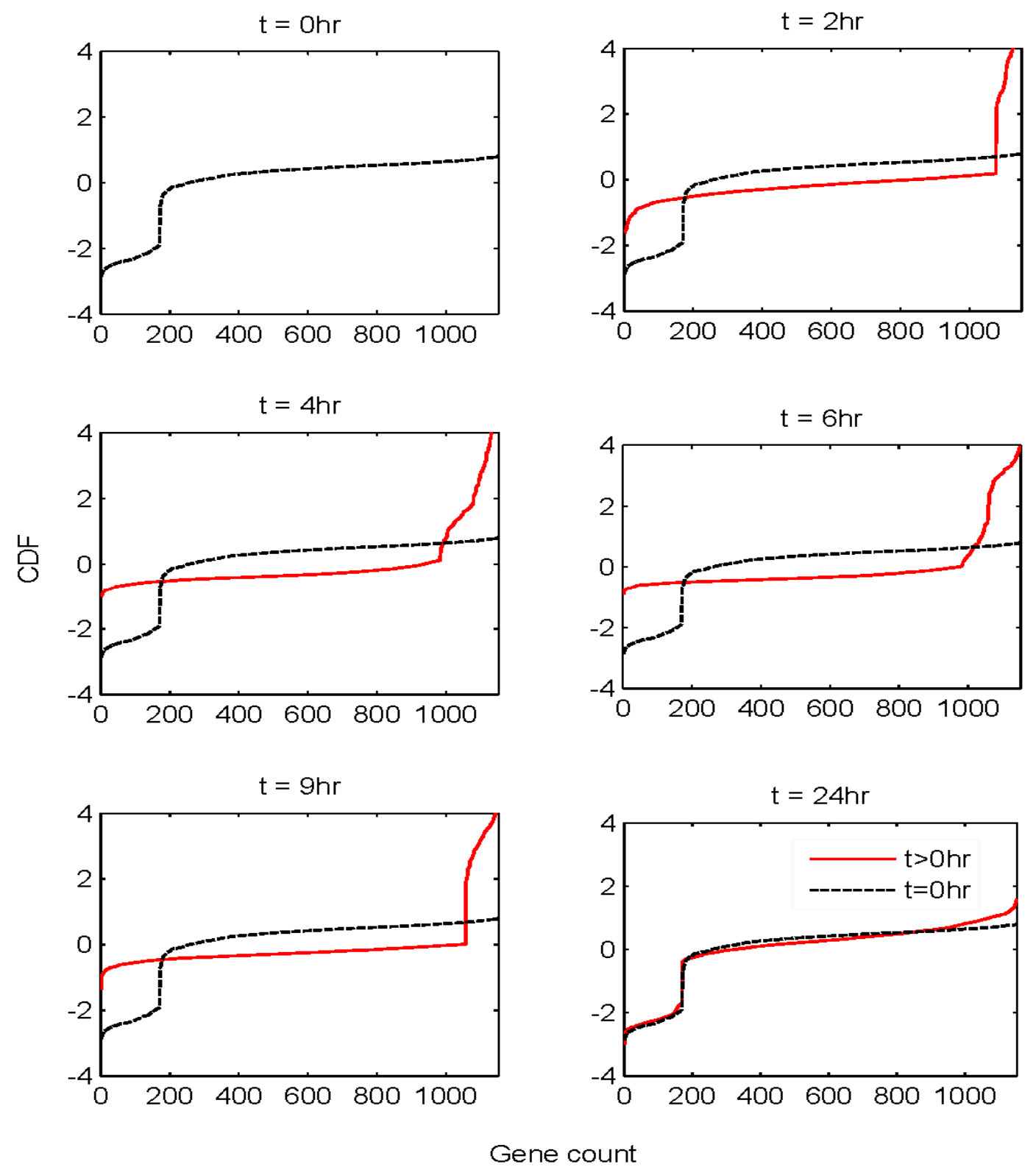

Figure 4.4: Estimation of the Transcriptional State of the System. Distribution of expression values for all genes at each time point $(\mathrm{t}>0 \mathrm{hr})$ versus the baseline distribution $(\mathrm{t}=0 \mathrm{hr})$. 
The distribution of expression values for all genes at each time point $(\mathrm{t}>0 \mathrm{hr})$ versus the baseline distribution $(\mathrm{t}=0 \mathrm{hr})$ is illustrated in Figure 4.4. The difference between these two distributions can be quantified (Kolmogorov-Smirnov test) which enables us to capture the inflammatory trajectory of the host response due to bacterial endotoxin. Therefore, there is a transcriptional deviation that onsets at $\mathrm{t}=2 \mathrm{hr}$ and progresses dynamically while returning to its original state at $\mathrm{t}=24 \mathrm{hr}$ (inflammatory resolution). Such an analytical approach allows us to identify coherent expression motifs that account for the intrinsic dynamics of the host.

Based on the definition of TS of the system the principal task is to extract from the entirety of the measured responses a critical set that defines the essential cellular response of the system. This selection is a combinatorial optimization problem for which we apply a stochastic optimization algorithm, based on simulated annealing (SA) (Kirkpatrick S. 1983). We run simulated annealing parametrically with respect to the number of expression motifs in order to identify the minimum number of informative motifs, Figure 4.5. The basic assumption is that due to an external disturbance, i.e., LPS administration, the system is perturbed from homeostasis and eventually, once LPS is cleared and the inflammatory reaction is eliminated, the host returns to the original state. Due to global nature of the transcriptional measurements and the fact that we do not a priori select a limited set of responsive genes, the entirety of the transcriptional response is expected to exhibited a rather Gaussian type of response with no clear defining responses (Vemula, Berthiaume et al. 2004). We have, however, previously demonstrated that through the use of the concept of TS it is possible to "tease out" the essential components of the cellular response in response to an external disturbance. 


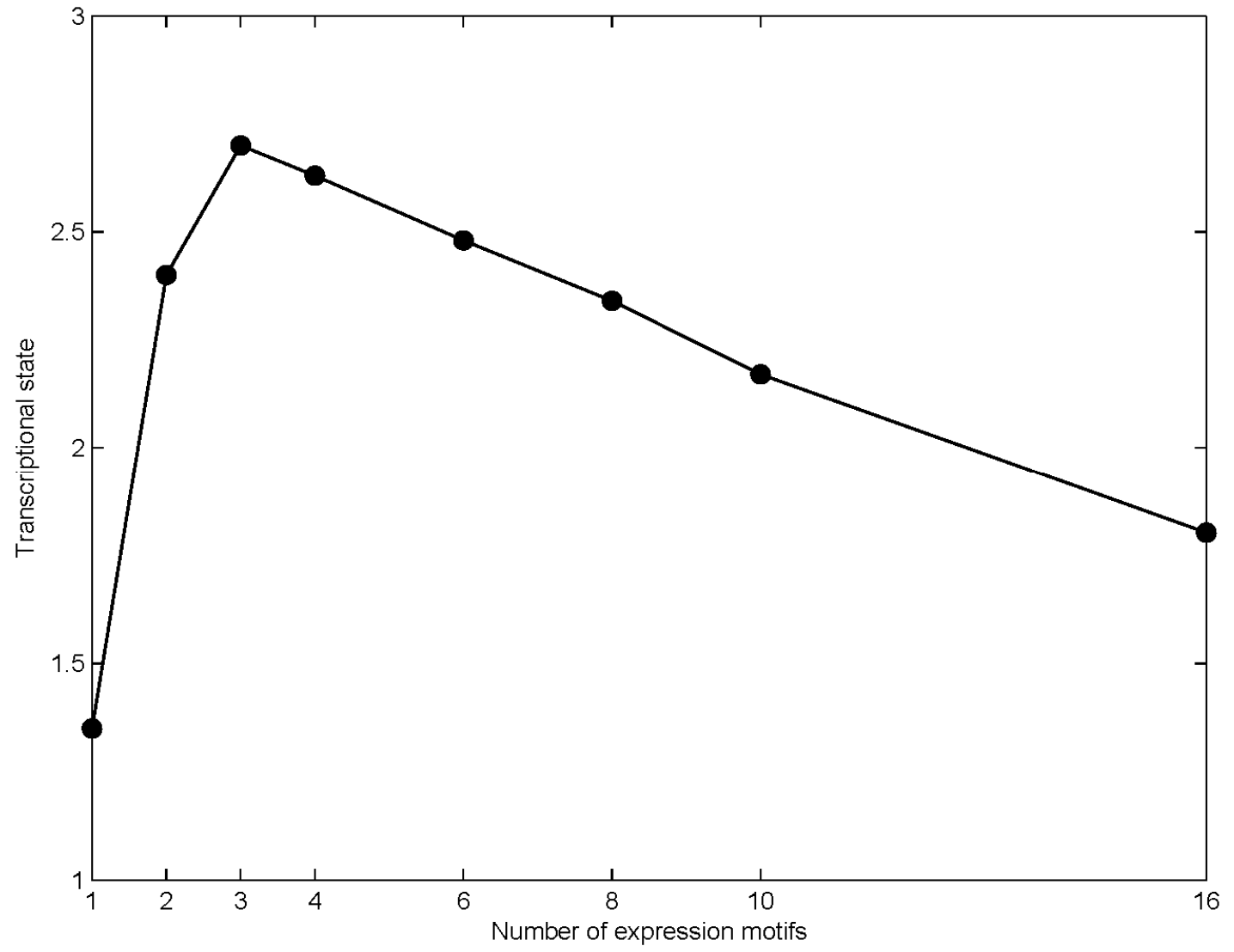

Figure 4.5: Deviation of the Transcriptional State of the system vs. number of expression motifs. The maximum perturbation in the intrinsic dynamics of the system occurs for 3 distinct expression motifs. 
Thus, given the basis set of 16 statistically significant motifs we identify the minimum number of expression motifs that account for the maximum deviation of the system from its baseline-homeostasis (Transcriptional State). As seen in Figure 4.5 the maximum deviation from homeostasis is observed for three motifs, whereas further addition of motifs reduced the deviation indicating the addition of less critical responses. Therefore, this result illustrates the existence of distinct critical sets of temporal responses that capture the intrinsic dynamics of the host response to endotoxin administration, Figure 4.6. The first response (illustrated by red color) is characterized by an early increase in the gene expression level during the first $2 \mathrm{hrs}$ after the endotoxin challenge, whereas the second essential response (green color) shows an increase at the gene expression level at a later time event (4hrs $-6 \mathrm{hrs})$. The third response (represented by blue color) is characterized by a downregulation during the time course of the experiment and eventual return to baseline at $24 \mathrm{hrs}$. All responses resolve, i.e. return to baseline $24 \mathrm{hr}$ post-exposure, which is in agreement with the overall design of the study and the reversible nature of the elicited response. Having de-convoluted the inflammatory signal into its essential components, it is hypothesized that genes whose transcriptional signatures are highly correlated with the essential responses account for the maximum deviation of the system from its baseline (homeostasis) and thus play a major role in the dynamic evolution of the inflammatory process. The biological relevance of the intrinsic responses is identified by evaluating the enrichment of the corresponding subsets in inflammation-specific pathways using ARRAYTRACK (Tong, Cao et al. 2003). 


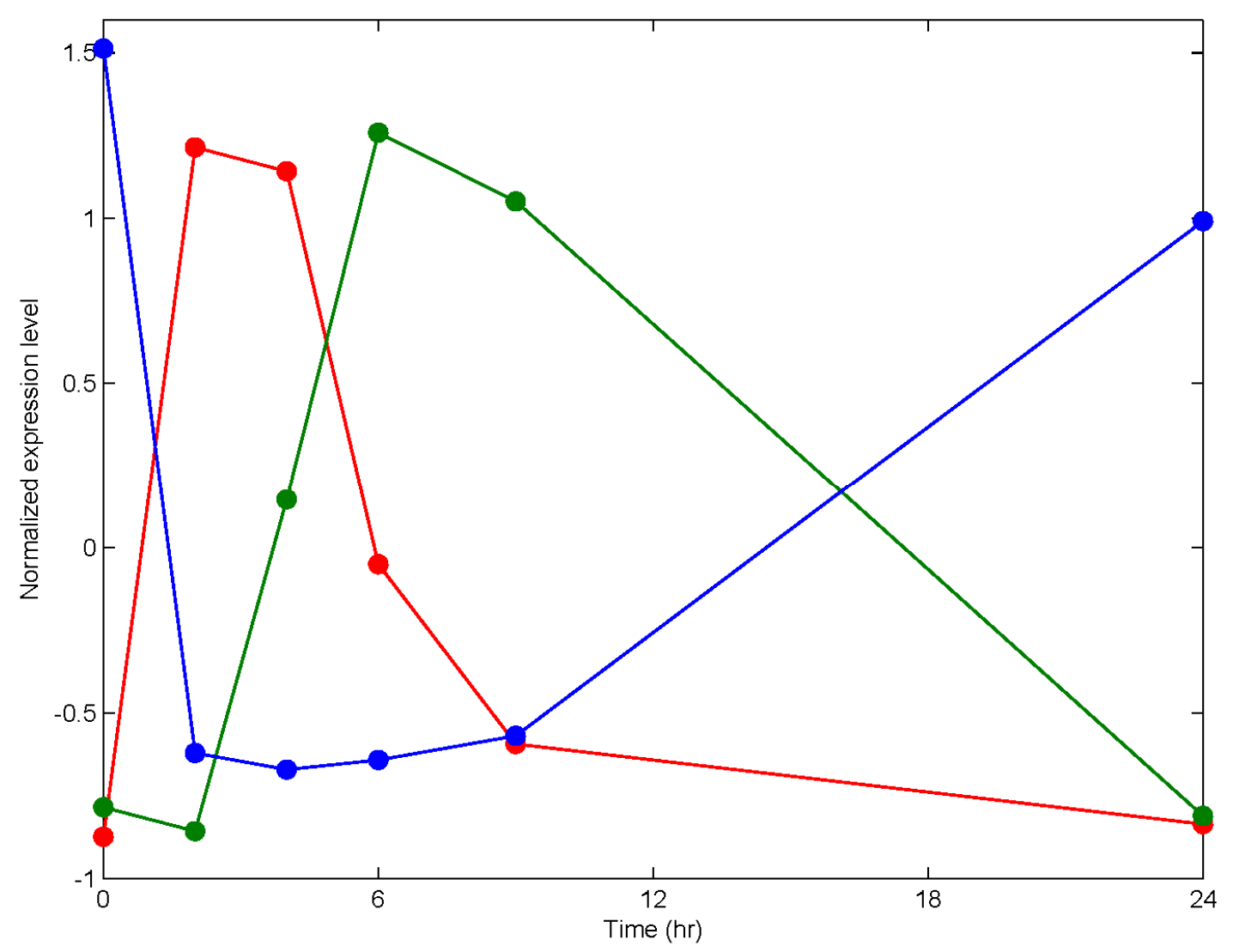

Figure 4.6: Essential leukocyte transcriptional elements: (red color) Pro-inflammatory response (P), (green color) Anti-inflammatory response (A) and (blue color) Energetic response (E). 


\subsection{Functional characterization of essential responses}

Upon identification of the probe sets composing these three essential transcriptional responses we identify significant localization in relevant biological pathways. We identify three critical expression motifs enriched in critical and relevant biological pathways: (i) Early up-regulation response (Pro-inflammatory component, P): Genes in this major temporal class are important in cytokine-cytokine receptor interactions (C-X-C motifs and cytokines - CXCL1, CXCL2, CCL20) as well as in Toll like receptor signaling pathway (CCL4, IL1B, IL8) crucial in activating transcription factors that act synergistically with pro-inflammatory transcription factors such as members of NFkB/RelA family; (ii) Late up-regulation response (Anti-inflammatory component, A): Genes in this functional class participate in the JAK-STAT cascade (IL10RB, JAK3, STAT2, STAT5B) which is essential to regulate the expression of target genes that counter-react the inflammatory response. In addition to this, it is emphasized (Murray 2007) that a STAT pathway from a receptor signaling system is a major determinant of key regulatory systems, including feedback loops such as SOCS induction which subsequently suppresses the early induced cytokine signaling and essential activators for IL10 signaling (Brightbill, Plevy et al. 2000). Moreover, we identified the late increased expression of IL10RB which is assumed to be indicative of the IL10 signaling cascade; and (iii) Down-regulation response (Energetic component, E): The down-regulated essential response is characterized by a set of genes, which are mainly involved in the cellular bio-energetic processes. In addition to this, a large set of genes, which are essential to ribosome biogenesis and assembly (RPL/RPS family) are repressed coupled with those genes, which participate in protein synthesis machinery, oxidative 
phosphorylation (ATP5A, COX11, NDUFA11) and pyruvate metabolism (PDHB, PDHX, MDH1). Endotoxin-induced inflammation causes the dysregulation of leukocyte bioenergetics and a persistent decrease in mitochondrial activity leads to reduced cellular metabolism and a subsequent decline in organ function (Singer, De Santis et al. 2004). A restoration of organ function should be associated with an increase in bioenergetics and metabolic activity (Brealey, Brand et al. 2002) and we are assuming that a persistent shut down of these genes might lead to multiple organ dysfunction.

These transcriptional responses effectively decompose the overall dynamic and present the constitutive elements of the overall response. The computational integration of these components is discussed in the following chapter (Chapter 5). In order to reproduce the experimental data, we select the transcriptional signature of specific genes representative of each essential response. IL1B is selected to serve as the representative biomarker of the pro-inflammatory response. The gene transcript of IL10RB is considered to be indicative of the immune-regulatory signal of the anti-inflammatory response. Finally, a subunit of NADH ubiquinone dehydrogenase complex (mitochondrial component) NDUFC2 is considered as the proxy for the energetic component. These essential transcriptional signatures are normalized by taking the ratio of the measured mRNA level at each time point with respect to the control time point $(\mathrm{t}=$ 0hr), Figure 4.7. Selecting any other gene that belongs to the aforementioned essential inflammatory responses can very well be used as a surrogate for a representative of the response and will not alter the qualitative characteristic of our semi-mechanistic mathematical model to be described in the following chapter. 


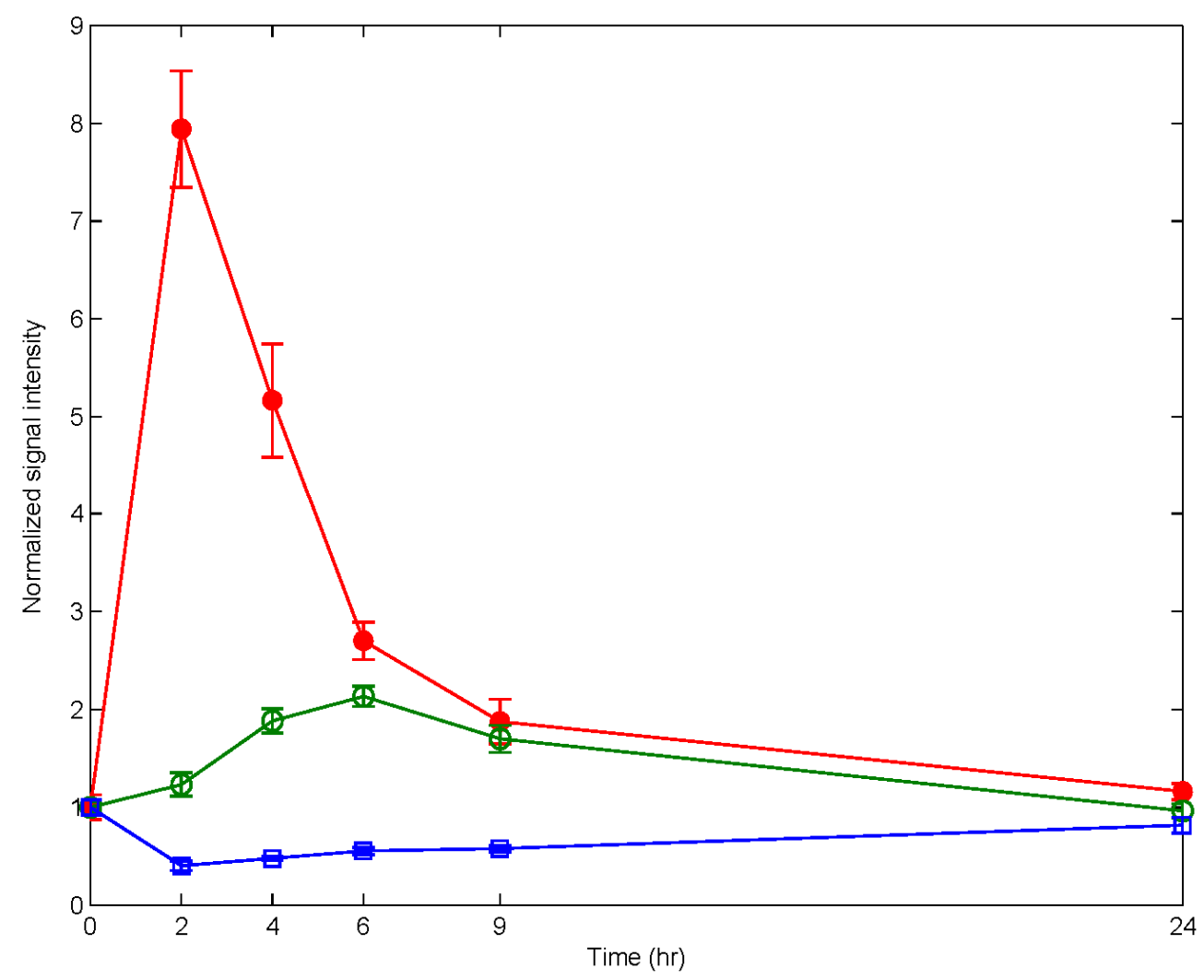

Figure 4.7: Transcriptional signatures of marker inflammatory genes: (red color) mRNA of IL1B representative of the essential pro-inflammatory response. (green color) mRNA of IL10RB indicative of the late anti-inflammatory response and (blue color) mRNA of NDUFC2 which is considered as the proxy for the energetic component. Data are represented as mean $\pm \mathrm{SEM}$. 


\section{Chapter 5}

\section{Cellular Level Physicochemical Models of Inflammation}

Considering the leukocytes as a well defined system, the purpose of human studies like the ones described in the previous chapter is to characterize qualitatively the cellular dynamics. The purpose of a "systems biology" approach, on the other hand, is to reverse engineer quantifiable representations of the intracellular dynamics (Zamir and Bastiaens 2008) by identifying (i) appropriate constitutive elements; (ii) the topology of the interactions among these elements; and (iii) the quantitative relations among these elements. In Chapter 4 we addressed the first issue, whereas now we will discuss how to construct the topology and dynamics of the underlying network describing the dynamics at a single scale, i.e, that of the leukocytes. Vodovotz and coworkers recently discussed the state of the art in mechanistic simulations of inflammation (Vodovotz, Constantine et al. 2009).

We have recently advocated a cellular, mechanistic-based, modeling approach which explores three unique aspects. First, through the analysis of the leukocyte gene expression data we identify the essential responses characterizing the cellular (transcriptional) dynamics as described. Second, we explore the concepts of physicochemical modeling (Aldridge, Burke et al. 2006) to express the intertwined relations and dynamics that connect extracellular signals and intracellular signaling cascades eventually leading to the emergent transcriptional dynamics (identified in the previous step). Finally, we explore the pharmacodynamic concept of indirect response (IDR) (Jusko and Ko 1994) in order to establish implicit interaction among signaling 
molecules and emerging transcriptional responses. In this section we will summarize the basic concepts and models.

\subsection{An indirect response model for human endotoxemia}

In our injury model, the inflammatory response is activated when endotoxin is recognized by pathogen recognition receptors (Wells, Ravasi et al. 2005). The LPS-induced stimulation initiates a complex signaling cascade that ultimately targets the transcription initiation of pro-inflammatory cytokines (Kishore, McMullen et al. 2004). During the recognition process LPS binds to the LPS-binding protein in plasma and is delivered to the surface receptor CD14. Subsequently, LPS is transferred to the signaling receptor tolllike receptor 4 (TLR4) with the recruitment of its essential accessory protein MD2 (Du, Poltorak et al. 1999; Guha and Mackman 2001; Van Amersfoort, Van Berkel et al. 2003). Downstream of the receptor-ligand complex there are intermediate secondary messages that involve both the amplification of the signaling and its diversification in the cytoplasmic region so that ultimately such a signal transduction cascade will activate critical signaling modules that will subsequently lead to the translocation (activation) of pro-inflammatory transcription factors (i.e. NF-kB) responsible for the transcription initiation of inflammatory genes. Therefore LPS interacts with its signaling receptor (TLR4) in order to induce the signal transduction cascade that triggers essential signaling modules for the activation of pro-inflammatory transcription factors. This cascade of events is eventually manifested through the coordinated transcriptional changes measured via high-throughput microarray analyses (Calvano, Xiao et al. 2005b).

Due to our inability to precisely model such a cascade of events using elementary kinetic steps we will assume that the effect of the extracellular inflammatory signal (LPS) 
in initiating the transcriptional machinery is indirect. Thus we propose to model such a transcriptional event using the basic principles of an Indirect Response Model (IDR) widely used in developing pharmacodynamic and pharmacogenomic models (Jin, Almon et al. 2003; Krzyzanski and Jusko 1997). Despite the fact that an IDR model does not take explicitly cross-talk interactions into account, the principles of IDR approach can be extended to describe transduction processes and time dependent disease processes. Therefore, in order to establish quantifiable relationships among the previously identified "essential" components of human inflammation, an indirect response model is proposed and developed in (Foteinou, Calvano et al. 2007; Foteinou, Calvano et al. 2009a).

\subsubsection{Elements of an indirect response model of acute human inflammation}

We consider each essential transcriptional motif to be the manifestation of a process involving a synthesis and a degradation term. The underlying assumption of IDR models is that external signals affect indirectly the synthesis and/or degradation term of the response of interest, in our case the transcriptional dynamics. In the most general case it is assumed that the synthesis, or production, follows $0^{\text {th }}$ order kinetics, whereas the degradation follows $1^{\text {st }}$ order kinetics (Derendorf, Lesko et al.). As a result, the existence of such signals can either stimulate or inhibit the production and degradation rate of the response. Therefore, we assume that the upstream activated ligand-receptor signaling complex serves as the intracellular signal that indirectly will stimulate the production rate of transcriptional effects associated with the pro-inflammatory response as well as with the transcriptional activation of the gene transcript of the receptor $(\mathrm{mRNA}, \mathrm{R})$.

The binding interaction between the endotoxin (LPS) and the receptor (R) is assumed to be a standard ligand-receptor interaction (Lauffenburger and Linderman 1996). The 
activated ligand-receptor complex triggers an intracellular signal (DR*) which indirectly stimulates the production rate of the pro-inflammatory response (P). This proinflammatory response can be characterized as the "first-line" transcriptional response that is triggered upon the recognition of the extracellular ligand (LPS) by the pattern recognition receptors (PRR i.e. TLR4) (Aderem and Smith 2004). Pro-inflammation will serve as the signal that will further stimulate the downregulation of genes that are associated with the cellular energetic processes (Protti and Singer 2007). We hypothesize that the pro-inflammatory response acts as the stimulatory factor for the energetic response whilst a dysregulation in the cellular bio-energetics can serve as a positive feedback danger signal to the pro-inflammatory response.

The anti-inflammatory response serves as the essential immunoregulatory signal that aims at restoring homeostasis in the host defense system. Thus, it will be stimulated by the activation of the inflammatory components which are the pro-inflammation and the energetic response. Thus, it will serve as the inhibitory signal on the production rate of the pro-inflammation and the energetic response. We are assuming that it will negatively regulate the TLR pathway (Aderem and Smith 2004) modeling it as inhibition of the activated intracellular signal DR*. The essential hypothetical elements of the transcriptional response induced upon recognition of LPS are shown in Figure 5.1. 


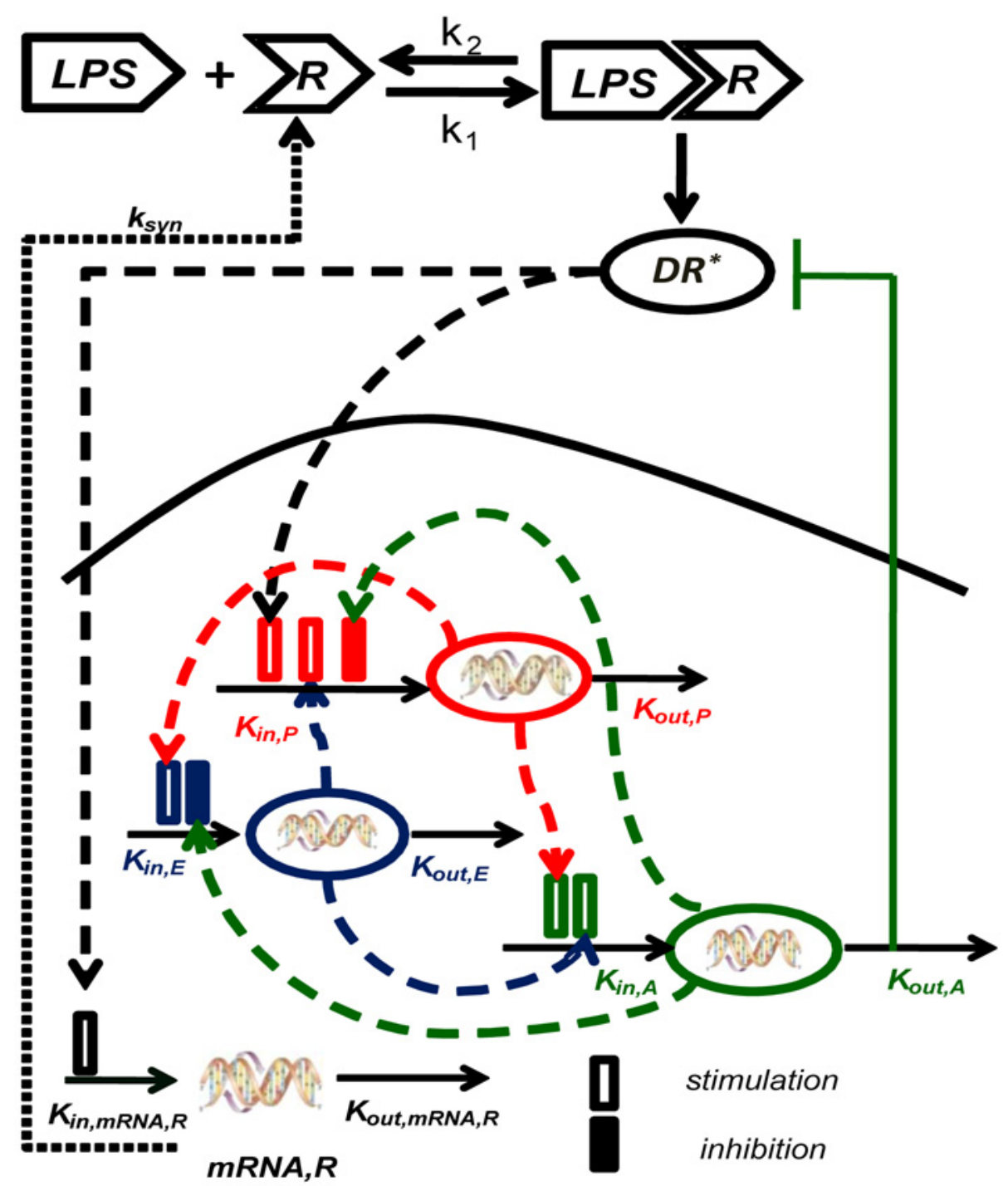

Figure 5.1: Notional modeling framework of LPS response. Upon binding to its receptor a signaling cascade is activated which leads to the up/down-regulation of numerous pro- and anti-inflammatory genes. LPS binds to the receptor (R) and forms the complex (LPSR) while it activates the signaling complex $\left(\mathrm{DR}^{*}\right)$ which indirectly stimulates the production rate $\mathrm{K}_{\mathrm{in}, \mathrm{P}}$ of the pro-inflammatory $(\mathrm{P})$ response. The proinflammatory response indirectly stimulates the production rate of the energetic $(E)$ response $\left(K_{\mathrm{in,E}}\right)$ and the production rate of anti-inflammatory $(A)$ response $\left(\mathrm{K}_{\mathrm{in}, \mathrm{A}}\right)$. The energetic response will stimulate both proinflammation and anti-inflammation whilst anti-inflammation will serve as the immunoregulatory component of the system restoring homeostasis intracellularly. 


\subsubsection{Developing an indirect response model}

The dynamics of the inflammatory stimulus (LPS) are described in Eq. (5.1) as a convolution of two terms: a first order elimination with rate $\mathrm{k}_{\mathrm{lps}, 2}$ and a logistic-type function with growth rate $\mathrm{k}_{\mathrm{lps}, 1}$. Effectively despite the presence of various mediators that are activated in response to LPS (i.e. LPS binds to LBP plasma protein during its recognition from the host) we model a single compartment pharmacodynamics model for LPS assuming a homogeneous circulating blood compartment. The logistic function is usually used to model a variety of physical situations in which a quantity's growth is "self-limited" which means that the initial growth is approximately exponential and as saturation begins the growth stops (Zwietering, Jongenburger et al. 1990). Therefore, depending on the relative magnitude of the two rate parameters of the clearance of LPS we can simulate situations where the bacterial concentration is not fully eliminated. In human subjects endotoxin is cleared within the first $2 \mathrm{hrs}$ of post-LPS administration with an approximate average half time $\tau_{1 / 2} \sim 8-15$ min (Greisman, Hornick et al. 1969). The two parameters $\mathrm{k}_{\mathrm{lps}, 1}$ and $\mathrm{k}_{\mathrm{lps}, 2}$ have been independently estimated so that the LPS profile decays within two hrs in the absence of any complications.

The dynamics of the TLR4 receptor (R), Eq. (5.2), depend on the association/dissociation parameters of the ligand-receptor interaction (Lauffenburger and Linderman 1996) whose the corresponding parameters $\mathrm{k}_{1}$ and $\mathrm{k}_{2}$, are based on literature values (Shin, Lee et al. 2007) and the translation of its $\mathrm{mRNA}_{\mathrm{R}}$ to surface protein $\left(\mathrm{k}_{\mathrm{syn}}\right)$. The rate of translation $\mathrm{k}_{\mathrm{syn}}$ of the $\mathrm{mRNA}_{, \mathrm{R}}$ to the corresponding surface protein describes the dynamic evolution of synthesis of new receptors; hence this parameter is estimated so that the dynamic profile of the surface free receptor is down-regulated based on the 
premise that under the inflammatory stimulus the surface free receptors are occupied. The dynamics of the gene transcript of the receptor, Eq. (5.3), is characterized by a production rate $\left(\mathrm{K}_{\mathrm{in,mRNA}, \mathrm{R}}\right)$ and a degradation rate $\left(\mathrm{K}_{\text {out,mRNA,R }}\right)$ but is assumed to be indirectly stimulated by the convoluted activated $\mathrm{DR}^{*}$ signal as shown in Figure 5.1. Experimentally, the transcript of the receptor is characterized by an up-regulation for the first 4 hrs post-LPS administration and then returns to baseline (Bosisio, Polentarutti et al. 2002).

The dynamics of the equilibrium complex (LPSR) is characterized by the binding parameters $\mathrm{k}_{1}, \mathrm{k}_{2}$ and the parameter $\mathrm{k}_{3}$ which characterizes the rate of formation of the activated signaling complex, DR*, Eq. (5.4). The formation of the activated signaling complex $\left(\mathrm{DR}^{*}\right)$ is proportional to the equilibrium complex with a rate constant $\mathrm{k}_{3}$ and it decays with rate $\mathrm{k}_{4}$, Eq. (5.5). However, we assume that the essential anti-inflammatory component will indirectly regulate the activated intracellular signaling complex. Such a negative feedback serves the purpose of incorporating a regulatory effect on the intracellular activated signaling complex once the transcriptional response has been initiated. In addition to this, the non-linear Hill type function serves the purpose of modeling a bistable behavior of the system (Xiong and Ferrell 2003). Such a bistability is an essential characteristic of the non-linear dynamics of inflammation as suggested from various animal studies (Kerschen, Fernandez et al. 2007; Lehmann, Freudenberg et al. 1987; Rifkind 1967; Tschaikowsky, Schmidt et al. 1998; Wang, Bloom et al. 1999). An increase in the dose of the inflammatory stimulus can be responsible for an overwhelming inflammatory response. We should emphasize that the exponent coefficient of the non-linear function in Eq. (5.5) does not quantitatively correspond to a 
Hill coefficient; instead it can be characterized as an ultrasensitive parameter which is associated with the bistable behavior of the system. What is more, the functional form of the activated signaling complex (DR $)$ in Eq. (5.5) allows us to model an improper (uncontrolled) TLR4 signaling even though the inflammatory stimulus (LPS) has been completely eliminated from the system. Given the role of TLRs in inducing strong inflammation improper regulation of this signaling pathway may also be involved in inflammatory diseases (Bhattacharjee and Akira 2006).

At the transcriptional response level the convoluted activated signal complex (DR $\left.{ }^{*}\right)$ indirectly stimulates the production rate of the essential pro-inflammatory response (P) which quantitatively is expressed by the linear function $\left(\mathrm{H}_{\mathrm{P}, \mathrm{DR}}{ }^{*}\right)$, Eq. (5.6). We also assume that the energetic response variable will be responsible for more pronounced inflammation and thus stimulates the pro-inflammatory response $\left(H_{\mathrm{P}, \mathrm{E}}\right)$. The antiinflammatory signaling component is assumed to inhibit the production rate of the proinflammatory transcriptional signature.

The anti-inflammatory signal (A) is stimulated by the activated pro-inflammatory response $\left(\mathrm{H}_{\mathrm{A}, \mathrm{P}}\right)$ as well as by the energetic response $\left(\mathrm{H}_{\mathrm{A}, \mathrm{E}}\right)$ and it decays with rate $\mathrm{K}_{\mathrm{out}, \mathrm{A}}$, Eq. (5.7). Finally, the energetic response (E) is indirectly stimulated by the proinflammatory response (P) and the anti-inflammatory component (A) indirectly counter-regulates both inflammatory components, i.e., the pro-inflammation and the energetic response of the system, Eq. (5.8). The parameters associated with the production and degradation rate of each essential transcriptional signature are estimated in order to best predict the essential responses with their experimental measurements. Equations (5.1) - (5.14) denote the functional forms of the indirect response. It is 
important to realize that special effort was placed in order to avoid high non-linearities in the system.

$$
\begin{aligned}
& \frac{\mathrm{dLPS}}{\mathrm{dt}}=\mathrm{k}_{\mathrm{lps}, 1} \cdot \mathrm{LPS} \cdot(1-\mathrm{LPS})-\mathrm{k}_{\mathrm{lps}, 2} \cdot \text { LPS } \\
& \frac{\mathrm{dR}}{\mathrm{dt}}=\mathrm{k}_{\mathrm{syn}} \cdot \mathrm{mRNA}, \mathrm{R}+\mathrm{k}_{2} \cdot(\text { LPSR })-\mathrm{k}_{1} \cdot \mathrm{LPS} \cdot \mathrm{R}-\mathrm{k}_{\mathrm{syn}} \cdot \mathrm{R} \\
& \frac{\mathrm{dmRNA}, \mathrm{R}}{\mathrm{dt}}=\mathrm{K}_{\mathrm{in}, \mathrm{mRNA}, \mathrm{R}} \cdot\left(1+\mathrm{H}_{\mathrm{mRNA}, \mathrm{DR}{ }^{*}}\right)-\mathrm{K}_{\text {out }, \mathrm{mRNA}, \mathrm{R}} \cdot \mathrm{mRNA}, \mathrm{R} \\
& \frac{\mathrm{d}(\mathrm{LPSR})}{\mathrm{dt}}=\mathrm{k}_{1} \cdot \mathrm{LPS} \cdot \mathrm{R}-\mathrm{k}_{3} \cdot(\mathrm{LPSR})-\mathrm{k}_{2}(\mathrm{LPSR}) \\
& \frac{\mathrm{dDR}^{*}}{\mathrm{dt}}=\mathrm{k}_{3} \cdot \frac{\mathrm{LPSR}}{\mathrm{A}}-\mathrm{k}_{4} \cdot \mathrm{DR}^{*}+\mathrm{k}_{\mathrm{c}} \cdot\left(\frac{\left[\mathrm{DR}^{*}\right]^{5}}{1+\left[\mathrm{DR}^{*}\right]^{5}}\right) \\
& \frac{\mathrm{dP}}{\mathrm{dt}}=\frac{\mathrm{K}_{\mathrm{in}, \mathrm{P}}}{\mathrm{A}} \cdot\left(1+\mathrm{H}_{\mathrm{P}, \mathrm{DR}}{ }^{*}\right) \cdot\left(1+\mathrm{H}_{\mathrm{P}, \mathrm{E}}\right)-\mathrm{K}_{\text {out }, \mathrm{P}} \cdot \mathrm{P} \\
& \frac{\mathrm{dA}}{\mathrm{dt}}=\mathrm{K}_{\mathrm{in}, \mathrm{A}} \cdot\left(1+\mathrm{H}_{\mathrm{A}, \mathrm{P}}\right) \cdot\left(1+\mathrm{H}_{\mathrm{A}, \mathrm{E}}\right)-\mathrm{K}_{\mathrm{out}, \mathrm{A}} \cdot \mathrm{A} \\
& \frac{\mathrm{dE}}{\mathrm{dt}}=\frac{\mathrm{K}_{\mathrm{in}, \mathrm{E}}}{\mathrm{A}} \cdot\left(1+\mathrm{H}_{\mathrm{E}, \mathrm{P}}\right)-\mathrm{K}_{\text {out, } \mathrm{E}} \cdot \mathrm{E} \\
& \mathrm{H}_{\mathrm{mRNA}_{\mathrm{R}}, \mathrm{DR}} \mathrm{k}_{\mathrm{mRNA}_{\mathrm{R}}, \mathrm{DR}} \cdot \mathrm{DR}^{*} \\
& \mathrm{H}_{\mathrm{P}, \mathrm{DR}}{ }^{*}=\mathrm{k}_{\mathrm{P}, \mathrm{DR}} * \mathrm{DR}^{*} \\
& \mathrm{H}_{\mathrm{P}, \mathrm{E}}=\mathrm{k}_{\mathrm{P}, \mathrm{E}} \cdot \mathrm{E} \\
& \mathrm{H}_{\mathrm{A}, \mathrm{P}}=\mathrm{k}_{\mathrm{A}, \mathrm{P}} \cdot \mathrm{P} \\
& \mathrm{H}_{\mathrm{A}, \mathrm{E}}=\mathrm{k}_{\mathrm{A}, \mathrm{E}} \cdot \mathrm{E} \\
& \mathrm{H}_{\mathrm{E}, \mathrm{P}}=\mathrm{k}_{\mathrm{E}, \mathrm{P}} \cdot \mathrm{P}
\end{aligned}
$$

Figure 5.2: Mathematical representation of an indirect response model of endotoxin-induced human inflammation. 
A self-limited inflammatory response to the endotoxin stimulus corresponds to resolved dynamic profiles for all the elements that constitute our model. In our computational model the host restores homeostasis without any external intervention. We speculate that the initial normalized concentration of the inflammatory stimulus (LPS) quickly decays so that it completely clears within the first 2 hours whereas the other essential components should return to their baseline within the first 24 hrs after the endotoxin administration (homeostasis) based on the design of the experiment. Given the available experimental data (Calvano, Xiao et al. 2005a) we can, in principle, evaluate appropriate model parameters using standard parameter estimation techniques. The relevant kinetic parameters are depicted in Table $\mathbf{5 . 1} \mathbf{1}^{2}$ whereas the performance of the model in reproducing the self-limited responses is shown in Figure 5.3.

Building a mathematical model that can predict relevant biological implications to the host response to endotoxin allows us to identify ways of both controlling and modulating such a complex phenomenon. Thus, the correctness of the model will be tested based on its ability to not only reproduce available data, but rather to qualitatively predict uncontrolled responses. Of particular interest are computational tests performed to predict the possibility and extent of abnormal responses resulting from various levels of inherent mechanistic dysregulation. In the following we will demonstrate the ability of our model to enable such "predictions" and provide further evidence of the appropriateness of the assumptions invoked in the development of the model.

\footnotetext{
${ }^{2}$ Estimated values of relevant model parameters are succinctly presented in Table 5.1 (see Appendix 5.2)
} 

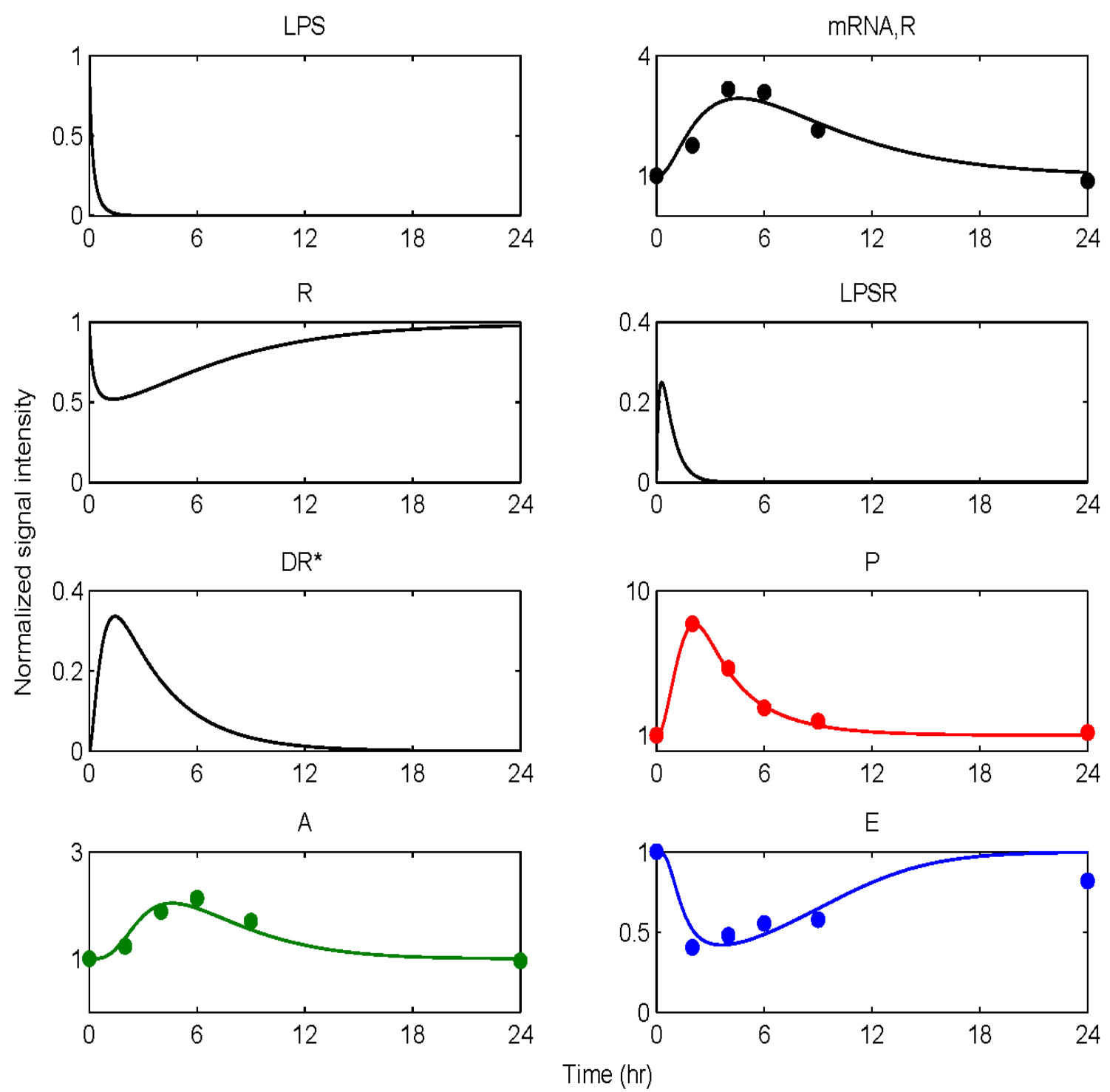

Figure 5.3: Model Building Results: Dynamic profiles of the elements that constitute the semi-mechanistic model of endotoxin-induced inflammation. Experimentally measured normalized mRNA transcript levels are denoted by symbols $(\bullet)$, solid lines $(-)$ are the model predictions. 


\subsubsection{Designining and performing in silico experiments}

We have notionally devised three levels of in silico "predictions". Regarding these "predictions" we should underline that such a term is loosely used to demonstrate the potential of our model to reproduce clinically relevant conditions. First, we explore the implication of increasing levels of initial insult since this would probably constitute the most obvious irreversible disturbance. Then we explore possible mechanistic dysregulation which may reflect secondary effects that lead to potential malfunction of the response leading to sustained inflammation. Finally, we explore the emergence of "memory" effects and evaluate the implication of priming the host with controlled level of endotoxin stimulus as a prequel to the main inflammatory stimulus.

\section{Implications of increased insult}

High concentrations of the inflammatory insult can be responsible for the amplification of the host immune response (Munford 2006), followed by a dysregulation in the host defense intrinsic dynamics leading to a an unconstrained inflammatory response even after the circulating levels of LPS have been cleared. In order to simulate such a scenario we increase the initial condition of LPS at various levels, i.e. $\operatorname{LPS}(\mathrm{t}=0 \mathrm{hr})=(1,2,3,4)$ inferring in silico the progression of the inflammatory trajectory, Figure 5.4. We observe that when the concentration of the inflammatory stimulus exceeds a critical threshold, the inflammatory response does not abate. In this case it is the host response to endotoxin rather than the stimulus itself that yields the progression of a systemic inflammatory response syndrome that fails to resolve. Clinically, in a retrospective analysis of critically ill patients (Reyes, Brimioulle et al. 1999), a progression of septic shock characterized a 
number of patients without documented infection. Namely, the mortality rate of these patients was higher compared to infected patients.

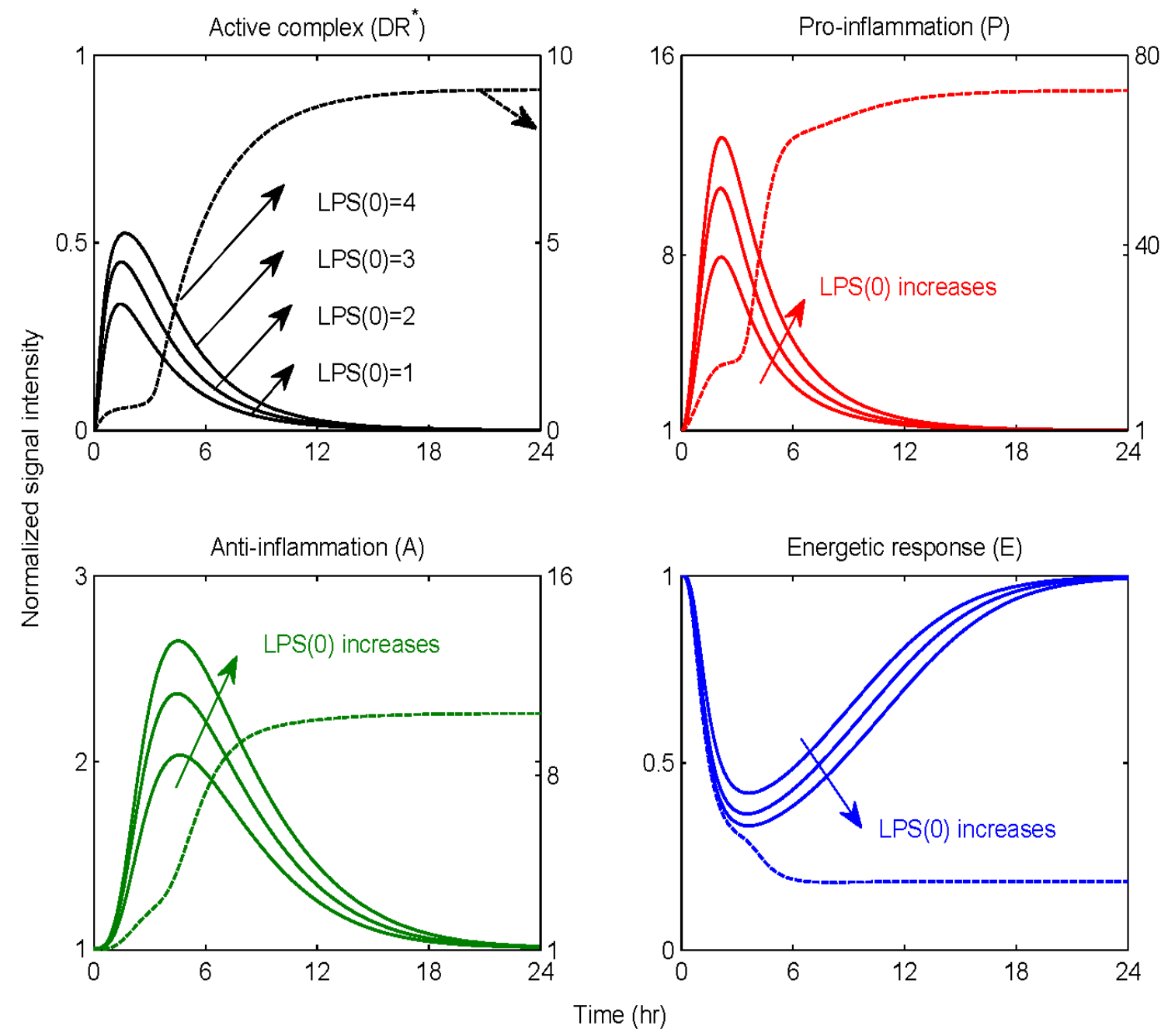

Figure 5.4: Temporal responses of critical inflammatory components for various initial conditions of the inflammatory stimulus. A high concentration of LPS can cause a malfunction in the dynamics of the host response to infection described by an exacerbated inflammatory response (dashed line). Solid lines correspond to self-limited responses, the dashed line represent a predicted unconstrained inflammatory response when the LPS concentration exceeds a critical value. 


\section{Modes of dysregulation of the inflammatory response}

The dynamics of the host response to an inflammatory stimulus are highly complex and non-linear, suggesting that a dysregulation in the dynamics of the inflammatory response in restoring homeostasis can have multiple causes. One such possibility is associated with a malfunction in the clearance rate of endotoxin (which corresponds to a higher exposure of the host response to the stimulus followed by persistence in the concentration of LPS). Secondly, a dysregulation in the intracellular dynamics can occur being responsible for an aberrant response, given that improper regulations of TLR4 signaling might also be involved in the inflammatory component of a disease like sepsis (Bhattacharjee and Akira 2006); such a mode of dysregulation separates the host response dynamics from the insulting agent. The model was probed by appropriately manipulating parameters that include: (i) a reduction in the first order degradation rate of LPS and (ii) a reduction in the degradation rate of the active signaling complex.

\section{Malfunction in the clearance rate of LPS}

An increased exposure of the host response to the inflammatory stimulus leading to a persistent disease is simulated in Figure 5.5. Such a case is simulated by manipulating (decreasing) the parameter associated with the degradation rate of LPS. Although decreased degradation of LPS is not associated with a distinct, defined clinical condition, it is possible that this phenomenon may exist. For example, it is known that triglyceriderich lipoproteins bind to LPS and that these complexes are cleared by binding to lipoprotein receptors. Furthermore, these receptors are abundant in the liver which clears $\sim 70 \%$ of lipoproteins from the circulation. Therefore, it can be postulated that patients 
with liver dysfunction may have impaired clearance of LPS. But, even without this speculation, another purpose for simulating "decreased LPS clearance" was to determine the response of the model to differing, but plausible, perturbations (Lauffenburger and Kennedy 1980).
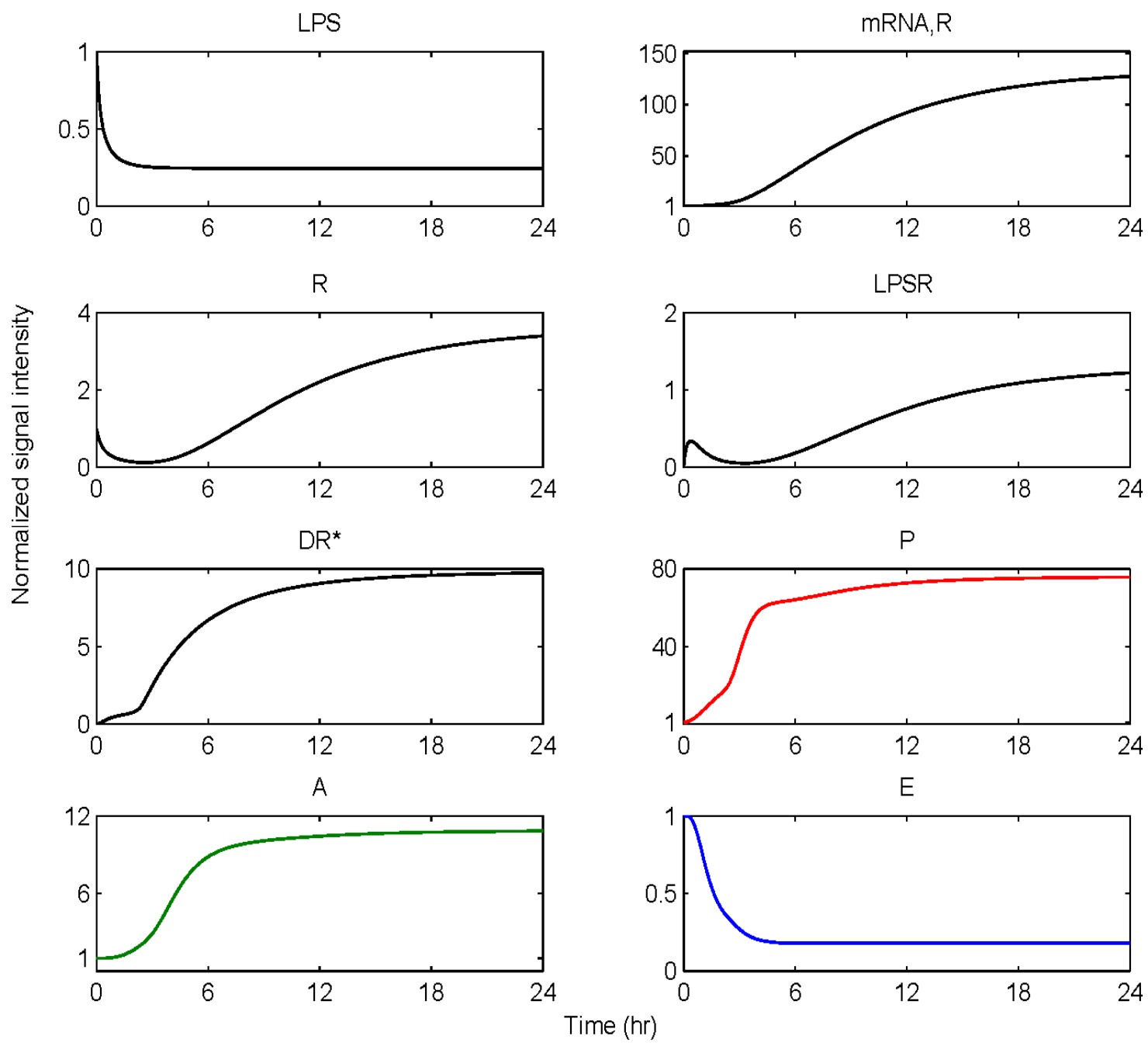

Figure 5.5: Temporal responses of inflammatory components in persistent infectious inflammatory response where the inflammatory stimulus cannot be eliminated responsible for the observed persistence in the dynamic profiles of the inflammatory constituents. Reducing the degradation rate of LPS to half of its initial value the inflammatory stimulus cannot be cleared. 
As seen in Figure 5.5 decreasing the degradation rate to half of its initial value (selflimited response) we observe that even small amounts of endotoxin might account for an overwhelming inflammatory response. Such persistence in the inflammatory stimulus leads to a sustained elevation of the activated intracellular signaling complex (DR*) which subsequently accounts for an uncompensated inflammatory response. In particular, there is an overexcitation of both pro- and anti- inflammatory mediators that are coupled with the uncontrolled regulation of the energetic response which settles to an uncontrolled state (energetic depletion). Such a simulated clinical scenario lies in agreement with experimental evidence about persistent endotoxin activity in critically ill patients undergoing gram-negative sepsis (Marshall, Walker et al. 2002).

\section{Maladaption in the active intracellular signaling $D R^{*}$}

It is now generally accepted that the host response plays a pivotal role in determining the outcome of an overwhelming inflammatory response. Thus, our model allows us to explore another mode of unconstrained inflammatory response that emerges from a dysregulation in the intracellular dynamics downstream of the ligand - receptor complex. In general, downstream of the activated LPSR complex, there are various kinases, second messengers that are being activated in response to LPS that are constituents of the signal transduction cascade. A dysregulation in the dynamics of the intracellular domain might hamper the homeostatic control of its domain, accounting for a persistent activated signaling that will over - excite the elementary inflammatory mediators, Figure 5.6. We explore such a perturbation in the dynamics of the system by decreasing the degradation rate of the active signaling complex $\left(\mathrm{DR}^{*}\right)$ to a value which is about one third of its initial value which corresponds to a self-limited inflammatory response. 

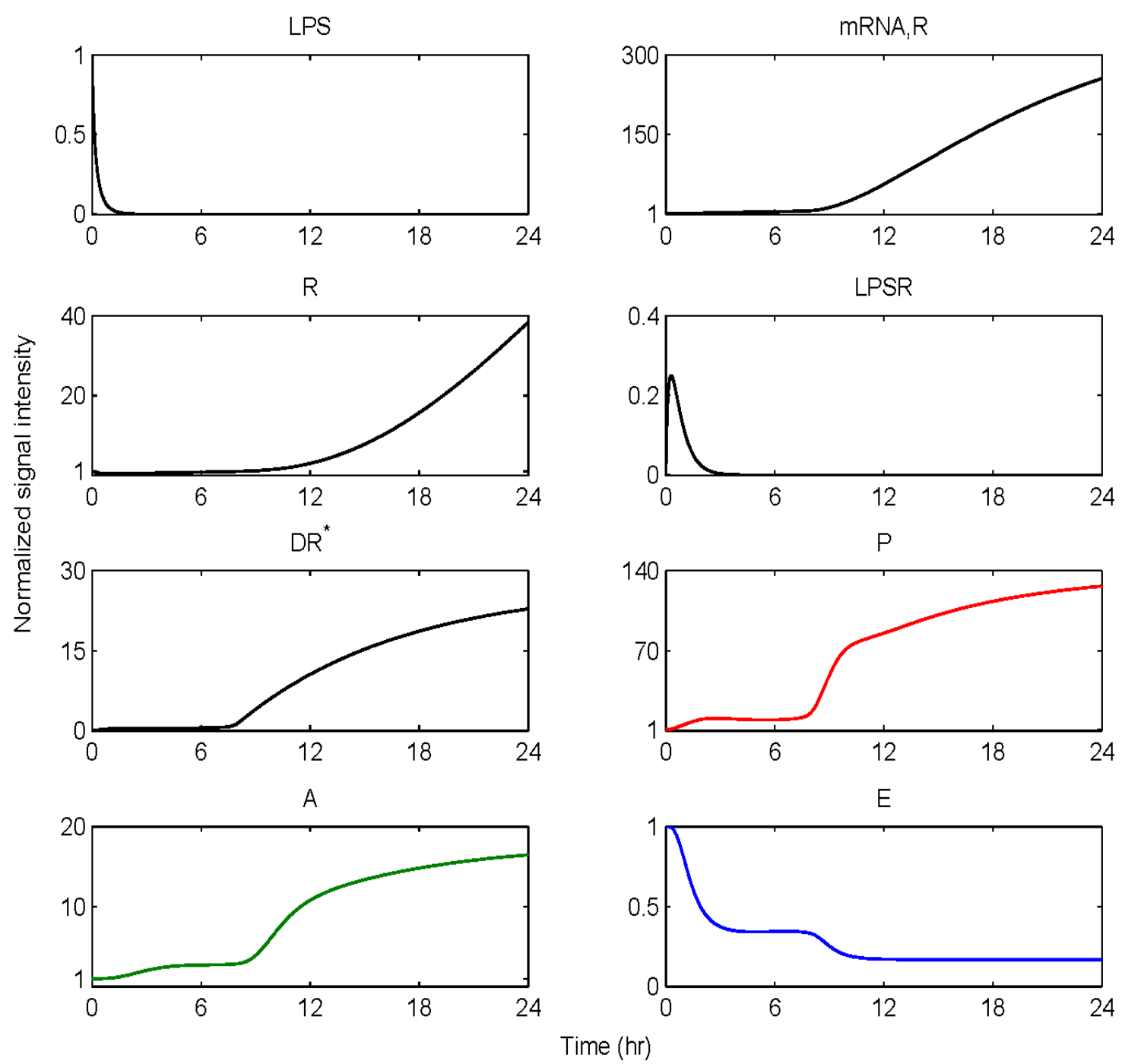

Figure 5.6: Temporal profiles of persistent non - infectious inflammation. It is not the infection itself but rather the host response to infection that plays a determinant role in controlling the outcome of an overwhelming inflammatory response. Manipulating the degradation rate of the activated intracellular signaling (i.e. reducing it by $1 / 3$ of its initial value) perturbs the homeostasis of the system.

The unconstrained inflammatory effect as shown in Figure 5.6 occurs downstream of the activated signaling complex and as result the rate of the formed complexes is not affected by this perturbation given that the inflammatory stimulus is completely 
eliminated from the system. However, a dysregulation in the intracellular homeostasis can lead to a cytokine "storm" that is followed by a global disturbance in the inflammatory control system followed by elevated/uncompensated anti - inflammatory signaling. If the gene transcript of the receptor is persistently elevated it will also lead to an overproduction of protein receptors; therefore, the baseline of total free receptors is dysregulated. Both cases correspond to biological scenarios exploring different modes of dysregulation in the inflammatory response which suggest that there is a critical time interval during which any therapeutic intervention may restore homeostasis. In the drug discovery area there is emphasis on discovering endogenous mediators that can modulate the inflammatory response but they are not clinically tractable in terms of a broader therapeutic time window (Sama, D'Amore et al. 2004); for example, one reason for the failure of anti - TNF treatment for sepsis in clinical settings may be due to its narrow therapeutic window (very early release).

\section{The emergence of memory effects}

\section{"Rapid" tolerance}

Repeated doses of endotoxin stimulus in many instances is considerably characterized by a less vigorous immune system which is namely known as endotoxin tolerance (Fan and Cook 2004). Even though the phenomenon of endotoxin tolerance involves the administration of low, repeated doses of endotoxin over periods of time ranging from one day to a week (Wysocka, Robertson et al. 2001),herein, we opt to investigate the response of the system being pre-exposed to low dose of LPS for less than a day. This is because in our proposed model all the interacting components do resolve within the first $24 \mathrm{hr}$ 
while the system has not acquired a reprogramming dynamic state. However, published studies (McCall, Grosso-Wilmoth et al. 1993; Poll 1996) report that "rapid" endotoxin tolerance can be induced when the system is pre-exposed to a low endotoxin challenge for between 3-6hr. Thus, we simulate such a scenario administering low dose of LPS i.e. $\operatorname{LPS}(\mathrm{t}=0 \mathrm{hr})=0.5$ followed at $\mathrm{t}=4 \mathrm{hr}$ by the main endotoxin insult, $\operatorname{LPS}(\mathrm{t}=4 \mathrm{hr})=1$.

When the system is pre-exposed to a lower inflammatory stimulus for about $4 \mathrm{hrs}$ before the main endotoxin challenge our model predicts a much less vigorous inflammatory response as seen in Figure 5.7. In particular such an event, which can be characterized either as a short - time attenuation effect or else rapid tolerance is experimentally observed by the decreased concentrations of various pro-inflammatory mediators i.e. TNF-a, IL1B in response to secondary ex vivo whole blood stimulation with LPS (McCall, Grosso-Wilmoth et al. 1993). In addition to this, in the experimental study (Poll 1996) concentrations of the particular pro - inflammatory mediator (TNF-a) were decreased profoundly ex vivo at $3 \mathrm{hr}-6 \mathrm{hr}$ after in vivo endotoxin administration. However, by $24 \mathrm{hrs}$ the endotoxin tolerance had completely resolved. Such preconditioning results in an attenuation of the inflammatory response characterized by a less vigorous intracellular signaling coupled with the decreased peak level of the proinflammatory response. 

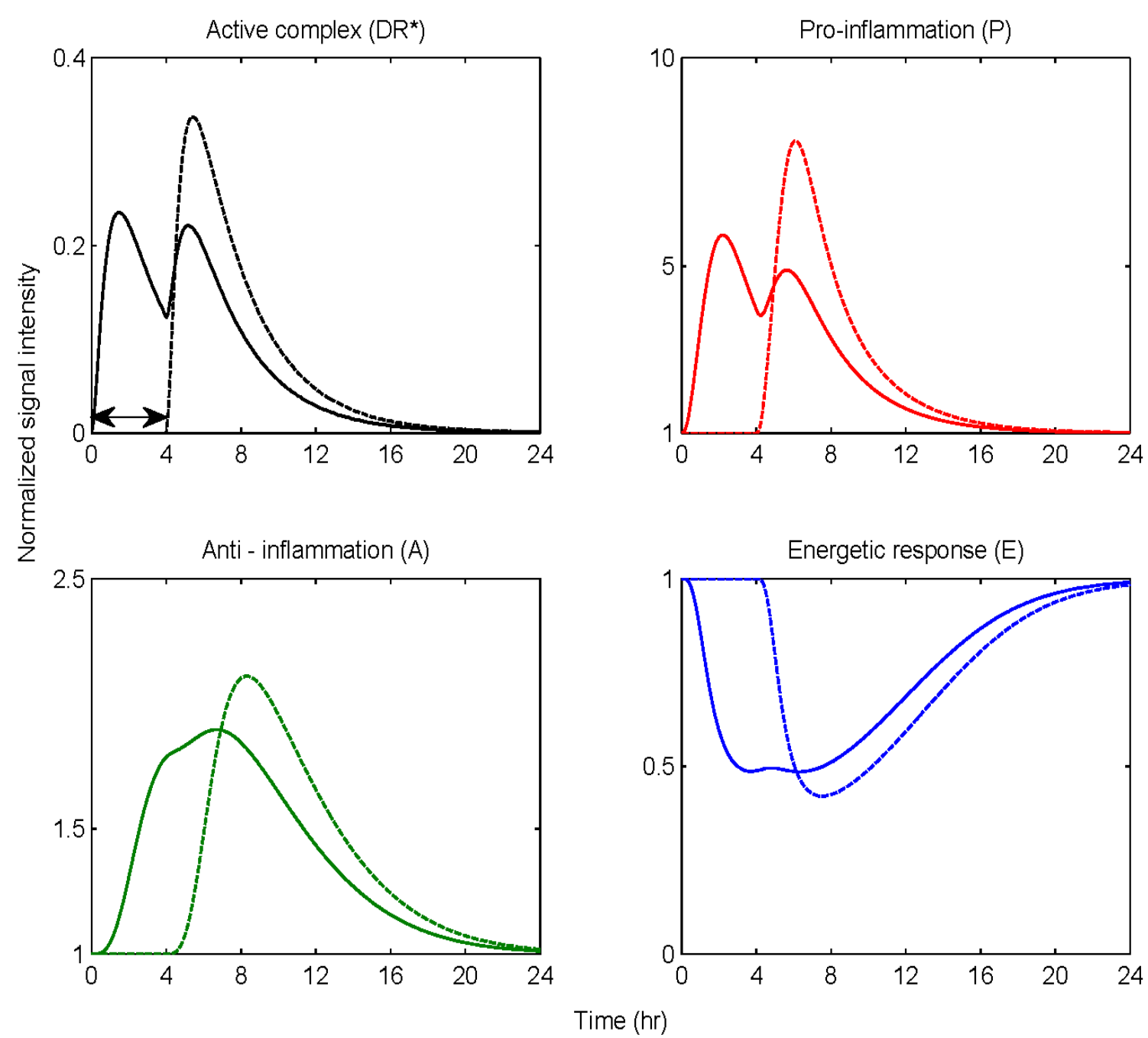

Figure 5.7: Rapid Endotoxin Tolerance: Pre - exposuring the system into a smaller inflammatory insult results in a reduction in the cell capacity to respond to the main endotoxin challenge which is characterized as a short-time attenuation scenario Solid line: $\operatorname{LPS}(\mathrm{t}=0 \mathrm{hr})=0.5 \& \operatorname{LPS}(\mathrm{t}=4 \mathrm{hr})=1$ Dashed line: $\operatorname{LPS}(\mathrm{t}=$ $0 \mathrm{hr})=0 \quad \& \operatorname{LPS}(\mathrm{t}=4 \mathrm{hr})=1$. 


\section{"Protective" tolerance}

The rapid tolerance scenario observed in Figure 5.7 implies that the emergence of controlled memory in the system, elicited after the first dose of endotoxin is administered, plays a major role in determining the modulated dynamics of the system in response to the second dose. If the system is primarily exposed to a much higher dose of endotoxin which is responsible for an overwhelming inflammatory response then the preexposure to lower non - lethal dose of LPS can modulate its intracellular dynamics that reverses the lethal outcome of the main endotoxin dose. This is a response characteristic of endotoxin hypo-responsiveness (or "protective" tolerance).

Protective" tolerance is another extended paradigm for endotoxin hyporesponsiveness that involves the administration of a sub-lethal dose of endotoxin followed by a high (lethal) one. Such pre-exposure "tolerates" the dynamics of the host reversing the implications of a high inflammatory insult as seen in Figure 5.8. Therefore, the magnitude of endotoxin doses plays a critical role in the underlying dynamics of the host and we reproduce such a scenario infusing low dose of LPS at $\mathrm{t}=0 \mathrm{hr}$, i.e., LPS $(\mathrm{t}=0 \mathrm{hr})=$ 1 followed by the administration of high endotoxin concentration, i.e., $\operatorname{LPS}(\mathrm{t}=4 \mathrm{hr})=4$. As a result the system shows a reduced capacity (hypo-responsiveness) in response to a high concentration of the inflammatory stimulus which may be associated with decreased TLR signaling by proteins that negatively regulate LPS-induced inflammatory responses (Cook, Pisetsky et al. 2004). 


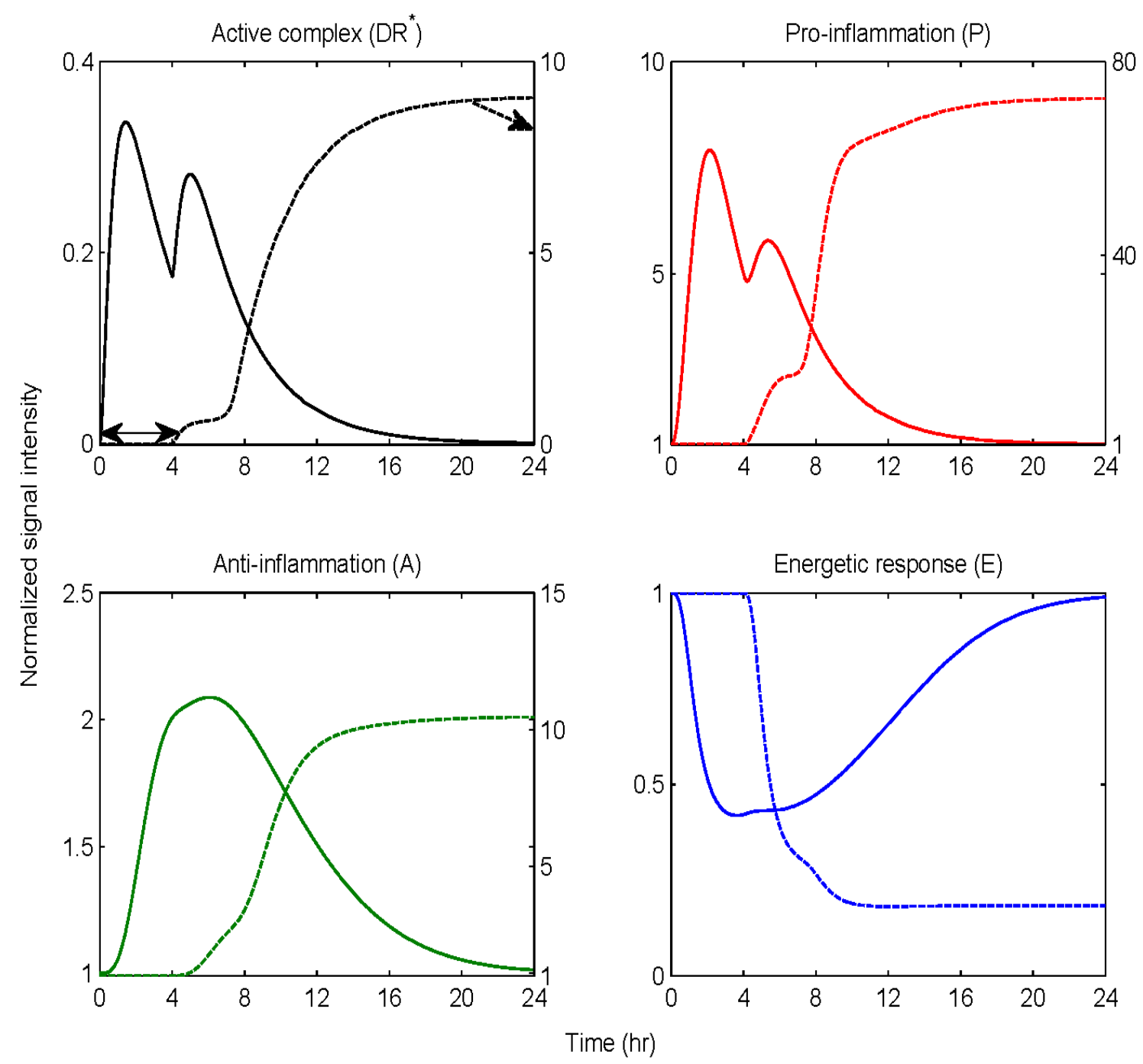

Figure 5.8: Endotoxin Hypo-responsiveness: Pre-existing infection might cause a profound hyporesponsiveness in system's response to a lethal LPS challenge Solid line: $\operatorname{LPS}(\mathrm{t}=0 \mathrm{hr})=1 \& \operatorname{LPS}(\mathrm{t}=4 \mathrm{hr})=$ 4 Dashed line: $\operatorname{LPS}(\mathrm{t}=0 \mathrm{hr})=0 \& \operatorname{LPS}(\mathrm{t}=4 \mathrm{hr})=4$. 


\section{"Lethal" potentiation}

Not only the magnitude but also the timing of repeated doses of endotoxin are key determinants for discriminating between endotoxin tolerance and potentiation. Rapid tolerance and endotoxin hypo-responsiveness (or else "protective" tolerance) are associated with an emergent acquired dynamic state of the system that actually modulates the response of the system not to respond rigorously to the primary endotoxin challenge. However, such an emergence is highly time dependent which implies that if the repeated doses are characterized by a very short time interval it is possible the dynamics of the system to result in an overwhelming inflammatory response. Therefore, the successive administration of two inflammatory insults that individually account for constrained ("self-limited") inflammatory responses might be determinant to the outcome of sepsis (unresolved inflammatory response). Such an event can occur because of the absence of a "protective" memory in the system so that the system has not elicited its regulatory mechanism to compensate for the cumulative result of two successive doses. Such an abrupt insult might dysregulate the dynamics of the host response to infection having a detrimental effect in the physiological state of the system.

From the modeling standpoint, we simulate such a case administering at $\mathrm{t}=0 \mathrm{hr}$ low dose of endotoxin, i.e. $\operatorname{LPS}(\mathrm{t}=0 \mathrm{hr})=1$ which is shortly followed at $\mathrm{t}=0.5 \mathrm{hr}$ by another "sub-lethal" insult, i.e. $\operatorname{LPS}(\mathrm{t}=0.5 \mathrm{hr})=2$. Thus, the successive administration of low doses of LPS as seen in Figure 5.9 may perturb system's homeostasis towards the progression of an unresolved inflammatory response. 


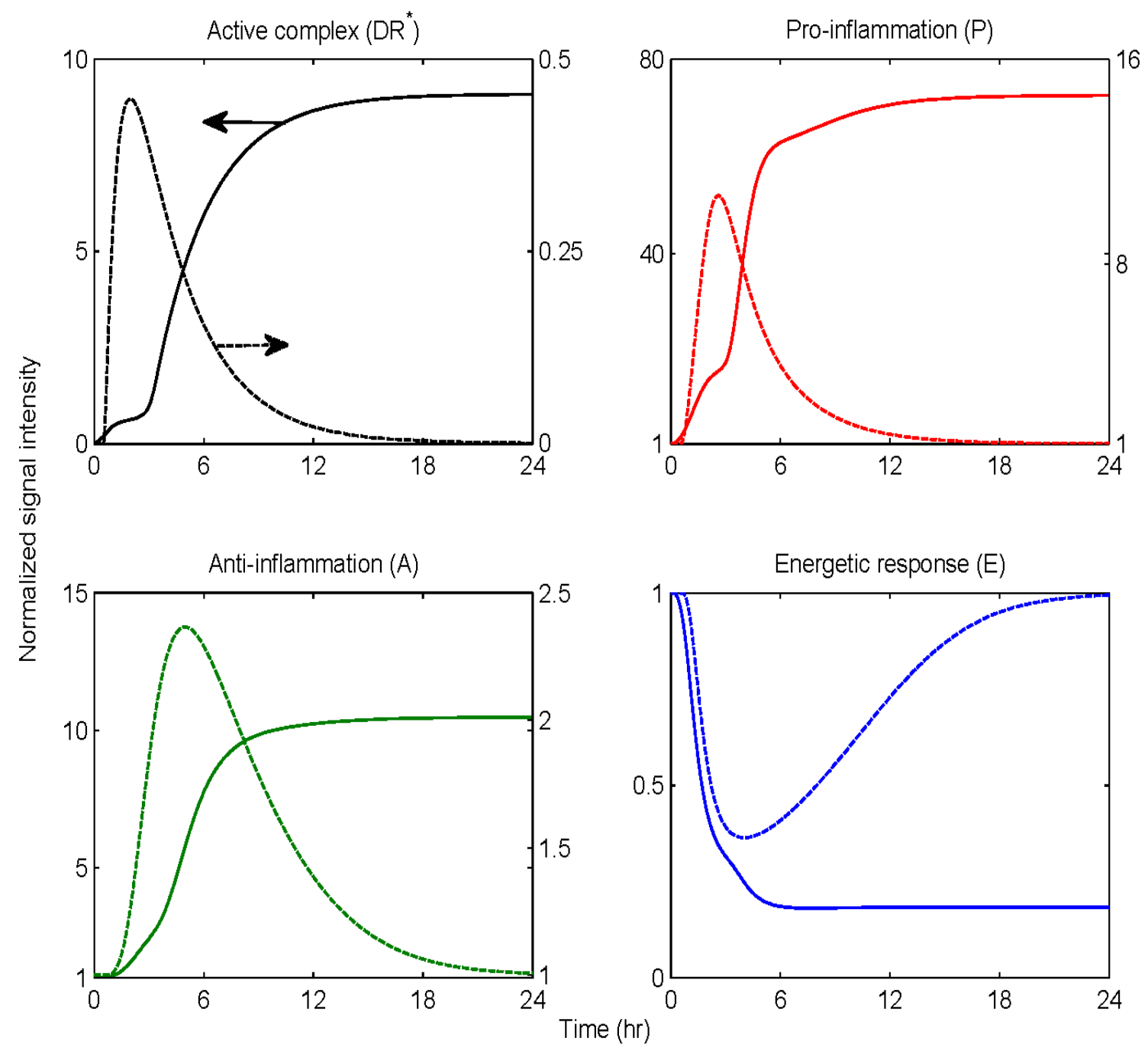

Figure 5.9: Lethal Potentiation: Successive administration of small doses of endotoxin can lead to an unresolved inflammatory response (due to loss of "regulatory" memory Solid line: $\operatorname{LPS}(\mathrm{t}=0 \mathrm{hr})=1 \&$ $\operatorname{LPS}(\mathrm{t}=0.5 \mathrm{hr})=2$ Dashed line: $\operatorname{LPS}(\mathrm{t}=0 \mathrm{hr})=0 \& \operatorname{LPS}(\mathrm{t}=0.5 \mathrm{hr})=2$. 
The aforementioned results indicate that the cellular response is critically affected by the mode of exposure, thus demonstrating the need for an appropriate, quantifiable, model to account for, and integrate the, various components constituting the response. Furthermore, our results clearly indicate that the dynamics of the response are definitely affected by the parameters defining the exposure to the inflammatory agent.

In conclusion, the proposed receptor mediated indirect response model of inflammation describes the sequence of inflammatory events connecting extracellular signals and transcriptional dynamics. The mechanistic-based indirect response model, allows us to identify possible critical targets either upstream of the activated signaling, such as endotoxin elimination rate, or downstream are associated with modulating the Toll- like receptor signaling pathway. The temporal profiles of the essential inflammatory components under an unresolved inflammatory state highlight the potential importance of early effective therapeutic interventions i.e. $(2 \mathrm{hr}-4 \mathrm{hr})$ whilst after $4 \mathrm{hrs}$ the system seems to have lost any potential for attenuation. Furthermore, we explored the possible effects of systemic perturbations associated with repeated pre-exposure to endotoxin (tolerance and potentiation scenarios) emphasizing timing and dosing as the key determinants for endotoxin hypo-responsiveness or lethality. Such a modeling approach enables us to gain a better understanding of the complexities of inflammation via the development of a more mechanistically interpretable model of human inflammation. 


\subsection{Appendix to the indirect response model}

\subsubsection{Table of inflammatory relevant mediators}

\begin{tabular}{|l|l|}
\hline Model component & Description \\
\hline LPS & $\begin{array}{l}\text { Inflammatory stimulus (lipopolysaccharide) derived from } \\
\text { gram-negative bacteria }\end{array}$ \\
\hline R & Endotoxin signaling receptor (TLR4) \\
\hline LPSR & Endotoxin-TLR4 complex \\
\hline mRNA,R & Gene transcript of endotoxin receptor \\
\hline DR ${ }^{*}$ & Activated signaling complex \\
\hline P & Transcriptional pro-inflammatory response \\
\hline A & Transcriptional anti-inflammatory response \\
\hline E & Transcriptional energetic response \\
\hline
\end{tabular}

\subsubsection{Estimated values of parameters}

Table 5.1: The 21 relevant model parameters based on self-limited response data. The values for $k_{1}$ and $k_{2}$ are taken from (Shin, Lee et al. 2007).

\begin{tabular}{|c|c|c|}
\hline $\mathrm{k}_{\mathrm{lps}, \mathrm{1}}=4.500$ & $\mathrm{k}_{3}=2.000$ & $\mathrm{~K}_{\mathrm{out}, \mathrm{P}}=2.428$ \\
\hline $\mathrm{k}_{\mathrm{lps}, 2}=6.790$ & $\mathrm{~K}_{\mathrm{in}, \mathrm{mRNA}, \mathrm{R}}=\mathrm{K}_{\mathrm{ou}, \mathrm{mRNA}, \mathrm{R}}=0.211$ & $\mathrm{k}_{\mathrm{A}, \mathrm{P}}=0.022$ \\
\hline $\mathrm{k}_{\mathrm{syn}}=0.020$ & $\mathrm{k}_{\mathrm{mRNAR}, \mathrm{DR}}{ }^{*}=13.467$ & $\mathrm{~K}_{\mathrm{in}, \mathrm{E}}=0.050$ \\
\hline $\mathrm{k}_{1}=3.000$ & $\mathrm{k}_{\mathrm{p}, \mathrm{E}}=25.191$ & $\mathrm{~K}_{\mathrm{out}, \mathrm{E}}=0.234$ \\
\hline $\mathrm{k}_{\mathrm{p}, \mathrm{DR}}=15.717$ & $\mathrm{k}_{4}=0.330$ & $\mathrm{~K}_{\mathrm{in}, \mathrm{A}}=0.256$ \\
\hline $\mathrm{k}_{\mathrm{E}, \mathrm{P}}=3.644$ & $\mathrm{k}_{\mathrm{c}}=3.000$ & $\mathrm{~K}_{\mathrm{out}, \mathrm{A}}=0.860$ \\
\hline $\mathrm{k}_{2}=0.040$ & $\mathrm{~K}_{\mathrm{in}, \mathrm{P}}=0.093$ & $\mathrm{k}_{\mathrm{A}, \mathrm{E}}=2.291$ \\
\hline
\end{tabular}




\subsubsection{Steady state equations}

\begin{tabular}{|c|c|}
\hline Eq. (4.3) & $\begin{aligned} \frac{\mathrm{dmRNA}, \mathrm{R}}{\mathrm{dt}}=0 & \rightarrow \mathrm{K}_{\mathrm{in}, \mathrm{mRNA}, \mathrm{R}} \cdot\left(1+\mathrm{k}_{\mathrm{mRNA}_{\mathrm{R}}, \mathrm{DR}^{*}} \cdot \mathrm{DR}_{\mathrm{ss}}^{*}\right)-\mathrm{K}_{\text {out,mRNA }, \mathrm{R}} \cdot \mathrm{mRNA}, \mathrm{R}_{\mathrm{ss}}=0 \\
\mathrm{~K}_{\text {out,mRNA }} & =\frac{\mathrm{K}_{\mathrm{in}, \mathrm{mRNAR} R} \cdot\left(1+\mathrm{k}_{\mathrm{mRNA}_{\mathrm{R}}, \mathrm{DR}^{*}} \cdot \mathrm{DR}^{*}(0)\right)}{\mathrm{mRNA}_{\mathrm{R}}(0)} \\
\mathrm{K}_{\text {out,mRNA }} & =\mathrm{K}_{\mathrm{in}, \mathrm{mRNAR} R}\end{aligned}$ \\
\hline Eq. (4.6) & $\begin{aligned} \frac{\mathrm{dP}}{\mathrm{dt}}= & 0 \rightarrow \frac{\mathrm{K}_{\mathrm{in}, \mathrm{P}}}{\mathrm{A}_{\mathrm{ss}}} \cdot\left(1+\mathrm{k}_{\mathrm{P}, \mathrm{DR}} \cdot \mathrm{DR}_{\mathrm{ss}}^{*}\right) \cdot\left(1+\mathrm{k}_{\mathrm{P}, \mathrm{E}} \cdot \mathrm{E}_{\mathrm{ss}}\right)-\mathrm{K}_{\mathrm{out}, \mathrm{P}} \cdot \mathrm{P}_{\mathrm{ss}}=0 \\
\mathrm{~K}_{\text {out }, \mathrm{P}}= & \frac{\mathrm{K}_{\mathrm{in}, \mathrm{P}} \cdot\left(1+\mathrm{k}_{\mathrm{P}, \mathrm{DR}} * \mathrm{DR}^{*}(0)\right) \cdot\left(1+\mathrm{k}_{\mathrm{P}, \mathrm{E}} \cdot \mathrm{E}(0)\right)}{\mathrm{A}(0) \cdot \mathrm{P}(0)} \\
\mathrm{K}_{\text {out }, \mathrm{P}}= & \mathrm{K}_{\mathrm{in}, \mathrm{P}} \cdot\left(1+\mathrm{k}_{\mathrm{P}, \mathrm{E}}\right)\end{aligned}$ \\
\hline Eq. (4.7) & $\begin{array}{c}\frac{\mathrm{dA}}{\mathrm{dt}}=0 \rightarrow \mathrm{K}_{\mathrm{in}, \mathrm{A}} \cdot\left(1+\mathrm{k}_{\mathrm{A}, \mathrm{P}} \cdot \mathrm{P}_{\mathrm{ss}}\right) \cdot\left(1+\mathrm{k}_{\mathrm{A}, \mathrm{E}} \cdot \mathrm{E}_{\mathrm{ss}}\right)-\mathrm{K}_{\mathrm{out}, \mathrm{A}} \cdot \mathrm{A}_{\mathrm{ss}}=0 \\
\mathrm{~K}_{\text {out }, \mathrm{A}}=\frac{\mathrm{K}_{\mathrm{in}, \mathrm{A}} \cdot\left(1+\mathrm{k}_{\mathrm{A}, \mathrm{P}} \cdot \mathrm{P}(0)\right) \cdot\left(1+\mathrm{k}_{\mathrm{A}, \mathrm{E}} \cdot \mathrm{E}(0)\right)}{\mathrm{A}(0)} \\
\mathrm{K}_{\text {out }, \mathrm{A}}=\mathrm{K}_{\mathrm{in}, \mathrm{A}} \cdot\left(1+\mathrm{k}_{\mathrm{A}, \mathrm{P}}\right) \cdot\left(1+\mathrm{k}_{\mathrm{A}, \mathrm{E}}\right)\end{array}$ \\
\hline Eq. (4.8) & $\begin{aligned} \frac{\mathrm{dE}}{\mathrm{dt}}= & 0 \rightarrow \frac{\mathrm{K}_{\mathrm{in}, \mathrm{E}}}{\mathrm{A}_{\mathrm{ss}}} \cdot\left(1+\mathrm{k}_{\mathrm{E}, \mathrm{P}} \cdot \mathrm{P}_{\mathrm{ss}}\right)-\mathrm{K}_{\mathrm{out}, \mathrm{E}} \cdot \mathrm{E}_{\mathrm{ss}}=0 \\
\mathrm{~K}_{\text {out }, \mathrm{E}} & =\frac{\mathrm{K}_{\mathrm{in}, \mathrm{E}} \cdot\left(1+\mathrm{k}_{\mathrm{E}, \mathrm{P}} \cdot \mathrm{P}(0)\right)}{\mathrm{A}(0) \cdot \mathrm{E}(0)} \rightarrow \mathrm{K}_{\text {out }, \mathrm{E}}=\mathrm{K}_{\mathrm{in}, \mathrm{E}} \cdot\left(1+\mathrm{k}_{\mathrm{E}, \mathrm{P}}\right)\end{aligned}$ \\
\hline
\end{tabular}

5.2.4 Initial conditions of relevant model components

\begin{tabular}{|l|l|l|l|}
\hline $\operatorname{LPS}(0)=1$ & $\mathrm{R}(0)=1$ & mRNA,R $(0)=1$ & $\operatorname{LPSR}(0)=1$ \\
\hline $\mathrm{DR}^{*}(0)=0$ & $\mathrm{P}(0)=1$ & $\mathrm{~A}(0)=1$ & $\mathrm{E}(0)=1$ \\
\hline
\end{tabular}




\subsection{An NF-kB dependent physicochemical model of systemic inflammation}

One of the key assumptions underpinning our modeling effort, as discussed in the previous section (Section 5.1), is that intracellular signaling cascades activating inflammation-specific transcriptional responses can be mathematically approximated by an aggregate variable, $\mathrm{DR}^{*}$, serving as a proxy of the activating signal. However, during the onset of an inflammatory response signaling pathways are activated for "translating" extracellular signals into intracellular responses (Aderem and Smith 2004). Such a signal transduction cascade converges to the activation of effector proteins (transcription factors) that regulate the expression of critical genes. Therefore, understanding more about the complex inflammatory reactions would require the development of computational models that incorporate biological information in the form of critical signaling cascades and kinetic rules. We wish therefore to deconvolute and interpret the combined activating signal with its "mechanistic" equivalence developing more interpretable and biologically relevant systems based models of inflammation.

The work to be discussed in this section aims to address the possibility of a semimechanistic host response model that integrates signaling and pharmacokinetic (PK) models of drug action for the modulation of the inflammatory response. Nuclear factor (NF)-kB is a central transcription factor that plays a major role in driving the inflammatory response (Senftleben 2003). Anti-inflammatory drugs such as corticosteroids play a critical role in modulating the progression of inflammation (van der Poll, Barber et al. 1996). We opt therefore to develop a physicochemical model of human inflammation that couples pro-inflammatory pathways with PK models of corticosteroids, to be used as a template for assessing anti-inflammatory intervention strategies. 


\subsubsection{Developing physicochemical models of acute inflammation in humans}

In order to introduce a finer level of detail in our computational model of inflammation we wish to deconvolute and interpret mechanistically the combined signal DR*. In the original model, $\mathrm{DR}^{*}$ represent the event activating the transcription of the proinflammatory response $(\mathrm{P})$ which in turn initiates the inflammatory response. As such, $\mathrm{DR}^{*}$ is the signal activating, i.e., transcriptionally regulating, the expression of the proinflammatory genes. Thus, the mechanistic equivalent of $\mathrm{DR}^{*}$ would be the signaling cascade that activates pro-inflammatory transcription factors controlling the expression of the pro-inflammatory genes as illustrated in Figure 5.10.

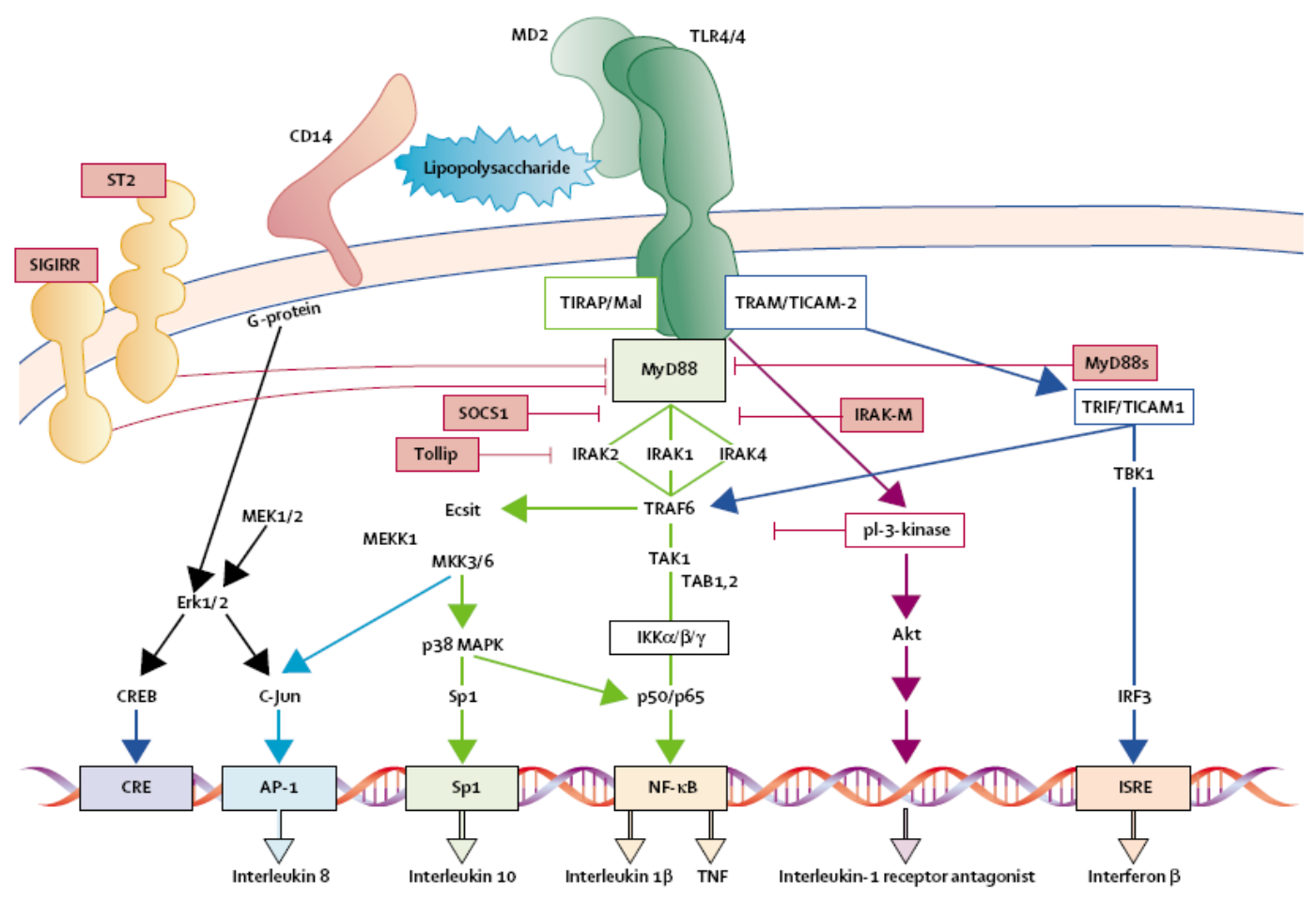

Figure 5.10: Endotoxin-induced intracellular signaling cascade. The inflammatory response is activated when endotoxin is recognized by pathogen recognition receptors that triggers downstream critical signaling modules for the activation of inflammatory relevant transcriptional factors (Annane, Bellissant et al. 2005). 
Although a large family of transcription factors is known to be involved in inflammation, we focus on a particular family, NF-kB, for two reasons. First, the nuclear factor $\mathrm{kB}$ family is known to be a major player in the inflammatory response (Saklatvala, Dean et al. 2003) and as such it has been widely studied as a major contributor. Second, the fact the NF-kB plays an important role has led to the development of numerous, independent, modeling approaches in order to quantify the expected response of its signaling cascade (Hoffmann, Levchenko et al. 2002b). Therefore, we introduce the NF$\mathrm{kB}$ signal transduction cascade as the prototypical module for initiating and controlling the expression of pro-inflammatory genes.

Numerous signaling molecules and reactions participate in the NF-kB signaling pathway (Hoffmann, Levchenko et al. 2002b). However, sensitivity analysis (Ihekwaba, Broomhead et al. 2004) demonstrated that the activity of NF-kB is maximally modulated by a reduced set of basis signaling molecules (IKK, IKBa and NF-kB). As such (Krishna, Jensen et al. 2006) proposed a minimal model of NF-kB that accounts for the propensity of oscillations in the dynamic behavior of NF-kB activity. However, instead of simulating the kinase activity as a constant parameter and incorporating saturation degradation rates as discussed in (Krishna, Jensen et al. 2006), we propose to model IKK as a transient signal. Qualitatively, the dynamic IKK activity corresponds to its intracellular concentration and it serves as the "input signal" for the subsequent activation of NF-kB signaling module, Eq. (5.15) - (5.17). 


$$
\begin{aligned}
& \frac{\mathrm{dIKK}}{\mathrm{dt}}=\mathrm{k}_{3} \cdot(\mathrm{LPSR}) /(1+\mathrm{IkBa})-\mathrm{k}_{4} \cdot \mathrm{IKK}+\mathrm{P} \cdot\left(\frac{\mathrm{IKK}^{2}}{1+\mathrm{IKK}^{2}}\right) \\
& \frac{\mathrm{dNFkB}}{\mathrm{dt}}=\frac{\mathrm{k}_{\mathrm{NFkB}, 1} \cdot \mathrm{IKK} \cdot\left(1-\mathrm{NFkB}_{\mathrm{n}}\right)}{(1+\mathrm{IkBa})}-\mathrm{k}_{\mathrm{NFkB}, 2} \cdot \mathrm{NFkB}_{\mathrm{n}} \cdot \mathrm{IkBa} \\
& \frac{\mathrm{dmRNA}_{\mathrm{IkBa}}}{\mathrm{dt}}=\mathrm{K}_{\mathrm{in}, \mathrm{IkBa}} \cdot\left(1+\mathrm{k}_{\mathrm{IkBa}, 1} \cdot \mathrm{NFkBn}\right)-\mathrm{K}_{\mathrm{out}, \mathrm{IkBa}} \cdot \mathrm{mRNA}_{\mathrm{IkBa}}
\end{aligned}
$$

Thus, the cellular surface complex (LPSR) induces the activation of kinase activity (IKK) with a rate $\mathrm{k}_{3}$, while being eliminated with a rate $\mathrm{k}_{4}$, Eq. (5.15). The non-linear function of Hill-type is an essential functional form in order to achieve a bistability response in the dynamics of the probed system (Kerschen, Fernandez et al. 2007; Lehmann, Freudenberg et al. 1987; Rifkind 1967; Tschaikowsky, Schmidt et al. 1998). Such a bistability is an essential characteristic of the non-linear dynamics of inflammation, as suggested by various animal studies (Kerschen, Fernandez et al. 2007; Lehmann, Freudenberg et al. 1987; Rifkind 1967; Tschaikowsky, Schmidt et al. 1998; Wang, Bloom et al. 1999). In chronic inflammatory diseases, several cytokines might be responsible for perpetuating and amplifying the inflammatory reaction through the critical node (IKK) (Barnes and Karin 1997). Therefore, we simulate such an interaction by the presence of a positive feedback loop in the kinetics of kinase (IKK) activity. Assuming that $\mathrm{NFkB}_{\mathrm{n}}$, Eq. (5.16) is the fraction of total NF-kB that is in the nucleus, the term $\left(1-\mathrm{NFkB}_{\mathrm{n}}\right)$ denotes the available free cytoplasmic concentration of $\mathrm{NF}-\mathrm{kB}$ and herein the nuclear concentration $\left(\mathrm{NFkB}_{\mathrm{n}}\right)$ and nuclear activity are used interchangeably. The import rate of cytoplasmic NF-kB into the nucleus depends on the availability of the free cytoplasmic concentration $\left(1-\mathrm{NFkB}_{\mathrm{n}}\right)$ stimulated by the kinase activity (IKK). However, its degradation rate depends on the presence of its primary inhibitor (IkBa), as 
it retrieves nuclear NF-kB by forming an inactive complex in the cytoplasmic region (Carmody and Chen 2007). The dynamics of the gene transcript of IKBa (mRNA $\mathrm{mkBa}_{\text {), }}$ Eq. (5.17), are characterized by a zero order production rate $\left(\mathrm{K}_{\mathrm{in}, \mathrm{IkBa}}\right)$ and a first order degradation rate $\left(\mathrm{K}_{\text {out,IkBa }}\right)$, which is stimulated by NF-kB (Barnes and Karin 1997).

The protein inhibitor IkBa, Eq. (5.18), is the product of translation of its gene transcript (mRNA, $\mathrm{IkBa}$ ) and it degrades at a rate $\mathrm{k}_{\mathrm{I}, 2}$, which is stimulated by the kinase activity (IKK). Based on the premise that $\mathrm{IkB}_{\mathrm{a}}$ forms a complex with the available cytoplasmic NF-kB, mathematically we expressed this as the product (1-NFkBn) IkBa. From the modeling point of view, in order to achieve a zero steady state for the protein inhibitor IkBa we need the additional negative term $-\mathrm{k}_{\mathrm{I}, 1}$.

$$
\frac{\mathrm{dIkBa}}{\mathrm{dt}}=\mathrm{k}_{\mathrm{I}, 1} \cdot \mathrm{mRNA}_{\mathrm{IkBa}}-\mathrm{k}_{\mathrm{I}, 2} \cdot(1+\mathrm{IKK}) \cdot\left(1-\mathrm{NFkB}_{\mathrm{n}}\right) \cdot \mathrm{IkBa}_{-\mathrm{I}, 1}
$$

The dynamics of the gene transcript of the endotoxin signaling receptor $(\mathrm{mRNA}, \mathrm{R})$ are described by a zero order production rate $\left(\mathrm{K}_{\mathrm{in}, \mathrm{mRNA}, \mathrm{R}}\right)$ and a first order degradation rate $\left(\mathrm{K}_{\text {out,mRNA,R }}\right)$, Eq. (5.19) - (5.20).

$$
\begin{gathered}
\frac{\mathrm{d}(\mathrm{mRNA}, \mathrm{R})}{\mathrm{dt}}=\mathrm{K}_{\mathrm{in}, \mathrm{mRNA}, \mathrm{R}} \cdot\left(1+\mathrm{H}_{\mathrm{mRNA}_{\mathrm{R}}, \mathrm{P}}\right)-\mathrm{K}_{\text {out,mRNA }, \mathrm{R}} \cdot \mathrm{mRNA}, \mathrm{R} \\
\mathrm{H}_{\mathrm{P}, \mathrm{R}}=\mathrm{k}_{\mathrm{mRNA}_{\mathrm{R}}, \mathrm{P}} \cdot \mathrm{P}
\end{gathered}
$$

We assume that the pro-inflammatory signaling indirectly stimulates the transcriptional activation of endotoxin receptor (TLR4); which quantitatively is expressed by the linear function $\left(\mathrm{H}_{\mathrm{P}, \mathrm{R}}\right)$, Eq. (5.20). Recently, there is research effort to elucidate the unknown mechanism that drives the regulation of TLR4 expression (Abreu, Arnold et al. 2002) and research findings (Mahony, Pham et al. 2008) support the potential role of proinflammatory cytokines to up-regulate the TLR expression. 
At the transcriptional response level, instead of assuming the active signaling complex, DR ${ }^{*}$ of Eq. (5.5) to manifest the effect of LPS on the cellular response level, herein we assume that the nuclear activity of $\mathrm{NF}-\mathrm{kB}\left(\mathrm{NFkB}_{\mathrm{n}}\right)$ serves as the "active signal" that indirectly stimulates the production rate of the essential pro-inflammatory response (P), Eq. (5.21) - (5.23).

$$
\begin{gathered}
\frac{\mathrm{dP}}{\mathrm{dt}}=\frac{\mathrm{K}_{\mathrm{in}, \mathrm{P}} \cdot\left(1+\mathrm{H}_{\mathrm{P}, \mathrm{NFkBn}}\right) \cdot\left(1+\mathrm{H}_{\mathrm{P}, \mathrm{E}}\right)}{\mathrm{A}}-\mathrm{K}_{\mathrm{out}, \mathrm{P}} \cdot \mathrm{P} \\
\mathrm{H}_{\mathrm{P}, \mathrm{NFkBn}}=\mathrm{k}_{\mathrm{P}, \mathrm{NFkB}_{\mathrm{n}}} \cdot \mathrm{NFkB}_{\mathrm{n}} \\
\mathrm{H}_{\mathrm{P}, \mathrm{E}}=\mathrm{k}_{\mathrm{P}, \mathrm{E}} \cdot \mathrm{E}
\end{gathered}
$$

Mathematically the stimulation of the nuclear activity $\mathrm{NFkB}_{\mathrm{n}}$ is expressed by the linear function $\left(\mathrm{H}_{\mathrm{P}, \mathrm{NFkBn}}\right)$, Eq. (5.22) and downstream of the pro-inflammatory response we preserve the structure of the elements that constitute the anti-inflammatory and the energetic response the same as shown in Eq. (5.1) - (5.14). For example, the energetic response variable will be responsible for more pronounced inflammation and therefore stimulates the pro-inflammatory response $\left(\mathrm{H}_{\mathrm{P}, \mathrm{E}}\right)$, Eq. (5.23). The anti-inflammatory signaling component is assumed to inhibit the production rate of the pro-inflammatory transcriptional signature, Eq. (5.21). The transcriptional dynamics of anti-inflammation (A) and the energetic response (E) are modeled on the same manner as discussed in Eq. (5.7) - (5.8)

Two major differences exist between the model in Eq. (5.1) - (5.14) and the proposed NF-kB dependent host response model, Eq. (5.15) - (5.23). First, the "translation" of the active signaling complex $\left(\mathrm{DR}^{*}\right)$ into biologically relevant signaling compartments; namely involving the activation of NF-kB signaling module. Such "translation" allows us 
to simulate the positive interaction between the pro-inflammation and the intracellular signaling (IKK). Second, in Eq. (5.5) the variable $\mathrm{DR}^{*}$ is assumed to be the convoluted signal that propagates the LPS signaling initiating the transcriptional synthesis of both the pro-inflammatory response $(\mathrm{P})$ and the mRNA of TLR4 $\left(\mathrm{mRNA}_{\mathrm{R}}\right)$. However, in the model Eq. (5.15) - (5.23) the elucidation of $\mathrm{DR}^{*}$ to the NF-kB activity limits the potential structure of the model. That is to say NF-kB is a pro-inflammatory transcription factor and it is not involved in the transcriptional regulation of the gene that encodes for the protein TLR4. On the other hand, based on literature evidence we support the potential role of pro-inflammatory signaling in mediating the transcriptional machinery of TLR4 $(\mathrm{mRN}, \mathrm{R})$ and we take it into consideration in the extended structure of the model. The proposed physicochemical host response model sheds insight on the interactions of the elements that constitute the inflammatory response. It offers us "realistic" handles on evaluating the effectiveness of various intervention strategies that modulate the intrinsic dynamics of the system opening areas amenable to the design of effective treatment schedules (Kumar, Chow et al. 2008). In the present study we aim at exploring in silico the pharmacodynamic effect of particular immunomodulatory agents - corticosteroids in modulating the progression of an unresolved inflammatory response

\subsubsection{Modeling corticosteroid interventions}

The progression of a disease involves the perturbation in the intrinsic dynamics of a system from its homeostasis (Post, Freijer et al. 2005). The presence of a disturbance (stimulus) initiates complex interaction of components at multiple scales (genetic, molecular, cellular level). The administration of a drug aims at modulating the progression of the disease by interfering with either individual molecules or signaling 
pathways. As such, we will explore means of modulating the activity of NF-kB through the use of corticosteroids. Developing mechanistic models of inflammation allows us to both characterize the non-linear inflammatory trajectory under various "what-if" scenarios and importantly to evaluate the effectiveness of drug-based treatment strategies that modulate the dynamics of the system. Integrating the cellular mechanism of drug action on disease progression models sheds insight on the better characterization of their pharmacodynamic effect against the disease status.

In this study, we consider corticosteroids as the means for controlling (modulating) the inflammatory state. One of the key aspects is the integration of the opposing effect of two crucial signaling pathways: one associated with the transcriptional dynamics that are elicited in response to endotoxin stimulus (LPS) and one related to the genomic signaling of exogenous corticosteroids. Such a modeling approach allows us to explore the pharmacodynamic effect of corticosteroids against inflammation exploring various modes of action.

Significant prior research efforts have attempted to elucidate the mechanisms driving corticosteroid activity (Almon, DuBois et al. 2002; Almon, Dubois et al. 2005; Almon, Lai et al. 2005; Almon, DuBois et al. 2007; DuBois, Xu et al. 1995; Jusko 1994; Sun, DuBois et al. 1998; Xu, Sun et al. 1995) Such studies simulate the pharmacogenomic effect of glucocorticoids at the transcriptional level taking their mechanistic (signaling) action into account (Jusko, DuBois et al. 2005; Ramakrishnan, DuBois et al. 2002b) and mathematically is expressed by Eq. (5.24) - (5.28) (Ramakrishnan, DuBois et al. 2002b). 


$$
\begin{gathered}
\mathrm{F}=\mathrm{C}_{1} \cdot \mathrm{e}^{-\mathrm{k}_{1} \mathrm{t}}+\mathrm{C}_{2} \cdot \mathrm{e}^{-\mathrm{k}_{2} \mathrm{t}} \\
\frac{\mathrm{dR}_{\mathrm{m}}}{\mathrm{dt}}=\mathrm{k}_{\mathrm{syn} \_\mathrm{Rm}} \cdot\left(1-\frac{\mathrm{FR}(\mathrm{N})}{\mathrm{IC}_{50 \_\mathrm{Rm}}+\mathrm{FR}(\mathrm{N})}\right)-\mathrm{k}_{\mathrm{deg}} \cdot \mathrm{R}_{\mathrm{m}} \\
\frac{\mathrm{dR}_{\mathrm{F}}}{\mathrm{dt}}=\mathrm{k}_{\mathrm{syn} \_\mathrm{R}} \cdot \mathrm{R}_{\mathrm{m}}+\mathrm{R}_{\mathrm{f}} \cdot \mathrm{k}_{\mathrm{re}} \cdot \mathrm{FR}(\mathrm{N})-\mathrm{k}_{\mathrm{on}} \cdot \mathrm{F} \cdot \mathrm{R}_{\mathrm{F}}-\mathrm{k}_{\mathrm{dgr} \_\mathrm{R}} \cdot \mathrm{R}_{\mathrm{F}} \\
\frac{\mathrm{dFR}}{\mathrm{dt}}=\mathrm{k}_{\mathrm{on}} \cdot \mathrm{F} \cdot \mathrm{R}_{\mathrm{F}}-\mathrm{k}_{\mathrm{T}} \cdot \mathrm{FR} \\
\frac{\mathrm{dFR}(\mathrm{N})}{\mathrm{dt}}=\mathrm{k}_{\mathrm{T}} \cdot \mathrm{FR}-\mathrm{k}_{\mathrm{re}} \cdot \mathrm{FR}(\mathrm{N})
\end{gathered}
$$

In essence, the corticosteroid intervention envelope consists of a set of elementary interactions that involve: (i) the binding of the corticosteroid drug (F) to its cytosolic receptor $\left(\mathrm{R}_{\mathrm{F}}\right)$, (ii) the subsequent formation of the drug-receptor complex (FR) (iii) the translocation of the cytosolic complex to the nucleus $(\mathrm{FR}(\mathrm{N}))$ that alters the transcriptional machinery activating or repressing numerous genes and finally (iv) the autoregulation of the gene transcript of the glucocorticoid receptor $\left(\mathrm{R}_{\mathrm{m}}\right)$.

In the original study (Ramakrishnan, DuBois et al. 2002b), the drug disposition is modeled, driven by the available experimental data, via a bi-exponential kinetic model, Eq. (5.24) and the plasma concentration of the drug (F) is mathematically expressed by a kinetic model with $\mathrm{C}_{\mathrm{i}}$ and $\mathrm{k}_{\mathrm{i}}$ to be the coefficients of intercepts and slopes (Ramakrishnan, DuBois et al. 2002b; Sun, DuBois et al. 1998). However, in our case study drug disposition can be simplified and described by either a mono-exponential kinetic model that quantifies the plasma concentration of the steroid $(F)$ under conditions of intravenous injection or via the time invariant parameter $\left(\mathrm{R}_{\text {in }}\right)$ and the elimination rate $\mathrm{k}_{\mathrm{el}}$ for the case of a constant infusion mode of administration, as follows: 


$$
\frac{\mathrm{dF}}{\mathrm{dt}}=\left\{\begin{array}{l}
\mathrm{R}_{\mathrm{in}}-\mathrm{k}_{\mathrm{el}} \cdot \mathrm{F}, \text { infusion } \\
-\mathrm{k}_{\mathrm{el}} \cdot \mathrm{F}, \text { injection }
\end{array}\right.
$$

The dynamics of the gene transcript of the corticosteroid drug $\left(\mathrm{R}_{\mathrm{m}}\right)$, Eq. (5.25), are characterized by a zero order production rate $\left(\mathrm{k}_{\mathrm{syn} \_\mathrm{Rm}}\right)$ and a first order degradation rate $\left(\mathrm{k}_{\mathrm{deg}}\right)$. The active drug-receptor complex, $\mathrm{FR}(\mathrm{N})$, exerts an inhibitory effect towards the mRNA of the glucocorticoid receptor. The parameter $\mathrm{IC}_{50 \_\mathrm{Rm}}$ denotes the concentration of the nuclear drug-receptor complex $F R(N)$ at which the synthesis rate of the receptor drops at $50 \%$ of its baseline value. The dynamics of the free cytosolic receptor density, $\mathrm{R}_{\mathrm{F}}$, is modeled in Eq. (5.26) where $\mathrm{k}_{\mathrm{syn} \_\mathrm{R}}$ is the synthesis rate of receptor that stems from its transcription, $r_{f}$ is the fraction of the drug that is recycled, $\mathrm{k}_{\mathrm{re}}$ is the parameter that shows the recycling of drug from the nucleus to the cytosol and $\mathrm{k}_{\mathrm{on}}$ is a parameter associated with the drug-receptor binding. In addition to this, $\mathrm{k}_{\mathrm{dgr}} \mathrm{R}$ is the degradation rate of the receptor $\left(R_{F}\right)$. The formed cytosolic complex (FR), Eq. (5.27), depends upon the binding interaction $\mathrm{k}_{\mathrm{on}}$ of the ligand $(\mathrm{F})$ with its receptor $\left(\mathrm{R}_{\mathrm{F}}\right)$ and on its translocation rate $\mathrm{k}_{\mathrm{T}}$ to the nucleus. Therefore, the translocation of the drug-receptor complex to the nucleus accounts for the nuclear receptor complex FR(N), Eq. (5.28), which is the active complex that mediates the transcriptional induction of various genes.

Effectively, in the study (Jin, Almon et al. 2003), equations (5.24) - (5.28) simulate in rat liver the effect of plasma concentration of a corticosteroid drug after a single intravenous administration of $50 \mathrm{mg} / \mathrm{kg}$. The model parameters are estimated based on available experimental data and the qualitative structure of the integrated inflammatory model with the active corticosteroid intervention envelope is presented in Figure 5.11. We observe that the interaction of the corticosteroid drug $(F)$ with its receptor $\left(\mathrm{R}_{\mathrm{F}}\right)$ mediates the activation of the nuclear drug-receptor complex $(\mathrm{FR}(\mathrm{N}))$. This complex 
serves as the "active signal" that induces transcriptional alterations suppressing the mRNA of the glucocorticoid receptor $\left(R_{m}\right)$ which drives downstream the reduced cytosolic receptor density.
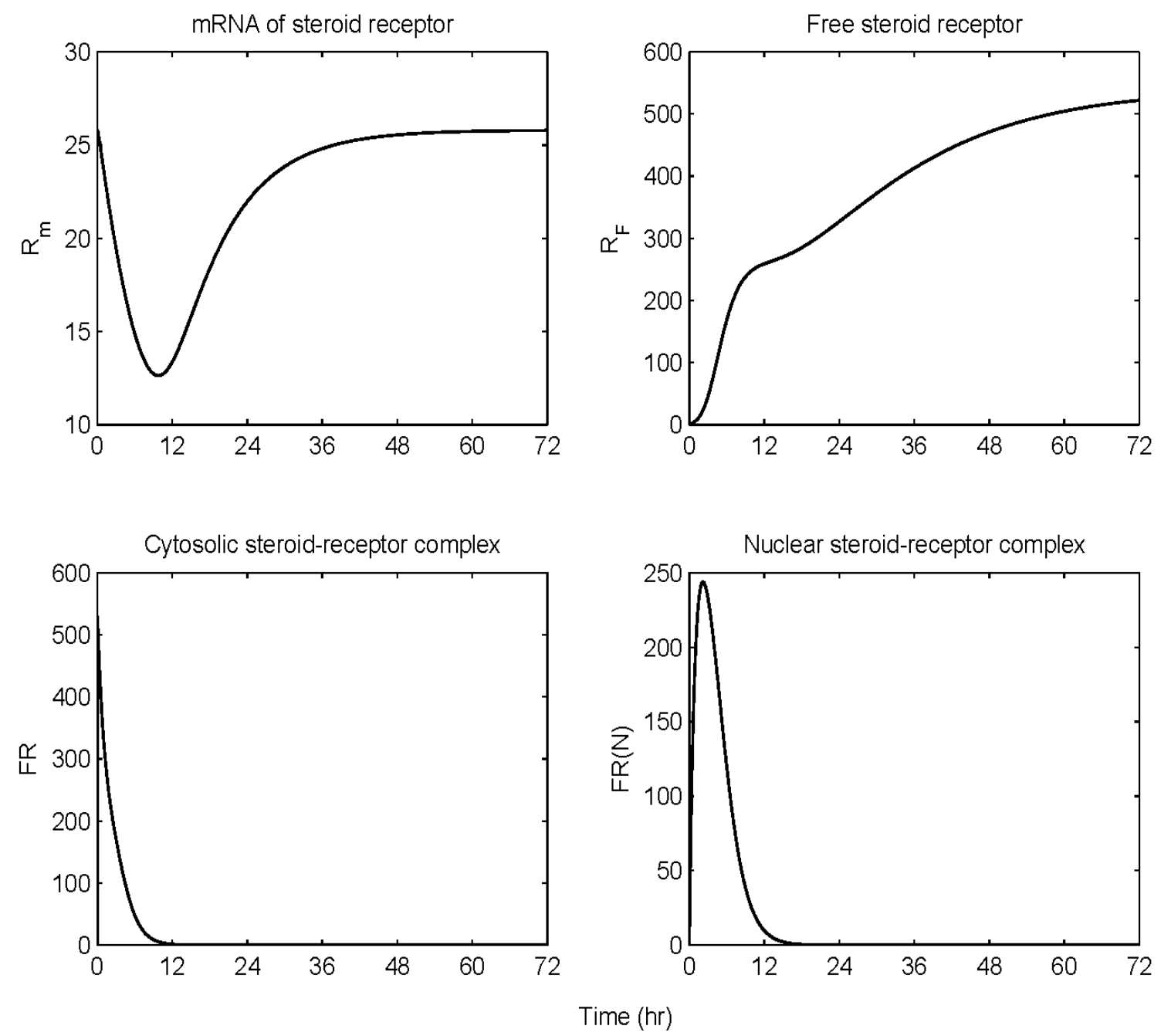

Figure 5.11: Dynamic evolution of model elements that constitute the mechanistic pharmacokinetic model of corticosteroids for a single intravenous administration (bolus injection) of $50 \mathrm{mg} / \mathrm{kg}$ and given the parameters and initial conditions extracted from (Jin, Almon et al. 2003).

Given, therefore, a quantification of the dynamics of corticosteroids and putative modes of action of CS in regulating the activity of NF-kB (Auphan, DiDonato et al. 
1995; Scheinman, Cogswell et al. 1995) we test the hypothesis that corticosteroids exert their immunosuppressive effect by enhancing the transcriptional synthesis of NF-kB's inhibitor IkBa (mRNA, $\left.{ }_{\mathrm{IkBa}}\right)$. Such a hypothesis does not imply that corticosteroids exert their anti-inflammatory mechanisms via only this mechanism. It has become increasingly evident (Rhen and Cidlowski 2005) that corticosteroids manifest their anti-inflammatory properties by various mechanisms that involve (i) either up-regulation of critical antiinflammatory proteins, i.e. IkBa, IL-10; (ii) or increased expression of an inhibitor to phospholipase $\mathrm{A}_{2}$ (annexin I) which subsequently leads to reduced formation of both arachidonic acid and platelet-activating factor as well as (ii) a disruption of the basal transcriptional machinery that inhibits the transcriptional activity of NF-kB. In this study, due to our inability to model all the mediators that may be affected by corticosteroids we opt to simulate the effect manifested by exogenous corticosteroids performing systematic perturbations on either the primary inhibitor of $\mathrm{NF}-\mathrm{kB}$, i.e. IkBa, as shown in Eq. (5.29) or the anti-inflammatory component of the response, Eq. (5.30).

$$
\begin{aligned}
& \frac{\mathrm{dmRNA}_{\mathrm{IkBa}}}{\mathrm{dt}}=\mathrm{K}_{\mathrm{in}, \mathrm{lkBa}} \cdot\left(1+\mathrm{k}_{\mathrm{IkBa}, 1} \cdot \mathrm{NFkB}_{\mathrm{n}}\right) \cdot\left(1+\mathrm{FR}(\mathrm{N})_{\mathrm{norm}}\right)-\mathrm{K}_{\mathrm{out}, \mathrm{lkBa}} \cdot \mathrm{mRNA}_{\mathrm{IkBa}} \\
& \frac{\mathrm{dA}}{\mathrm{dt}}=\mathrm{K}_{\mathrm{in}, \mathrm{A}} \cdot\left(1+\mathrm{H}_{\mathrm{A}, \mathrm{P}}\right) \cdot\left(1+\mathrm{H}_{\mathrm{A}, \mathrm{E}}\right) \cdot\left(1+\mathrm{FR}(\mathrm{N})_{\mathrm{norm}}\right)-\mathrm{K}_{\mathrm{out}, \mathrm{A}} \cdot \mathrm{A}
\end{aligned}
$$

where $\mathrm{FR}(\mathrm{N})_{\text {norm }}$ represents the normalized $\mathrm{FR}(\mathrm{N})$ signal that numerically it ranges between $(0,1)$ for a given drug dose. The reason for such normalization stems from the fact that the aim of this study is to provide a qualitative understanding about how the dynamics of a host undergoing an inflammatory response are modulated due to the corticosteroid intervention envelope. The mathematical representation of the integrated cellular host response model is succinctly presented in Eq. (5.31) - (5.36). 
LPS kinetics $\left\{\frac{\mathrm{dLPS}}{\mathrm{dt}}=\mathrm{k}_{\mathrm{lps}, 1} \cdot\right.$ LPS $\cdot(1-\mathrm{LPS})-\mathrm{k}_{\mathrm{lps}, 2} \cdot \mathrm{LPS}$

Ligand/receptor interactions $\left\{\begin{array}{l}\frac{\mathrm{dR}}{\mathrm{dt}}=\mathrm{k}_{\mathrm{syn}} \cdot \mathrm{mRNA}, \mathrm{R}+\mathrm{k}_{2} \cdot(\mathrm{LPSR})-\mathrm{k}_{1} \cdot \mathrm{LPS} \cdot \mathrm{R}-\mathrm{k}_{\mathrm{syn}} \cdot \mathrm{R} \\ \frac{\mathrm{d}(\mathrm{LPSR})}{\mathrm{dt}}=\mathrm{k}_{1} \cdot \mathrm{LPS} \cdot \mathrm{R}-\mathrm{k}_{3} \cdot(\mathrm{LPSR})-\mathrm{k}_{2} \cdot(\mathrm{LPSR}) \\ \frac{\mathrm{d}(\mathrm{mRNA}, \mathrm{R})}{\mathrm{dt}}=\mathrm{K}_{\mathrm{in}, \mathrm{mRNA}, \mathrm{R}} \cdot\left(1+\mathrm{H}_{\mathrm{mRNA}_{\mathrm{R}, \mathrm{P}}}\right) \\ -\mathrm{K}_{\text {out }, \mathrm{mRNA}, \mathrm{R}} \cdot \mathrm{mRNA}, \mathrm{R}\end{array}\right.$

NF-kB signaling $\left\{\begin{array}{l}\frac{\mathrm{dIKK}}{\mathrm{dt}}=\mathrm{k}_{3} \cdot(\mathrm{LPSR}) /(1+\mathrm{IkBa})-\mathrm{k}_{4} \cdot \mathrm{IKK}+\mathrm{P} \cdot\left(\frac{\mathrm{IKK}^{2}}{1+\mathrm{IKK}^{2}}\right) \\ \frac{\mathrm{dmRNB}_{\mathrm{It}}}{\mathrm{dt}}=\frac{\mathrm{k}_{\mathrm{NFkB}, 1} \cdot \mathrm{IKK} \cdot\left(1-\mathrm{NFkB}_{\mathrm{n}}\right)}{(1+\mathrm{IkBa})}-\mathrm{k}_{\mathrm{NFkB}, 2} \cdot \mathrm{NFkB}_{\mathrm{n}} \cdot \mathrm{IkBa} \\ \frac{\mathrm{dIkBa}}{\mathrm{dt}}=\mathrm{k}_{\mathrm{I}, 1} \cdot \mathrm{mRNA}_{\mathrm{IkBa}}-\mathrm{k}_{\mathrm{I}, 2} \cdot(1+\mathrm{IKK}) \cdot\left(1-\mathrm{NFkB}_{\mathrm{IkBa}, 1} \cdot \mathrm{NFkB}_{\mathrm{n}}\right)-\mathrm{K}_{\mathrm{out}, \mathrm{IkBa}} \cdot \mathrm{mRBa}_{\mathrm{IkBa}}\end{array}\right.$

Intrinsic transcriptional responses $\left\{\begin{array}{l}\frac{\mathrm{dP}}{\mathrm{dt}}=\mathrm{K}_{\mathrm{in}, \mathrm{P}} \cdot\left(1+\mathrm{H}_{\mathrm{P}, \mathrm{NFkBn}}\right) \cdot\left(1+\mathrm{H}_{\mathrm{P}, \mathrm{E}}\right) / \mathrm{A}-\mathrm{K}_{\mathrm{out}, \mathrm{P}} \cdot \mathrm{P} \\ \frac{\mathrm{dA}}{\mathrm{dt}}=\mathrm{K}_{\mathrm{in}, \mathrm{A}} \cdot\left(1+\mathrm{H}_{\mathrm{A}, \mathrm{P}}\right) \cdot\left(1+\mathrm{H}_{\mathrm{A}, \mathrm{E}}\right)-\mathrm{K}_{\mathrm{out}, \mathrm{A}} \cdot \mathrm{A} \\ \frac{\mathrm{dE}}{\mathrm{dt}}=\mathrm{K}_{\mathrm{in}, \mathrm{E}} \cdot\left(1+\mathrm{H}_{\mathrm{E}, \mathrm{P}}\right) / \mathrm{A}-\mathrm{K}_{\mathrm{out}, \mathrm{E}} \cdot \mathrm{E}\end{array}\right.$ 
Cortisol pharmacokinetics $\left\{\begin{array}{l}\frac{\mathrm{dF}}{\mathrm{dt}}=\left\{\begin{array}{l}\mathrm{R}_{\mathrm{in}}-\mathrm{k}_{\mathrm{el}} \cdot \mathrm{F}, \text { infusion } \\ -\mathrm{k}_{\mathrm{el}} \cdot \mathrm{F}, \text { injection }\end{array}\right. \\ \frac{\mathrm{dR} \mathrm{m}_{\mathrm{F}}}{\mathrm{dt}}=\mathrm{k}_{\mathrm{syn}_{-} \mathrm{Rm}} \cdot\left(1-\frac{\mathrm{FR}(\mathrm{N})}{\mathrm{I}_{50 \_\mathrm{Rm}}+\mathrm{FR}(\mathrm{N})}\right)-\mathrm{k}_{\mathrm{deg}} \cdot \mathrm{R}_{\mathrm{m}} \cdot \mathrm{R} \\ \frac{\mathrm{dF}}{\mathrm{dt}}=\mathrm{R}_{\mathrm{m}}+\mathrm{r}_{\mathrm{f}} \cdot \mathrm{k}_{\mathrm{re}} \cdot \mathrm{FR}(\mathrm{N})-\mathrm{k}_{\mathrm{on}} \cdot \mathrm{F} \cdot \mathrm{R}_{\mathrm{F}}-\mathrm{R}_{\mathrm{F}}-\mathrm{FR} \mathrm{k}_{\mathrm{dgr}_{-} \mathrm{R}} \cdot \mathrm{R}_{\mathrm{F}} \\ \frac{\mathrm{dFR}(\mathrm{N})}{\mathrm{dt}}=\mathrm{k}_{\mathrm{T}} \cdot \mathrm{FR}-\mathrm{k}_{\mathrm{re}} \cdot \mathrm{FR}(\mathrm{N})\end{array}\right.$

Cortisol pharmacodynamics $\left\{\begin{array}{l}\frac{\mathrm{dmRNA}_{\mathrm{IkBa}}}{\mathrm{dt}}=\mathrm{K}_{\mathrm{in}, \mathrm{IkBa}} \cdot\left(1+\mathrm{k}_{\mathrm{IkBa}, 1} \cdot \mathrm{NFkBn}_{\mathrm{n}}\right) \cdot\left(1+\mathrm{FR}(\mathrm{N})_{\mathrm{norm}}\right) \\ -\mathrm{K}_{\mathrm{out}, \mathrm{IkBa}} \cdot \mathrm{mRNA}_{\mathrm{IkBa}} \\ \frac{\mathrm{dA}}{\mathrm{dt}}=\mathrm{K}_{\mathrm{in}, \mathrm{A}} \cdot\left(1+\mathrm{H}_{\mathrm{A}, \mathrm{P}}\right) \cdot\left(1+\mathrm{H}_{\mathrm{A}, \mathrm{E}}\right) \cdot\left(1+\mathrm{FR}(\mathrm{N})_{\mathrm{norm}}\right) \\ -\mathrm{K}_{\mathrm{out}, \mathrm{A}} \cdot \mathrm{A}\end{array}\right.$

\subsubsection{Qualitative assessment of NF-kB dependent physicochemical host response} model

We have previously demonstrated that the transcriptional dynamics of human leukocytes exposed to bacterial endotoxin can be decomposed into to three elementary comprehensive responses (Foteinou, Calvano et al. 2007; Foteinou, Calvano et al. 2009a). Unlike previous approaches that concentrate on specific biomarkers, these elementary responses capture the functional dynamics and were shown to be related to proinflammatory (P), anti-inflammatory (A) and energetic (E) transcriptional events associated with the overall host response. The response is triggered by the activation of the NF-kB signaling module as a result of the formation of an activating signal associated 
with the binding of LPS to appropriate receptors. We hypothesize that NF-kB serves as a proxy for the inflammation specific transcription factors that initiates the expression of pro-inflammatory genes while its activity is primarily modulated by the kinase activity (IKK) and the inhibitor (IKBa). In this study, we seek to describe the host response to endotoxin via interacting modules that involve the propagation of LPS signaling on the transcriptional response level through NF-kB dependent mechanism and the genomic signaling of exogenous corticosteroids, as the putative controllers of inflammation. The corticosteroid intervention envelope consists of a set of elementary interactions that involve: (i) the binding of the corticosteroid drug $(F)$ to its cytosolic receptor $\left(R_{F}\right)$, (ii) the subsequent formation of the drug-receptor complex (FR) (iii) the translocation of the cytosolic complex to the nucleus $(\mathrm{FR}(\mathrm{N}))$ that alters the transcriptional machinery activating or repressing numerous genes and finally (iv) the autoregulation of the gene transcript of the glucocorticoid receptor $\left(\mathrm{R}_{\mathrm{m}}\right)$. All the interacting components and modules that constitute the NF-kB dependent physicochemical model of inflammation are shown in Figure 5.12. 


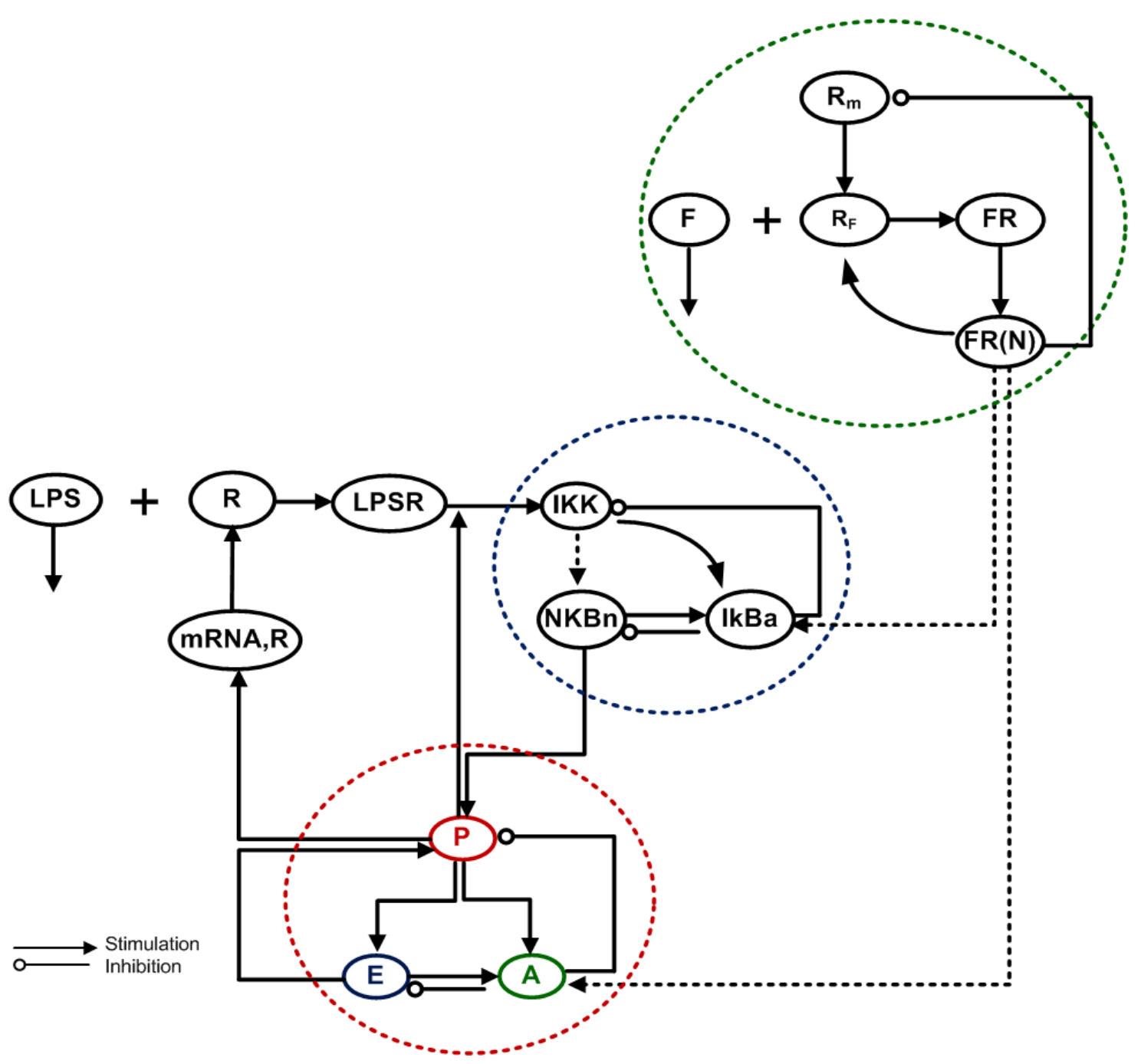

Figure 5.12: Schematic illustration of a reverse engineered model of systemic inflammation. Interacting modules involve the propagation of LPS signaling on the transcriptional response level coupled with the anti-inflammatory effect of corticosteroids. The propagation of LPS signaling involves the interaction of the inflammatory stimulus, LPS with its receptor (R) forming the surface complex (LPSR) which activates IKK activity. The IKK-dependent signal activates the translocation of NF-kB $\left(\mathrm{NFkB}_{\mathrm{n}}\right)$ through phosphorylation and degradation of its primary inhibitor, IkBa. The nuclear NF-kB (NFkBn) is autoregulated by its inhibitor protein, IKBa and stimulates the production rate of the pro-inflammatory response (P) while there is certain connectivity among the essential transcriptional signatures $(\mathrm{P}, \mathrm{A}, \mathrm{E})$. The mRNA 
of the receptor $\left(\mathrm{mRNA}_{\mathrm{R}}\right)$ is stimulated by pro-inflammation $(\mathrm{P})$ and it is translated to the surface protein (R). The corticosteroid intervention envelope consists of the corticosteroid drug (F) which binds to its intracellular receptor $\left(\mathrm{R}_{\mathrm{F}}\right)$ forming the cytosolic complex $(\mathrm{FR})$ that translocates to the nucleus $(\mathrm{FR}(\mathrm{N}))$ and modulates the dynamics of inflammation via an upregulation of anti-inflammatory proteins (IkBa, A) represented by dotted lines.

Kinetic parameters are estimated in order to best reproduce the essential transcriptional responses associated with experimental measurements, Table $\mathbf{5 . 2}^{3}$. The reconstructed dynamic profiles associated with a self-limited inflammatory response triggered by the activation of NF-kB signaling pathway are shown in Figure 5.13. In essence, a selflimited inflammatory response involves the successful elimination of the inflammatory stimulus within the first $2 \mathrm{hr}$ post-endotoxin administration while followed by a subsequent resolution within $24 \mathrm{hr}$.

\footnotetext{
${ }^{3}$ Estimated values of relevant model parameters are presented in Table 5.2 (see Appendix 5.4)
} 

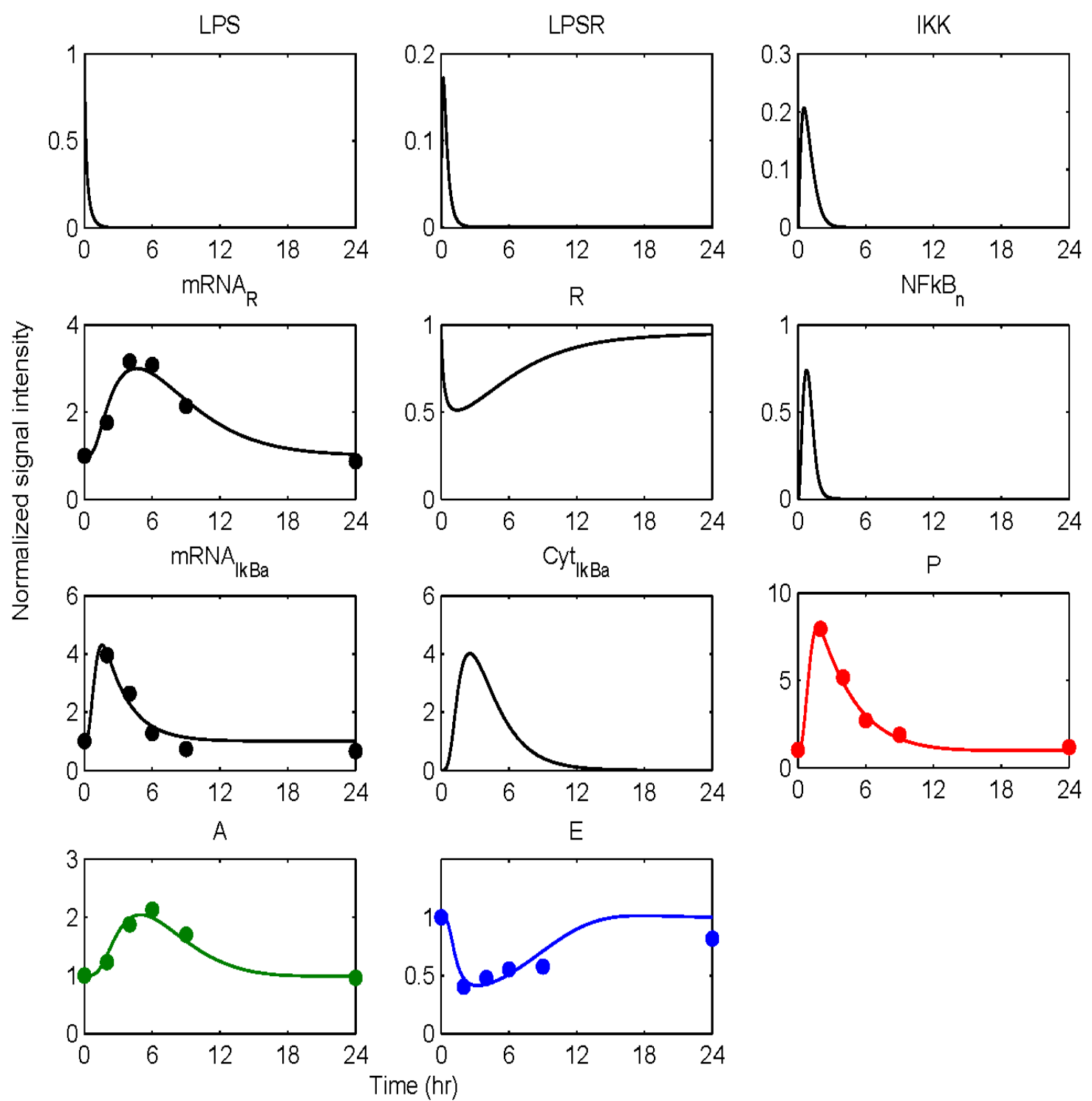

Figure 5.13: Estimation of relevant model parameters. Temporal profiles of the elements that constitute the NF-kB dependent model of endotoxin-induced inflammation. Solid lines $(-)$ correspond to model predictions whilst the symbols $(\bullet)$ denote for the experimentally measured transcriptional signatures. 


\section{$\underline{\text { Malfunction in LPS's clearance rate }}$}

The dynamics of the inflammatory response are highly complex such that a maladaption in the homeostasis of the system can be attributed to various reasons. One such possibility is associated with a malfunction in endotoxin clearance rate which corresponds to a higher exposure of the host to the stimulus. Under such conditions the inflammatory stimulus persists and leads to an aberrant NF-kB activity that drives downstream a chronic inflammatory response, Figure 5.14. Specifically, the inferred NF$\mathrm{kB}$ activity can be characterized as a "two-wave" response; initially it increases due to the inflammatory stimulus while trying to adapt its regulatory activity at $2-3 \mathrm{hr}$ postendotoxin administration. However, at $\mathrm{t}>3 \mathrm{hr}$ the activity of NF-kB cannot be regulated successfully and it settles to a sustained elevated state that drives downstream the overexcitation of both pro- and anti-inflammatory mediators; leading to an unconstrained inflammatory response. Interestingly, in (Klinke, Ustyugova et al. 2008) Klinke et al. aim at exploring experimentally the possibility of modulating the temporal control of NF-kB activation. Macrophages are exposed to a persistent inflammatory stimulus (LPS) and the available experimental data show the presence of a "damped" oscillatory behavior in NF$\mathrm{kB}$ activity. We therefore assess the appropriateness of the structure of the proposed model by simulating a malfunction in the clearance rate of pathogen-derived endotoxin, as follows. 

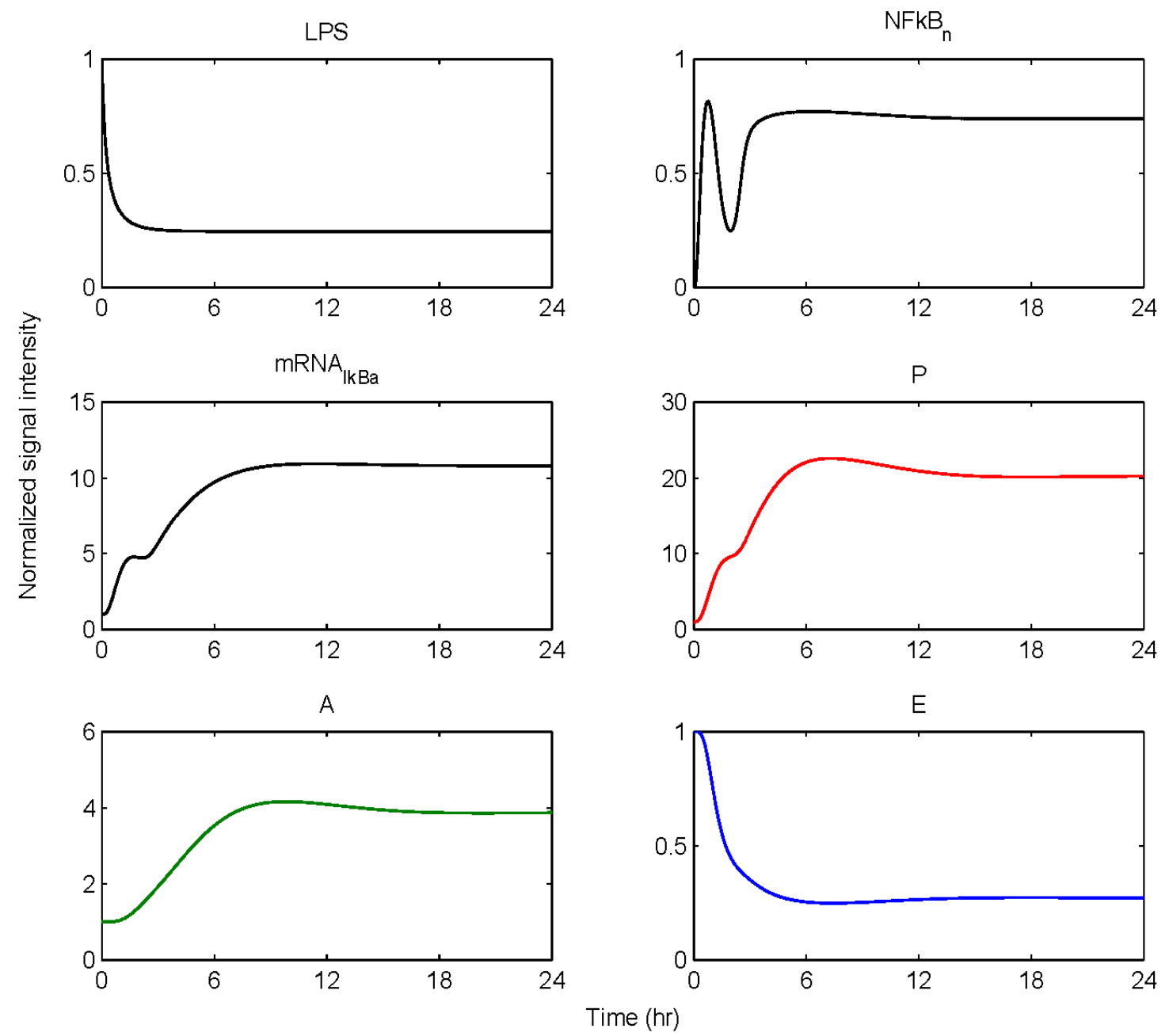

Figure 5.14: Temporal responses of model elements in a persistent infectious inflammatory response. Reducing the degradation rate of LPS to half of its initial value we simulate the case of an unsuccessful clearance of LPS that accounts for the sustained (aberrant) activity of NF-kB leading to a chronic inflammatory response.

We further evaluate the proposed in silico model by exploring the possibility of a mechanistic maladaption in the dynamics of the regulatory NF-kB signaling module. As illustrated in Figure 5.15, performing an in silico $\mathrm{IkBa}^{-/-}$knock-out experiment we simulate a sustained inflammatory response that fails to resolve. 

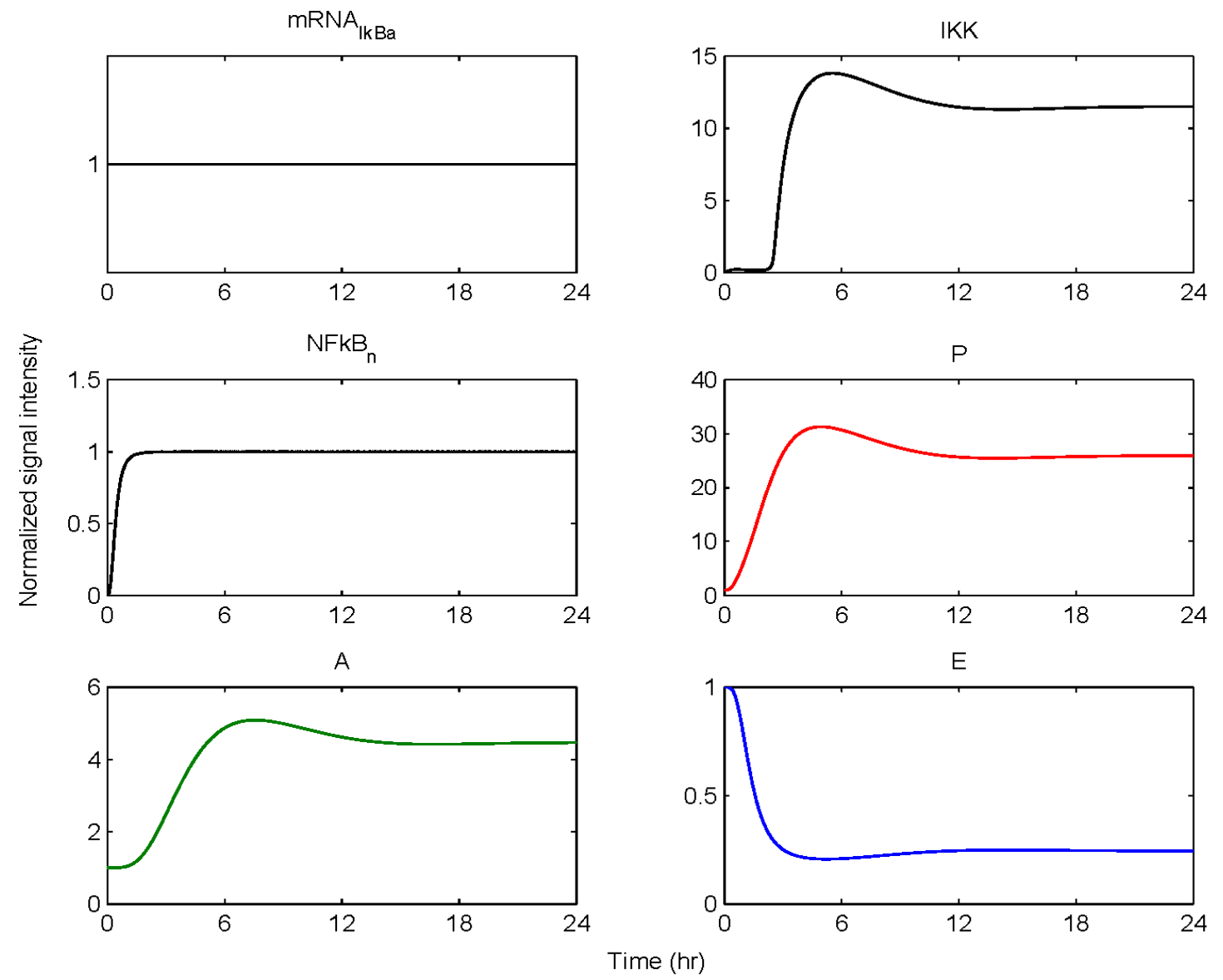

Figure 5.15: Simulation of a knock-out in silico experiment $\left(\mathrm{IkBa}^{-/-}, \mathrm{k}_{\mathrm{IkBa}, 1}=0\right)$. Manipulating the model so that there is no de novo transcriptional synthesis of NF-kB inhibitor (IkBa) which is responsible for the absence of NF-kB auto-regulatory feedback loop. Such a scenario accounts for maladapted activity of $\mathrm{NFkB}_{\mathrm{n}}$ that triggers an uncompensated inflammatory response.

The protein inhibitor of NF-kB (IkBa) aims at retrieving nuclear concentration of NF$\mathrm{kB}$ with the formation of an inactive complex in the cytoplasm regulating the expression of various inflammatory genes. The transcription factor NF-kB up-regulates the gene transcript of $\mathrm{IkBa}(\mathrm{mRNA}, \mathrm{IkBa})$ so that the translated protein IkBa serves as the major 
component for regulating its transcriptional activity. Thus, in Figure 5.15 we simulate the case of no transcriptional activity of NF-kB in the promoter region of IkBa. In the absence of NF-kB inhibitor $\left(\mathrm{IkBa}^{-/}\right)$there is an aberrant regulatory activity of NF-kB that leads to its persistent nuclear activity driving an inflammatory response that fails to restore homeostasis. Such an in silico result has been experimentally tested annotating the impact of such a knock out in inducing a chronic inflammatory response (Hoffmann, Levchenko et al. 2002b).

Another mode of perturbation of the underlying dynamics of the probed system is related to the presence of a "prior" insult that coupled with the LPS stimulus account for an overwhelming production of pro-inflammatory mediators, Figure 5.16. Such a sustained pro-inflammatory signaling deregulates the NF-kB signaling module leading to a persistent NF-kB activity. Such persistence implies that the nuclear concentration of NF-kB cannot be further constrained by its primary inhibitor, IkBa and eventually settle to a steady state far away from their equilibrium (homeostasis). We simulate such a scenario by manipulating the zero order production rate of the pro-inflammatory response $\left(\mathrm{K}_{\mathrm{in}, \mathrm{P}}\right)$ and particularly increasing it twice its initial value. A pre-existence of proinflammatory cytokines due to the presence of a prior "insult" may deregulate the intracellular dynamics responsible for an amplification of the inflammatory response. In our model such a scenario can be simulated due to the positive feedback interaction between the intracellular critical node (IKK activity) and the pro-inflammatory response that disturbs the bistable behavior of the system. Therefore we attempt to manipulate (increase) the zero order production rate of the essential pro-inflammatory signaling. Clinically, such an increased rate in the production of pro-inflammatory mediators might 
be the outcome of a surgical trauma followed by bacterial infection, a so called two hit scenario (Romascin, Foster et al. 1998).
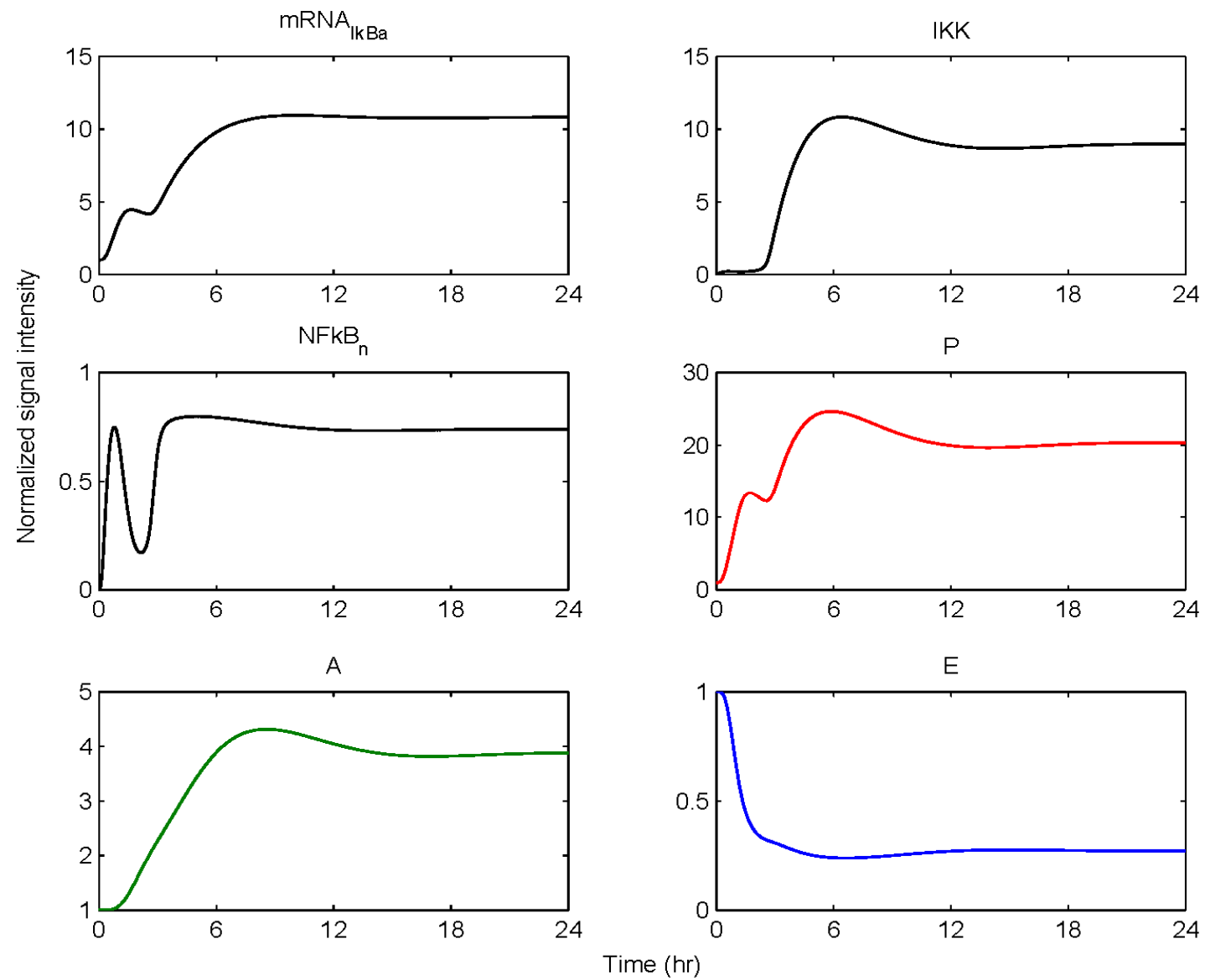

Figure 5.16: Pre-existence of pro-inflammatory mediators may enhance abnormally the intracellular signaling through IKK. Such a response leads to an unconstrained activity of NFkBn that drives downstream a persistent pro-inflammatory response which cannot be counter-regulated by the antiinflammatory arm of the host defense system. Such a mode of dysregulation is simulated by manipulating the zero production rate of pro-inflammation $\left(\mathrm{K}_{\mathrm{in}, \mathrm{P}}\right)$ so that $\mathrm{K}_{\mathrm{in}, \mathrm{P}}$ (unconstrained response) $\sim 2 * \mathrm{~K}_{\mathrm{in}, \mathrm{P}}$ (selflimited response). 


\subsubsection{Modulating the progression of an unresolved response}

The in silico model of inflammation enables us to predict an inflammatory response that does not properly abate making it a critical enabler for the evaluation of corticosteroidbased intervention strategies. Due to the physiological role of corticosteroids in the immune system (Briegel, Jochum et al. 2001) researchers have put significant effort in understanding the cytokine dynamics under hypercortisolemia (Barber, Coyle et al. 1993; Barnes and Karin 1997; Bornstein and Briegel 2003; Hawes, Rock et al. 1992; Keh, Boehnke et al. 2003; Richardson, Rhyne et al. 1989). These studies have focused on elucidating the in vivo responses to endotoxin (LPS) when there is an exposure of subjects to hypercortisolemia for various durations of time. Thus, in (Barber, Coyle et al. 1993 ) normal human subjects were exposed to glucocorticoid infusion concurrent with and before the endotoxin challenge. The hydrocortisone infusion lasted for a $6 \mathrm{hr}$ period with subsequent intervening periods of 6 (CORT-6-LPS), 12 (CORT-12-LPS) and 144hr (CORT-144-LPS) before endotoxin administration or simultaneously with LPS challenge (CORT-LPS). Experimental measurements of cytokines and hemodynamic parameters suggest the integral role of hypercortisolemia in CORT-LPS and CORT-6-LPS groups in modulating the cytokine network characterized by decreased plasma concentrations of various cytokines, i.e. TNF, IL-6 when compared to the group that received only LPS. However, in CORT-12-LPS and CORT-144-LPS the plasma concentrations of the aforementioned inflammatory mediators were significantly increased compared to CORT-LPS and CORT-6-LPS groups. Therefore, such evidence suggest the critical impact of the duration of the corticosteroid intervention before inducing inflammation in perturbing the dynamics of both hormonal and cytokine level. 
Herein, we explore the capability of corticosteroids to modulate the inflammatory response under various treatment schedules. An intravenous injection of the drug, via the activation of intermediate signaling steps, eventually leads to the up-regulation of the active complex, FR(N). Based on the mode of corticosteroids action defined, we explore the potential of the active signal, $\mathrm{FR}(\mathrm{N})_{\text {norm, }}$, in modulating the progression of an unresolved inflammation, Figure 5.17. We observe that such a signal mediates the corticosteroid effect on the transcriptional response level primes the dynamic state of NF$\mathrm{kB}$ inhibitor so that it suffices to promote resolution of the inflammatory response. Despite the high initial LPS concentration which perturbs the dynamics of inflammation (dashed lines), the corticosteroid intervention in the form of an intravenous (i.v.) injection initiated at $\mathrm{t}=0 \mathrm{hr}$ "reprograms" the dynamic state of the system in favor of a balanced regulation (solid lines). While comparing the dashed and solid lines in Figure 5.17 we observe that the intervention strategy plays a critical role in the dynamics of IkBa during the first 4 hrs post-LPS where suffices to control the intrinsic inflammatory dynamics favoring homeostasis within $24 \mathrm{hrs}$. On the other hand, prior to any intervention the system seems to have lost any potential for attenuation and its inability to adapt to high LPS concentration is mathematically translated into unconstrained responses (dashed lines). Therefore, the intervention envelope based on corticosteroids serves as a critical enabler to explore the capability of different intervention strategies in modulating the progression of systemic inflammation. 

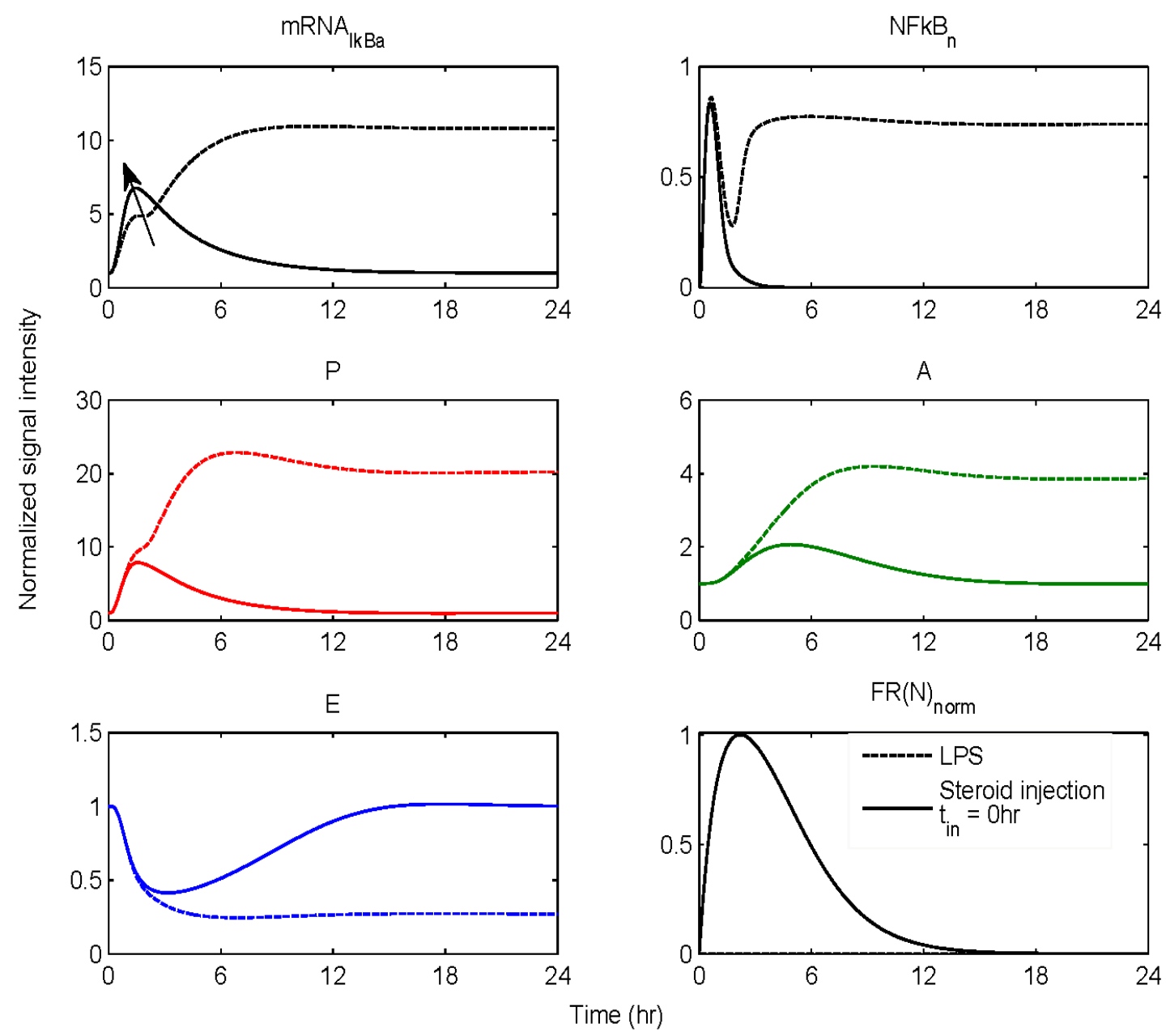

Figure 5.17: Exploring the mode of corticosteroid action in enhancing the transcriptional synthesis of IkBa which is illustrated by the solid arrow. An i.v. injection of the corticosteroid drug administered concomitantly with endotoxin $\left(\mathrm{t}_{\text {in }}=0 \mathrm{hr}\right)$ suffices to reverse (prevent) the lethal effect of a high dose of endotoxin. Solid lines (-) correspond to the inflammatory resolution due to the corticosteroid injection at $\mathrm{t}$ $=0 \mathrm{hr}$ while dashed lines simulate the progression of inflammation in response to a high concentration of LPS (i.e. $\operatorname{LPS}(\mathrm{t}=0 \mathrm{hr})=4)$. 
As shown in Figure 5.17 a single i.v. injection of corticosteroids at $\mathrm{t}=0 \mathrm{hr}$ suffices to reverse the dynamics in response to the high concentration of LPS. Similar results were obtained if we preserved the timing of intervention but modified the route of drug administration switching to a continuous infusion, Figure 5.18.
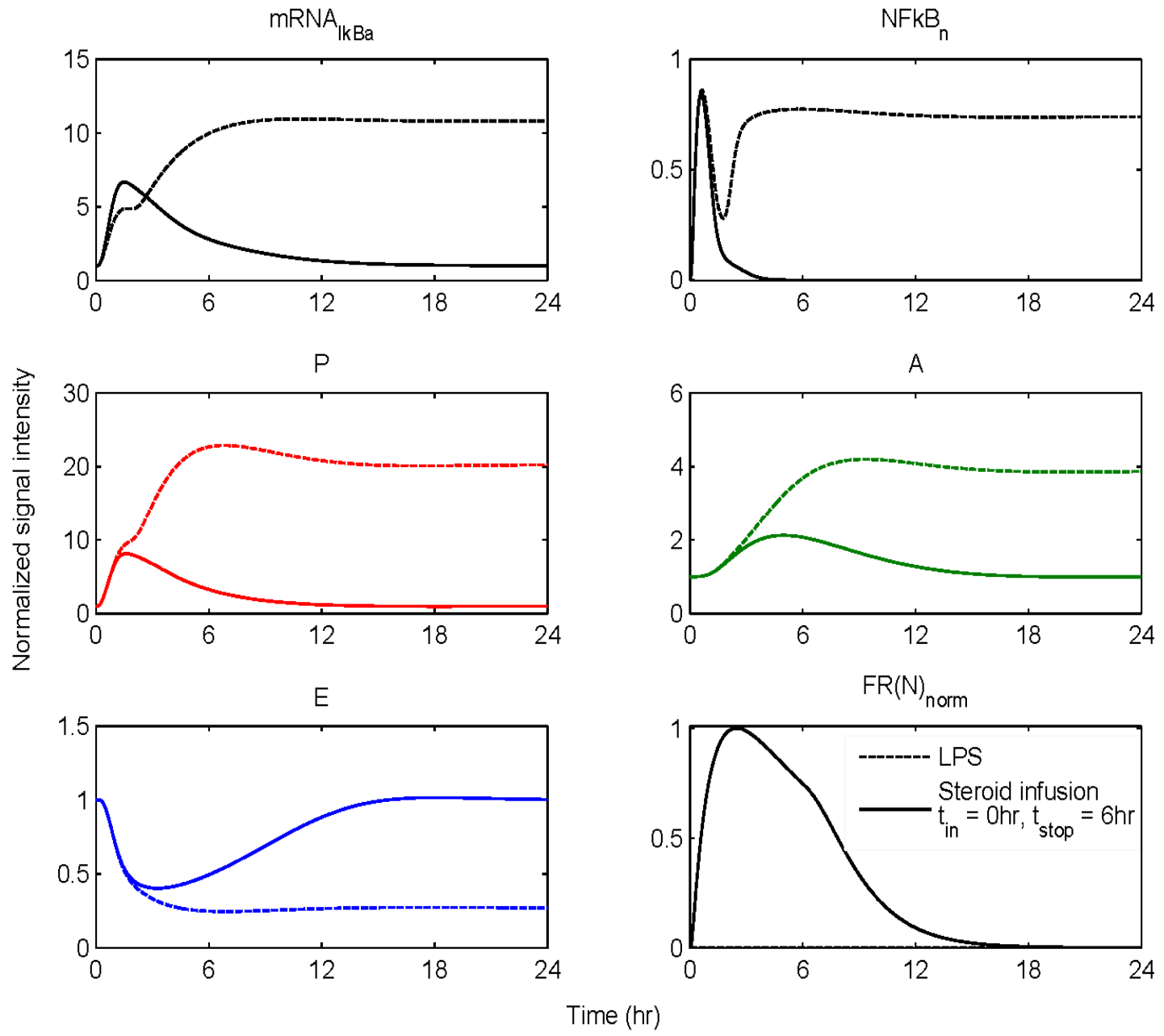

Figure 5.18: Exploring the effect of steroid infusion initiated simultaneously with LPS injection $\left(\mathrm{t}_{\mathrm{in}}=0 \mathrm{hr}\right)$ and continued for $6 \mathrm{hr}\left(\mathrm{t}_{\text {stop }}=6 \mathrm{hr}\right)$ after LPS exposure in modulating the progression of unremitting inflammation evoked by high LPS dose. Solid lines (-) correspond to the inflammatory resolution due to the corticosteroid infusion while dashed lines simulate the progression of aberrant inflammation in response to high LPS challenge. 
As seen in Figure 5.18 such treatment strategy suffices to reverse the deleterious outcome of a persistent non-infectious inflammatory response (due to high initial LPS concentration). Moreover, pre-exposing the system before endotoxin challenge for $6 \mathrm{hr}$ to hypercortisolemia we observe a proper modulation on the progression of the inflammatory response as well, Figure 5.19.
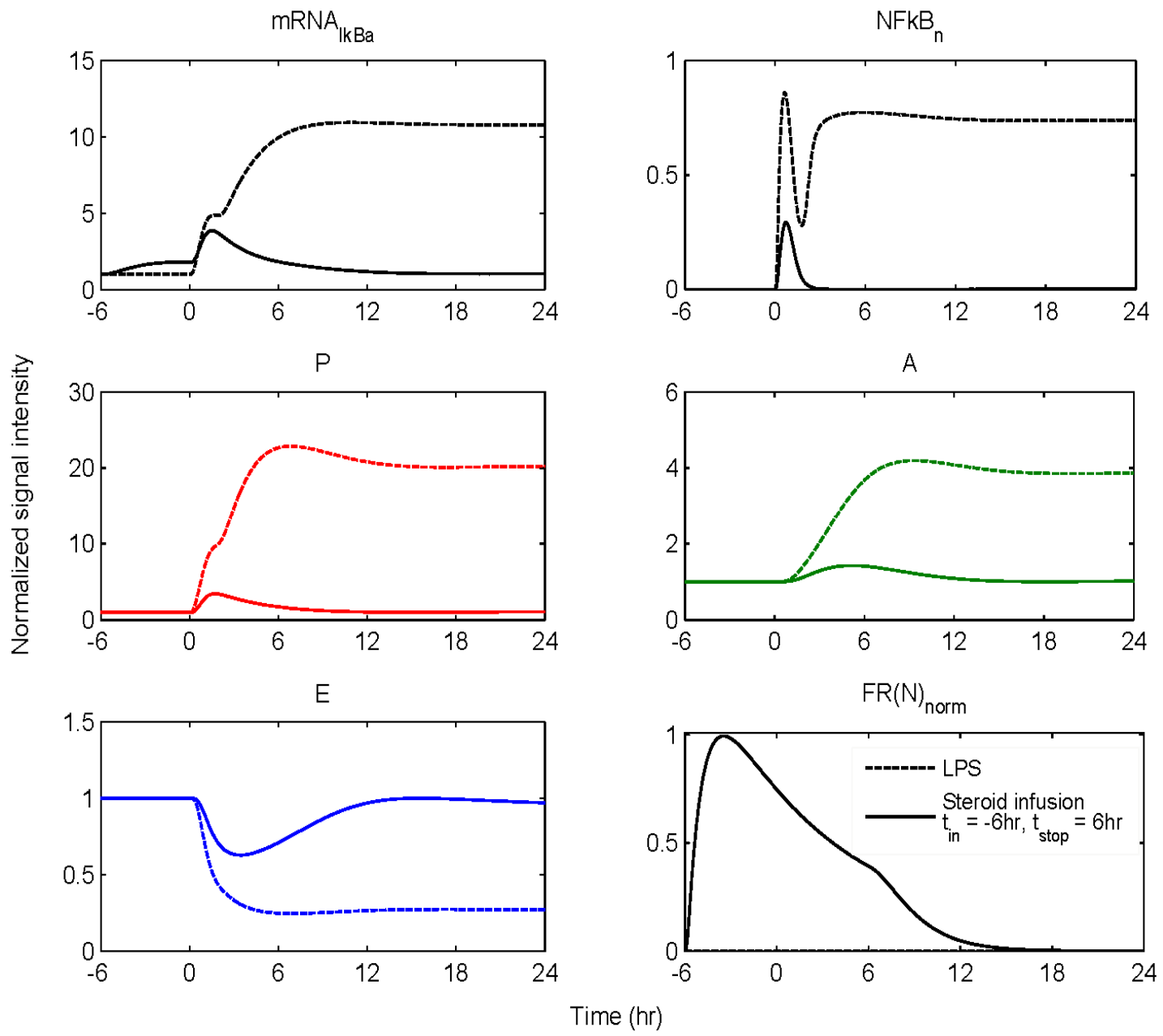

Figure 5.19: Hypercortisolemia for $6 \mathrm{hr}$ prior to LPS challenge $\left(\mathrm{t}_{\mathrm{in}}=-6 \mathrm{hr}\right)$. The system is pre-exposed for $6 \mathrm{hr}$ to a continuous infusion of corticosteroids while it is continued for another $6 \mathrm{hr}$ after the endotoxin challenge $\left(t_{\text {stop }}=6 \mathrm{hr}\right)$. Such an intervention "reprograms" the dynamics of the system modulating the effect of a high LPS concentration. 
In addition to Figure 5.19, similar responses are observed for the system if it is preexposed to hypercortisolemia for $6 \mathrm{hr}$ but the steroid intervention is initiated at $\mathrm{t}=-12 \mathrm{hr}$. Such results support an early intervention strategy that targets the regulatory arms of systemic inflammation and successfully capture the dynamic behavior of the system in CORT-LPS and CORT-6-LPS groups of the aforementioned experimental study. Complementary to this, in (Sato, Koeda et al. 2002) there is emphasis on the potential of a preoperative administration of corticosteroids in alleviating surgical stress. The underlying hypothesis of such a preoperative exposure stems from the fact that a modification of the inflammatory dynamics at an early stage (transcriptional level) would seem to be beneficial in balancing the immune response given that these antiinflammatory drugs (corticosteroids) inhibit pro-inflammatory transcription factors (NF$\mathrm{kB})$.

However, if the system is pre-exposed to hypercortisolemia for the same duration as previously mentioned (6hr) but the time interval between the termination of infusion and LPS administration is much greater (i.e. $>12 \mathrm{~h}$ ), we observe a blunted effect of the corticosteroid treatment on the progression of inflammation (Note that the drug has been eliminated from the host), Figure 5.20. Such an intervention strategy fails to reverse the effects of a high concentration of LPS. Similar results are observed if the intervention strategy elapses at times greater than $12 \mathrm{hr}$ from LPS administration (data not shown here). The primary reason for such a failure stems from the fact that at $\mathrm{t}>12 \mathrm{hr}$ the transcriptional profile of anti-inflammatory molecules i.e. (A) and (IkBa) are resolved. Therefore any pre-exposure to corticosteroid infusion that is terminated at $>9 \mathrm{hr}$ would 
not "reprogram" the inflammatory dynamics towards a reversal in the progression rate of an inflammatory response.

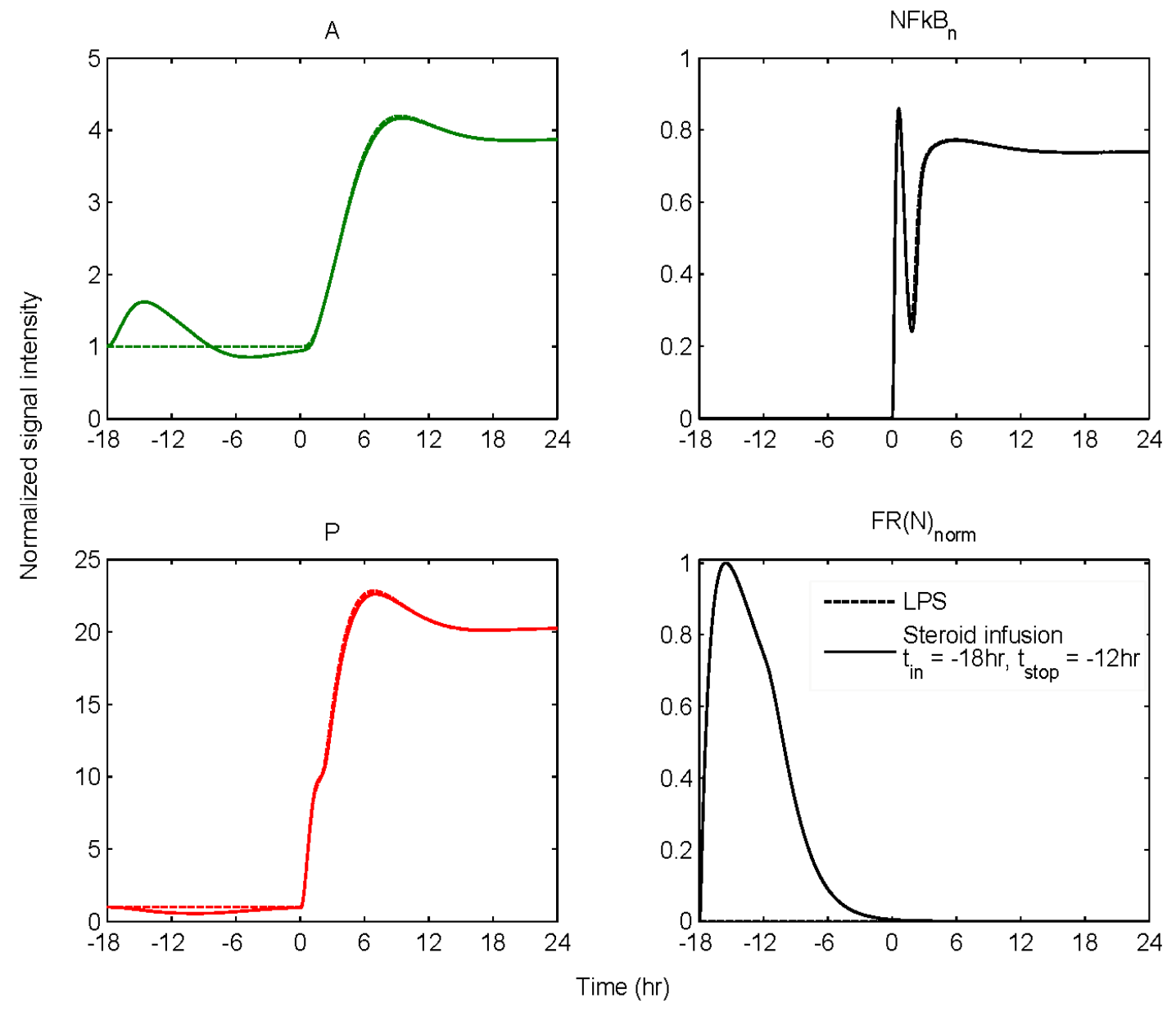

Figure 5.20: Pre-exposure the system into hypercortisolemia which is assumed to modulate the dynamics of IL-10 signaling (A). Such intervention strategy is initiated $18 \mathrm{hr}$ before the endotoxin challenge and continued for $6 \mathrm{hr}$ while it does not have a profound effect in the dynamic state of the system where the progression of an unresolved inflammation ( - lines) continues after the termination of steroid infusion (represented by overlap between solid and dashed lines). 
Similar results in the context of no reversibility in the host dynamics are obtained if the system is exposed to a continuous infusion of hypercortisolemia initiated at $\mathrm{t}>0 \mathrm{hr}$ after the administration of endotoxin (i.e. $\mathrm{t}=1 \mathrm{hr}$ ), dashed and dotted lines, Figure 5.21.
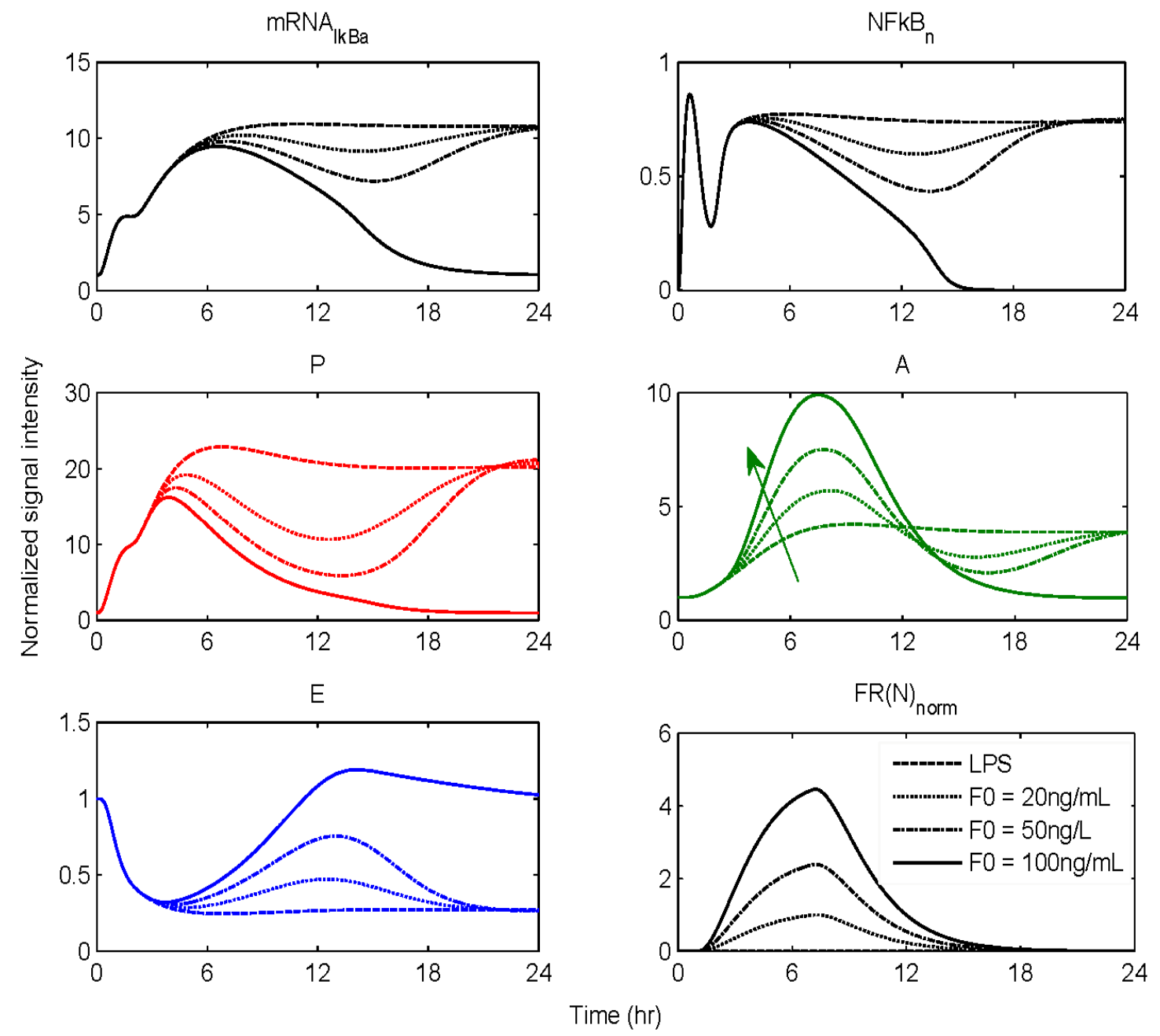

Figure 5.21: Explore the effect of corticosteroids at multiple drug doses initiated at $\mathrm{t}=1 \mathrm{hr}$ and continued for $6 \mathrm{hr}$ after the endotoxin challenge priming the production rate of IL10 signaling (A component) illustrated by green arrow. Solid lines characterize a resolution in the progression of systemic inflammation whereas dashed and dotted lines correspond to lower drug doses that cannot sufficiently reverse the progression rate of an aberrant inflammation. All the active signals, FR(N)norm, have been normalized with respect to the lowest drug dose, $\mathrm{F} 0=20 \mathrm{ng} / \mathrm{mL}$ (represented by dotted lines). 
Exploring the response of the system at later stages of the progression of the inflammatory response, Figure 5.21, the active steroid signal, FR(N) norm, has to increase in magnitude in order for the inflammatory response to be tightly regulated. Thus, the progression of the inflammatory response is differently perturbed on a dose-dependent manner (dotted and dashed lines versus solid). Preserving the route of drug administration the active signal $\mathrm{FR}(\mathrm{N})$ must increase in magnitude in order for the system to respond to a multitude of external signals (LPS, Drug).Therefore, dose-dependent profiles are simulated in the aforementioned figure where there exists a dosage regime that modulates the dynamics of the system towards resolution.

These in silico results lie in agreement with studies (van der Poll, Barber et al. 1996) that suggest a dose-dependent decrease in LPS-induced TNF in peripheral human blood leukocytes that are exposed to hydrocortisone infusion. In particular, van der Poll and Lowry (van der Poll, Barber et al. 1996) demonstrate increased plasma concentrations of IL10 at higher corticosteroid doses. In our model, if we assume that corticosteroids instead of up-regulating the inhibitor of NF-kB they prime the production rate of IL10 signaling (A), the gradual increase in the anti-inflammatory (A) signaling as the drug dose increases modulates the response of the system towards a more balanced inflammatory response. In addition, the computational experiments presented in Figure 5.17 - Figure 5.19 can be reproduced if we consider that the corticosteroid intervention envelope perturbs the state of the anti-inflammatory (A) signaling which lie in agreement with the pleiotropic mode of corticosteroids anti-inflammatory activity. Despite the controversies regarding the administration of either high-dose steroids for the short-term in septic patients (Lefering and Neugebauer 1995) or the prolonged use of low dose 
steroids in clinical settings (Klaitman and Almog 2003) the present study provides qualitative insight on how the system responds to various intervention strategies opening challenging windows towards the design of effective drug treatment schedules (Zurakowski and Teel 2006).

In summary we have developed a semi-mechanistic host response model that describes the dynamic evolution of an in vivo human response to endotoxin. Interacting components involve elementary signaling pathways that propagate extracellular signals to the transcriptional response level and pharmacokinetic models of corticosteroids, as putative controllers of the inflammatory response. Model parameters are appropriately evaluated so that to reproduce a self-limited inflammatory response that resolves within $24 \mathrm{hr}$ post-endotoxin administration. The potential of the model is demonstrated via computational tests performed to reproduce biologically relevant scenarios associated with an increase in host's susceptibility to endotoxin stimulus as well as in the regulatory interactions of signaling cascades. Exploring the possible effects of systemic perturbations enables us to trace the dynamics of a systemic inflammatory response syndrome. In silico experiments that activate the corticosteroid intervention envelope in order to modulate the progression of inflammation, encourage the proper design of intervention strategies that target early arms of the host response modulating the activity of crucial pro-inflammatory transcription factors. Such a modeling framework can potentially offer significant insight as to how a host undergoing an inflammatory response responds to a multitude of external signals through interacting signaling modules and possible strategies for restoring homeostasis. 


\subsection{Appendix to the NF-kB dependent physicochemical model}

\subsubsection{Table of relevant model components}

\begin{tabular}{|l|l|l|l|}
\hline Symbol & Description & Symbol & Description \\
\hline IKK & Kinase activity & $\mathrm{F}$ & Drug (Cortisol) \\
\hline mRNA $_{\text {IkBa }}$ & Gene transcript of NF-kB inhibitor & $\mathrm{R}_{\mathrm{m}}$ & Gene transcript of steroid \\
& $(\mathrm{IkBa})$ & receptor \\
\hline IkBa & Protein inhibitor IkBa & $\mathrm{R}_{\mathrm{F}}$ & Free cytosolic steroid \\
\hline $\mathrm{NFkB}$ & Nuclear concentration of NF-kB & FR & Cytosolic steroid-receptor \\
\hline FR(N) & Nuclear steroid-receptor complex & & complex \\
\hline
\end{tabular}

\subsubsection{Estimated parameter values}

Table 5.2: Estimated values of parameters based on self-limited human data ${ }^{4}$

\begin{tabular}{|c|c|c|c|}
\hline $\mathrm{k}_{3}=5.000$ & $\mathrm{~K}_{\text {out }, \mathrm{IkBa}}=0.463$ & $\mathrm{~K}_{\mathrm{in}, \mathrm{P}}=0.033$ & $\mathrm{k}_{\mathrm{A}, \mathrm{E}}=5.300$ \\
\hline $\mathrm{k}_{4}=2.240$ & $\mathrm{k}_{\mathrm{I}, \mathrm{I}}=1.400$ & $\mathrm{k}_{\mathrm{P}, \mathrm{NFkBn}}=29.741$ & $\mathrm{~K}_{\text {out }, \mathrm{A}}=0.592$ \\
\hline $\mathrm{k}_{\mathrm{NFkB}, 1}=16.294$ & $\mathrm{k}_{\mathrm{I}, 2}=0.870$ & $\mathrm{k}_{\mathrm{P}, \mathrm{E}}=9.050$ & $\mathrm{~K}_{\mathrm{in, \textrm {E }}}=0.080$ \\
\hline $\mathrm{k}_{\mathrm{NFkB}, 2}=1.186$ & $\mathrm{~K}_{\mathrm{in}, \mathrm{mRNAR}, \mathrm{R}}=0.091$ & $\mathrm{~K}_{\mathrm{out}, \mathrm{P}}=0.333$ & $\mathrm{k}_{\mathrm{E}, \mathrm{P}}=2.217$ \\
\hline $\mathrm{K}_{\mathrm{in}, \mathrm{IkBa}}=0.463$ & $\mathrm{k}_{\mathrm{mRNAR}, \mathrm{P}}=1.740$ & $\mathrm{~K}_{\mathrm{in}, \mathrm{A}}=0.093$ & $\mathrm{~K}_{\text {out }, \mathrm{E}}=0.257$ \\
\hline $\mathrm{k}_{\mathrm{IkBa}, 1}=13.273$ & $\mathrm{~K}_{\text {out,mRNA,R }}=0.2505$ & $\mathrm{k}_{\mathrm{A}, \mathrm{P}}=0.010$ & \\
\hline
\end{tabular}

\footnotetext{
${ }^{4}$ Parameter values $\mathrm{k}_{\mathrm{lps}, 1}, \mathrm{k}_{\mathrm{lps}, 2}, \mathrm{k}_{\mathrm{syn}}, \mathrm{k}_{1}$ and $\mathrm{k}_{2}$ are maintained to agree with those presented in Table 5.1 (see Appendix 5.2)
} 
5.4.3 Fixed parameter values involved in cortisol dynamics ${ }^{5}$

\begin{tabular}{|c|c|c|}
\hline $\mathrm{C}_{1}=39130$ & $\mathrm{IC}_{50 \_\mathrm{Rm}}=26.200$ & $\mathrm{k}_{\mathrm{on}}=0.003$ \\
\hline $\mathrm{C}_{2}=12670$ & $\mathrm{k}_{\mathrm{deg}}=0.112$ & $\mathrm{k}_{\mathrm{dgr} \_}=0.057$ \\
\hline $\mathrm{k}_{1}=7.540$ & $\mathrm{k}_{\mathrm{syn} \_\mathrm{R}}=1.199$ & $\mathrm{k}_{\mathrm{T}}=0.630$ \\
\hline $\mathrm{k}_{2}=1.200$ & $\mathrm{r}_{\mathrm{f}}=0.490$ & \\
\hline $\mathrm{k}_{\mathrm{syn} \_\mathrm{Rm}}=2.900$ & $\mathrm{k}_{\mathrm{re}}=0.570$ & \\
\hline
\end{tabular}

\subsubsection{Initial conditions of relevant model components}

\begin{tabular}{|c|c|c|c|c|}
\hline $\operatorname{IKK}(0)=0$ & $\operatorname{mRNA}_{\mathrm{IkBa}}(0)=1$ & $\mathrm{~F}(0)=\mathrm{C}_{1}+\mathrm{C}_{2}{ }^{*}$ & $\mathrm{R}_{\mathrm{m}}(0)=25.8$ & $\mathrm{FR}(0)=0$ \\
\hline $\mathrm{NFkB}_{\mathrm{n}}(0)=0$ & $\mathrm{IkBa}(0)=0$ & $\mathrm{~F}(0)=0$ (infusion) & $\mathrm{R}_{\mathrm{F}}(0)=540.7$ & $\mathrm{FR}(\mathrm{N})(0)=0$ \\
\hline
\end{tabular}

${ }^{*} F(0)=51800 \mathrm{ng} / \mathrm{mL}$ for injection as reported in the original analysis (Jin, Almon et al. 2003)

\subsubsection{Steady-state baseline equations}

\begin{tabular}{|c|c|}
\hline Eq. (4.32) & 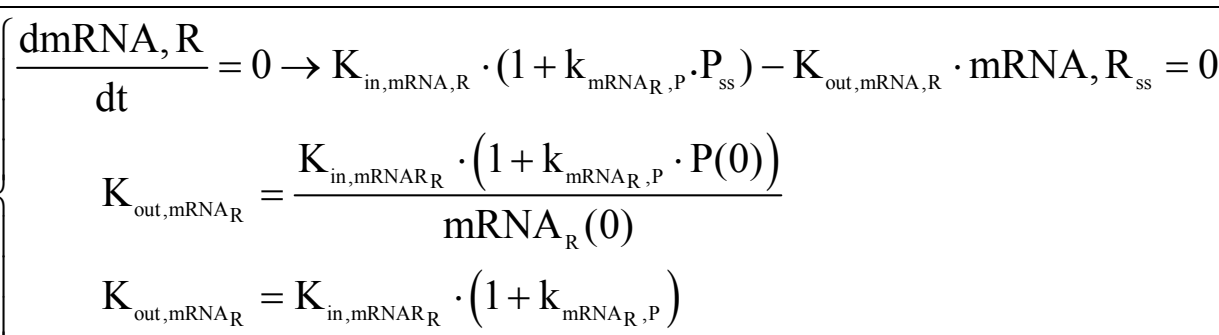 \\
\hline Eq. (4.33) & $\left\{\begin{aligned} \frac{\mathrm{dmRNA}_{\mathrm{IkBa}}}{\mathrm{dt}} & =0 \rightarrow \mathrm{K}_{\mathrm{in}, \mathrm{IkBa}} \cdot\left(1+\mathrm{k}_{\mathrm{IkBa}, 1} \cdot \mathrm{NFkB}_{\mathrm{n}, \mathrm{ss}}\right)-\mathrm{K}_{\text {out }, \mathrm{kkBa}} \cdot \mathrm{mRNA}_{\mathrm{IkBa}, \mathrm{ss}}=0 \\
\mathrm{~K}_{\text {out }, \mathrm{IkBa}} & =\frac{\mathrm{K}_{\mathrm{in}, \mathrm{IkBa}} \cdot\left(1+\mathrm{k}_{\mathrm{IkBa}, 1} \cdot \mathrm{NFkB}_{\mathrm{n}}(0)\right)}{\mathrm{mRNA}_{\mathrm{IkBa}}(0)} \\
\mathrm{K}_{\text {out }, \mathrm{IkBa}} & =\mathrm{K}_{\mathrm{in}, \mathrm{lkBa}}\end{aligned}\right.$ \\
\hline
\end{tabular}

\footnotetext{
${ }^{5}$ Parameter $\mathrm{R}_{\text {in }}$ for infusion kinetics is defined as (drug_dose $* \mathrm{k}_{1}$ )/duration where $\mathrm{k}_{1}$ and duration represent elimination rate and duration of infusion respectively.
} 


\subsection{An agent-based model of acute human inflammation}

Biological systems, unlike physical or chemical systems are characterized by the emergence of inhomogeneous distribution of their components (Bauer, Beauchemin et al. 2009). The recognition that equation based models are predicated on the assumption of a homogeneously distributed system has made it less applicable in situations where spatial effects are important (An, Faeder et al. 2008). On the other hand, agent based models $(\mathrm{ABM})$ has an intrinsically spatial component based on its reliance upon local interactions and environmental heterogeneity. Although both modeling approaches (EBM and ABM) have both advantages and disadvantages (Vodovotz, Clermont et al. 2004), agent based modeling has emerged as an alternative for addressing features of complex biological systems (Ermentrout and Edelstein-Keshet 1993).

To examine the effects of the assumption of spatial heterogeneity, there is a growing body of research probing the effects of spatial distribution in the innate immune system (Funk, Jansen et al. 2005; Louzoun, Solomon et al. 2001). Thus, a central premise of $\mathrm{ABM}$ is that they map intuitively to biological phenomena such as cells within tissues and organs capturing the stochastic nature and dynamic transitional states in biological systems (An 2006; Lowry and Calvano 2008; Neugebauer, Willy et al. 2001; Seely and Christou 2000). In addition to this, the ABM approach provides a very intuitive means of translation of basic science data on the innate immune response through a series of simple rules that dictate their behaviors. Accordingly, a number of excellent prior studies have placed significant emphasis on simulating the dynamics of inflammation predicated upon the principles of agent based models (Baldazzi, Castiglione et al. 2006; Clermont, Bartels

et al. 2004; Kataoka, Ito et al. 2004; Mi, Riviere et al. 2007; Vodovotz, Chow et al. 
2006). Specifically, in the studies conducted by An and collaborators (An 2001; An, Hunt et al. 2007; An 2008; An, Faeder et al. 2008), the applications of ABM in inflammation models have been effectively demonstrated.

The key elements in ABMs are the agents, which are entities that represent a certain aspect of the system, for instance a family of cells and/or molecules that are able to adapt and interact with the environment and with each other based on a specific set of rules (Vodovotz, Csete et al. 2008). While agents within a class will have the same rules for behavior, the behavior of individual agents varies because of differences in local conditions. The individual interactions then aggregate to engender the overall behavior observed in an experimental setting. The advantage of ABMs lies in the fact that the interactions of agents are derived from fundamental occurrences in biological processes, like the binding of molecules, and as such, they are more intuitive to implement and easier to understand. Additionally, the instructions that describe the interactions are taken from published literature and translated into programming language. Furthermore, the model is naturally stochastic in that the interactions can be designed to be based upon probabilities and some of the agent dynamics can be highly random.

The work to be discussed in this section seeks to address the possibility of an agent based modeling approach that defines the propagation of a perturbation across the system taking into account spatial orientation at the molecular level as well as cellular interactions and heterogeneity. Driven by the premise that peripheral blood leukocytes (PBLs) are major effectors in response to endotoxin and that PBLs represent a composite mixture of several cellular subpopulations we opted to simulate the stochastic interactions particularly in the macrophages and $\mathrm{T}$ helper cells. During the onset of the inflammatory 
response, the secretion of pro-inflammatory cytokines from macrophages stimulates the activation of precursor T helper cells (Th0) and induce them to exhibit the type $1 \mathrm{~T}$ helper cell (Th1) phenotype thatin turn facilitates the secretion of various pro-inflammatory cytokines (McKnight, Zimmer et al. 1994). The other fate of Th0 is to become type $2 \mathrm{~T}$ helper cells (Th2) and produce anti-inflammatory cytokines that are essential for restoring homeostasis (Kidd 2003). Physiologically, the recruitment of macrophages and the differentiation of Th cells occur in separate locations. Yet they retain strong interconnectivity facilitated by the inflammatory cytokines. Due to limitation of the framework, the proposed model did not separate the aforementioned cell types into different topological compartments. We assumed however that the movement of the cytokine agents from the macrophages to the Th cells would signify the transportation of the cytokines between different biological tissues. Previous agent based studies have placed emphasis on simulating either intercellular interactions between a multitude of such cell types (Folcik, An et al. 2007) or the spatial orientation of molecules involved in the NF-kB signaling pathway (Pogson, Holcombe et al. 2008) while considerable attention has been also given to modeling the transcriptional regulatory network of $\mathrm{TH}$ differentiation (Santoni, Pedicini et al. 2008).

In this section we have taken an integrative approach to elucidate molecular interactions involved in the NF-kB signaling pathway, coupled with the spatial orientation of various inflammation specific molecules and cell populations such as macrophages and T-helper cells. Our agents of choice reflected the characteristics of biological molecules. This allowed us to focus on the intracellular dynamics of the NF$\mathrm{kB}$ signaling module and further illustrate the subsequent intercellular interactions 
through the up-regulation of inflammatory mediators. The stochastic behavior of the agents was partially attributed to the random motion of the molecules. The probability that determined whether an interaction should occur relied on the spatial configuration of the participants. Cells were not considered as reactor spaces with an even distribution of molecules. To accommodate for this, some of the agent-based rules regarding the mobilization of molecules were implemented, in order to ensure that a specific interaction occurs within an allocated time frame and the network topology of the model. A key characteristic of our approach was to represent the cellular interactions as the aggregated output of an intricate process that influenced the cellular behavior and therefore the overall systemic response.

\subsubsection{Elements of the agent based host response model of human inflammation}

At the transcriptional response level, we have previously demonstrated (Chapter 4) that the transcriptional dynamics of human leukocytes exposed to bacterial endotoxin can be decomposed into to three elementary comprehensive responses. These responses defined the major (essential) transcriptional elements of the host response to endotoxin that subsequently manifest the integrated systemic response. In an attempt to establish quantifiable relationships among these essential components of human endotoxemia we have proposed the development of deterministic, semi-mechanistic based host response models that include transcriptional dynamics, signaling and physiological components for the modulation of the response (Section 5.1 and 5.3). Herein, we have sought to describe the host response to endotoxin via interacting molecules and cells based on an integrated ABM framework as shown in Figure 5.22. 


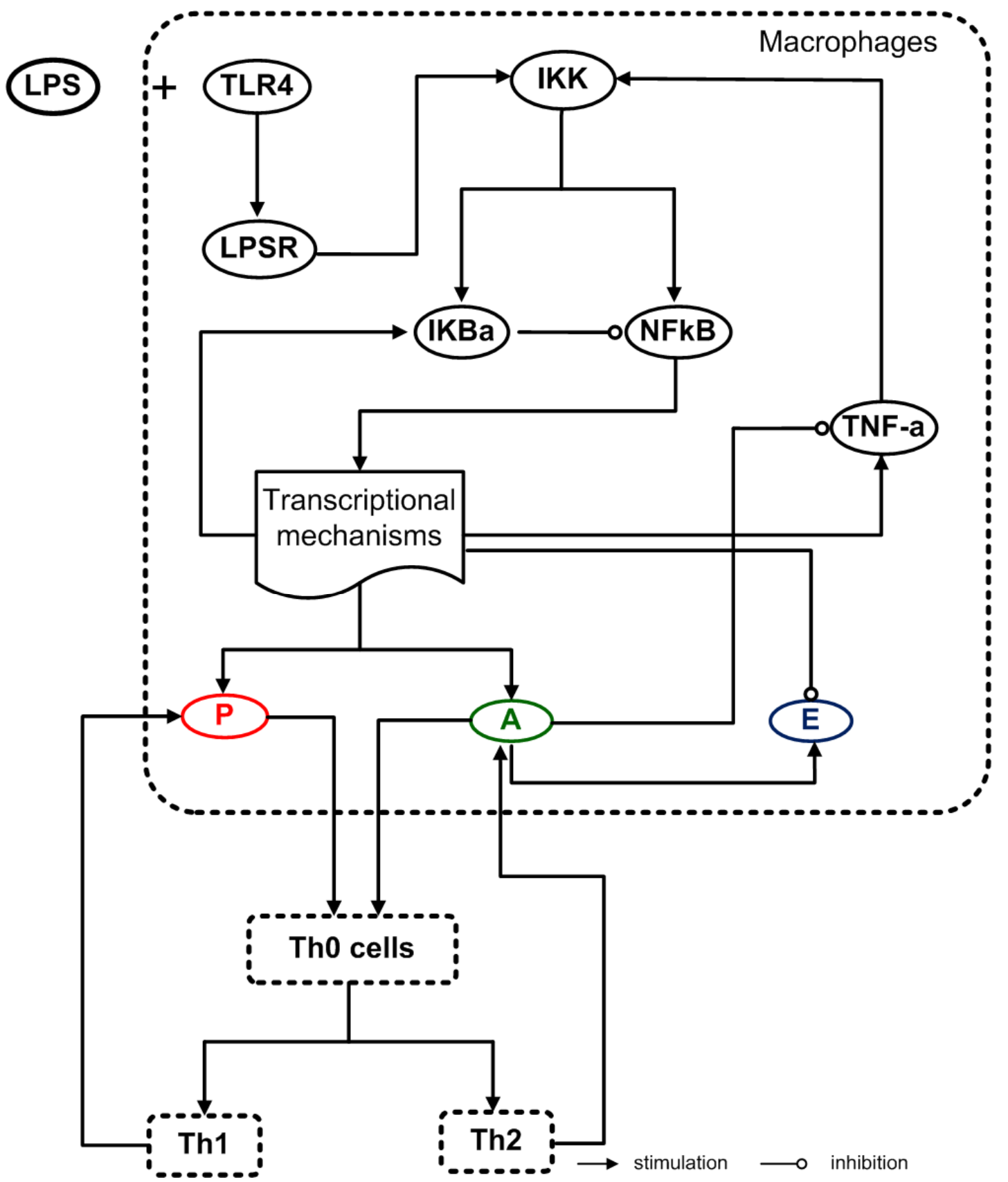

Figure 5.22: Schematic illustration of elements and interactions involved in the agent based model of endotoxin induced inflammation. 
Each macrophage possessed a cell membrane comprised of agents arranged in a circle around the center of the cell which constitutes the nucleus. Receptors for LPS, IL-4, and TNF-alpha were embedded in the membrane while the inhibitor protein IkBa, IKK, and NF-kB are located in the cytosol. Prior to any external perturbation, NF-kB is inactive in the cytoplasm forming a complex with its primary inhibitor, IkBa. Upon stimulation, NF$\mathrm{kB}$ translocates to the nucleus activating the transcriptional machinery for the upregulation of the critical transcriptional events [27-29]. During the recognition process of LPS from its signaling receptor (R), a signal transduction cascade is triggered that upregulates the transcription of TNF-a. Since pro-inflammatory cytokines might be responsible for perpetuating and amplifying the inflammatory reaction through the critical node (IKK) (Karin and Delhase 2000), such interaction is simulated via the positive interaction between TNF-a, and the kinase activity (IKK).

Consequently, the presence of pro-inflammatory mediators (P) promoted the migration of mature T helper cells (Mackay 2000) where Th0 cells become Th1, while the production of anti-inflammatory mediators (A) incited formation of Th2 cells which further potentiate the anti-inflammatory response (A) (Kidd 2003). Since the antiinflammatory arm of the host defense system restores homeostasis, the anti-inflammatory component of the model, including anti-inflammatory mediator agents (A), was assumed to exert its counter-regulatory properties by stimulating the degradation rate of the early potent pro-inflammatory mediator TNF-a, coupled with the active populations of $\mathrm{T}$ helper cells. In particular, the Th2 agents continuously produced anti-inflammatory mediators to ensure that the population of (A) agents was sufficient to attenuate TNF-a production in macrophages. Since circulating pro-inflammatory $(\mathrm{P})$ agents have the ability to turn Th0 
into Th1, instead of $\mathrm{Th} 2$, the population of Th2 cells is primarily affected by the concentration of $(\mathrm{P})$ agents. Therefore, the resolution of the inflammatory response is highly dependent on the balance between pro- and anti-inflammatory mediators that are additionally regulated by the energetic state of macrophages. To establish the link between the inflammatory response and the cellular energetic state, we assumed that upon activation of a pre-defined threshold the essential energetic response was assumed to subsequently modulate the degradation rate of TNF-a (Gupta and Gollapudi 2005). The production rate of the inflammatory mediator TNF-a increased when the energetic state was lowered during the progression of the inflammatory reaction by NF-kB. Meanwhile, the presence of anti-inflammatory mediators leads to a decrease in the proximal inflammatory mediator, TNF-a.

\subsubsection{Developing an agent based model of endotoxin induced human inflammation}

The inflammatory response is activated when endotoxin is recognized by pathogen recognition receptors (Wells, Ravasi et al. 2005). Such recognition process involves the induction of a signal transduction cascade that triggers downstream critical signaling modules for the activation of transcriptional factors that play a critical role for the transcriptional initiation of inflammatory genes. LPS molecules collide with their receptor, TLR4, on the surface of the macrophages. If the receptor is unoccupied, the LPS molecule will have a probability to bind to its signaling receptor, forming a complex. A receptor that is already bound to a LPS molecule will be unable to receive another one. The bound receptor is also considered activated, in that it will up-regulate the production of TNF-a molecules stimulating downstream intricate signaling cascades. Such a cascade involves the activation of kinase (IKK) activity, which in turn phosphorylates the 
inhibitor protein IkBa and leads to the activation of the transcription factor NF-kB. The transcriptional end result of this signaling pathway is the production of pro-inflammatory cytokines including IL-12, TNF-a, and IkBa. The IkBa molecules effectively terminate the pathway by forming an inactive complex with nuclear NF-kB in the cytosol. The production of IL-12 initiates the production of IL-4 molecules. These two cytokines populate the system and bind to their respective receptors on the macrophages or type T0 helper cells. The fate of Th-0 cells is determined by the number of either IL- 4 or IL-12 on its surface receptors (Kidd 2003).

The pro- and anti-inflammatory mediator profiles ( $\mathrm{P}$ and $\mathrm{A}$ ) and the energetic response of the macrophages (E) were used as a primary indication of a constrained or unresolved inflammatory response. During the progression of systemic inflammation, pro-inflammatory $(\mathrm{P})$ molecules specifically reflect the presence of IL-12 mediators that are circulating in the system. The primary reason for such a selection stems from the fact that the role of IL-12 has been implicated in the differentiation of Th- 0 cells. However, each essential transcriptional signature $(\mathrm{P}, \mathrm{A}, \mathrm{E})$ as it previously mentioned, serves as the aggregate signal that describes complex inflammatory reactions. Thus, (P) would qualitatively reflect the secretion of cytokines and chemokines such as TNFSF2 (TNF), IL1A, IL1B, CXCL1, CXCL2, CCL2, CXCL8 (IL-8) and CXCL10. Similarly, the antiinflammatory arm of the system (A) reflects either the number of IL-4 molecules upon endotoxin simulation or mediators such as IL1RAP, IL1R2, IL10 and TNFRSF1A. We would like to comment that while these two quantities specifically measures the amount of a particular species, they are however a qualitative description to indicate the state of the system. By the same token, although the energetic response (E) is given as a quantity 
in "molecules" in the model, it is only a descriptive quality as a marker to track the state of the system and does not have a physical manifestation in physiology. The energetic response (E) refers to those transcriptional signatures that participate in the cellular bioenergetic processes, mainly in the ATP producing pathways (Carre and Singer 2008) and is affected by the transcriptional activities of NF-kB, coupled with the anti-inflammatory cytokine response. Moreover, activated NF-kB, IKK, and IkBa molecules are the summation of activated population of respective species in all macrophages and LPS refers to the total amount of LPS in circulation, both bound and free. Regarding TNF-a, it refers to free TNF-a molecules.

\section{Agent rules and behaviors}

Agents are the main components that follow specific instructions on how they should behave and interact with other agents. Each agent has its own properties that define the type of behavior and interactions that the agent is involved with. Different types of agents are grouped into different classes, i.e., a type of interleukin or stimulus. Some properties are present in many classes, i.e., degradation counter that determines when an agent disappears or die; or location reporter that informs the molecule its position with regard to another molecule. Other properties only pertain to a certain class, i.e., receptor sensitivity that dictates whether binding occurs, or macrophage energetic level that serves as a survival indicator. The "world" is defined by a coordinate system with boundaries that wrap around horizontally and vertically. Macrophages are placed randomly in the world, provided that there is no overlapping between each macrophage. The simulation is computationally intensive in that each macrophage cell alone is composed of more than 400 agents. For the purpose of reducing the computation time for each simulation, a 161 
by 161 world and 4 macrophages were used for the experiments. After experimenting with a range of world sizes, we decided that the selected size was appropriate to accurately generate the dynamic profiles while allowing repeated simulations to run at a desirable pace.

As the model is executed, it performs a list of procedures in an order. The execution is an iterative process where each iteration represents a "tick" or a discrete time point. Each procedure governs the behavior of a specific class of agent; it contains instructions on how an agent should move, whether to bind to a receptor or "bounce" off of the cell membrane, etc. The instructions are conditional (rule) based (if-then) and may involve multiple agents, such as when two molecules bind together, both molecules' parameters change due to the binding, for instance, they now move in the same pattern. Moreover, the instructions are derived from literature regarding relevant mechanisms for LPS activation (Bosshart and Heinzelmann 2007; Du, Poltorak et al. 1999; Monick and Hunninghake 2002), intricate signaling cascades (Delhase, Hayakawa et al. 1999; Hoffmann, Levchenko et al. 2002a; Li and Verma 2002; Mittal, Peak-Chew et al. 2006; Ting and Endy 2002; Zhang, Chan et al. 2005), cytokine network (Gri, Savio et al. 1998; Xing, Jordana et al. 1994; Zhou, Lin et al. 1994) and cell (Th) differentiation (Kidd 2003; Santoni, Pedicini et al. 2008). The specific rules that determine the behavior of the relevant agents are presented in Table 5.3 (see Appendix 5.6).

The movement of LPS molecules is characterized by a random walk routine, namely, each molecule heads to a random direction and moves several steps forward. When one LPS molecule comes in contact with its signaling receptor on the surface of the macrophage, it will have a chance to bind to it. Once bound a molecule will no longer be 
moving freely; it will move in accordance with its counterpart. Such process triggers the production of the proximal inflammation-specific mediator, TNF-a causing a decrease in receptor's sensitivity. Receptor sensitivity determines the probability by which one LPS molecule will bind to its endotoxin receptor. Bound LPS molecules are degraded shortly, freeing up the receptor. Molecules of TNF-a also move randomly where they can diffuse into the cell (move past the cell membrane agents) and bind to their appropriate receptors either from the cytoplasm or from outside of the membrane. The activated receptor will trigger downstream a signal transduction cascade that stimulates IKK activity. Activated IKKs move randomly inside the cytosol while they are not capable in moving past the cell membrane agents or enter the nucleus region. They activate the NF-kB complex by dissociating the bound between NF-kB and its inhibitor, IkBa. This is achieved through the phosphorylation of the inhibitory protein IkBa where dissociated $\mathrm{IkBa}$ is therefore ubiquitinated and degraded by the proteasome. Activated NF-kB then moves into the nucleus region initiating the transcriptional machinery program which up-regulates the transcription of IkBa, of pro-inflammatory cytokines $(\mathrm{P})$ followed by a decrease in the energetic state of the macrophage. Activated IkBas are capable of moving into the nucleus, binding to activated NF-kB molecules, and deactivate them, as they retrieve nuclear concentrations of NF-kB by forming an inactive complex in the cytoplasmic region.

The pro-inflammatory $(\mathrm{P})$ agents are limited by cell membranes and their presence excites the production of anti-inflammatory cytokines (A) and the migration of undifferentiated T-helper cells (Th0) which are not present under conditions of no infec7tious challenge. However, the secretion of pro-inflammatory $(\mathrm{P})$ molecules by 
macrophages during the progression of the inflammatory reaction induces Th0 cells to enter the "virtual world". This event signifies the migration of cytokines into the spleen, within which the differentiation of Th0 cells takes place. In this model the role of Thelper cells is to regulate the feedback loops associated with pro-(P) and antiinflammatory (A) mediators. Further, the binding of (A) molecules with their signaling receptor will stimulate an energy expenditure causing nearby bound TNF-a to degrade faster. In addition to Th0 cell type under conditions of an abundance of bound antiinflammatory (A) cytokines with their receptors it will morph into a Th2 cell. If it happens that the presence of $(\mathrm{P})$ molecules on the surface receptors to outweigh the (A) response, then Th0 will become of Th1 type which potentiates the pro-inflammatory response. Conversely, Th2 cell type response potentiates the secretion of antiinflammatory cytokines (A).

In order to facilitate the translation from literature evidence into a programming language, it is necessary to provide a feasible framework that integrates disparate research into a conceptually valid scheme, taken into account the abstractness and limitations of the model. Some of the goals are outlined in literature (An 2006) but in this model, we place emphasis on the potential mechanisms that drive complex responses identifying essential elements of the probed response.

\section{Model calibration and validation}

Due to the inherent stochasticity of the ABM development, calibration is oftentimes performed on a trial and error basis. This process involves generating multiple sets of results by systematically varying the model parameters at each set. Also known as "parameter sweeping", this process allows us to explore the possible behaviors of the 
model and determine which parameters will engender the patterns that best represent the behavior of interest. For instance, we first examine the signaling agents that will have the most leverage on repressing the inflammatory response. After each simulation, we adjust the parameters such as the production rate of P and TNF-a, the movement speed of LPS, or the probability that an interaction will occur between two colliding signaling molecules. These parameter values were manipulated so that the simulations lie in qualitative agreement with the self-limited inflammatory response. This implies that from among the multiple runs we select those that can effectively reproduce dynamic profiles associated with the successful elimination of the inflammatory stimulus within the first $2 \mathrm{hr}$ post-endotoxin administration while followed by a subsequent transcriptional resolution within $24 \mathrm{hr}$.

We define the parameters that can produce the self-limited profile as a basis set and based on this set we simulate the LPS dosage dependent responses. A set of parameters is considered satisfactory if the model is capable of simulating the dynamics of a selflimited inflammatory response (resolution within $24 \mathrm{hr}$ post-LPS administration) as well as successfully generating the series of unconstrained (non-linear) responses as previously discussed in this paper. The results of the simulations are compared on a qualitative manner with our prior equation-based host response models as shown in Figure 5.24 and Figure 5.25 (dashed lines). While comparing the output of ABM with the output of the ODE, it should be noted that both modeling approaches are not characterized by the same network topology. Specifically, in the proposed ABM additional inflammatory mediators (molecules, cells) are considered when compared to the ODE model which may account for the observed variations in the simulated 
responses of the two modeling frameworks. However, albeit different in network topology, the two modeling frameworks predict responses (i.e. P, A, E) that lie in a good qualitative agreement.

This ABM is developed using NetLogo (Center for Connected Learning and Computer-Based Modeling, Northwestern University, Evanston, IL), a freeware that constructs agent based models.

\subsubsection{Qualitative assessment of the model}

A self-limited inflammatory response to the endotoxin stimulus corresponds to resolved dynamic profiles for all the elements constituting the model. The objective was to produce the dynamic profiles of a successful inflammatory resolution as shown in Figure 5.23 that qualitatively agreed with the previously models using a deterministic approach (Foteinou, Calvano et al. 2009a, b). While the inflammatory stimulus, namely LPS agents were successfully cleared within $1 \mathrm{~h}$, the activation of anti-inflammatory cytokines expedited the attenuation of the early pro-inflammatory cytokine TNF-a with subsequent termination of the pro-inflammatory signaling cascade. Inevitably there is a level of abstraction that needs to be considered when representing molecular reactions as discrete events that follow somewhat arbitrary rules. The correctness of the model was evaluated based on its ability to qualitatively predict the uncontrolled responses as below. 

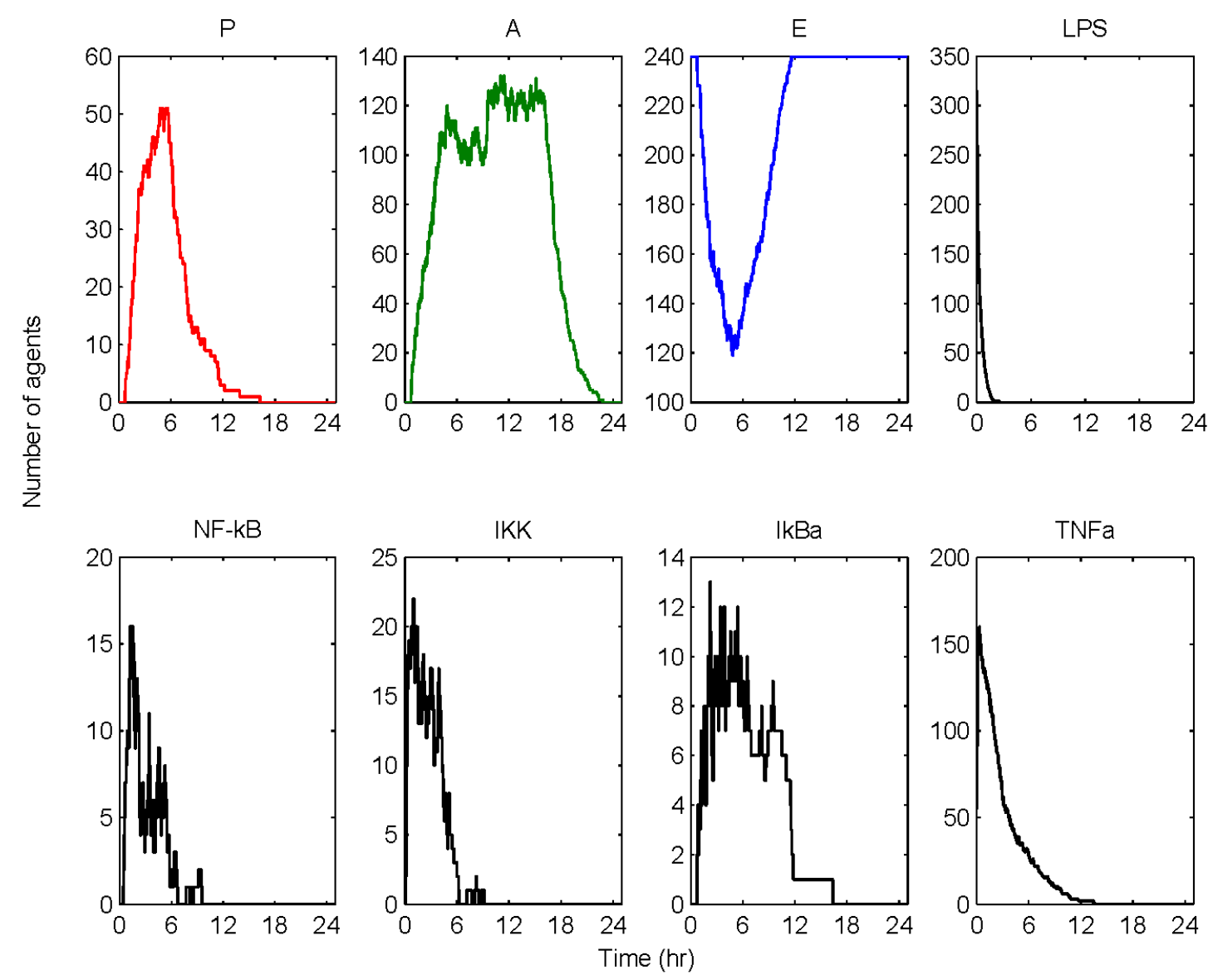

Figure 5.23: A self-limited inflammatory response ( $\operatorname{LPS}(0)=350$ units). Temporal profiles of essential components that constitute the agent based model resolved within $24 \mathrm{hr}$. 
The validity of our approach will be demonstrated through its potential to reproduce biologically relevant scenarios indicative of the non-linear dynamics of systemic inflammation described as the following scenarios: (1) a persistent infectious response where the inflammatory stimulus was not eliminated, leading to an aberrant inflammatory response, (2) a persistent non-infectious inflammatory response that can be elicited under high concentrations of the inflammatory stimulus, causing an inflammatory insult that can disturb the dynamics of the host response leading to an unconstrained inflammatory response; and finally, (3) two scenarios associated with endotoxin tolerance and potentiation effects followed by perturbations in the regulatory (NF-kB) signaling module.

\section{$\underline{\text { Implications of increased insult }}$}

High concentrations of LPS, corresponding to an increase in the strength of the inflammatory insult, can be responsible for the amplification of the host immune response (Munford 2006). This event is followed by a dysregulation in host defense intrinsic dynamics leading to an unconstrained inflammatory response even after the circulating levels of LPS have been cleared. The model predicted the situation where the initial levels of LPS are increased in Figure 5.24. We observed that when the concentration of the inflammatory stimulus exceeded a critical threshold, the inflammatory response did not abate. Such a response is characterized by overwhelming production of the pro-inflammatory instigator, TNF-a, which amplifies the activity of NF-kB. In particular, high LPS concentration potentiates the secretion of proinflammatory mediators $(\mathrm{P})$ which in turn may increase the probability of Th0 cells to differentiate into Th1 cells rather than into Th2 cells. Additionally Th1 cells further 
increase $(\mathrm{P})$ population; thus disturbing the balance between Th1/Th2 accounted for the progression of an unconstrained inflammatory response.
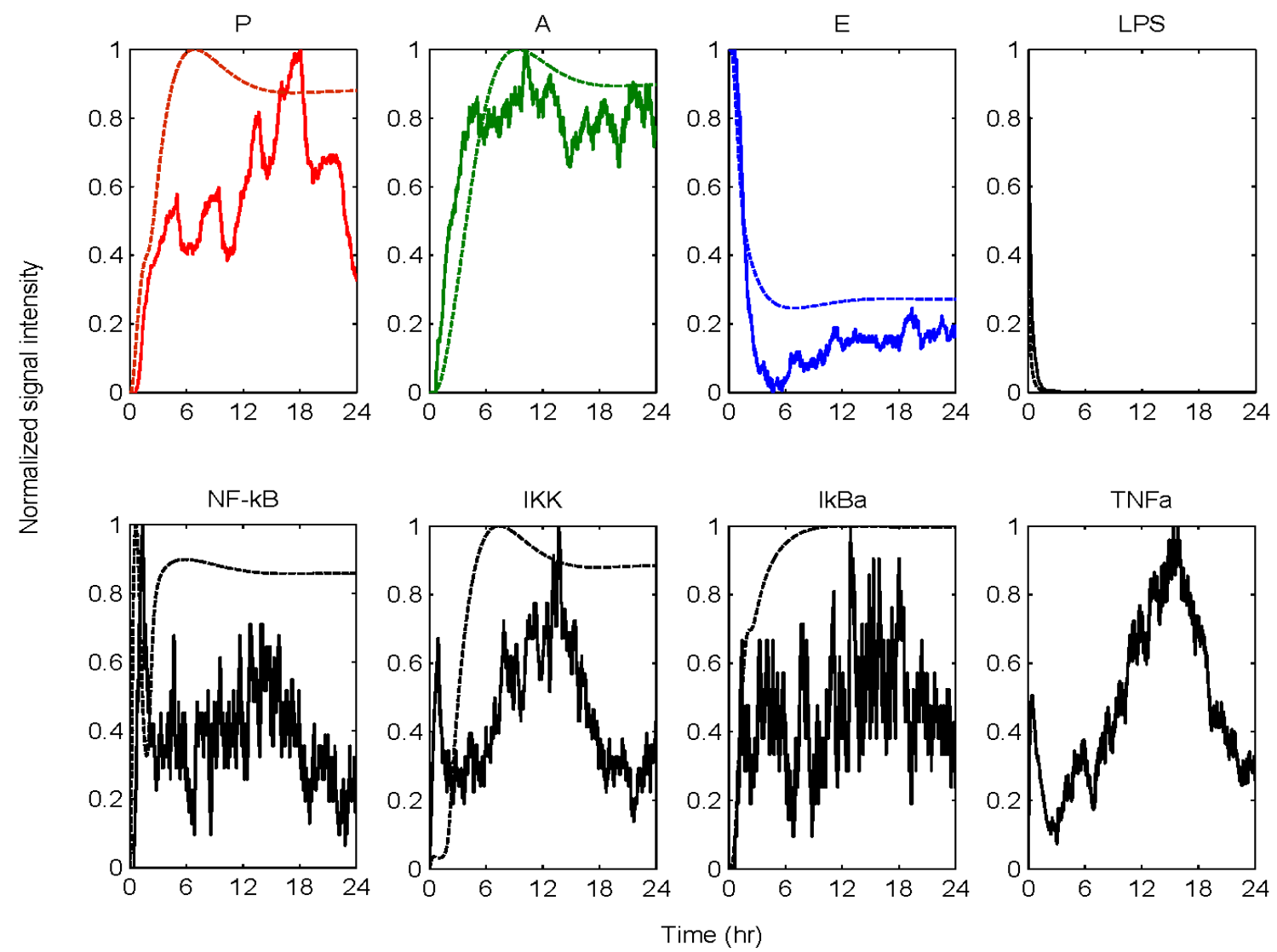

Figure 5.24: Temporal responses of an unresolved inflammatory response due to high LPS concentration. A high concentration of LPS (LPS $(0)=750)$ can cause a malfunction in the dynamics of the host leading to an exacerbated inflammatory response (solid lines). Dashed lines refer to the implications of high concentration of LPS as simulated by our deterministic (ODE) approach. For the purpose of comparing the simulated output between the ABM and the ODE model, all responses are normalized so that numerically they range between $(0,1)$. 


\section{Malfunction in LPS clearance rate}

An acute pro-inflammatory cytokine "burst" results from intravenous administration of high concentration of LPS into the system of healthy subjects. The subsequent effect is associated with the host's inability to resolve the inflammatory reaction followed by the persistent infectious challenge (unsuccessful clearance of endotoxin) (Xing, Jordana et al. 1994; Zhou, Lin et al. 1994). Accordingly, the prolonged exposure of the system to bacterial infection leads to a significant down-regulation of the endotoxin signaling receptor which further accounts for a slower decay rate causing a dysregulation in the phagocytic capabilities of macrophages (Nomura, Akashi et al. 2000).

The relevant agent rule that captured such scenario was the "sensitivity" parameter of the endotoxin signaling receptors. As these receptors become saturated during the presence of high amounts of endotoxin, the sensitivity parameter decreases which thereby influences the probability of LPS receptors to be occupied with LPS molecules. During an overwhelming endotoxin challenge, the LPS receptors eventually lose their capability to form additional complexes with LPS, and therefore the LPS agents remain in the system. Although decreased degradation of LPS is not associated with a distinct, welldefined, clinical condition, it is possible that this phenomenon may exist. It is known that triglyceride-rich lipoproteins bind to LPS and that these complexes are cleared by binding to lipoprotein receptors (Du, Poltorak et al. 1999). Furthermore, these receptors are abundant in the liver where $\sim 70 \%$ of lipoproteins are cleared from the circulation. Such malfunction in LPS clearance rate was simulated in Figure 5.25. 

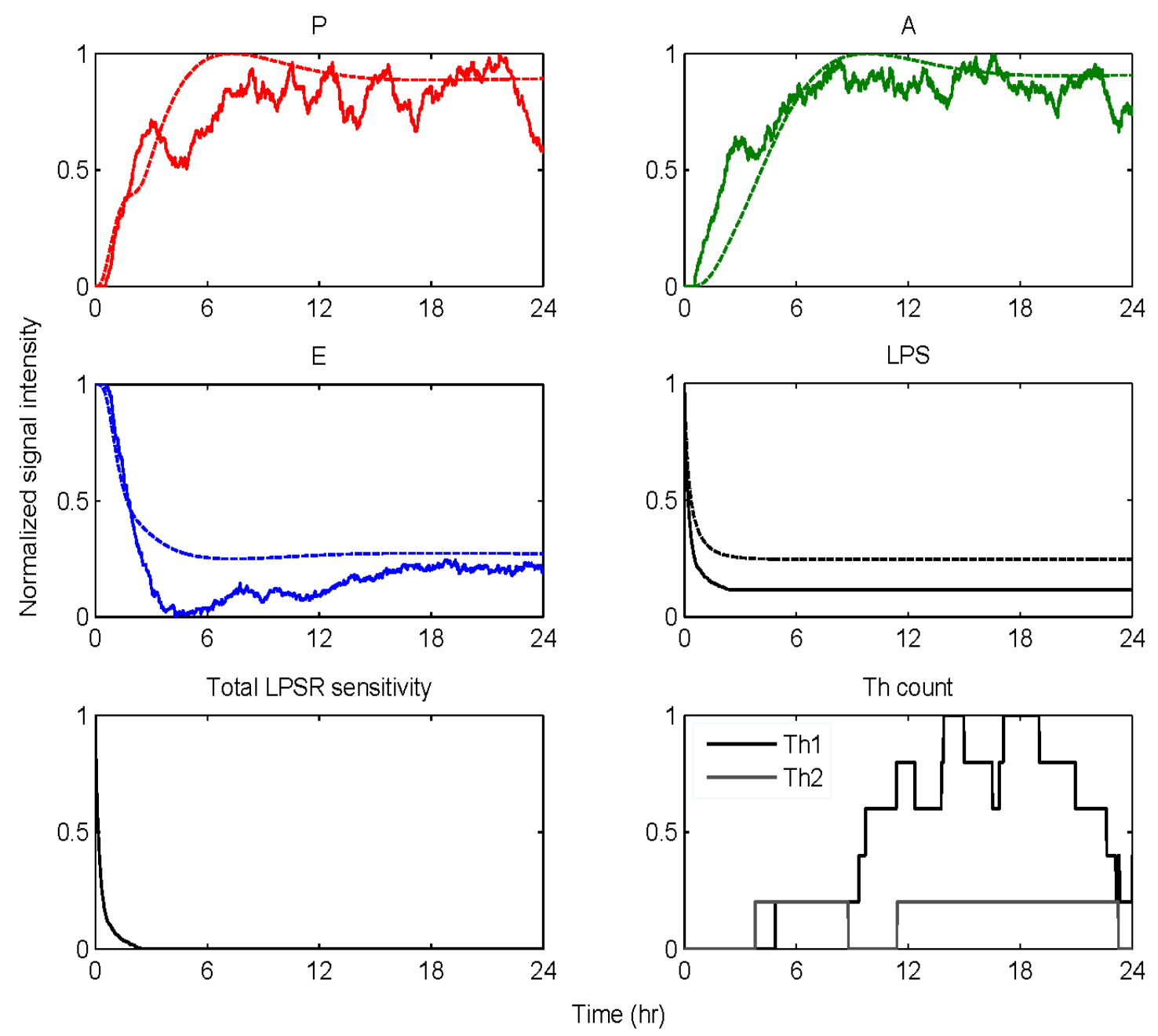

Figure 5.25: Temporal responses in a persistent infectious inflammatory response. Solid lines correspond to $\operatorname{LPS}(\mathrm{t}=0 \mathrm{hr})=1000$ which accounts for a prolonged inflammatory activity causing a malfunction in LPS clearance rate. Dashed lines refer to equation-based model predictions for the case of a persistent infectious challenge which can be achieved by manipulating the first order degradation rate of LPS as discussed in the deterministic approach (Section 5.3). The output of both modeling approaches is normalized so that numerically it ranges between $(0,1)$. 
Similar to the progression of the increased insult scenario as shown in Figure 5.24, the progression of a persistent infectious response (Figure 5.25) was simulated due to the activation of the feedforward loop regarding the activation of IKK which drives downstream an aberrant transcriptional activity of NF-kB and thereby affecting the transcriptional rate of the critical pro-inflammatory mediators, i.e. TNF-a. The secretion of TNF-a further amplified the activity of NF-kB through the critical IKK node (Sakurai, Suzuki et al. 2003). These interactions perturb the dynamics associated with the energetic state of the system. Furthermore, we speculated that a switch-like rule related to the energetic state of the cell can be responsible for the disturbance of the homeostatic production of anti-inflammatory mediators. Such rule has been implemented in the ABM framework in that when the energetic state is below $25 \%$ of its original value the production of the anti-inflammatory mediators should increase.

\section{Endotoxin hypo-responsiveness}

The pre-exposure of the host to controlled levels of inflammatory agents affects the eventual fate of the response. It has been observed that repeated doses of endotoxin insult might lead to a less vigorous innate immune response (Fitting, Dhawan et al. 2004). Such an effect can reverse the lethal outcome of a high dose of the inflammatory stimulus. That is to say, in spite of the potent efficacy of LPS, if the system is pre-exposed to lower sublethal doses of LPS then this induces an acquired state of resistance to a subsequent endotoxin challenge (Cook 1998). This phenomenon, known as endotoxin hyporesponsiveness is a multifactorial problem that can be associated with decreased TLR signaling by proteins that negatively regulate LPS-induced inflammatory responses (Cook, Pisetsky et al. 2004). 
From a modeling standpoint, small dose of LPS is administered 8 hours prior to the main endotoxin insult. Such perturbation modulates the dynamic profiles of both proinflammatory and anti-inflammatory mediators as well as the energetic state of the macrophage populations towards resolution within 24 hours. The endotoxin hyporesponsiveness was simulated in Figure 5.26 where pre-existing infection caused a profound reduction in cells' capacity to respond to the main (high) endotoxin challenge. There were no agent rules that specified the time interval between the injections that would yield the emergent attenuated response. From a biological standpoint the prior inflammatory insult desensitizes the endotoxin signaling receptors in a manner that these receptors become less sensitive to the subsequent infectious challenge and therefore the cells have enough time to mitigate the endotoxin challenge and resolve the inflammatory reaction. 

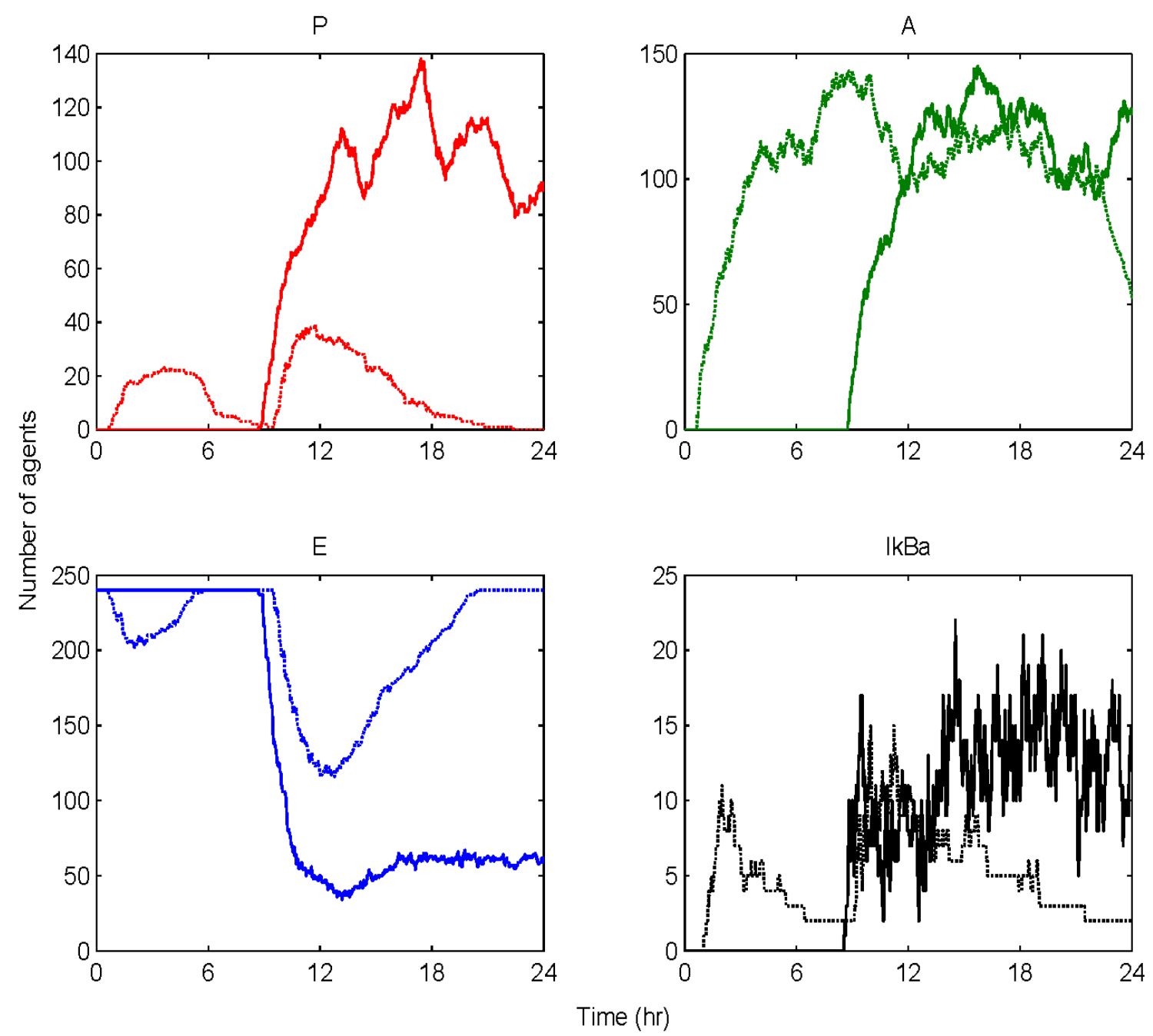

Figure 5.26: Endotoxin tolerance scenario. Pre-existing infection might cause a profound reduction in cell's capacity (hypo-responsiveness) to respond in the main endotoxin challenge. Solid line: $\operatorname{LPS}(\mathrm{t}=\mathrm{hr})$ $=750$. Dotted line: $\operatorname{LPS}(\mathrm{t}=0 \mathrm{hr})=100 \& \operatorname{LPS}(\mathrm{t}=8 \mathrm{hr})=650$. 


\section{"Lethal" potentiation}

Endotoxin hypo-responsiveness is associated with an emergent acquired dynamic state of the system that modulates the response of the system not to respond rigorously to the primary endotoxin challenge. On the other hand, the successive administration of sublethal doses of endotoxin can potentiate the system in that, because of the lack of an acquired state in the dynamics of the system, such an insult may dysregulate the host response dynamics leading to an exacerbated inflammation that cannot resolve. Thus, based on our agent-based model we further explore the behavior of the system when it is either pre-exposed to lower levels of endotoxin for "adequate" time as well as when the system has not manifested its "dynamic memory" to tolerate the second endotoxin challenge (Murai, Nakagawa et al. 1996).

In particular, we simulate such a case administering at $\mathrm{t}=0 \mathrm{hr}$ low dose of endotoxin which is shortly followed within $2 \mathrm{hr}$ by another "sub-lethal" insult. From a modeling standpoint, this short time interval was characterized by the accumulation of both proinflammatory (P) and anti-inflammatory (A) mediators. The response was exaggerated under conditions of the second endotoxin stimulation due to the priming of various inflammation-specific intracellular signaling molecules which further propagated the inflammatory reaction to nearby cells/agents. The effect of this lethal potentiation scenario was demonstrated in Figure 5.27. Additionally, if the second dosage was administered when the inflammatory mediators are diminishing, then the effect is less prominent due to both the lack of cytokines and the receptor desensitization which occurs due to pre-existing infection. 

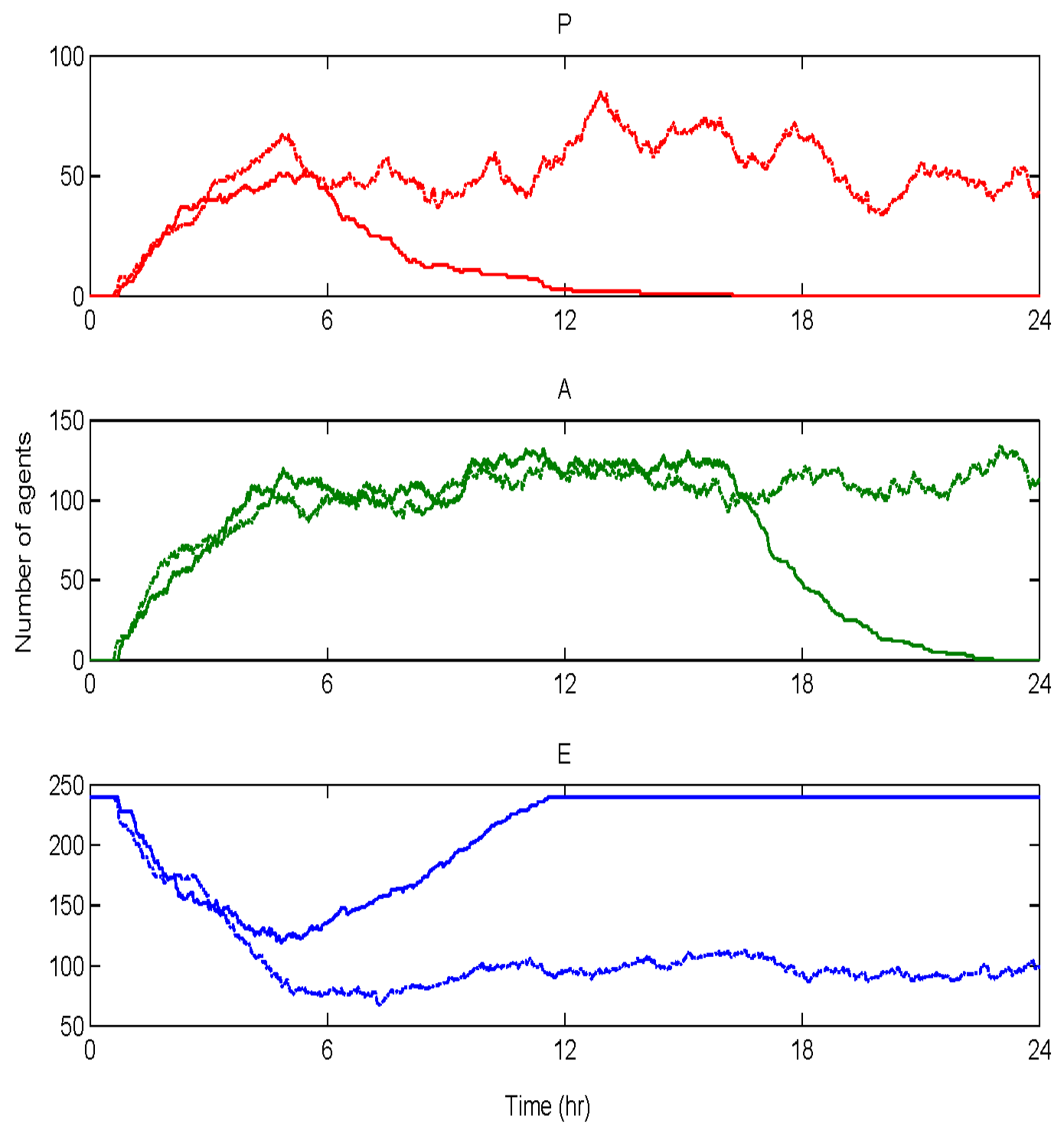

Figure 5.27: Lethal potentiation. Successive administration of small doses of endotoxin can lead to an unresolved inflammatory response. Solid line: $\operatorname{LPS}(\mathrm{t}=0 \mathrm{hr})=350$. Dotted line: $\operatorname{LPS}(\mathrm{t}=0 \mathrm{hr})=100 \& \operatorname{LPS}(\mathrm{t}$ $=2 \mathrm{hr})=250$. 
Modulation in the dynamics of NF-kB signaling module

Another mode of perturbation of the underlying dynamics of the probed system was related to the presence of a "prior" insult that coupled with the LPS stimulus. It accounted for an increased production of pro-inflammatory mediators as shown in Figure 5.28(left panel). Such a sustained pro-inflammatory signaling was possible to deregulate the NF$\mathrm{kB}$ signaling module and led to a persistent NF-kB activity (Yamakawa, Eguchi et al. 1999). The elevated NF-kB activity implied that the nuclear concentration of NF-kB cannot be further constrained by its primary inhibitor, IkBa and eventually settled to a steady state far away from their equilibrium (homeostasis). We simulated this scenario by pre-conditioning the system with low-dose of TNF-a. Since TNF-a is a potent inflammatory instigator that stimulates IKK activity it can perturb the behavior of the system towards an unbalanced immune response. Clinically, such an increased rate in the production of pro-inflammatory mediators might be the outcome of a surgical trauma followed by bacterial infection, a so called two hit scenario (Romascin, Foster et al. 1998).

We have demonstrated the ability of our model to simulate the trajectory of an unconstrained inflammatory response. Further, the potential of the proposed model was also demonstrated through its capability to respond to an intervention strategy that intended to modulate the dynamics in favor of a balanced immune response. In Figure 5.28(right panel) the effectiveness of a molecule that inhibited IKK activity (IKKinhibitor) was simulated. From a biological standpoint, these molecules diffuse into the cytoplasm and bind to IKK triggering its deactivation. This process directly competed with the activation of the NF-kB complex through IKK and therefore attenuation in the 
pro-inflammatory response was observed. As such, despite the implications of high LPS concentration, the dynamics were reversed towards homeostasis. Qualitatively, this result agreed with experimental data that documented the potential of IKK inhibitors in treating inflammatory disorders (MacMaster, Dambach et al. 2003).
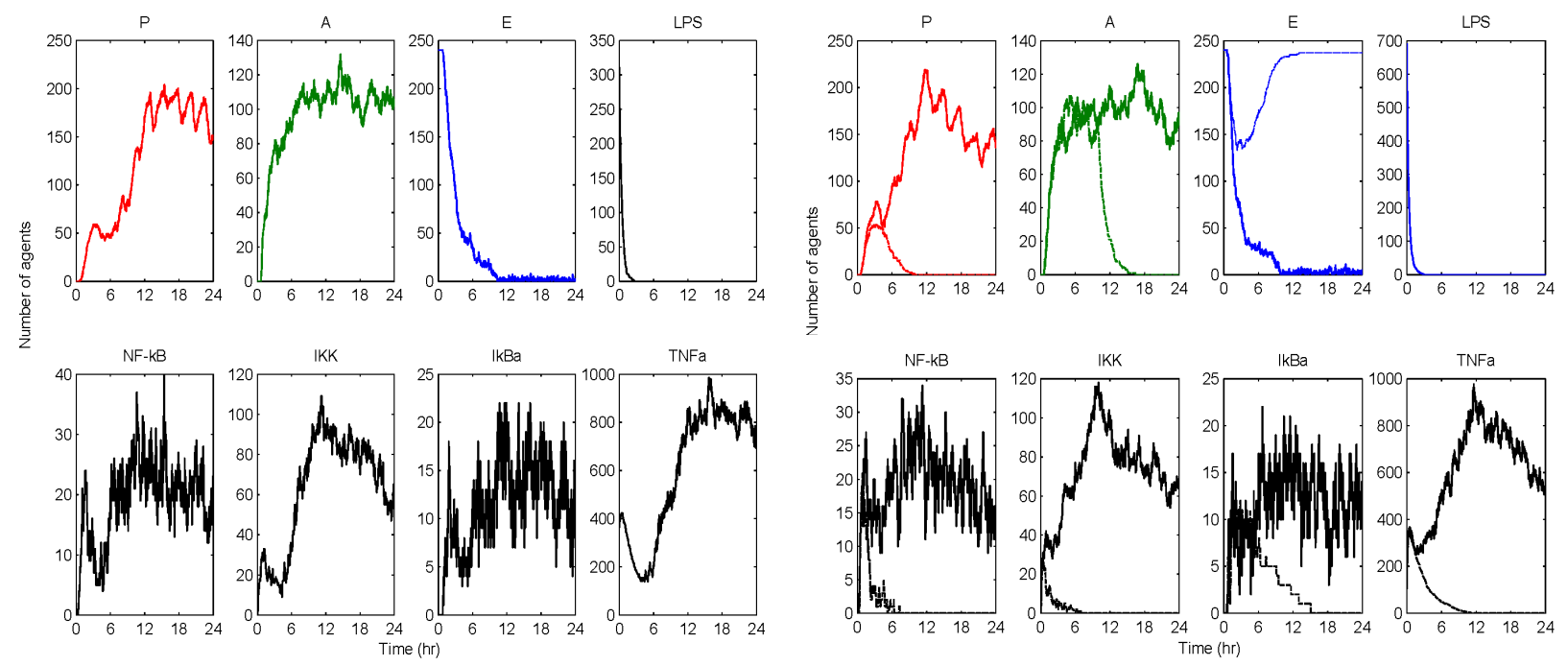

Figure 5.28: (left) Pre-existence of inflammatory mediators (TNF-a) may enhance abnormally the intracellular signaling amplifying IKK activity. Such response leads to an aberrant inflammatory response which cannot be counter-regulated by the anti-inflammatory arms of the system. Such a mode of dysregulation is simulated by concomitant exposure of the system to TNF-a and bacterial infection (LPS): LPS( $\mathrm{t}=0 \mathrm{hr})=350 \& \mathrm{TNF}-\mathrm{a}(\mathrm{t}=0 \mathrm{hr})$ =300; (right) Exploring the effect of an intervention (anti-inflammatory) strategy that inhibits IKK activity. Such scenario is simulated by administering $\operatorname{LPS}(\mathrm{t}=0 \mathrm{hr})=750$ and IKK inhibitors, IKK inhibitors $(\mathrm{t}=0 \mathrm{hr})=400$ (dashed lines). 
The proposed ABM model exhibited a bistable behavior which implied the coexistence of two steady states. Physiologically, such dynamics would reflect either a successful inflammatory resolution or the progression of a systemic inflammatory response syndrome. Such bistability is an essential characteristic of the non-linear dynamics of inflammation as suggested from various animal studies [36]. In an attempt to simulate the bistable behavior, a "switch" in the agent rules was employed. A switch was defined as a conditional procedure under which the output could diverge into different states based on a current set of inputs. In our simulation the switch was the production rate of TNF-a with regard to the energetic state of the macrophage. As the current energy value become lower than a certain threshold, the production of TNF-a via the transcription factor NF-kB was amplified, activating the switch. The rationale behind this rule was predicated upon the hypothesis that the activation of NF-kB, followed by the production of $(\mathrm{P})$ response ultimately decrease the expression of genes that are involved in bio-energetic cellular processes (Protti and Singer 2007). On the other hand the number of anti-inflammatory (A) molecules raised the energy level and drove the cells to the "healthy" state, deactivating the switch. Many switch-like phenomena have been observed in biological systems (Ramakrishnan and Bhalla 2008).

In summary, an agent based modeling framework is proposed as an alternative modeling approach to study the complex, non-linear dynamics of acute human inflammation. Agent based models offer a promising approach in that they can express the dynamics through intuitive multiple interactions between the agents over time. A well known feature of ABMs is their ability to generate surprisingly complex and emergent behavior from very simple rules. Although they are abstract and have their own 
limitations (i.e. computationally intensive, difficult to be quantitatively calibrated), ABMs facilitate the translation from the results of basic science experiments into agent rules that allow for heterogeneous responses based on local conditions. We therefore proposed an agent based model that couples critical aspects of the host response to endotoxin. Predicated upon our prior research effort where a deterministic approach has been taken to couple extracellular signals and intricate signaling cascades with the transcriptional response level, the work discussed in this study explored the potential of an agent based modeling approach to improve our understanding of how a system gives rise to a response through its interacting molecules (or cells). The recognition that both approaches (EBM and $\mathrm{ABM}$ ) have their advantages and limitations has placed emphasis on cross-platform validation where some processes are simulated discretely while other processes are handled in a continuous simulation (Kiehl, Mattheyses et al. 2004). It is important to realize that such multi-modal approaches are complementary and ideally both would be used to provide a mathematical characterization of a complex dynamical system. 


\subsection{Appendix to the agent-based model of acute human inflammation}

Table 5.3: List of agent-based rules

\begin{tabular}{|c|c|}
\hline Agents & Agent rules \\
\hline \multirow[t]{3}{*}{ Macrophages } & Produce 1 unit of IKK every 5 ticks \\
\hline & Move towards the direction that has the highest LPS count within $5+$ cell radius \\
\hline & Bind free LPS molecules with unoccupied receptors \\
\hline \multirow[t]{6}{*}{ IKK } & Activated by the formed TNF-TNFR complex \\
\hline & $70 \%$ chance to bind to inactive NF-kB \\
\hline & Dissociate from the complex after 10 ticks \\
\hline & Stimulates Nf- $\mathrm{kB}, \mathrm{IkBa}$ is ubiquitinated \\
\hline & Deactivated by activated IkBa as a result of the transcriptional activity of NF-kB \\
\hline & Degrades after a random of 1 to 799 ticks \\
\hline NF-kB & $\begin{array}{l}\text { If activated and translocates to the nucleus, asks the macrophage to produce } 1 \mathrm{IkBa} \text { every } 10 \\
\text { ticks, } 1 \text { unit of IL- } 12 \text { with } 80 \% \text { chance and } 1 \text { unit of TNF-a }\end{array}$ \\
\hline \multirow[t]{2}{*}{$\mathrm{IkBa}$} & If activated, seek out activated NF-kB within radius of 1 \\
\hline & $\begin{array}{l}\text { Bind to any activated NF-kB, form a complex and both members of the complex become } \\
\text { inactivated }\end{array}$ \\
\hline \multirow[t]{2}{*}{ Receptors } & $\begin{array}{l}\text { TLR4 (LPS receptors) become activated when bind to LPS molecules. If activated, then } \\
\text { produce } 1 \text { unit of TNF-a every } 100 \text { clicks }\end{array}$ \\
\hline & IL-4 and IL-12 receptors receive their respective targets (receptors) \\
\hline \multirow[t]{2}{*}{ IL-4 } & In the presence of free IL-12, 3 units of IL- 4 are produced by $86 \%$ for every 1 to 5 clicks \\
\hline & $\begin{array}{l}\text { Bind to IL- } 4 \text { receptors on Th- } 0 \text { cells or macrophages. On macrophages, the binding rate } \\
\text { increases the energetic level of the macrophage by } 1\end{array}$ \\
\hline \multirow[t]{2}{*}{ IL-12 } & Produced by macrophages and Th-1 cells \\
\hline & Bind to IL-12 receptors on Th- 0 directing the differentiation towards either Th- 1 orTh- 2 \\
\hline Th cells & $\begin{array}{l}\text { Th- } 0 \text { cells count the unit of interleukins on its surface receptors. Once the number of } \\
\text { interleukins reaches } 25 \text {, then differentiate into either Th- } 1 \text { or Th- } 2 \text {. If more IL- } 12 \text { molecules } \\
\text { are present than IL- } 4 \text {, then become Th- } 1 \text {. Otherwise, it becomes a Th- } 2 \text { type }\end{array}$ \\
\hline \multirow[t]{6}{*}{ LPS } & Frequency of movement: 800 times more frequent than other cellular agents \\
\hline & Collides with LPS receptors on the surface of macrophages \\
\hline & Activates the receptor while the "sensitivity value" decreases by 1 \\
\hline & $\begin{array}{l}\text { A successful binding occurs if a random value between } 1 \text { and the maximum value of } \\
\text { sensitivity (which equals to 5) is less than the current sensitivity }\end{array}$ \\
\hline & Activated endotoxin receptors produce 1 unit of TNF-a \\
\hline & If binds to its receptor, then degrade 1 to 2 ticks \\
\hline \multirow[t]{4}{*}{ TNF-a } & Start with 600 ticks \\
\hline & Binds to TNFR on the surface of macrophages \\
\hline & Amplifies intracellular IKK activity \\
\hline & $\begin{array}{l}\text { If it binds to a receptor, then degrade in } 50 \text { clicks or in } 200 \text { clicks if there is nearby bound IL- } \\
\text { 4. Degrades by } 1 \text { click naturally }\end{array}$ \\
\hline \multirow[t]{2}{*}{$\begin{array}{l}\text { Movement } \\
\text { restrictions }\end{array}$} & $\begin{array}{l}\text { Intracellular molecules i.e. NF-kB, IkBa, IKK are confined inside the agents of the plasma } \\
\text { membrane. Only activated NF-kB and IkBa can enter the nucleus. Before these agents } \\
\text { perform any rule based movement, a check is made whether they are facing the membrane, } \\
\text { other molecules or the nucleus. If the destination is inaccessible, then they will face another } \\
\text { direction until the next move is achieved. Their positions are further updated as the } \\
\text { macrophage moves }\end{array}$ \\
\hline & $\begin{array}{l}\text { Extracellular molecules cannot enter the nucleus have similar restrictions with the plasma } \\
\text { membrane agents }\end{array}$ \\
\hline
\end{tabular}




\subsubsection{List of agents}

\begin{tabular}{|l|l|}
\hline Cell types & Macrophage, Th-0, Th-1, Th-2 \\
\hline Stimulus, mediator & LPS, IL-12, IL-4, TNF-a \\
\hline Receptors & TLR4, IL-12R, IL-4R, TNFR \\
\hline Intracellular signaling molecules & IKK, NF-kB, IkBa \\
\hline Cellular component & Plasma membrane, nucleus \\
\hline
\end{tabular}




\section{Chapter 6}

\section{Multiscale Models of Human Endotoxemia}

Severe injury and infection are associated with autonomic dysfunction. The realization that a dysregulation in autonomic function may predispose a host to excessive inflammatory processes has renewed interest in understanding the role of central nervous system (CNS) in modulating systemic inflammatory processes. Assessment of heart rate variability (HRV) has been used to evaluate systemic abnormalities and as a predictor of the severity of illness. Dissecting the relevance of neuro-immunomodulation in controlling inflammatory processes requires an understanding of the multiscale interplay between CNS and the immune response. A vital enabler in that respect is the development of a systems-based approach that integrates data across multiple scales, and models the emerging host response as the outcome of interactions of critical modules.

Based on our prior work as discussed in the previous chapters, a cellular level physicochemical host response model is used as template for connecting extracellular signals and intracellular signaling cascades eventually leading to the emergent transcriptional dynamics (Foteinou, Calvano et al. 2007; Foteinou, Calvano et al. 2009a, b). However, one of the simplifying hypotheses made in this model is that the immunomodulatory role of hormonal influences, including endogenous cortisol, is not explicitly incorporated. It is now well established that the human response to endotoxin evokes both leukocyte transcriptional alterations and a neuro-endocrine response characteristic of acute injury (Lin and Lowry 1998). Accordingly, a rise in circulating endocrine hormones is manifested 2-4 hrs following endotoxin administration (Lowry 
2005). Driven by the premise that a characteristically enhanced endocrine hormone profile is elicited during the early-phase response to endotoxin injury, essential modules associated with the bi-directional communication between the immune response and the neuro-endocrine axis (HPA, SNS) are considered. Moreover, diminished heart rate variability in association with a hyperdynamic state, as a component of autonomic dysfunction, is induced by low-dose endotoxin to human subjects (Alvarez, Katsamanis Karavidas et al. 2007; Jan, Coyle et al. 2009). Thus, the acute systemic inflammatory condition mediated by endotoxin administration in healthy volunteers elicits a complex network of multiscale interactions between the immune and the central nervous system (CNS) which is essential for maintaining homeostasis.

In this chapter, multiscale models of human endotoxemia are developed that integrate regulatory processes across the host from the cellular to the systemic level. Of particular relevance of this chapter are human data associated with plasma concentrations of neuroendocrine hormones including cortisol and catecholamines. Further, clinical measurements of heart rate and parameters of heart rate variability are also incorporated to assess cardiovascular abnormalities and systemic decomplexification manifested as very early diminution in parameters of $\mathrm{HRV}$ and/or increased heart rate (HR). It is therefore the goal of this research to demonstrate the feasibility of a relevant human inflammation model that bridges the initiating signal and phenotypic expressions (HR, HRV) through semi-mechanistic based host response models that include transcriptional dynamics, signaling cascades and hormonal components that should not be viewed as distinct functional domains. Such a modeling approach could potentially provide 
invaluable insights into how disruption within these compartments contributes to morbidity and mortality in severely stressed patients.

\subsection{Modeling neuro-endocrine immune system interactions}

The primary stress response pathway by which the CNS regulates the immune system is the hypothalamic-pituitary adrenal axis (HPA) through the production of glucocorticoids (cortisol in primates; corticosterone in most rodents). The HPA, as one of the peripheral limbs of the stress system, responds to the pro-inflammatory cytokines produced by immune-mediated inflammatory reactions by releasing cortisol that inhibits proinflammatory cytokine expression (Chrousos 1995; Webster, Tonelli et al. 2002). In order to mathematically describe the dynamics of cortisol, a joint PK model (Mager, Lin et al. $2003)$ is employed as shown in the following equations (6.1) - (6.3).

$$
\begin{gathered}
\frac{\mathrm{dF}}{\mathrm{dt}}=\mathrm{W}_{\mathrm{F}_{\mathrm{ex}}} \cdot \mathrm{R}_{\mathrm{in}, \mathrm{F}}+\mathrm{K}_{\mathrm{in}, \mathrm{F}} \cdot\left(1+\mathrm{H}_{\mathrm{F}, \mathrm{P}}\right)-\mathrm{K}_{\mathrm{out}, \mathrm{F}} \cdot \mathrm{F} \\
\mathrm{H}_{\mathrm{F}_{\mathrm{P}}}=\mathrm{k}_{\mathrm{F}, \mathrm{P}} \cdot \mathrm{P} \\
\mathrm{W}_{\mathrm{F}_{\mathrm{ex}}}=\left\{\begin{array}{l}
1, \text { exogenous hormone } \\
0, \text { elsewhere }
\end{array}\right.
\end{gathered}
$$

Total plasma cortisol concentrations are defined as the additive effect between endogenous and exogenous cortisol. Upon inflammatory stimulation, the rate of change of total cortisol concentration (F), Eq. (6.1), is described by a zero order production rate $\left(\mathrm{K}_{\mathrm{in}, \mathrm{F}}\right)$ stimulated by the pro-inflammatory response $(\mathrm{P})$, through the activation function $\left(\mathrm{H}_{\mathrm{F}, \mathrm{P}}\right)$, Eq. (6.2) and a $1^{\text {st }}$ order elimination rate constant $\left(\mathrm{K}_{\mathrm{out}, \mathrm{F}}\right)$. Further, the contribution of exogenous cortisol upon total cortisol concentrations is assessed through the stimulatory parameter $\left(\mathrm{R}_{\mathrm{in}, \mathrm{F}}\right)$ which is active based on the binary variable $\left(\mathrm{w}_{\mathrm{Fex}}\right)$. 
Given a quantification of the cortisol dynamics, equations (5.35) - (5.36), the influence of cortisol on the host response to endotoxin can be simulated. However, it should be noted that these equations, as outlined in the original analysis (Jin, Almon et al. 2003), describe receptor/gene-mediated corticosteroid effects based on results from an in vivo adrenalectomized model and therefore the baseline value of plasma cortisol is zero. However, in our model the baseline value of cortisol (F) equals to one (Note that it represents the concentration of cortisol relative to the measured response at $\mathrm{t}=0 \mathrm{hr}$ ) and Eq. (5.35) is modified as follows:

$$
\begin{gathered}
\frac{\mathrm{dR}_{\mathrm{F}}}{\mathrm{dt}}=\mathrm{k}_{\mathrm{syn}_{-} \mathrm{R}} \cdot \mathrm{R}_{\mathrm{m}}+\mathrm{r}_{\mathrm{f}} \cdot \mathrm{k}_{\mathrm{re}} \cdot \mathrm{FR}(\mathrm{N})-\mathrm{k}_{\mathrm{on}} \cdot(\mathrm{F}-1) \cdot \mathrm{R}_{\mathrm{F}}-\mathrm{k}_{\mathrm{dgr}_{-} \mathrm{R}} \cdot \mathrm{R}_{\mathrm{F}} \\
\frac{\mathrm{dFR}}{\mathrm{dt}}=\mathrm{k}_{\text {on }} \cdot(\mathrm{F}-1) \cdot \mathrm{R}_{\mathrm{F}}-\mathrm{k}_{\mathrm{T}} \cdot \mathrm{FR}
\end{gathered}
$$

In the absence of exogenous cortisol $\left(\mathrm{w}_{\mathrm{Fex}}=0\right)$, the active steroid signal, FR(N), is normalized so that numerically it ranges between $(0,1)$. Thus, any increase in the concentration of the active signal $F R(N)$ will be relative to the trajectory that is elicited upon the systemic inflammatory manifestations of human endotoxemia. Regarding the immunosuppressive effects of glucocorticoids, antecedent periods of exogenouslyinduced hypercortisolemia attenuate circulating levels of pro-inflammatory cytokines through an increase in plasma IL-10 concentrations during human endotoxemia (van der Poll, Barber et al. 1996). For purposes of this model, it is assumed that cortisol modulates the host response to endotoxin primarily via potentiation of IL-10 signaling (A). Such anti-inflammatory influence is quantified through the linear stimulatory function, $\mathrm{H}_{\mathrm{A}, \mathrm{FRN}}$, which is discussed below in equation (6.12). 
Along the same lines, catecholamines such as epinephrine, (EPI), also modulate a range of immune functions (Padgett and Glaser 2003). Such hormones are secreted by the sympathetic nervous system pathway (SNS) and act via adrenergic receptors on immune cells (van der Poll 2000). In addition, there is evidence indicating that the proinflammatory response $(\mathrm{P})$ stimulates central components of the stress system through the afferent vagus nerve (Elenkov, Wilder et al. 2000). We will therefore assume that the pro-inflammatory response $(\mathrm{P})$ acts as the peripheral immune signal that not only stimulates the secretion of cortisol but also the secretion of epinephrine. The afferent transit mechanism that describes the propagation of the local pro-inflammatory signal (P) to the sympathetic nervous system (SNS) is shown in equations $(6.5)-(6.7)$.

$$
\begin{gathered}
\frac{\mathrm{dEPI}}{\mathrm{dt}}=\mathrm{w}_{\mathrm{EPI}, \mathrm{ex}} \cdot \mathrm{R}_{\mathrm{in}, \mathrm{EPI}}+\mathrm{K}_{\mathrm{in}, \mathrm{EPI}} \cdot\left(1+\mathrm{H}_{\mathrm{EPI}, \mathrm{P}}\right)-\mathrm{K}_{\text {out }, \mathrm{EPI}} \cdot \mathrm{EPI} \\
\mathrm{H}_{\mathrm{EPI}, \mathrm{P}}=\mathrm{k}_{\mathrm{EPI}, \mathrm{P}} \cdot \mathrm{P} \\
\mathrm{W}_{\mathrm{EPI}_{\mathrm{ex}}}=\left\{\begin{array}{l}
1, \text { exogenous hormone } \\
0, \text { otherwise }
\end{array}\right.
\end{gathered}
$$

The dynamics of epinephrine, Eq. (6.5), are described in the same manner as in Eq. (6.1) where total epinephrine concentration is defined as the joint effect between endogenous and exogenous hormone. Upon the systemic inflammatory manifestations of human endotoxemia, the rate of change of total epinephrine concentration (EPI), Eq. (6.5), is described by a zero order production rate $\left(\mathrm{K}_{\mathrm{in}, \mathrm{EPI}}\right)$ stimulated by the pro-inflammatory response $(\mathrm{P})$ through the linear function $\left(\mathrm{H}_{\mathrm{EPI}, \mathrm{P}}\right)$, Eq. (6.6) and a first order degradation rate $\left(\mathrm{K}_{\mathrm{out}, \mathrm{EPI}}\right)$. Further, in the case of exogenous epinephrine, the stimulatory effect of such perturbation is simulated via the parameter $\left(\mathrm{R}_{\mathrm{in}, \mathrm{EPI}}\right)$ which becomes activated in the presence of exogenous epinephrine controlled by the binary variable ( $\mathrm{W}_{\mathrm{EPIex}}$ ). 
Though pro-inflammatory cytokines stimulate the secretion of epinephrine, the latter attenuates the pro-inflammatory manifestations of human endotoxemia as supported by reduced TNF levels (van der Poll, Coyle et al. 1996). The anti-inflammatory influence of epinephrine is shown to be mediated by $\beta$ adrenergic stimulation resulting in an increase in cAMP intracellular levels followed by potentiation in the production rate of IL-10 signaling (A) (van der Poll, Coyle et al. 1996; van der Poll 2000). In order to mathematically describe such post-receptor effect, a precursor-dependent indirect response model (Mager, Wyska et al. 2003) is proposed where the precursor reflects the signaling receptor of epinephrine $\left(\mathrm{R}_{\mathrm{EPI}}\right)$ as shown in equations $(6.8)-(6.11)$.

$$
\begin{gathered}
\frac{\mathrm{dR}_{\mathrm{EPI}}}{\mathrm{dt}}=\mathrm{k}_{\mathrm{R}_{\mathrm{EPI}}}^{0}-\left[\mathrm{k}_{1, \mathrm{R}_{\mathrm{EPI}}} \cdot\left(1+\mathrm{H}_{\mathrm{R}_{\mathrm{EP}}, \mathrm{EPI}}\right)+\mathrm{k}_{2, \mathrm{R}_{\mathrm{EPI}}}\right] \cdot \mathrm{R}_{\mathrm{EPI}} \\
\frac{\mathrm{dEPIR}}{\mathrm{dt}}=\mathrm{k}_{1, \mathrm{R}_{\mathrm{EPI}}} \cdot\left(1+\mathrm{H}_{\mathrm{R}_{\mathrm{EPI}}, \mathrm{EPI}}\right) \cdot \mathrm{R}_{\mathrm{EPI}}-\mathrm{k}_{3, \mathrm{EPIR}} \cdot \mathrm{EPIR}-\mathrm{k}_{3, \mathrm{EPIR}} \\
\frac{\mathrm{dcAMP}}{\mathrm{dt}}=\frac{1}{\tau} \cdot\left((1+\mathrm{EPIR})^{\mathrm{n}}-\mathrm{cAMP}\right) \\
\mathrm{H}_{\mathrm{R}_{\mathrm{EPI}}, \mathrm{EPI}}=\mathrm{k}_{\mathrm{R}_{\mathrm{EPI}}} \cdot \mathrm{EPI}
\end{gathered}
$$

The dynamic changes of epinephrine receptor $\left(\mathrm{R}_{\mathrm{EPI}}\right)$ depend on an apparent zero-order |production rate $\mathrm{k}_{\text {REPI }}^{0}$ and $\mathrm{k}_{1, \mathrm{REPI}}, \mathrm{k}_{2, \mathrm{REPI}}$ represent first-order rate constants for the loss of the receptor, Eq. (6.8). Since the response is triggered as a result of the formation of an activating complex associated with the binding of EPI to its receptor, EPIR represents the formed signaling complex which decays with a first-order rate $\mathrm{k}_{3, \mathrm{EPI}}$, (6.9). Further, the stimulatory post-adrenergic effect of sympathetic activity in favoring the production of cAMP signaling is described by the principles of a signal transduction model as outlined in (Mager and Jusko 2001; Sun and Jusko 1998). In particular, the production and loss of 
the cAMP signaling depends on first-order rate constants which are equivalent to the reciprocal of the transit times $(\tau)$ consistent with the transit compartment model while $\mathrm{n}$ is the shaping (scaling) factor, Eq. (6.10) (Mager, Wyska et al. 2003). Such a scaling factor is used to amplify the signal transduction cascade associated with the post-adrenergic effect of epinephrine on the host. Regarding the immunosuppressive effect of epinephrine, a cAMP-dependent potentiation in IL-10 signaling (A) is quantified in Eq. (6.12) through the linear stimulatory function $\mathrm{H}_{\mathrm{A}, \mathrm{cAMP}}$. In addition to this, we previously mentioned that cortisol also increases IL-10 levels. Thus, such steroid-dependent immunomodulatory effect is quantified via the stimulatory function $\mathrm{H}_{\mathrm{A}, \mathrm{FRN}}$.

$$
\begin{aligned}
& \frac{\mathrm{dA}}{\mathrm{dt}}=\mathrm{K}_{\mathrm{in}, \mathrm{A}} \cdot\left(1+\mathrm{H}_{\mathrm{A}, \mathrm{cAMP}}\right) \cdot\left(1+\mathrm{H}_{\mathrm{A}, \mathrm{FRN}}\right) \cdot\left(1+\mathrm{H}_{\mathrm{A}, \mathrm{E}}\right)-\mathrm{K}_{\mathrm{out}, \mathrm{A}} \cdot \mathrm{A} \\
& \mathrm{H}_{\mathrm{A}, \mathrm{cAMP}}=\mathrm{k}_{\mathrm{A}, \mathrm{cAMP}} \cdot \mathrm{cAMP} \\
& \mathrm{H}_{\mathrm{A}, \mathrm{E}}=\mathrm{k}_{\mathrm{A}, \mathrm{E}} \cdot \mathrm{E} \\
& \mathrm{H}_{\mathrm{A}, \mathrm{FRN}}=\mathrm{k}_{\mathrm{A}, \mathrm{FRN}} \cdot \mathrm{FR}(\mathrm{N})
\end{aligned}
$$

In addition to the neuro-endocrine response evoked by endotoxin is the evolving concept of autonomic dysfunction as assessed by HRV indices. Recent studies imply that disordered neuro-endocrine functions are also associated with diminished HRV in stressed patients (Lowry and Calvano 2008). In order to quantify systemic abnormalities, clinical measurements of HRV will be further incorporated.

\subsection{A model for the assessment of reduced heart rate variability in human endotoxemia}

Clinical data associated with HRV measurements (Lowry 2005) establish that the host response to endotoxin causes a depression in both cardiac-vagal tone and in overall HRV 
and are consistent with prior studies (Godin, Fleisher et al. 1996; Rassias, Holzberger et al. 2005) indicating diminished physiologic variability as a generalized response to human endotoxemia. Several studies have implied the use of HRV as a readily available vital sign (Huikuri, Makikallio et al. 1999) in the assessment of critically ill patients with the hope of earlier intervention for those patients deemed at higher risk (Morris, Norris et al. 2006; Morris, Norris et al. 2007; Norris, Ozdas et al. 2006; Winchell and Hoyt 1997). Thus, the prognostic significance of HRV has made it a critical enabler to detect either physiologic deterioration or response to therapy (Winchell and Hoyt 1996).

In order to quantify the effect of acute endotoxin injury on heart rate variability a critical question that arises involves the relationship between pro-inflammatory markers and autonomic dysfunction. There is considerable human evidence indicating that systemic low-grade (pro)inflammatory activity is associated with reduced heart rate variability (Aronson, Mittleman et al. 2001; Malave, Taylor et al. 2003; Marsland, Gianaros et al. 2007). Assuming a linear relationship between pro-inflammation and HRV would imply that any modulations in the peripheral immune response will subsequently drive changes in hemodynamic parameters. However, it is important to realize that it cannot be taken for granted that factors modifying the magnitude of the immune response should affect all circulatory parameters equally (Bendixen, Osgood et al. 1964).

Such non-linearity in the sinus node transduction processes may arise from sensitivity of pacemaker discharge to the timing of pulsatile neural activity and from functional inhomogeneity within the sinus node tissue (Brown and Eccles 1934). In addition to this, the concept of non-linearity in cardiovascular variability has been stressed in the 
hemodynamic parameters of endotoxin-induced systemic inflammation under conditions of prior endocrine stress hormone infusion (Alvarez, Katsamanis Karavidas et al. 2007; Jan, Coyle et al. 2009). One of the interesting observations was that the antiinflammatory influence of endocrine hormones including cortisol and epinephrine does not extend to changes in heart rate variability induced by a relatively low dose of the inflammatory stimulus (LPS). Thus, reduction in endotoxin-induced pro-inflammation does not influence autonomic dysfunction (HRV), at least in a context of self-limited systemic inflammatory disease that resolves within 12-24 hours.

In order to quantify such non-linear interactions, the effect of peripheral proinflammation upon HRV response to endotoxin will be assumed that it can be mathematically approximated by employing appropriate sigmoid activation functions as outlined in (Berntson, Cacioppo et al. 1991). Although the overall heart rate variability is assessed, for instance, by evaluating the standard deviation of normal to normal interbeat intervals, the physiological background for such variation involves the activation of signal transduction mechanisms in the sinus node of the heart associated with the modulation of neuromediator concentrations (Zaza and Lombardi 2001). Thus, we introduce the signal $\mathrm{S}_{\mathrm{f}}$ as a surrogate for the up-regulation of such transduction processes in the heart and the relevant dynamics are described in equations (6.13) - (6.16). 


$$
\begin{gathered}
\frac{\mathrm{df}_{\mathrm{P}}}{\mathrm{dt}}=\left(1+\tanh (\mathrm{P}-\mathrm{w})-\mathrm{f}_{\mathrm{P}}\right) \cdot \mathrm{H}_{\mathrm{P}} \\
\frac{\mathrm{dS}_{\mathrm{f}}}{\mathrm{dt}}=\frac{1}{\tau_{\mathrm{S}}} \cdot\left(\mathrm{H}_{\mathrm{P}} \cdot \mathrm{f}_{\mathrm{P}}^{\mathrm{n}_{\mathrm{S}}}-\mathrm{S}_{\mathrm{f}}\right) \\
\frac{\mathrm{dHRV}}{\mathrm{dt}}=\mathrm{K}_{\mathrm{in}, \mathrm{HRV}}-\mathrm{K}_{\text {out }, \mathrm{HRV}} \cdot\left(1+\mathrm{k}_{\mathrm{HRV}, \mathrm{S}} \cdot \mathrm{S}_{\mathrm{f}}\right) \cdot \mathrm{HRV} \\
\mathrm{H}_{\mathrm{P}}=\tanh \left(\mathrm{P}^{\phi}-1\right)^{\phi}
\end{gathered}
$$

The possible non-linear modulatory effect of pro-inflammation (P) upon HRV is described by the dynamics of $f_{P}$, Eq. (6.13), where the switch-like behavior is determined by the sigmoid function $(\tanh (\mathrm{P}-\mathrm{w}))$ and $\mathrm{w}$ is a parameter greater than the proinflammatory response (P) elicited upon endotoxin-induced inflammation. This nonlinear gain modulatory function should be active under conditions of an inflammatory response and inactive when the system lies in its homeostasis. We therefore model such event based on the function, $H_{P}$, Eq. (6.16), where $\varphi$ is an M-big number and $H_{P}$ takes values zero, when pro-inflammation (P) lies in its baseline (homeostasis) and one otherwise. The underlying rationale for this function is predicated upon a neurocomputational model (Gutkin, Dehaene et al. 2006) which aims at simulating the firing rate of neuronal activity. In our model, $\mathrm{f}_{\mathrm{P}}$ could therefore reflect the activation of efferent nerve activity on the heart eventually leading to the up-regulation of intracellular mediators, $\left(\mathrm{S}_{\mathrm{f}}\right)$. The loss and production of such mediators, Eq. (6.14) is thereby described by the principles of time-dependent transduction systems (Mager, Wyska et al. 2003) depending on first-order rate constants which are equivalent to the reciprocal of the transit times $\left(\tau_{\mathrm{S}}\right)$ and $\mathrm{n}_{\mathrm{S}}$ is the shaping (scaling) factor, Eq. (6.14). The dynamics of HRV, 
(6.15) are described by a zero order production rate $\left(\mathrm{K}_{\mathrm{in}, \mathrm{HRV}}\right)$ and a first order degradation rate $\left(\mathrm{K}_{\mathrm{out}, \mathrm{HRV}}\right)$ which is stimulated by the effector biological signal $\left(\mathrm{S}_{\mathrm{f}}\right)$. Taken together, the integrated module that describes critical aspects of the neuro-endocrine immune system interactions is presented in equations $(6.17)-(6.19)$.

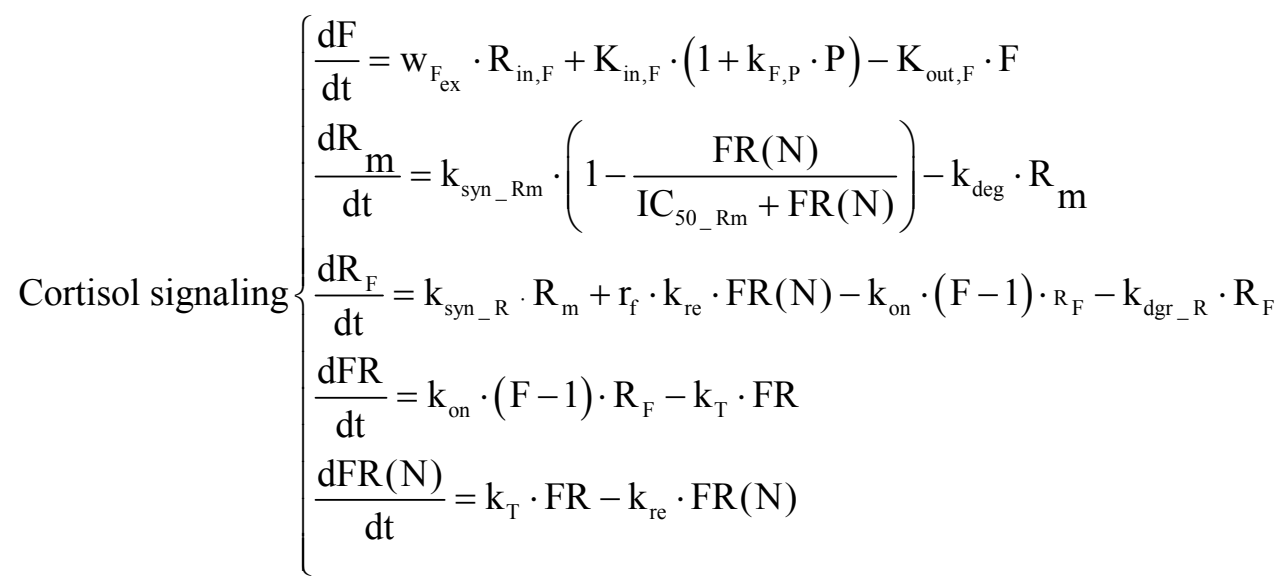

Epinephrine signaling $\left\{\begin{array}{l}\frac{\mathrm{dEPI}}{\mathrm{dt}}=\mathrm{w}_{\mathrm{EP}, \mathrm{ex}} \cdot \mathrm{R}_{\mathrm{in}, \mathrm{EPI}}+\mathrm{K}_{\mathrm{in}, \mathrm{EPI}} \cdot\left(1+\mathrm{k}_{\mathrm{EPI}, \mathrm{P}} \cdot \mathrm{P}\right)-\mathrm{K}_{\mathrm{out}, \mathrm{EPI}} \cdot \mathrm{EPI} \\ \frac{\mathrm{dR}}{\mathrm{EPI}}=\mathrm{k}_{\mathrm{R}_{\mathrm{EPI}}}^{0}-\left[\mathrm{k}_{1, \mathrm{R}_{\mathrm{EPI}}} \cdot\left(1+\mathrm{k}_{\mathrm{R}_{\mathrm{EPI}}, \mathrm{EPI}} \cdot \mathrm{EPI}\right)+\mathrm{k}_{2, \mathrm{R}_{\mathrm{EPI}}}\right] \cdot \mathrm{R}_{\mathrm{EPI}} \\ \frac{\mathrm{dEPIR}}{\mathrm{dt}}=\mathrm{k}_{1, \mathrm{R}_{\mathrm{EPI}}} \cdot\left(1+\mathrm{k}_{\mathrm{R}_{\mathrm{EPI}}, \mathrm{EPI}} \cdot \mathrm{EPI}\right) \cdot \mathrm{R}_{\mathrm{EPI}}-\mathrm{k}_{3, \mathrm{EPIR}} \cdot \mathrm{EPIR}-\mathrm{k}_{3, \mathrm{EPIR}} \\ \frac{\mathrm{dcAMP}}{\mathrm{dt}}=\frac{1}{\tau} \cdot\left((1+\mathrm{EPIR})^{\mathrm{n}}-\mathrm{cAMP}\right)\end{array}\right.$

Systemic (HRV) dynamics $\left\{\begin{array}{l}\frac{\mathrm{df}_{\mathrm{P}}}{\mathrm{dt}}=\left(1+\tanh (\mathrm{P}-\mathrm{w})-\mathrm{f}_{\mathrm{P}}\right) \cdot \mathrm{H}_{\mathrm{P}} \\ \frac{\mathrm{d} \mathrm{S}_{\mathrm{f}}}{\mathrm{dt}}=\frac{1}{\tau_{\mathrm{S}}} \cdot\left(\mathrm{H}_{\mathrm{P}} \cdot \mathrm{f}_{\mathrm{P}}^{\mathrm{n}}-\mathrm{S}_{\mathrm{f}}\right) \\ \frac{\mathrm{dHRV}}{\mathrm{dt}}=\mathrm{K}_{\mathrm{in}, \mathrm{HRV}}-\mathrm{K}_{\mathrm{out}, \mathrm{HRV}} \cdot\left(1+\mathrm{k}_{\mathrm{HRV}, \mathrm{S}} \cdot \mathrm{S}_{\mathrm{f}}\right) \cdot \mathrm{HRV}\end{array}\right.$

$\mathrm{H}_{\mathrm{i}, \mathrm{j}}=\mathrm{k}_{\mathrm{i}, \mathrm{j}} \cdot \mathrm{J}, \mathrm{w}_{\mathrm{i}, \mathrm{ex}}=\left\{\begin{array}{l}1, \text { exogenous hormone } \\ 0, \text { elsewhere }\end{array}, \mathrm{i}=\left\{\begin{array}{l}\mathrm{F}, \text { cortisol } \\ \text { EPI, epinephrine }\end{array}, \mathrm{H}_{\mathrm{P}}=\tanh \left(\mathrm{P}^{\phi}-1\right)^{\phi}\right.\right.$ 
While the model of equations (5.31) - (5.36) aims to describe the key determinants of the cellular response to endotoxin, the elements that constitute the module in equations (6.17) - (6.19) intend to connect the (cellular) inflammatory response with neural based pathways (HPA, SNS) while systemic disruptions are assessed by physiologic variables such as HRV.

Data used in this model linking extracellular signals with hormonal and physiological components include plasma concentration of counter-regulatory hormones such as cortisol and epinephrine (Alvarez, Katsamanis Karavidas et al. 2007; Barber, Coyle et al. 1993). Specifically, cortisol levels were measured at $0, .5,1,1.5,2,3,4,6$, and $24 \mathrm{~h}$ in relation to endotoxin administration (Alvarez, Katsamanis Karavidas et al. 2007) while the study period for epinephrine levels was $0,2,4$ and $6 \mathrm{~h}$ after endotoxin administration (Barber, Coyle et al. 1993). Further, human volunteers were injected with the same amount of LPS while vital signs, including heart rate variability indices were recorded (Alvarez, Katsamanis Karavidas et al. 2007). There are two basic approaches to quantifying heart rate variability, namely time-domain methods and frequency-domain (spectral) analyses (Berntson, Bigger et al. 1997).

In time-domain analysis, the heart rate at any time is determined from the time interval between successive respiration peaks in the QRS complexes (resulting from sinus node depolarization) of ECG (RR intervals). From the distribution of RR intervals, statistical measures of variance such as standard deviation of the time interval between consecutive respiration peaks and the root-mean square of the difference between adjacent RR intervals are determined. The root mean square of the successive beat differences is the recommended estimate of short-term variability while the standard 
deviation of normal interbeat intervals (SDNN) is for assessing longer-term variability (Berntson, Bigger et al. 1997). In addition to this, other time-domain measures that quantify the physiologic complexity between organ systems include multiscale entropy (MSE) (Costa, Goldberger et al. 2002). However, SDNN and MSE both quantify the complexity of interactions between organ systems and generate equivalent results when predicting mortality in intensive care unit (Norris, Stein et al. 2008).

Analysis of HRV in the frequency domain requires more complex algorithms but provides additional information. Frequency-domain measurements using Fourier analysis calculate the power of selected frequencies within a given frequency range (i.e. parasympathetic frequency ranges) (Winchell and Hoyt 1996). Thus, spectral methods produce a decomposition of total variation of a data series into its frequency components, which reflect operation of a particular modulatory reflex. For instance, parasympathetic (vagal) function is, oftentimes, assessed using the high frequency HRV while low frequency variability is a measure associated with both sympathetic and parasympathetic activation (Huikuri, Makikallio et al. 1999).

Although a wide variety of estimates of heart rate variability have been employed including both global descriptive statistics and spectral methods in this study, the timedomain measure SDNN will be used to assess overall heart rate variability which will serve as a surrogate for systemic abnormalities. During the analysis of heart rate variability, parameters and inter-beat intervals were collected using ECG data at a rate of 256 samples/s where each QRS complex (which corresponds to the depolarization of the ventricles) was detected and the "normal-to-normal" (NN) intervals were tabulated. HRV measurements and plasma cortisol concentrations are employed from (Alvarez, 
Katsamanis Karavidas et al. 2007) while epinephrine concentrations are also assessed under the systemic inflammatory manifestations of human endotoxemia (Barber, Coyle et al. 1993). The data have been appropriately de-identified and appropriate IRB approval and informed, written consent were obtained from the volunteers.

\subsubsection{Elements of the multiscale host response model of human inflammation}

We have previously demonstrated that the transcriptional dynamics of human leukocytes exposed to bacterial endotoxin can be decomposed into to three elementary comprehensive responses (Foteinou, Calvano et al. 2007). These elementary responses capture the functional dynamics and were shown to be related to pro-inflammatory $(\mathrm{P})$, anti-inflammatory (A) and energetic (E) transcriptional events associated with the overall host response. The response is triggered by the activation of the NF-kB signaling module as a result of the formation of an activating signal associated with the binding of LPS to appropriate receptors $(\mathrm{R})$. In order to introduce higher level biological information we

further incorporate critical aspects of the neuro-endocrine immune crosstalk. A schematic illustration of the network architecture that constitutes the multi-level host response model is presented in Figure 6.1. 


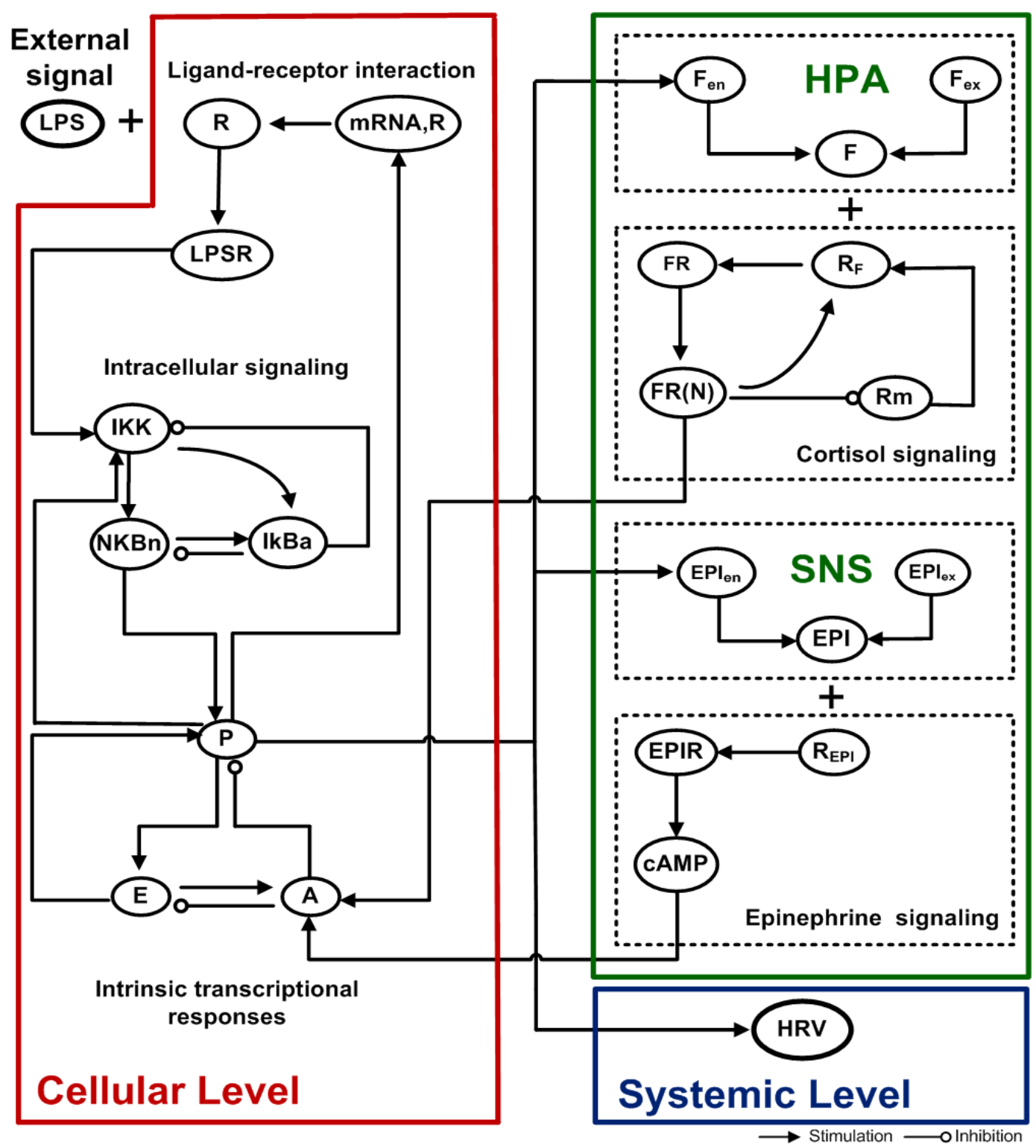

Figure 6.1: Basic topological interactions composing the multi-level model of endotoxin induced human inflammation. At the cellular level, interacting components involve the propagation of LPS signaling on the transcriptional response level $(\mathrm{P}, \mathrm{A}, \mathrm{E})$ through the activation of endotoxin signaling receptor $(\mathrm{R})$ and elementary signaling pathways (NF-kB signaling module). At the level of circulating hormones, essential modules are associated with the release of endocrine stress hormones from neuroendocrine axis (HPA, SNS) coupled with their anti-inflammatory influence on the host. The dynamics of cortisol and epinephrine signaling involve components interacting at the cellular level. At the systemic level, physiologic deterioration of the host is quantified by HRV. 
At the cellular level (Figure 6.1) interacting components are associated with elementary signaling pathways that propagate extracellular signals to the emergent transcriptional response level. Essential modules associated with the release of endocrine stress hormones coupled with their immunosuppressive effects are also considered. Such hormones are integral parts of the bi-directional communication pathway between peripheral inflammation (cellular level) and the neuro-endocrine axis (HPA, SNS) and interact with appropriate receptors potentiating the production rate of anti-inflammatory cytokines (A). Finally, clinical measurements, at the systemic level, of HRV are incorporated to assess systemic decomplexification manifested by deterioration in the physiologic status of the host.

\subsubsection{Estimation of relevant model parameters}

Standard parameter estimation techniques are applied in order to evaluate appropriate model parameters associated with the neuro-endocrine immune system interactions (Contreras and Ryan 2000). In particular, we estimate those model parameters that are involved in the dynamics of epinephrine (EPI), cortisol (F), anti-inflammation (A) and heart rate response $(\mathrm{HRV})$. The relative experimental data are normalized by taking the ratio of the measured response at each time point of the study period with respect to the control time point $(\mathrm{t}=0 \mathrm{hr})$. Further, parameter estimation is performed to estimate the parameter $\left(\mathrm{R}_{\mathrm{in}, \mathrm{F}}\right)$ under conditions of hydrocortisone infusion (exogenous cortisol) reproducing human plasma cortisol levels in subjects pre-exposed 6h prior to LPS and continued for another $6 \mathrm{~h}$ after the endotoxin injection. All the other parameters associated with the propagation of LPS signaling on the transcriptional response level are maintained to agree with those presented in (Foteinou, Calvano et al. 2009b) and are 
shown in Table 5.1 (see Appendix 5.4) while parameters relevant to the neuro-endocrine immune system interactions are estimated and presented in Table 6.1 (see Appendix 6.4). The performance of the multi-level human inflammation model is shown in Figure 6.2.
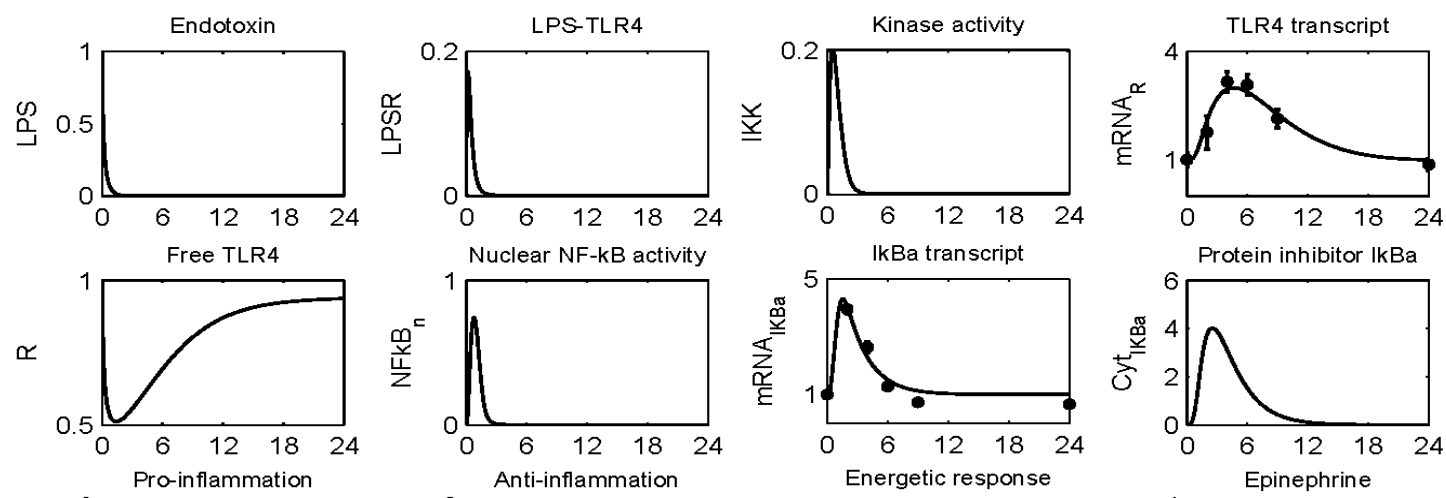

ㅁ
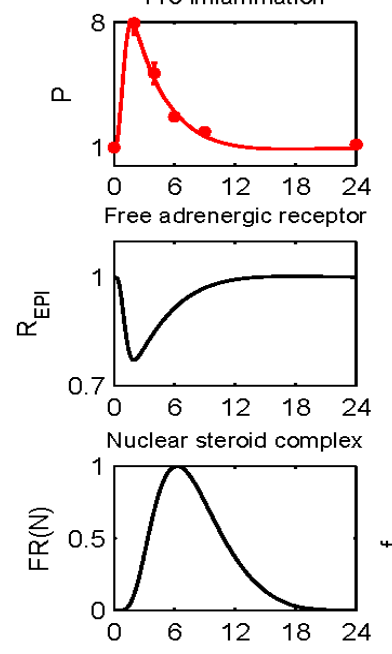

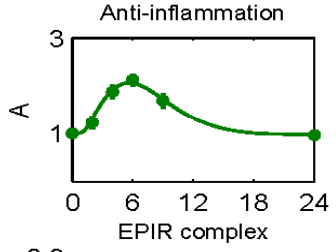

ш
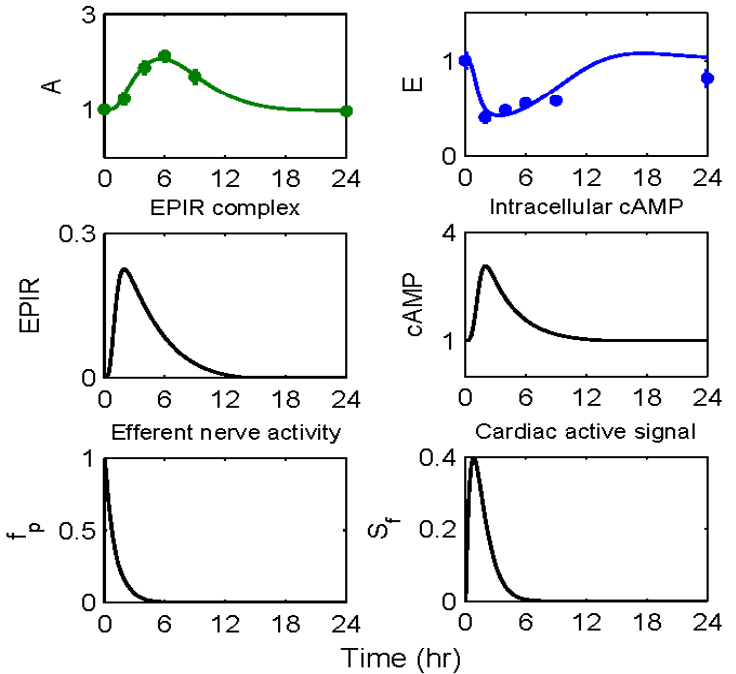
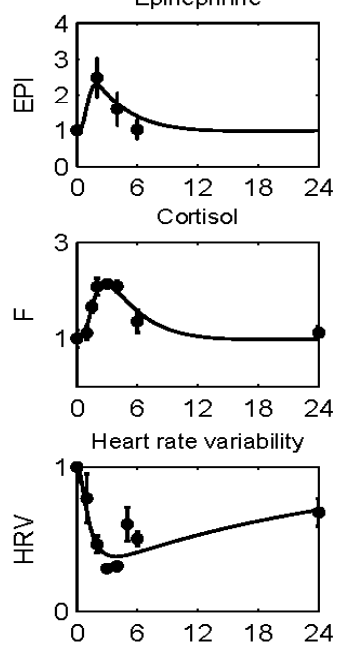

Figure 6.2: Basic topological interactions composing the multi-level model of endotoxin induced human inflammation. At the cellular level, interacting components involve the propagation of LPS signaling on the transcriptional response level (P, A, E) through the activation of endotoxin signaling receptor (R) and elementary signaling pathways (NF-kB signaling module). At the level of circulating hormones, essential modules are associated with the release of endocrine stress hormones from neuroendocrine axis (HPA, SNS) coupled with their anti-inflammatory influence on the host. The dynamics of cortisol and epinephrine signaling involve components interacting at the cellular level. At the systemic level, physiologic deterioration of the host is quantified by HRV. 
In our computational model the host restores homeostasis without any external perturbation. A self-limited inflammatory response to the endotoxin stimulus corresponds to resolved dynamic profiles for all the elements that constitute our model. In essence, a self-limited inflammatory response involves the successful elimination of the inflammatory stimulus within the first $2 \mathrm{hr}$ post-endotoxin administration while followed by a subsequent resolution within $24 \mathrm{hr}$. Though, the kinetic parameters associated with the epinephrine-receptor interactions are not calibrated, the dynamic profile of $\beta$ adrenergic receptor $\left(\mathrm{R}_{\mathrm{EPI}}\right)$ lies in qualitative agreement with the basis of receptor occupancy theory (Mager and Jusko 2001) in that the concentration of free adrenergic receptors decreases in the presence of the ligand (EPI). Regarding the gain modulatory effect of peripheral pro-inflammation in the heart $\left(f_{\mathrm{P}}\right)$, such an exponential decrease would biologically reflect the decay rate of cardiac neuronal activity (Lameris, de Zeeuw et al. 2002) which is eventually "translated" to the up-regulation of neuro-mediator concentrations $\left(\mathrm{S}_{\mathrm{f}}\right)$ in the heart. Further, the reconstruction of plasma cortisol levels under conditions of either prior cortisol infusion or LPS only are shown in Figure 6.3. While comparing the top and bottom panel of Figure 6.3, both plasma cortisol levels (F) and the steroid active signal, FR(N) are expected to be greater under conditions of exogenouslyinduced hypercortisolemia (bottom panel, $\mathrm{w}_{\mathrm{Fex}}=1$ ) relative to the baseline cortisol profiles (top panel, $\mathrm{w}_{\mathrm{Fex}}=0$ ). 

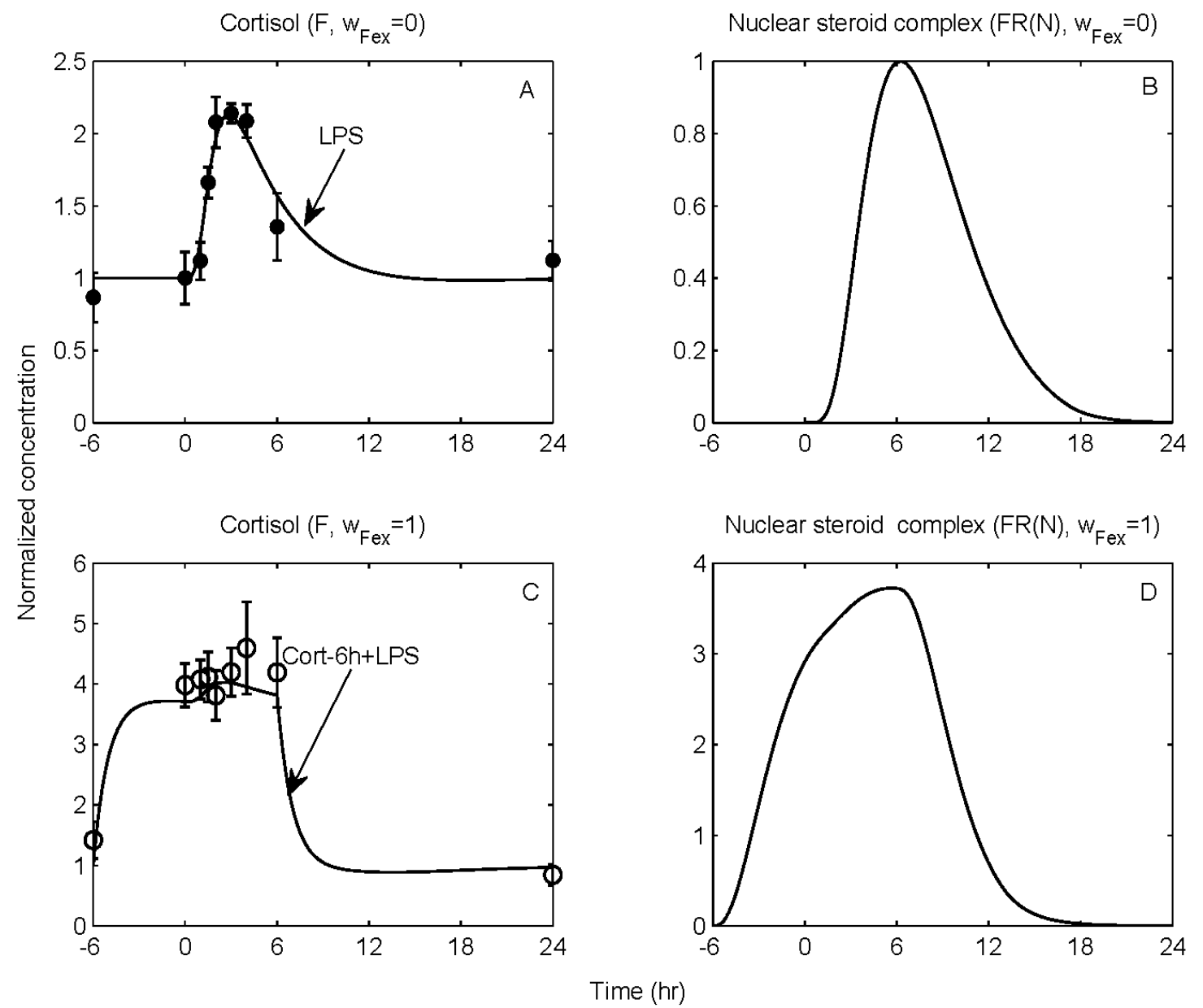

Figure 6.3: Estimation of relevant model parameters intending to reproduce available experimental data associated with transcriptional signatures (A) and plasma counter-regulatory hormones including epinephrine (EPI) and cortisol (F) as well as clinical data (HRV). Solid lines (-) correspond to model predictions under conditions of low-dose endotoxin while the symbols $(\bullet)$ refer to experimental data expressed as mean $\pm \mathrm{SEM}$. The initial condition of endotoxin $(\operatorname{LPS}(\mathrm{t}=0 \mathrm{~h})=1)$ refers to LPS concentration relative to $2-\mathrm{ng} / \mathrm{kg}$ body weight. 


\subsubsection{Qualitative assessment of the model}

Building a mathematical model that can predict relevant biological implications to the host response to endotoxin allows us to identify ways of both controlling and modulating such a complex phenomenon. In the following we will demonstrate the ability of our model to enable such "predictions" and provide further evidence of the appropriateness of the assumptions invoked in the development of the model. First, we explore the implications of increasing levels of initial insult (LPS) since this would probably constitute the most obvious disturbance. Then we explore possible reversibility in the dynamics of the host in response to an acute endocrine hormone stress infusion (cortisol, epinephrine excess). Finally, the implications of acute stress hormone infusion upon the systemic inflammatory manifestations of human endotoxemia will be evaluated. We opt therefore to validate the correctness of the proposed model by assessing the implications of anti-inflammatory treatment strategies that are active under conditions of either high or low infectious challenge.

\section{$\underline{\text { Implications of increased insult }}$}

An increase in the dose of the inflammatory stimulus can be responsible for an overwhelming inflammatory response. Such situation in which the initial levels of endotoxin are increased is simulated in Figure 6.4. This response can be equated with an exacerbated inflammatory state in the early phase of severe injury, which in our model is simulated as high concentration of the initial stimulus (LPS), i.e. four times greater than the nominal which corresponds to $8 \mathrm{ng} / \mathrm{kg}$, (Note: maximum dose of LPS is administered safely to humans is $4 \mathrm{ng} / \mathrm{kg}$ (Andreasen, Krabbe et al. 2008)), which deregulates the host defense intrinsic dynamics towards a cytokine "burst". 

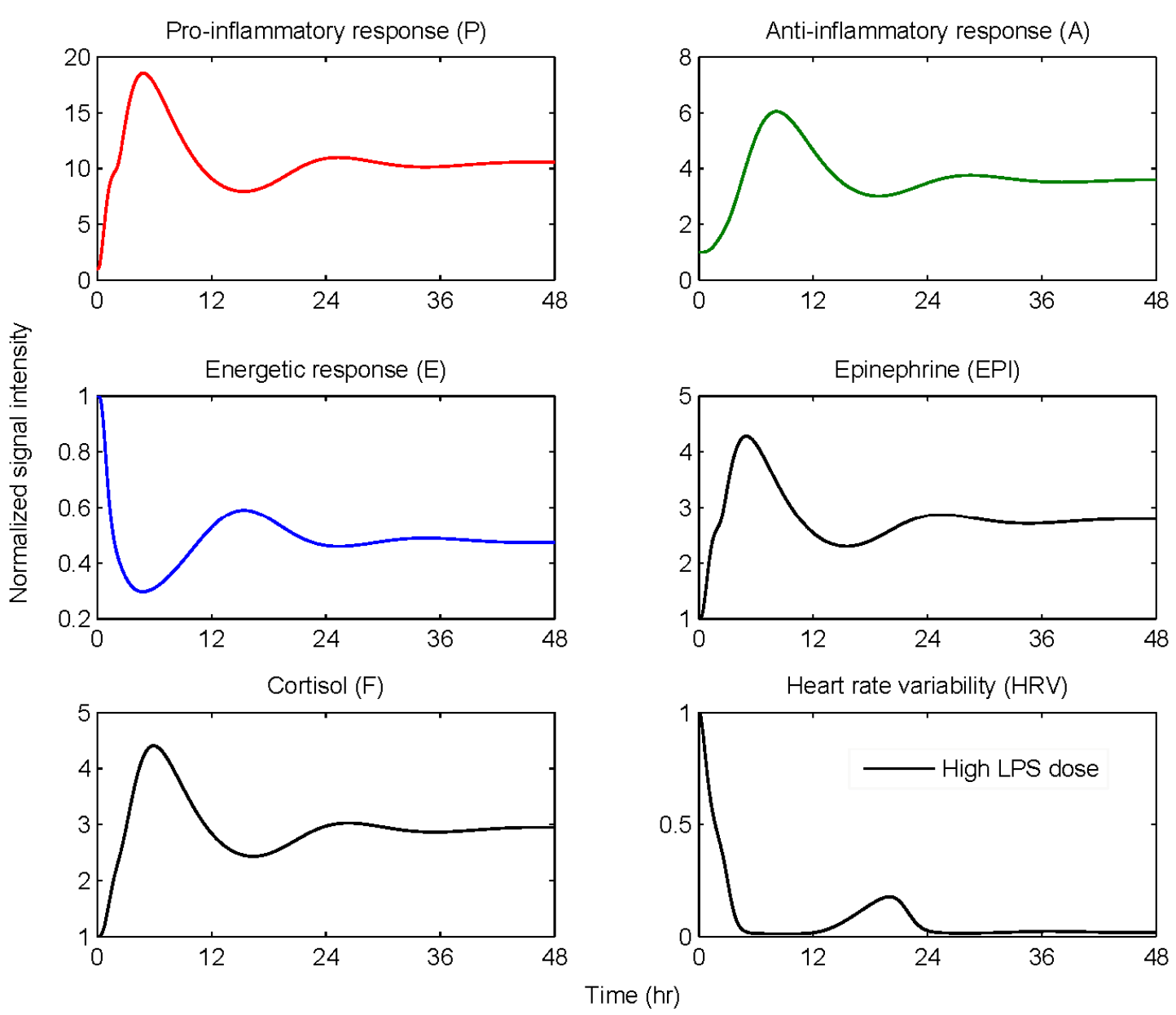

Figure 6.4: Simulation of an unresolved inflammatory response due to high endotoxin concentration (LPS $(\mathrm{t}=0 \mathrm{~h})=4)$. Such high concentration of LPS (four times greater than the nominal value) deregulates the NF-kB signaling module giving rise to an unconstrained immune response followed by abnormal hormonal responses that macroscopically are translated into diminished physiologic variability.

As shown in Figure 6.4 the cytokine "burst" is further accompanied by the uncontrolled secretion of endocrine hormones that are not adequate to balance (control) the overall immune response even after the circulating levels of LPS have been cleared. Phenotypically, such physiologic deterioration is expressed as diminished heart rate 
variability that does not return to baseline within $24 \mathrm{hr}$ (solid lines) as was seen in Figure 6.3 where a lower dose of LPS was simulated compared with experimental data. Such computational results implicate the role of the host rather than the inflammatory stimulus itself, which is eventually cleared from the system, accounting for the progression of an unconstrained inflammatory response. Qualitatively, this amplification of the host immune response (trajectories of inflammatory relevant components do not return to baseline-homeostasis) without present infection represents clinically stressed patients without documented infection (Reyes, Brimioulle et al. 1999).

\section{Evaluation of stress hormone infusion in modulating the inflammatory response}

Since we have demonstrated the ability of our model to simulate the trajectory of an unconstrained inflammatory response, the potential of the proposed model is also demonstrated through its capability to respond to systematic perturbations that modulate the dynamics in favor of a balanced immune response coupled with a restoration in autonomic balance. Considerable attention has been given to the effectiveness of pharmacological agents such as ligands of adrenergic receptors in influencing the production rate of both pro- and anti-inflammatory cytokines (Hasko, Elenkov et al. 1995; Ignatowski and Spengler 1995). In particular, significant modulations in the cytokine network was observed in human subjects exposed to epinephrine infusion (van der Poll, Coyle et al. 1996) underscoring the role of neuro-endocrine activity in dampening excessive pro-inflammatory effects. In particular, we opt to simulate the mode of an intervention strategy that mimics the activity of sympathetic nervous system pathway. Such intervention strategy results in potentiation of the total plasma 
concentration of epinephrine (EPI) which further increases the intracellular cAMP signaling (dashed lines) Figure 6.5.

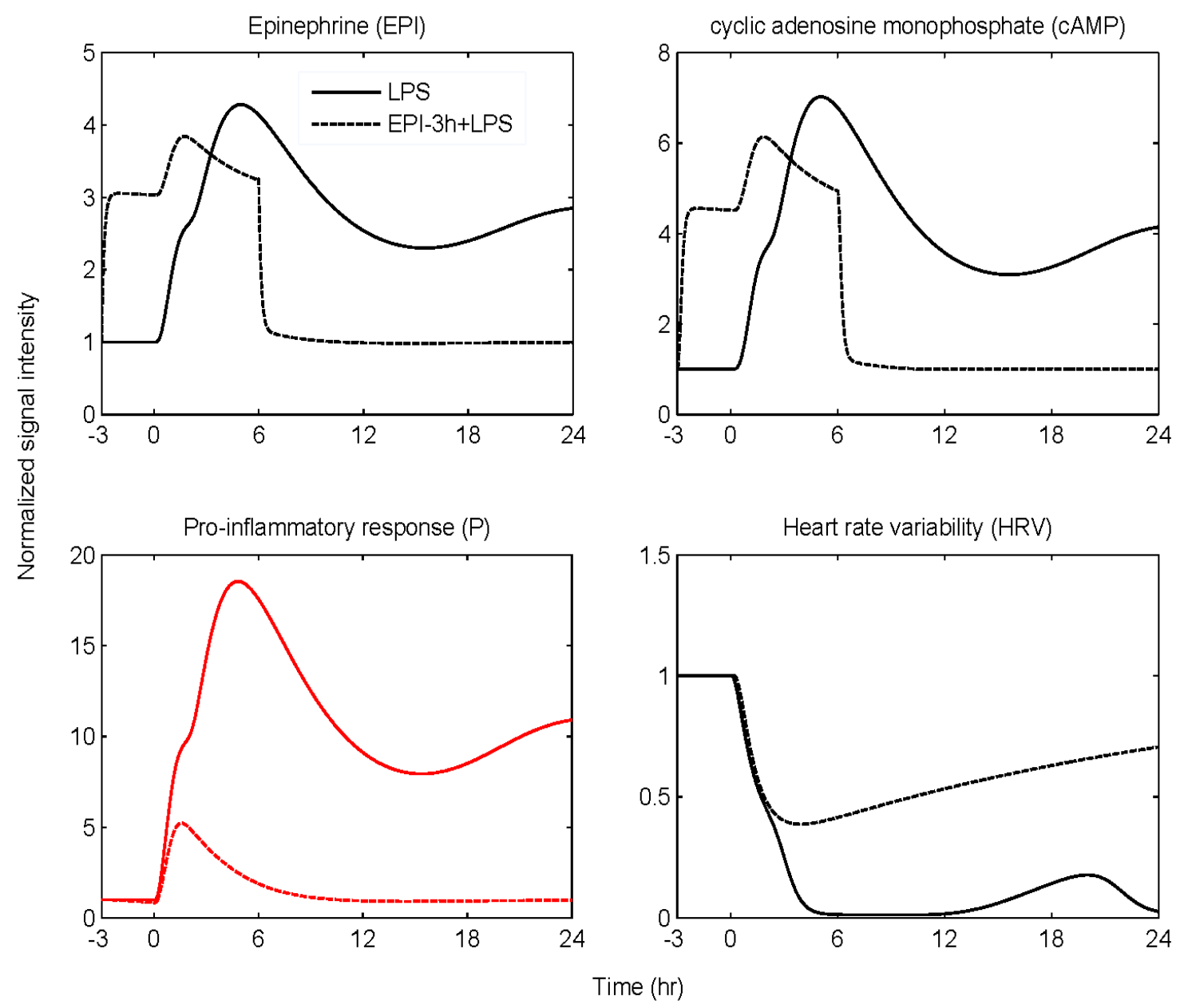

Figure 6.5: Simulating the effect of acute epinephrine infusion $\left(\mathrm{w}_{\mathrm{EPI}, \mathrm{ex}}=1\right)$, which is initiated $3 \mathrm{~h}$ prior to the main endotoxin challenge $(\operatorname{LPS}(t=0 h)=4)$ and continued for $6 \mathrm{hr}$ after LPS $\left(\mathrm{R}_{\mathrm{in}, \mathrm{EPI}}=15\right)$, under conditions of severe inflammation. Dashed and solid lines represent the progression of a balanced (due to system's pre-exposure into epinephrine infusion) and unconstrained inflammatory response (due to high inflammatory challenge, $\operatorname{LPS}(\mathrm{t}=0 \mathrm{~h})=4$ ) respectively. Acute pre-exposure of the host to epinephrine attenuates the aberrant pro-inflammatory response (P) induced by high LPS concentration which allows for recovery in HRV dynamics (restoration in autonomic balance). 
Based on the anti-inflammatory effect of acute EPI infusion via cAMP dependent mechanism, it is expected an increase in intracellular cAMP levels (Figure 6.5) to attenuate the pro-inflammatory response $(\mathrm{P})$ followed by a subsequent restoration in autonomic activity (HRV) which serves as a proxy indicator of improved survival (Stein and Kleiger 1999). Thus, the acute pre-exposure of the host to epinephrine attenuates the pro-inflammatory response $(\mathrm{P})$ which allows for recovery of HRV dynamics. Such improvement in autonomic activity underscores the role of epinephrine in improving cardiac index under severe conditions (i.e. low-output septic shock) as supported by Court et al (Court, Kumar et al. 2002).

Further the central nervous system controls inflammation through the activation of HPA axis by releasing cortisol. Prior studies evaluating human responses within the context of antecedent stress hormone excess have shown that glucocorticoid excess, as produced by hydrocortisone injection (Lin, Calvano et al. 1999) or 6-hr infusion before LPS challenge abrogates several features of human endotoxemia (Barber, Coyle et al. 1993). In an effort to assess the impact of such hypercortisolemia as a potential endogenous in vivo anti-inflammatory mechanism, hydrocortisone infusion is initiated 6hr before the administration of high LPS concentration, Figure 6.6. Note that in our model, high LPS dose which is simulated by simply varying the concentration of LPS at time zero, serves as one putative mode of dysregulation in the host defense intrinsic dynamics giving rise to unremitting inflammation. Prior mathematical models of inflammation also simulate severe inflammatory states by varying the initial conditions of the inflammatory insult (Chow, Clermont et al. 2005). 


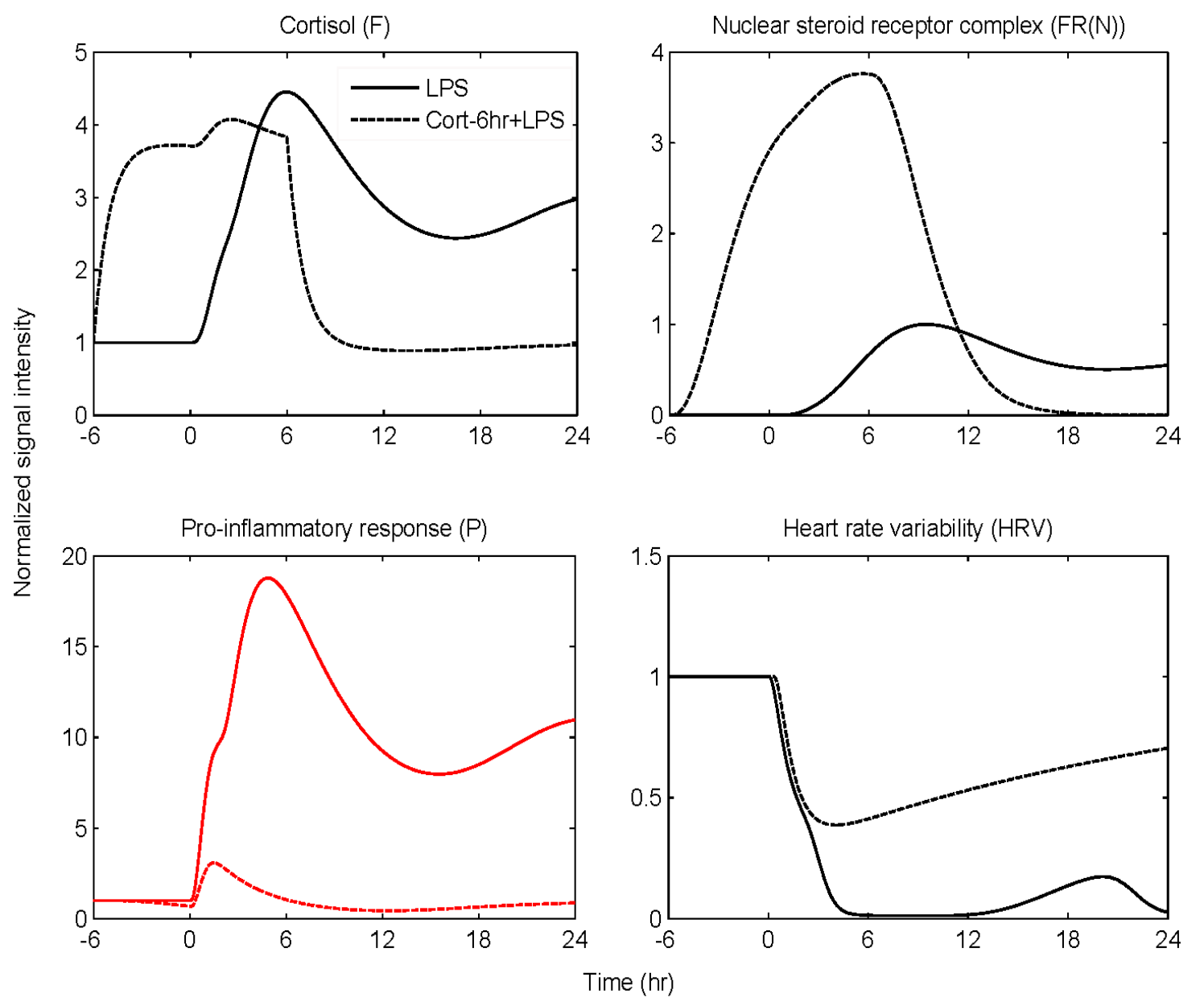

Figure 6.6: Explore the effect of low-dose steroid administration initiated $6 \mathrm{~h}$ prior to endotoxin challenge (dashed lines) while continued for another $6 \mathrm{~h}$ after LPS $\left(\mathrm{w}_{\mathrm{Fex}}=1\right)$ under conditions of high LPS concentration (solid lines). Solid lines simulate the progression of a systemic inflammatory response syndrome (due to high LPS concentration, LPS $(\mathrm{t}=0 \mathrm{~h})=4$ ) while dashed lines reflect the protective effect that can be exerted by hormonal(steroid) replacement therapy. The acute pre-exposure of the host to exogenous cortisol dampens the excessive pro-inflammatory effects induced by high LPS concentration which allows for restoration in autonomic balance (HRV).

The acute pre-exposure of the host into cortisol, as represented by dashed lines in Figure 6.6, attenuates pro-inflammatory responses that mitigate the subsequent amplified inflammatory response. Specifically, the initiation of such intervention strategy increases 
the total concentration of cortisol (F) which subsequently potentiates the active steroid signal $(\mathrm{FR}(\mathrm{N}))$. Such increase in total cortisol levels potentiates the anti-inflammatory arm of the system (A) immediately after the administration of LPS and thereby attenuates the pro-inflammatory response $(\mathrm{P})$. Thus, the initiation of such intervention strategy that indirectly attenuates the pro-inflammatory signaling $(\mathrm{P})$ via potentiation of the humoral anti-inflammatory signaling (A) suffices to reverse the inflammatory dynamics and eventually restore autonomic balance. Collectively, such in silico predictions as illustrated in Figure 6.5 and Figure 6.6 annotate the impact of dynamic antiinflammation on the host evoked by stress hormone background upon the systemic inflammatory manifestations of acute illnesses and suggest that the rates of this response may be well tuned to yield optimal outcomes.

Thus, a fundamental assumption of our model is the existence of two steady states that depending on the anti-inflammatory "reservoir" of the host can represent either "recovery/self-limited" or "uncontrolled/sustained HRV depression". Qualitatively, such equilibria might account for the transient clinical improvement (i.e. "survivors") noted to critically ill patients that respond to a treatment. For example, in the study (Morris, Norris et al. 2007) among injured patients there exists a subset of severely stressed patients whose clinical condition improved upon treatment with exogenous steroid. However, we would like to point out that a direct comparison between our model predictions and clinical observations is beyond the scope of the present study. Instead, the overall goal of this study is to develop an in silico model of human endotoxemia that would allow us to evaluate antecedent stresses upon the systemic inflammatory manifestations of acute injury. 
Although the immunosuppressive effects of corticosteroids upon the systemic inflammatory manifestations of human endotoxemia have been well described, the influence of this anti-inflammatory intervention on overall autonomic dysfunction is not well understood. Predicated upon this, the influence of steroid administration on a selflimited endotoxin-induced inflammatory response is simulated in Figure 6.7. Although measurements of the transcript abundance of cytokines are not available under such conditions, soluble inflammatory markers (i.e. TNF-a, IL-8, Il-10) were measured and were significantly modulated by prior hydrocortisone treatment. Specifically, antecedent cortisol infusion blunts the pro-inflammatory cytokine response to LPS administration while enhancing some anti-inflammatory responses as reflected by increased plasma IL10 concentrations (Alvarez, Katsamanis Karavidas et al. 2007; van der Poll, Barber et al. 1996). Predicated upon the hypothesis that cytokine protein expression correlates well with gene expression (Prabhakar, Conway et al. 2005), we seek to validate our model qualitatively by simulating an enhanced transcriptional anti-inflammatory response (A) followed by diminished pro-inflammation (P) under conditions of exogenously-induced hypercortisolemia as represented by dashed lines (Figure 6.7). In addition to such attenuation in the pro-inflammatory response, antecedent cortisol infusion also induces hormonal changes and particularly reduction in plasma epinephrine concentrations. Such decrease in endogenous epinephrine secretion under acute hypercortisolemia is simulated in Figure 6.7(panel E); thus validating the assumptions invoked in the development of the proposed integrated model. 

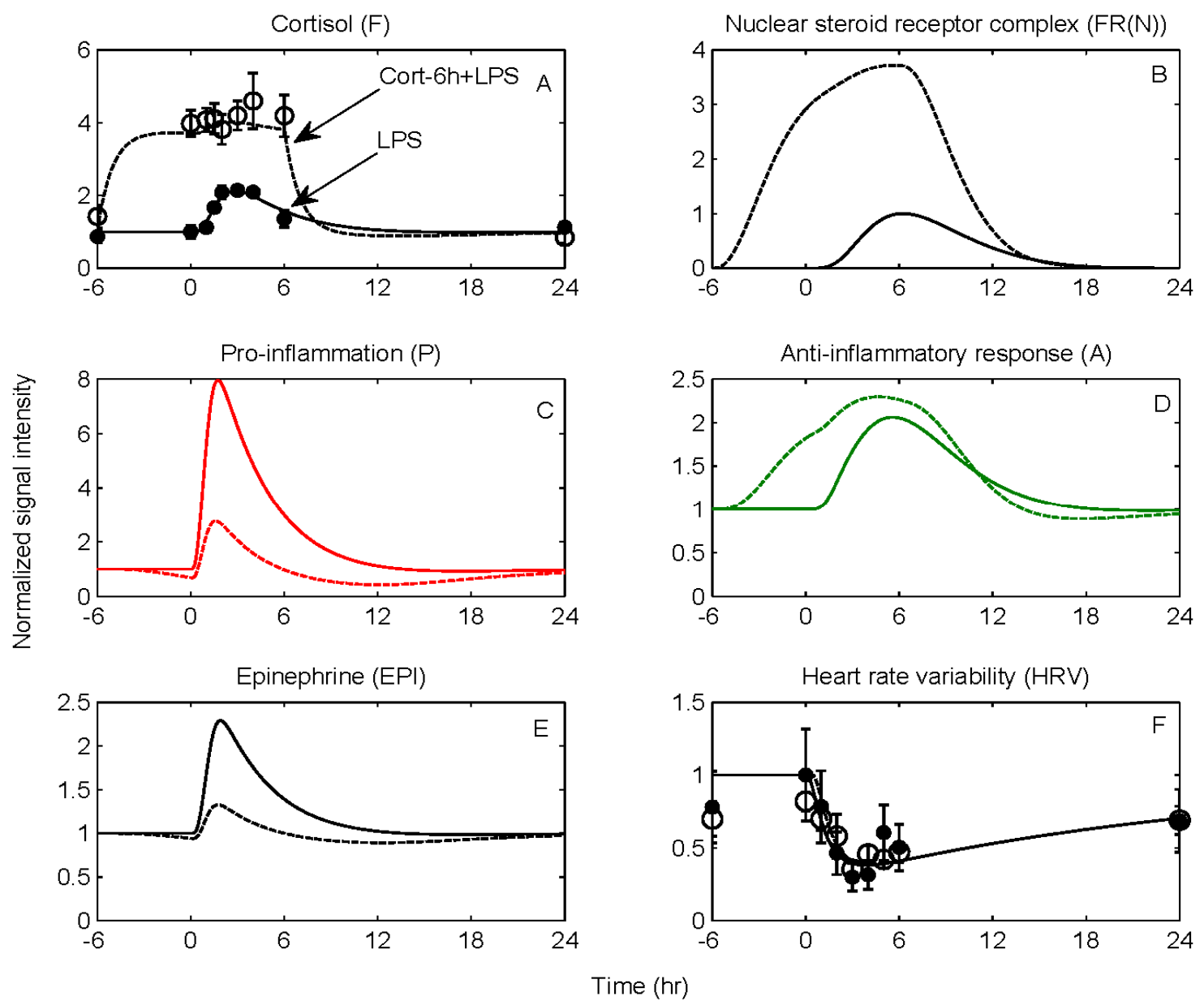

Figure 6.7: Explore the effect of exogenously-induced hypercortisolemia on autonomic dysfunction under the systemic inflammatory manifestations mediated by low-dose endotoxin. Solid lines simulate the progression of a self-limited endotoxin-induced inflammatory response while dashed lines reflect the antecedent period of exogenously-induced hypercortisolemia which is initiated $6 \mathrm{~h}$ prior to LPS administration and continued for $6 \mathrm{~h}$ after endotoxin $\left(\mathrm{w}_{\mathrm{Fex}}=1\right)$. Solid markers and open circles refer to experimental data (expressed as mean \pm SEM) under conditions of acute endotoxin injury and prior hydrocortisone infusion respectively which do not vary across the two experimental conditions (LPS, Cort$6 \mathrm{~h}+\mathrm{LPS})$. Such prior cortisol infusion modulates cytokine responses (P, A) and hormonal responses (EPI) but there is no change in overall system's adaptability as assessed by HRV (solid and dashed HRV lines overlap). 
Remarkably, although acute hypercortisolemia significantly attenuated endotoxininduced production of pro-inflammatory cytokines, such attenuation in a context of acute systemic inflammatory condition mediated by endotoxin administration (Alvarez, Katsamanis Karavidas et al. 2007) does not contribute to any alterations in HRV indices. From a computational standpoint, such effect is represented as superimposition of the solid and dashed lines in the HRV component as shown in Figure 6.7F.

In addition to the influence of low-dose steroid on endotoxin-induced inflammation, recent data document that prior EPI exposure may attenuate the pro-inflammatory response but such anti-inflammatory influence does not extend to changes in overall system's adaptability (HRV) (Jan, Coyle et al. 2009). Since increased catecholamine secretion accompanies modest infection and the propensity of a dose dependent effect of EPI in inhibiting LPS-induced pro-inflammatory response has been documented in (Van der Poll and Lowry 1997), we sought to simulate whether antecedent EPI infusion would modulate in a dose-dependent manner the cytokine responses to endotoxin, Figure 6.8. In particular, increasing doses of acute sterile stress condition modulate the innate immune system activation and particularly attenuate the pro-inflammatory response through potentiation of the anti-inflammatory effect of cAMP signaling. However, such attenuation in the progression of the pro-inflammatory response does not contribute to any changes in HRV response as experimentally observed (Jan, Coyle et al. 2009), and represented by the superimposition of predicted HRV dynamics in Figure 6.8 which is consistent with the aforementioned results with steroid administration before LPS. 

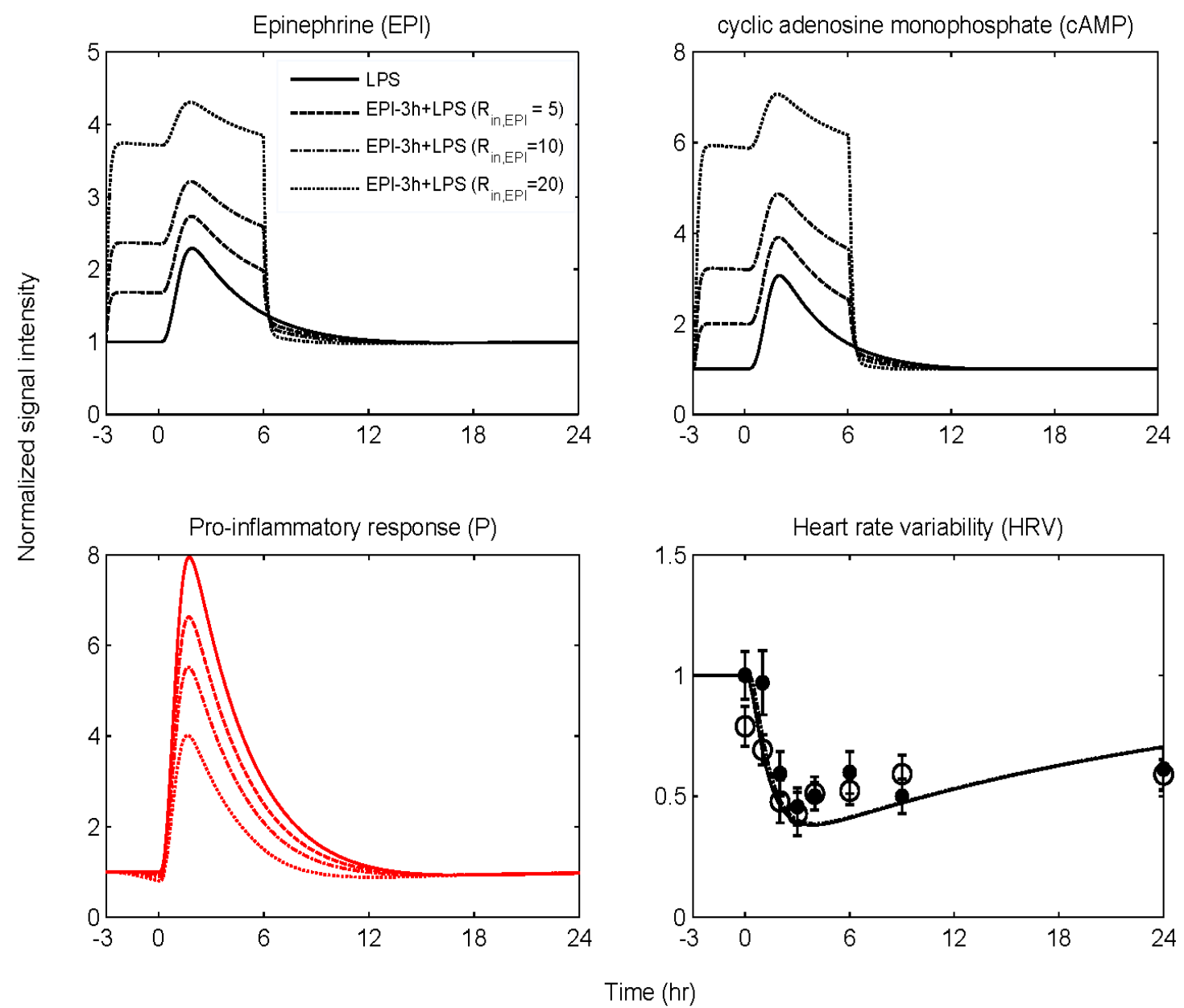

Figure 6.8: Dose-dependent modulation in the progression of the inflammatory reaction due to short-term epinephrine infusion $\left(\mathrm{w}_{\mathrm{EPI}, \mathrm{ex}}=1\right)$, initiated $3 \mathrm{~h}$ before LPS and continued for $6 \mathrm{~h}$ after LPS at increasing values of the parameter $R_{\text {in,EPI }}=5,10,20$. Such intervention potentiates, in a dose dependent manner (dashed and dotted lines), the secretion of epinephrine from SNS that through cAMP anti-inflammatory signaling can protect, in part, the host response attenuating the pro-inflammatory response (P). Such attenuation in the pro-inflammatory response relative to endotoxin administration (solid lines) does not extend to changes in autonomic balance (HRV) as represented by the superimposition of predicted HRV dynamics (solid and dashed lines overlap). Solid markers and open circles refer to relevant experimental data (expressed as mean \pm SEM) under conditions of low-dose endotoxin administration and prior epinephrine infusion respectively. These data have not been used as a training dataset but rather to validate the structure of the proposed model. Descriptive statistics in the original analysis (Jan, Coyle et al. 2009) 
show that there was no significant variation between these experimental measurements (solid markers vs. open circles) from $0 \mathrm{hr}$ until 24 hours after LPS exposure.

From a modeling standpoint, such responses are captured due to the possible nonlinear interaction between peripheral pro-inflammation and heart rate variability. In particular, a fundamental assumption of the proposed study is that any reduction in the pro-inflammatory response relative to the constrained response evoked by low-doses of endotoxin, will not affect the magnitude of HRV relative to the naïve (LPS) injection. Such assumption is primarily predicated upon evidence (Alvarez, Katsamanis Karavidas et al. 2007; Jan, Coyle et al. 2009) that indicate the existence of reduced differential proinflammatory responses within the context of antecedent stresses without altering endotoxin-induced HRV dynamics. On the other hand, under conditions of high inflammatory challenge, as illustrated in Figure 6.4, an unconstrained pro-inflammatory response will account for a persistent diminished physiologic variability (HRV) indicative of the severity of injury.

While the proposed model does not capture the sympathomimetic properties of epinephrine, we recognize that antecedent epinephrine infusion significantly reduced the parasympathetic tone and there was a relative decrease in HRV in the initial hours after EPI administration (Jan, Coyle et al. 2009). In the following section, we plan to describe dynamic changes in heart rate variability as a result of cardiac autonomic imbalance incorporating explicitly the interplay between efferent branches of the autonomic nervous system (sympathetic/parasympathetic outflow) that account for modulations in heart rate response. Such modeling extensions associated with the autonomic control of heart rate would allow us to simulate the vagolytic influence of EPI and therefore explore the 
possibility of developing more mechanistic-based and physiological relevant in silico disease progression models.

In summary, a multi-level human inflammation model is proposed that couples essential aspects of the complex bidirectional relationship between the neuro-endocrine axis and the immune response. We addressed how to construct the topology and the dynamics of the underlying network linking processes across the host from the cellular to the systemic level. Essential modules associated with the secretion of endocrine stress hormones (cortisol, epinephrine) and their counter-regulatory role are particularly taken into account while phenotypic expressions such as HRV are further incorporated to assess systemic decomplexification. The proposed work bridges the initiating signal and phenotypic expressions through semi-mechanistic based host response models that include transcriptional dynamics, signaling cascades and physiological (hormonal) components. Model parameters are appropriately evaluated so that to reproduce a selflimited inflammatory response that resolves within $24 \mathrm{hr}$. The potential of the model is evaluated via computational tests performed to assess the implications of neuro-endocrine activity across the host. Exploring the possible effects of systemic perturbations enables us to trace the dynamics of a systemic inflammatory response syndrome improving our understanding of how interacting inflammatory responses and neural based mechanisms influence the host's ability to regulate inflammation. Since both glucocorticoids and catecholamines are used clinically in the context of systemic inflammation the proposed modeling has the potential for direct clinical relevance. 


\subsection{A physiological model for autonomic control of heart rate in human endotoxemia}

Among the many interesting correlates for human infectious pathology arising from the human models of endotoxemia is the documentation that low-dose LPS (2-4 ng/kg BW) induces an increase in cardiac index and heart rate (Andreasen, Krabbe et al. 2008). In recent years, there is increasing recognition that elevated heart rate might be associated with increased cardiovascular mortality leading to a reappraisal of the clinical importance of heart rate as a prognostic risk factor (Palatini and Julius 1997, 2004; Palatini 2007, 2009). Being overall variations in heart rate largely dependent on autonomic modulation, an increased heart rate has been considered to reflect a diminished parasympathetic (vagal) tone and an increased sympathetic modulation of the sinus node (Lowry 2009). Such interpretation lies in agreement not only with experimental evidence indicating sympathetic activity excess (and/or parasympathetic attenuation) but also with the findings that reductions in implied vagal nerve activity is associated with increased morbidity in critically ill patients (Annane, Trabold et al. 1999). Although, the mechanism for this systemic "decomplexification" is unknown, it is likely that altered central autonomic (ANS) activity and disruptions in efferent sympathetic and parasympathetic signaling are contributory.

In this section we will discuss the potential of a physiology-based model of autonomic control of heart rate response to endotoxin, to describe changes in heart rate response to endotoxin, as a result of altered central activity, incorporating explicitly the relative contribution of efferent branches of the autonomic nervous system (sympathetic/parasympathetic outflow). Data used in this study include vital signs such as 
heart rate and parameters of heart rate variability (HRV), namely pNN50, which reflects operation of a particular modulatory reflex on the sinus node. Specifically, of particular relevance to this research are the studies where human volunteers were injected intravenously with endotoxin (CC-RE, Lot \#2) at a dose of 2-ng/kg body weight (BW) while vital signs including heart rate and parameters of heart rate variability including pNN50 were recorded (Alvarez, Katsamanis Karavidas et al. 2007; Jan, Coyle et al. 2009). In addition to clinical monitoring, blood samples are also collected and analyzed to determine hormonal and cytokine responses to human endotoxemia. The purpose of these two studies was broader and attempted to assess the influence of acute stress hormone infusion on heart rate variation under the systemic inflammatory manifestations of human endotoxemia.

Vital signals including heart rate were recorded every 30 minutes from the arterial monitoring system for the first 6 hours and then periodically taken manually for up to 24 hours after LPS administration. Specifically, heart rate was measured at $0,0.5,1,1.5,2$, $2.5,3,3.5,4,4.5,5,5.5,6$ and 24 hours in relation to endotoxin administration while the determination of HRV indices was obtained using a continuous electrocardiography technique (ECG). In a continuous ECG record, each QRS complex (resulting from sinus node depolarization) was detected and one of the time-domain measures, which analyzed in this study, included the percentage of interval differences of successive interbeat intervals greater than 50 milliseconds (pNN50). A baseline determination of this parameter was obtained every hour ( 0 to +6 hours) following endotoxin challenge and at 24 hours after LPS. This time domain statistic reflects the occurrence of large changes between adjacent heart beats and it serves as surrogate for parasympathetic influences on 
the heart (vagal function) (Goldberger, Challapalli et al. 2001). In addition to timedomain analyses, spectral methods have also been employed to describe parasympathetic modulation of the sinus node. For instance, high frequency variability (HF) $(0.15-0.4$ $\mathrm{Hz}$ ), the usual statistic for assessing respiratory sinus arrhythmia (RSA), correlates with parasympathetic and vagal tone. However, respiration rate, oftentimes, falls outside the high frequency range during the post-LPS administration and pNN50 can be used as an alternative method for assessing parasympathetic efferent activity (Berntson, Bigger et al. 1997). Further, analysis of the heart rate variability spectrum reveals lower frequency $(<0.10 \mathrm{~Hz})$ components that tend to reflect the combined effect of sympathetic and parasympathetic neural influences and as such does not reflect accurately changes in sympathetic activity (Berntson, Bigger et al. 1997; Houle and Billman 1999). Taken together, in this study among the various HRV indices, the time-domain measure pNN50 will be used to assess vagus nerve activity and parasympathetic influences on the sinus node pacemaker activity.

\subsubsection{Developing a semi-mechanistic model for the autonomic control of heart rate in acute human inflammation}

The effect on heart rate of combined modulation of sympathetic and parasympathetic (vagal) nerves has been described in a quantitative fashion since the 1930s (Rosenblueth and Simeone 1934). In the 1960s, Warner and his colleagues developed a mathematical model (Warner model) to simulate the dynamics of sinoatrial (SA) node in response to vagal and sympathetic stimulations (Warner and Cox 1962). This model consists of a set of first order differential equations that describe the release, binding and degradation of neurotransmitters in the synapses between neural fibers and the SA nodal cells. Since this 
model forms the foundation of this study, we will briefly summarize the key elements and the associated interactions. The relationship, for instance, between stimulation of sympathetic nerve to the heart and the heart rate (HR) is illustrated in Figure 6.9.

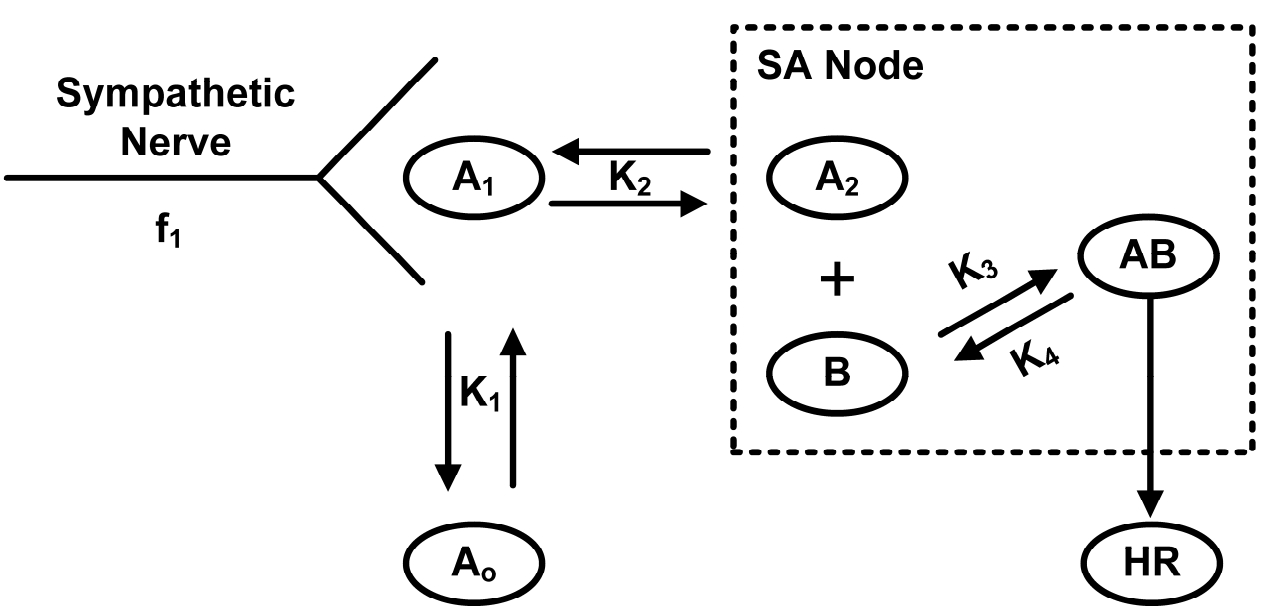

Figure 6.9: Schematic illustration of the Warner model representing the relationship between stimulation of efferent sympathetic nerve activity to the heart and heart rate (HR). $A_{1}$ represents the concentration of sympathetic neurotransmitter (catecholamine) at the nerve ending and $f_{1}$ represents the frequency stimulates preformed on the nerve; $A_{o}$ represents the concentration of catecholamine in peripheral tissues (i.e. blood); $\mathrm{A}_{2}$ represents the concentration at the active site on sinoatrial (SA) node which must react with chemical substance B to produce a change in heart rate, adapted from (Warner and Cox 1962).

Specifically, at the level of sympathetic nerve activity, the instantaneous neural activity is described by a sinusoidal function $\left(f_{1}\right)$ that serves as the input of the model stimulating the release of sympathetic neurotransmitters at the nerve ending $\left(A_{1}\right)$. However, the cardiac neurotransmitter release is not limited to centrally mediated neural traffic but may be also triggered in response to neurotransmitters from peripheral tissues such as blood (Olufsen, Tran et al. 2006). Thus, $\left(A_{0}\right)$ represents catecholamine concentration in blood which upon elevation affects the amount of SNS neurotransmitter 
released by sympathetic nerve stimulation $\left(\mathrm{A}_{1}\right)$. On the active site (SA node), an effective concentration of catecholamine $\left(\mathrm{A}_{2}\right)$ is derived from a set of first order kinetic equations that reacts with substance (B) in order to produce a change in heart rate (HR) when only sympathetic activity was present. During combined sympathetic and vagal control, albeit no interplay between the kinetics on the sympathetic and vagal sites is considered, the final heart rate is described as a function of the two instantaneous heart rates obtained from the two separate autonomic control systems.

In this study, the kinetic part of the Warner model will be used as a template for producing the concentrations of neurotransmitters in response to the related autonomic activities in human endotoxemia. Specifically, we focus on the kinetic part of the sympathetic site assuming that the released neurotransmitters from SNS nerve are triggered by the increased circulating levels of catecholamines evoked by endotoxin, Eq. (6.20), and thereby influencing the effective neurotransmitter concentration on the sinus node of the heart, equation (6.21).

$$
\begin{gathered}
\text { SNS nerve }\left\{\frac{d A_{1}}{d t}=K_{1} \cdot\left(E P I-A_{1}\right)-K_{2} \cdot\left(A_{2}-A_{1}\right)\right. \\
\text { Active SA node }\left\{\frac{d A_{2}}{d t}=K_{2} \cdot\left(A_{1}-A_{2}\right)\right.
\end{gathered}
$$

Embedding the structure of Figure 6.9 in the dynamics of our human endotoxin model, increased circulating levels of epinephrine (EPI), emerging from the dynamic evolution of the endotoxin-induced inflammatory response, stimulate the release of sympathetic neurotransmitters from the SNS nerve ending $\left(A_{1}\right)$ and are quantified by first order kinetics $\left(\mathrm{K}_{1}\right.$ and $\left.\mathrm{K}_{2}\right)$, Eq. (6.20). The re-uptake process of the SNS neurotransmitter from the sinus node to the nerve ending is described by the term $\mathrm{K}_{2}\left(\mathrm{~A}_{2}-\mathrm{A}_{1}\right)$; while the 
effective concentration of the neurotransmitter at the active site of the heart $\left(A_{2}\right)$ is influenced by the rate of sympathetic nerve traffic $\left(A_{1}\right)$, Eq. (6.21). We recognize that in the kinetic part of the Warner model sinusoidal functions were also used as input signals to sympathetic nerve stimulation. Since these functions represent the frequency stimulus which was experimentally performed in the nerves of anesthetized animals, such neural patterns are not considered in our model. Alternatively, for purposes of our model, plasma concentration of epinephrine serve as the primary "input" signal to the efferent sympathetic site, which in line with evidence (Goldstein 1981; Goldstein, McCarty et al. 1983; Grassi and Esler 1999) high plasma catecholamine concentrations are associated with high rate of sympathetic nerve traffic.

As it previously mentioned, the effective concentration $\left(A_{2}\right)$ has to react with substance (B) in order to influence the automaticity of SA nodal cells forming the signal (AB). Thus, the formed signal (AB) represents the sympathetically-mediated active signal that affects pacemaker activity and herein this mediator serves as a surrogate for the overall sympathetic response $\left(\mathrm{T}_{\mathrm{sym}}\right)$. Regarding the dynamics of the vagal site, although the relationship between the two major autonomic divisions may be highly complex, it is believed that changes in heart rate are brought about by simultaneous reciprocal changes in the autonomic influences on the heart (Glick and Braunwald 1965). Such mutual antagonism between the efferent sympathetic and parasympathetic branches of the (ANS) is further considered and quantified in equations $(6.22)-(6.23)$. 


$$
\begin{aligned}
& \text { Autonomic outflow }\left\{\begin{array}{l}
\frac{\mathrm{dT}_{\mathrm{sym}}}{\mathrm{dt}}=\frac{\mathrm{K}_{3} \cdot \mathrm{A}_{2} \cdot \mathrm{B}}{\left(1+\mathrm{k}_{\mathrm{T}_{\mathrm{sym}}, \mathrm{T}_{\mathrm{par}}} \cdot \mathrm{T}_{\mathrm{par}}\right)}-\mathrm{K}_{4} \cdot \mathrm{T}_{\mathrm{sym}} \\
\frac{\mathrm{dT}_{\mathrm{par}}}{\mathrm{dt}}=\frac{\mathrm{K}_{\mathrm{in}, \mathrm{T}_{\mathrm{par}}}}{\left(1+\mathrm{k}_{\mathrm{T}_{\mathrm{par},} \mathrm{T}_{\mathrm{sym}}} \cdot \mathrm{T}_{\mathrm{sym}}\right) \cdot \mathrm{A}_{1}}-\mathrm{K}_{\text {out }, \mathrm{T}_{\mathrm{par}}} \cdot \mathrm{T}
\end{array}\right. \\
& \mathrm{T}_{\mathrm{sym}}+\mathrm{B}=\text { constant, } \mathrm{C} \Rightarrow \mathrm{B}=\mathrm{C}-\mathrm{T}_{\text {sym }}
\end{aligned}
$$

Assuming that the two major autonomic control systems act as endogenous neuronal antagonists (Rittirsch, Flierl et al. 2008), such dynamic interaction is described by the kinetic parameters $\left(\mathrm{k}_{\text {Tsym,Tpar, }} \mathrm{k}_{\text {Tpar,Tsym }}\right)$, Eq. (6.22). Thus, the kinetics of the Warner model are extended by incorporating the stimulatory function $\left(1+\mathrm{k}_{\mathrm{Tsym}, \mathrm{Tpar}} \mathrm{T}_{\mathrm{par}}\right)$ that inhibits the first order kinetic rate $\left(\mathrm{K}_{3}\right)$ of sympathetic activity and represents the antagonism of parasympathetic response $\left(\mathrm{T}_{\mathrm{par}}\right)$. Similarly, the inhibitory effect of sympathetic response $\left(\mathrm{T}_{\mathrm{sym}}\right)$ to vagal function is represented by the linear function $\left(1+\mathrm{k}_{\mathrm{Tpar}, \mathrm{Tsym}} \mathrm{T}_{\text {sym }}\right)$ while the dynamics that define the substance $(\mathrm{B})$ are the same as in the original Warner model, Eq. (6.23).

In an effort to quantify the overall dynamics of the parasympathetic reflex activity $\left(\mathrm{T}_{\mathrm{par}}\right)$ the principles of indirect response modeling are employed (Jusko and Ko 1994; Mager, Wyska et al. 2003). The underlying assumption is that the baseline of parasympathetic tone is produced in a zero order kinetics $\left(\mathrm{K}_{\mathrm{in}, \mathrm{Tpar}}\right)$ and removed in a first order kinetics described by a constant rate $\left(\mathrm{K}_{\text {out,Tpar }}\right)$. In our endotoxin injury model, a dynamic change in vagal function $\left(\mathrm{T}_{\mathrm{par}}\right)$ is simulated due to an increase in sympathetic outflow $\left(\mathrm{T}_{\text {sym }}\right)$ which is evoked by neuroendocrine stress hormones (i.e. epinephrine) coupled with the activation of other sympathetically mediated physiological processes (i.e. blood pressure) (Davos, Davies et al. 2002) that might contribute to further 
modulation of vagal function and are represented by $\left(A_{1}\right)$ signaling mediator. The potential of the IDR modeling in simulating physiological variables (i.e. temperature) including autonomic reflex activity such as parasympathetic activity has been effectively demonstrated in (Josa, Urizar et al. 2001; Perlstein, Stepensky et al. 2001; Perlstein, Stepensky et al. 2002) and herein this modeling concept will also be explored in order to quantify heart rate dynamics as follows, Eq. (6.24).

$$
\text { Heart rate }\left\{\frac{\mathrm{dHR}}{\mathrm{dt}}=\mathrm{K}_{\mathrm{in}, \mathrm{HR}} \cdot\left(1+\mathrm{k}_{\mathrm{HR}, \mathrm{T}_{\mathrm{sym}}} \cdot \mathrm{T}_{\mathrm{sym}}\right)-\mathrm{K}_{\mathrm{out}, \mathrm{HR}} \cdot\left(1+\mathrm{k}_{\mathrm{HR}, \mathrm{T}_{\mathrm{par}}} \cdot \mathrm{T}_{\mathrm{par}}\right) \cdot \mathrm{HR}\right.
$$

The basal heart rate response is assumed to be maintained by the balance of relevant neurotransmitters that are given by a constant (zero order) rate of synthesis $\left(\mathrm{K}_{\mathrm{in}, \mathrm{HR}}\right)$ and a first order degradation rate $\left(\mathrm{K}_{\mathrm{out}, \mathrm{HR}}\right)$ (see Appendix 6.4). It is well recognized that the heart rate (HR) increases when sympathetic stimulation increases and that it decelerates upon increased parasympathetic response (Uijtdehaage and Thayer 2000). Hence, the effect of sympathetic and parasympathetic activities on the heart rate is quantified by $\left(\mathrm{k}_{\mathrm{HR}, \mathrm{Tsym}}\right)$ and $\left(\mathrm{k}_{\mathrm{HR}, \mathrm{Tpar}}\right)$, respectively. Specifically, in this model the vagus nerve mediates deceleration of heart rate by stimulating the degradation rate of heart rate response $\left(\mathrm{K}_{\mathrm{out}, \mathrm{HR}}\right)$. Taken together, in our endotoxin injury model, cardiac acceleration is induced by the antagonistic interplay of autonomic activities on the heart manifested as prevalence (increase) of efferent sympathetic activity $\left(\mathrm{T}_{\text {sym }}\right)$ and attenuation of parasympathetic nervous system function $\left(\mathrm{T}_{\mathrm{par}}\right)$. Such a semi-mechanistic, systems based modeling approach integrates central influences between autonomic control systems and physiological inflammatory components making it a critical enabler for clarifying how cellular events and inflammatory processes mediate the links between patterns of autonomic activities and clinical outcomes. 


\subsubsection{Elements of the physiology-based model of human endotoxin-induced inflammation}

We have previously demonstrated the feasibility of a multiscale physicochemical host response model that integrates essential regulatory processes across the host linking the initiating signal (LPS) with transcriptional (cellular) dynamics, signaling cascades and hormonal (physiogical) components. Specificaly, elementary pro-inflammatory patways (i.e. NF-kB signaling module) triggered by the recognition process of LPS form its signaling receptor (i.e. R, TLR4) propagate the acute inflammatory reaction on the transcriptional response level (P, A, E). Essential aspects associated with the neuroendocrine immune crosstalk and systemic "decomplexification" are also considered. In an effort to assess autonomic modulation of the sinus node of the heart, we attempted to describe the effect on heart rate of simultaneous sympathetic and vagal controls as illustrated in Figure 6.10. Specifically, at the systemic level of the sinus node, physicochemical interactions related to the release, binding and degradation of cardiac (SNS) neurotransmitters $\left(A_{1}, A_{2}\right)$ are incorporated. Such interactions are stimulated by the neuroendocrine axis and particularly by circulating levels of epinephrine (EPI) released upon endotoxin from neural SNS pathway. During the progression of endotoxin induced inflammation, disruptions in autonomic cardiac control are evaluated by alterations in the interplay between sympathetic $\left(\mathrm{T}_{\text {sym }}\right)$ and parasympathetic $\left(\mathrm{T}_{\text {par }}\right)$ arms of the autonomic nervous system which thereby influence the intrinsic pacemaker activity (heart rate). Thus, the proposed model intends to associate disordered neuroendocrine function with concomitant dysfunctional adrenergic modulation at the sinoatrial node (cardiac pacemaker). 


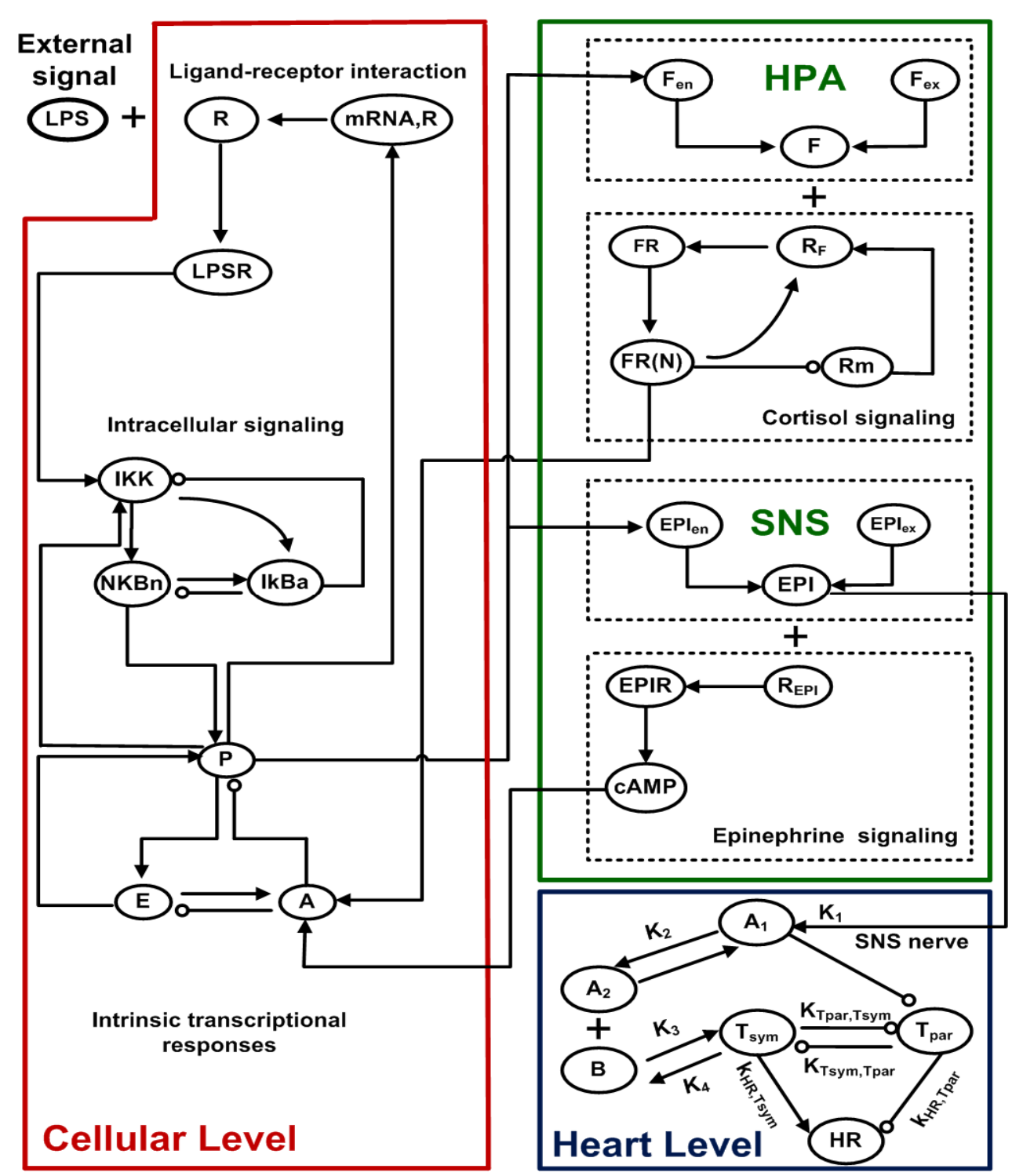

Figure 6.10: Network topology of the multiscale model of human endotoxemia for the assessment of autonomic heart rate regulation. Elementary pro-inflammatory pathways (i.e. NF-kB signaling module) triggered by the recognition process of endotoxin (LPS) from its signaling receptor (TLR4, R) propagate the effect of LPS signaling on the transcriptional (cellular) response level (P, A, E). Essential modules associated with the release of stress hormones (cortisol (F), epinephrine (EPI)) from neuroendocrine axis (HPA, SNS) coupled with their anti-inflammatory influence on the host are further considered. Finally, at the systemic level, biochemical reactions associated with the release, binding and degradation of cardiac (sympathetic) neurotransmitters $\left(\mathrm{A}_{1}, \mathrm{~A}_{2}\right)$ on the SA node are also incorporated. Efferent autonomic outflow 
is represented by sympathetic $\left(\mathrm{T}_{\text {sym }}\right)$ and parasympathetic activities $\left(\mathrm{T}_{\text {par }}\right)$ that act antagonistically giving rise to changes in heart rate (HR) response assessed by clinical monitoring of vital signs.

\subsubsection{Model calibration and validation}

Of critical importane in developing a mathematical model is the relation between the in silico model and the "real-world" process that is being modeled. As nicely discussed in (Vodovotz, Clermont et al. 2004) validation strategies are focused at two basic levels that involve the assumptions that go into the development of the model and the subsequent behavior of the model. Such strategies consist of comparing the behavior of the model with available experimental data. For instance, if the model successfully reconstructs the relevant experimental data, then it is deemed valid not only for that test but also for further assessment predicting inflammatory responses in settings on which it had not been trained explicitly (Vodovotz, Chow et al. 2006). Thus, the appropriateness of the assumptions invoked in the construction of the proposed model will be demonstrated in the following three stages including: (i) calibration of the model using human experimental data associated with heart rate measurements and time-domain measures of heart rate variability, namely pNN50 after exposure to low-dose of LPS; (ii) model verification using experimental data that have not been used as a training data set. These data refer to human subjects received either low dose (2ng/kg body weight) LPS or an infusion of epinephrine for $3 \mathrm{hr}$ before LPS administration and continued until +6 hours. Hence, we opt to assess the validity of our model by assessing the cardiovascular implications of acute epinephrine infusion on the host, and finally (iii) further qualitative model validation simulating a series of biological implications of the host response to endotoxin that can be equated with the (complex) non-linear dynamics of severe 
inflammation in the critical care setting. Such scenarios refer to systematic perturbations that modulate the dynamics towards either an irreversible response or in favor of a balanced immune response depending on the anti-inflammatory "reservoir" of the host relative to the intense (pro) inflammatory response.

\section{$\underline{\text { Parameter Estimation }}$}

Model kinetic parameters involved in the autonomic control of heart rate are estimated by minimizing the discrepancy (error) between model predictions and the experimental data as depicted in Table 6.2 (see Appendix 6.4). Relevant experimental data are normalized by taking the ratio of the measured response at each time point of the study period with respect to the control time point $(\mathrm{t}=0 \mathrm{hr})$. Thus, the associated model variables represent dimensionless entities and are considered to quantify the response of the immune function. The parameter estimation (optimization) problem consists of a nonlinear performance criterion (sum of square of errors) and is solved using MATLAB nonlinear optimization solvers such as fmincon (Contreras and Ryan 2000). All the other parameters related to the propagation of LPS signaling on the transcriptional level and to the neuro-endocrine immune system interactions are maintained to agree with those presented Table 6.1 (see Appendix 6.4). The differential equations are solved using MATLAB's solver ode15s, which is a variable-order, variable-step solver for stiff ordinary differential equations. Since it is a variable-step solver, the numerical solutions are, in general, computed at times that extent those where the experimental data were recorded. Hence, in order to obtain computed values at the specified times where experimental data were recorded, numerical interpolation is required. 
The performance of the model in reconstructing the clinical manifestations of human endotoxemia is presented in Figure 6.11. In essence, a self-limited inflammatory response, as previously simulated (Foteinou, Calvano et al. 2009a, b), involves the successful elimination of the inflammatory stimulus (endotoxin) within $2 \mathrm{hr}$ postendotoxin administration followed by subsequent resolution of all inflammatory manifestations (i.e. transcriptional responses, hormonal concentrations) within $24 \mathrm{hr}$. Herein, at the level of autonomic cardiovascular control, intravenous administration of endotoxin elicits tachycardia (elevated heart rate, HR) as a result of cardiac autonomic imbalance, reflected by increased sympathetic activity $\left(\mathrm{T}_{\text {sym }}\right)$ and reduced parasympathetic response $\left(\mathrm{T}_{\mathrm{par}}\right)$. An increase in sympathetic activity followed by reductions in implied vagal nerve activity have now been noted during inflammatory conditions associated with human endotoxemia (Lowry 2009). In our computational model, such dysregulation is mediated by acute neuroendocrine stress response evoked by endotoxin and specifically by increased circulating levels of epinephrine which give rise to a high rate of efferent sympathetic nerve traffic. Such stimulation is manifested as up-regulation in the concentration of cardiac sympathetic neurotransmitters $\left(A_{1}, A_{2}\right)$ that participate in the sympathetic control of heart rate (HR). 

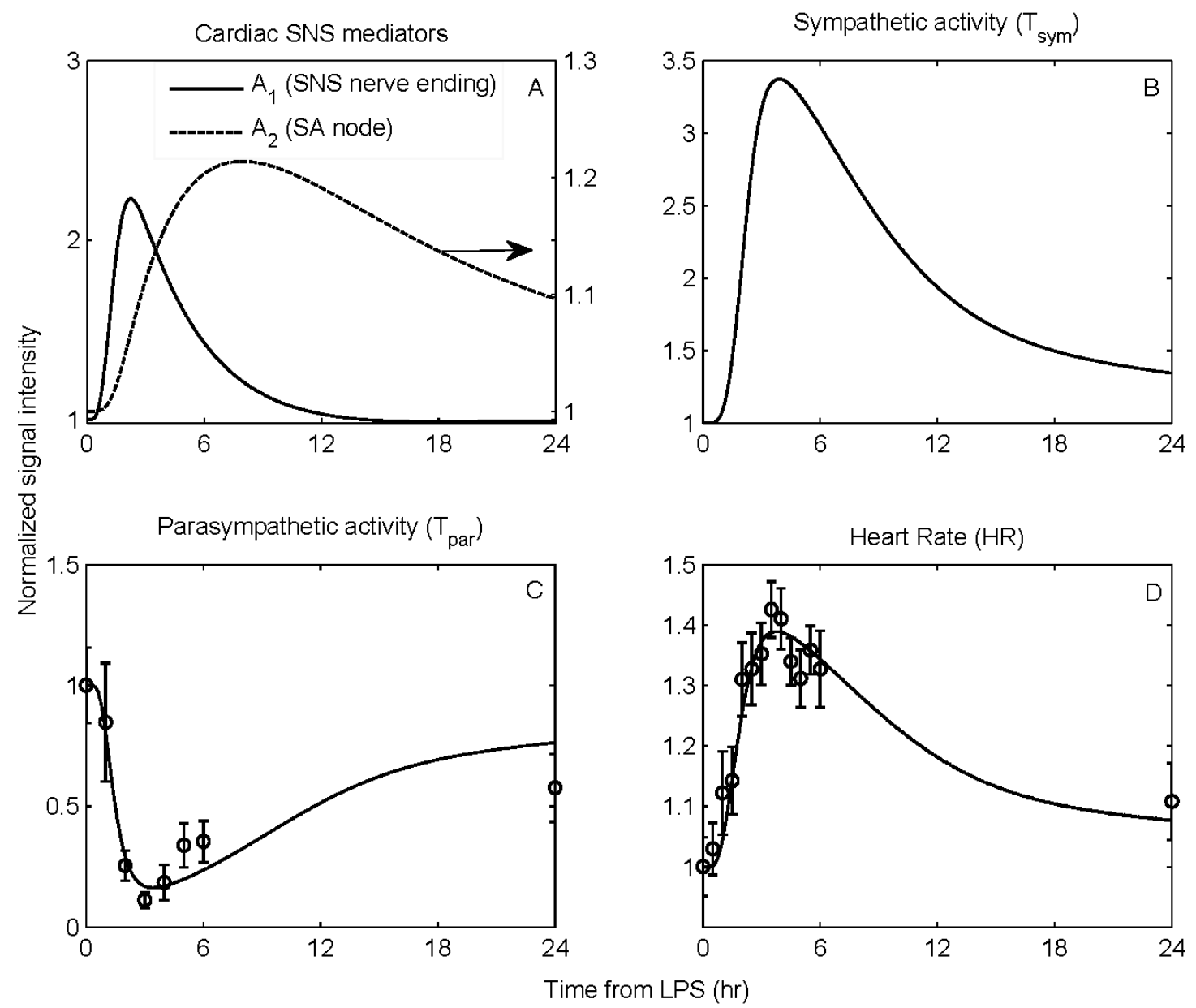

Figure 6.11: Dynamic profiles of the elements that constitute the autonomic heart rate regulation signaling module in human endotoxemia.(A) Simulated concentrations of cardiac (SNS) neurotransmitters at the level of sympathetic nerve ending $\left(\mathrm{A}_{1}\right)$ and at the active site of sinus node $(\mathrm{SA})$ of the heart $\left(\mathrm{A}_{2}\right) ;(\mathrm{B})$ Efferent sympathetic activity during the progression of the acute inflammatory reaction; (C) Simulated efferent parasympathetic (vagal) activity and (D) Heart rate (HR) response to endotoxin induced inflammation. Human experimental data (open circles) associated with vagal measurements (time domain HRV measure, pNN50) and vital signs (heart rate measurements) are used to calibrate the model. Solid lines $(-)$ represent model predictions under conditions of low-dose endotoxin while $\circ$ circles refer to experimental data expressed as mean \pm SEM. The initial condition of the inflammatory stimulus $(\operatorname{LPS}(\mathrm{t}=0 \mathrm{hr})=1)$ reflects LPS concentration relative to $2-\mathrm{ng} / \mathrm{kg}$ body weight. 
Implications of acute epinephrine infusion on the host response to endotoxin

It is well established that the SNS-driven "fight or flight response" is an essential physiological reaction activated during acute stress or illness (Padgett and Glaser 2003). Catecholamines, the main neurotrasmitters of the sympathetic nervous system pathway, exert anti-inflammatory and vasoactive properties affecting both immune cell activation and cardiovascular function (van der Poll 2000). At the cellular (immune) level, we have previously simulated the effect of epinephrine on attenuating the pro-inflammatory manifestations of human endotoxemia via a cAMP dependent mechanism. Such mechanism is stimulated by $\beta-2$ adrenergic receptors and results in potentiation in the anti-inflammatory IL-10 signaling (Foteinou, Calvano et al. 2010-in press). Although the immunosuppressive effects of antecedent periods of catecholamine excess following the systemic inflammatory manifestations of human endotoxemia have been well described (van der Poll, Coyle et al. 1996; Van der Poll and Lowry 1997), their effect on heart rate parameters induced by endotoxin is not well understood.

Predicated upon this, the influence of epinephrine (EPI) infusion initiated $3 \mathrm{hr}$ before the intravenous administration of endotoxin and continued until $+6 \mathrm{hr}$ after LPS exposure is simulated at various doses in Figure 6.12. We specifically sought to simulate whether there exists a particular dose of exogenously-induced catecholamine excess (defined in our model by the parameter, $\left.\mathrm{R}_{\mathrm{in}, \mathrm{EPI}}\right)$ that describes significantly the relevant experimental data. Thus, increasing the parameter $\left(\mathrm{R}_{\mathrm{in}, \mathrm{EPI}}\right)$ at various values the total concentration of EPI represented by dashed and dotted lines (Figure 6.12A) increases in a dose-dependent manner which subsequently potentiates the cardiac sympathetic activity (Figure 6.12B) relative to the response invoked by the administration of the inflammatory stimulus. 

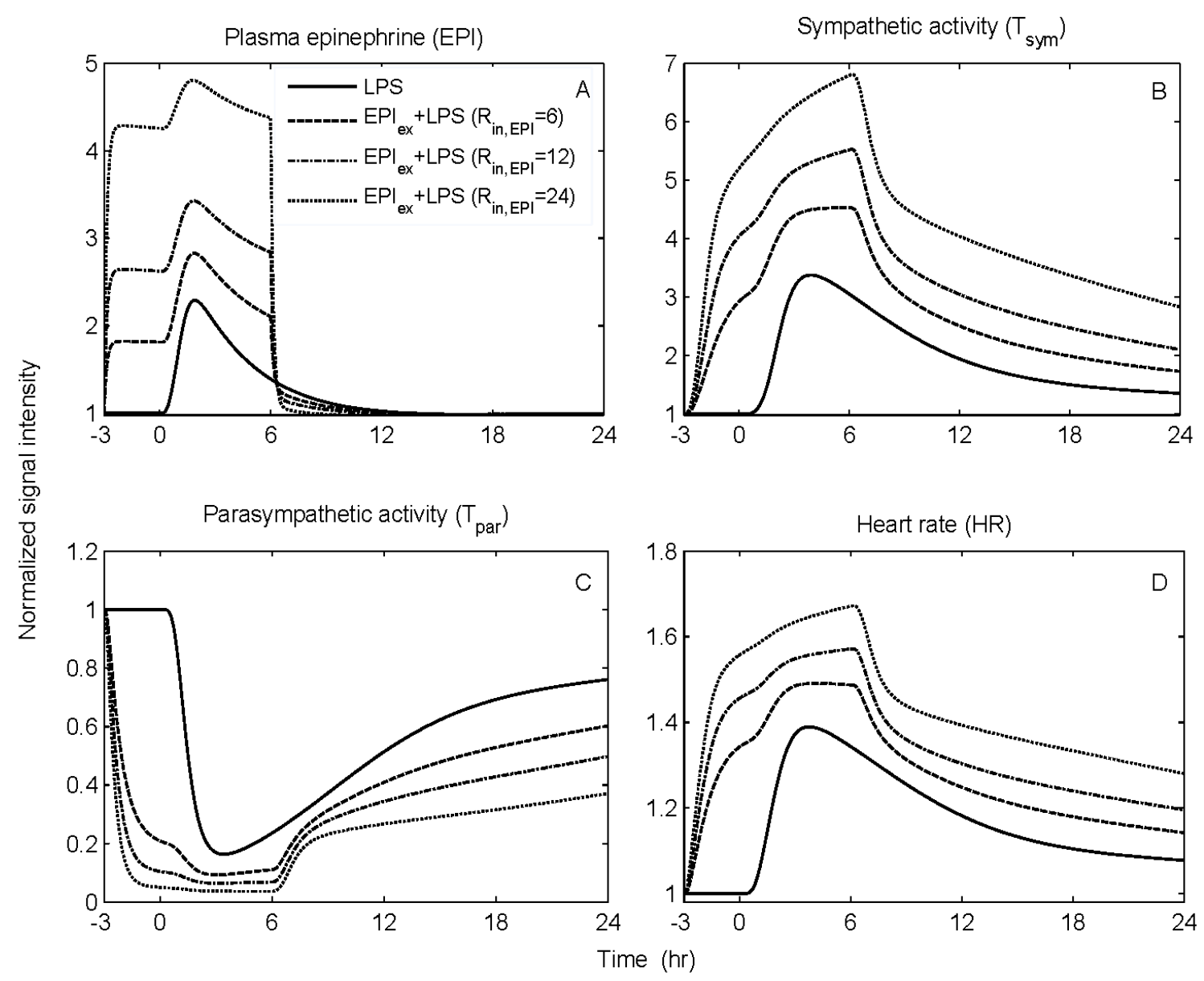

Figure 6.12: In silico simulation of the cardiovascular (sympathoexcitatory) effects of acute epinephrine infusion on the host initiated $3 \mathrm{hr}$ prior to endotoxin challenge $(\mathrm{t}=0 \mathrm{hr})$ and continued for another $6 \mathrm{hr}$ after LPS. Solid lines simulate the host dynamics under conditions of low-dose endotoxin (LPS) while dashed and dotted lines reflect the dynamics of the host pre-exposed to epinephrine infusion at various doses. The acute pre-exposure of the host into epinephrine $\left(\mathrm{w}_{\mathrm{EPI}, \mathrm{ex}}=1\right)$ at increasing values of the parameter $\mathrm{R}_{\mathrm{in}, \mathrm{EPI}}=6$, 12,24 (represented by a, b and c lines respectively) potentiates (A) circulating levels of epinephrine and (B) the overall efferent sympathetic outflow $\left(\mathrm{T}_{\mathrm{sym}}\right)$ relative to the responses induced by endotoxin administration while (C) vagal activity is significantly attenuated compared to the effect induced only by LPS and finally (D) heart rate response to endotoxin is further increased due to prior epinephrine infusion.

Such increase in efferent sympathetic outflow further diminishes the parasympathetic (vagal) function $\left(\mathrm{T}_{\mathrm{par}}\right.$ ) and thereby affects the intrinsic pacemaker activity as assessed by 
increased heart rate (HR). Experimentally, such modulation in parasympathetic and heart rate response to endotoxin under conditions of prior epinephrine infusions is demonstrated in (Jan, Coyle et al. 2009). Regarding the experimental study, antecedent EPI infusion mediated a decrease in parasympathetic function which was significantly different from the effect induced by LPS (Figure 6.13C) and a significantly higher heart rate response (HR) (Figure 6.13D). It is important to emphasize that the aforementioned experimental data represent the dynamics of the host under conditions of a particular dose of epinephrine and are used to test the validity of our model in predicting inflammatory relevant responses in situations on which it has not been trained. Although plasma concentrations of epinephrine are not available under conditions of pior EPI infusion, our simulations indicate that there exists a value of the model parameter $\left(\mathrm{R}_{\mathrm{in}, \mathrm{EPI}}\right)$ that captures the vagolytic influence of exogenously-induced catecholamine excess as assessed by an average correlation coefficient that approximates the value of 0.8 between relevant experimental data and model output (Table 6.5, see Appendix 6.4). 


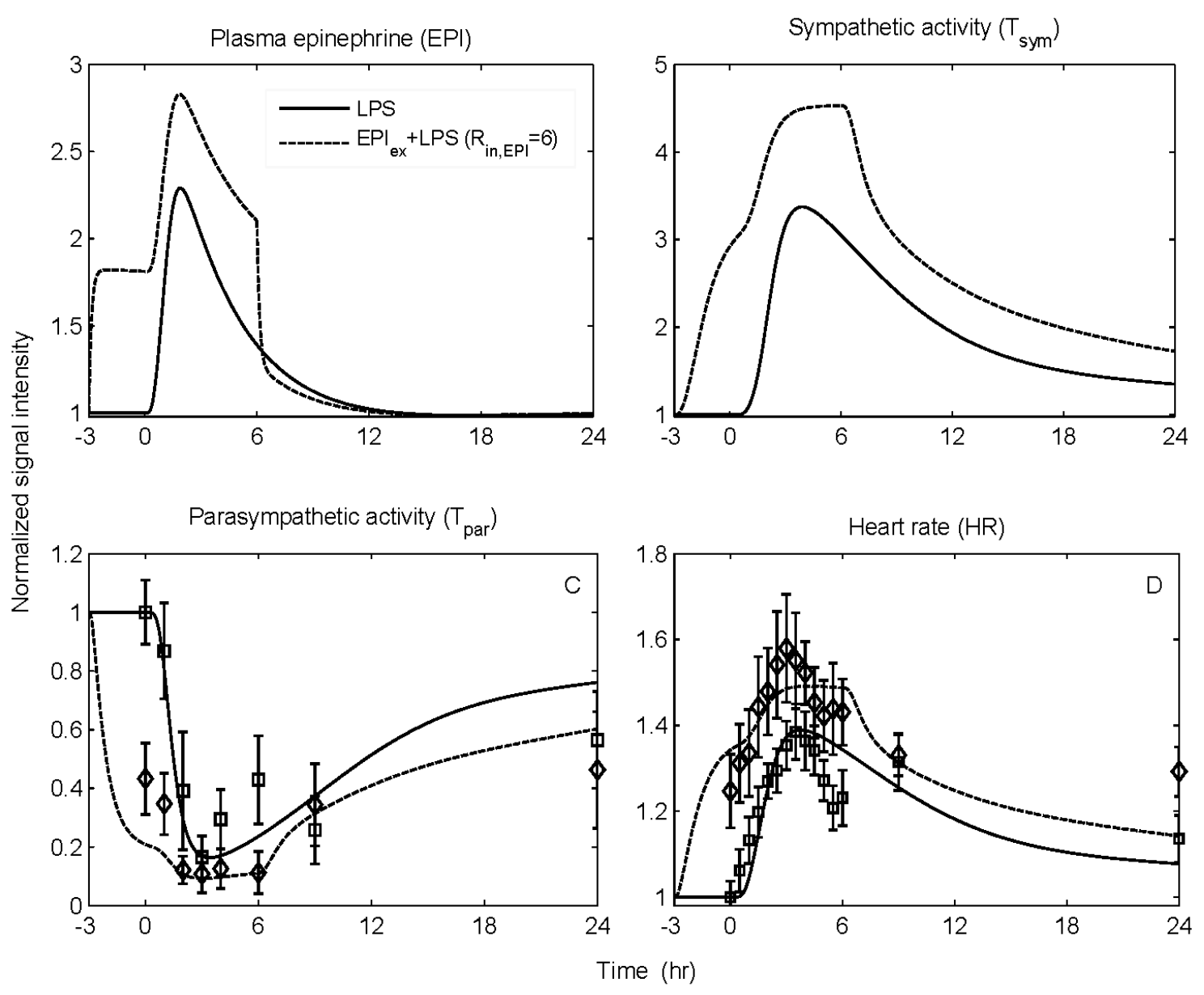

Figure 6.13: In silico assessment of the cardiovascular implications associated with acute epinephrine infusion on the host. Human experimental data depicted by squares and diamonds represent mean \pm SEM refer to human subjects that received either low dose (2ng/kg BW) LPS or an infusion of epinephrine for $3 \mathrm{hr}$ before LPS administration and continued until +6 hours, respectively. These data are specifically used to validate qualitatively the structure of the proposed human inflammation model employed from (Jan, Coyle et al. 2009) and not to train the model. Descriptive statistics in the original experimental study show that there was a significant change in the parasympathetic activity $\left(\mathrm{T}_{\mathrm{par}}\right)$ and heart rate response (HR) across the two experimental conditions ( $\square$ vs. $\diamond$ ) from $0 \mathrm{hr}$ until 24 hours after LPS exposure. Computationally such situation is captured by the differential predicted responses between dashed and solid lines. We specifically observe that there exists a simulated trajectory of exogenously induced catecholamine excess (represented by dashed lines, $\mathrm{R}_{\mathrm{in}, \mathrm{EPI}}=6$ ) that lies in general agreement with the relevant human experimental data. 
Dose-dependent effects of acute endotoxin injury on the neuroendocrine-immune axis

The dose-dependence LPS inflammatory effects on immune-endocrine host responses is reported by relevant human studies (Suffredini, Hochstein et al. 1999; Vedder, Schreiber et al. 1999). Specifically, in the study (Vedder, Schreiber et al. 1999) increasing the concentration of LPS leads to differential peak responses of the human host response as assessed by immune-neuroendocrine parameters including cytokines, stress hormones (i.e. cortisol) and physiological responses (i.e. heart rate) after the administration of low doses of endotoxin (i.e. $0.4 \mathrm{ng} / \mathrm{kg}$ ) in healthy human subjects. On the other hand, high doses of endotoxin can be responsible for a dysregulation in the host defense intrinsic dynamics, even though this bacterial byproduct does not proliferate as a Gram-negative bacteria (Munford 2006). Regarding endotoxin administration and mortality, it is generally accepted that the maximum dose of LPS that can be safely administered to humans is $4 \mathrm{ng} / \mathrm{kg} \mathrm{BW}$ (Andreasen, Krabbe et al. 2008). That the administration of a larger dose can amplify the immune host response was dramatically illustrated by a patient who injected herself with Salmonella endotoxin (Taveira da Silva, Kaulbach et al. 1993). In the following we will demonstrate the ability of our model to enable such "predictions" providing further evidence of the validity of the assumptions invoked in the development of our model.

In an effort to simulate proper responses to survivable and lethal endotoxin doses, we simply vary the concentration of LPS at time zero (LPS $(\mathrm{t}=0 \mathrm{hr}))$ carrying out simulations with low (i.e. $\operatorname{LPS}(\mathrm{t}=0 \mathrm{hr})=0.4 \mathrm{ng} / \mathrm{kg}$ ) and high LPS doses (i.e. $8 \mathrm{ng} / \mathrm{kg}$ - four times greater than the nominal value $(2 \mathrm{ng} / \mathrm{kg} \mathrm{BW})$ used to calibrate the model), (Figure 6.14 and Figure 6.15). 


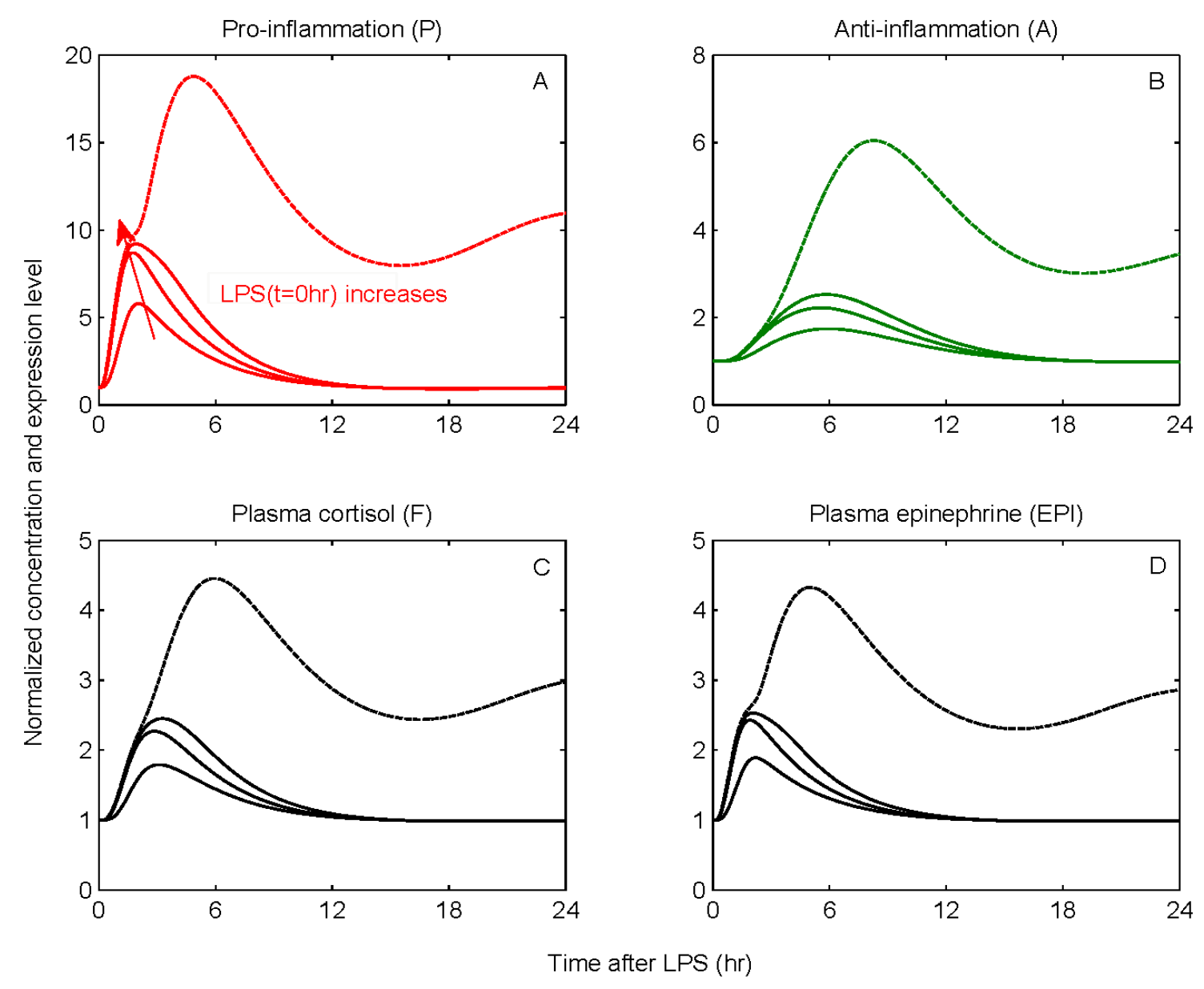

Figure 6.14: Simulated dose dependent effects of LPS on neuroendocrine immune system interactions. A high concentration of LPS can cause a dysregulation in the host dynamics characterized by abnormal transcriptional and hormonal responses. Temporal responses of critical inflammatory components for various initial conditions of the inflammatory stimulus include (A) pro-inflammatory response (P); (B) antiinflammatory response (A) and stress hormones such as (C) cortisol (F) and (D) epinephrine (EPI). Solid lines simulate the progression of a self-limited inflammatory response at increasing LPS doses (LPS(t=0hr) $=0.4 \mathrm{ng} / \mathrm{kg}, \operatorname{LPS}(\mathrm{t}=0 \mathrm{hr})=4 \mathrm{ng} / \mathrm{kg}$ and $\operatorname{LPS}(\mathrm{t}=0 \mathrm{hr})=6 \mathrm{ng} / \mathrm{kg})$ that are less than the critical threshold $(\mathrm{LPS}(\mathrm{t}=0 \mathrm{hr})=8 \mathrm{ng} / \mathrm{kg}$ - four times greater than the nominal value $(2 \mathrm{ng} / \mathrm{kg} \mathrm{BW})$ used to calibrate the model) that gives rise to unresolved inflammatory responses represented by sustained inflammatory markers and hormonal responses (dashed lines). 


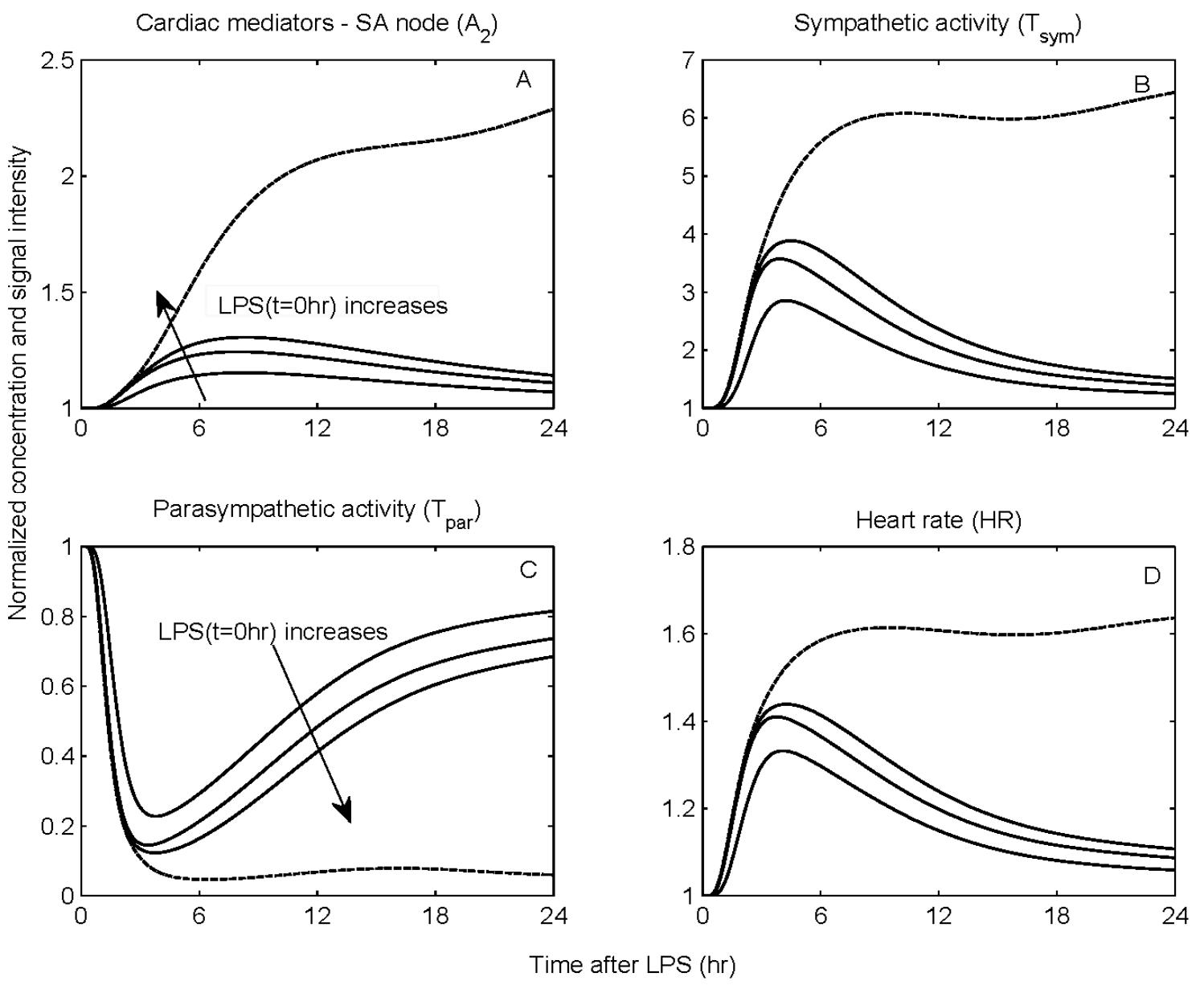

Figure 6.15: Simulated dose dependence of LPS effects on systemic (heart) level responses. A high inflammatory challenge disrupts the autonomic control systems which give rise to prolonged elevations in heart rate. Dynamic responses of $(A)$ catecholamines at the sinus node of the heart $\left(\mathrm{A}_{2}\right)$; $(\mathrm{B})$ efferent sympathetic, (C) parasympathetic activities and (D) heart rate (HR) responses to increasing levels of the inflammatory stimulus (LPS). While solid lines represent constrained inflammatory responses, dashed lines refer to a persistent inflammatory response simulated by high LPS concentration given that such situation can be equated to the severely stressed clinical phenotype manifested as sustained elevations in heart rate (cardiovascular instability).

We observe that when the concentration of LPS exceeds a critical threshold, the inflammatory response does not abate as was seen in solid lines where lower doses of LPS were simulated. This response is characterized by the uncontrolled secretion of 
endocrine hormones (cortisol, epinephrine) that are not adequate to balance (control) the overall immune response, thereby giving rise to a cytokine "burst", Figure 6.14. Such dysregulation is further accompanied by impaired autonomic function and cardiac instability manifested as sustained elevations in heart rate response, Figure 6.15. Prolonged heart rate elevations are particularly simulated due to a persistent diminished vagus nerve activity $\left(T_{\text {par }}\right)$ and/or sympathetic $\left(T_{\text {sym }}\right)$ overshooting that occur under conditions of severe endotoxin injury. In acute critical illness, comparable to the overwhelming immune response, adrenergic stress may also get out of control and cause adverse effects.

Qualitatively, such situation where the dynamic interactions between proinflammatory and late anti-inflammatory mediators may lead to a non-abating inflammatory response (trajectories of inflammatory relevant components do not return to baseline-homeostasis) can be equated with the progression of an exacerbated inflammatory state in the early phase of injury. Clinically, a sustained acute inflammatory response is manifested as septic shock that could culminate in multiple organ dysfunction. Although this might be a simplification of the pathogenesis of sepsis, this assumption is supported by the persistence of high levels of pro-inflammatory cytokines (Blackwell and Christman 1996), sustained elevations of endocrine hormones and sympathetic overstimulation (or parasympathetic withdrawal) in critically ill patients (Desborough 2000; Dunser and Hasibeder 2009; Van den Berghe 2002).

We recognize that we have previously simulated the dose-dependence of LPS effects on the cellular host response level (Foteinou, Calvano et al. 2009a, b). However, the proposed model allows us to simulate concomitant dysfunctional adrenergic modulation 
of the heart and peripheral blood leukocytes during the progression of severe human injury. Specifically, impaired neuroendocrine regulation during systemic inflammatory response syndrome contributes to disruptions in cardiovascular homeostasis phenotypically expressed as persistent elevations in heart rate that are, in many cases, associated with increased morbidity and mortality (Cook, Togni et al. 2006). Having established these non-linear responses, we now consider scenarios that involve possible reversibility in the dynamics of unremitting inflammation in response to a dynamic antiinflammatory intervention strategy.

\section{$\underline{\text { Evaluation of hormone replacement "therapy" in modulating severe acute inflammation }}$}

Properly built mathematical models of inflammation oftentimes enable several relevant asymptotically stable (AS) steady states that can be equated with self-limited ("healthy") response, sustained response with ongoing presence of the inflammatory instigator and sustained inflammation without persistent inflammatory trigger (Vodovotz, Constantine et al. 2009). A fundamental assumption of our model is the existence of two AS equilibria, which depending on the anti-inflammatory capacity of the host can represent either "recovery/self-limited" or "uncontrolled/sustained tachyarrhythmias) that might account for the transient clinical phenotype of severely stressed patients. To illustrate such scenarios, we consider the trajectory of an unconstrained response, simulated as high concentration of the initial stimulus (LPS), to serve as a surrogate for the high-risk profile of severely stressed patients. Predicated upon the fact that antecedent stress hormone excess abrogates several features of human endotoxemia (Barber, Coyle et al. 1993), anti-inflammatory intervention strategies will involve pre-exposure of the host 
into either exogenously-induced catecholamine excess (Figure 6.16) or hypercortisolemia (Figure 6.17).

Critically ill patients show a variety of endocrine (hormonal) alterations and intervening with hormone substitution could possibly contribute to the recovery of the critically ill profile (Ligtenberg, Girbes et al. 2001). Catecholamines, as potent antiinflammatory and vasoactive agents, have received increased recognition as part of "replacement" therapy in the critical care setting (Santman 1992). In order to capture such situation, antecedent periods of epinephrine (EPI) infusion following high inflammatory challenge $(t=0 \mathrm{hr})$ are simulated in Figure 6.16. We observe that acute pre-exposure of the host to catecholamine excess reverses the dynamics of the intense inflammatory reaction towards homeostasis - "recovery phase" - manifested as autonomic restoration and control of cardiovascular instability which is relevantly disturbed during critical illness (Dellinger, Levy et al. 2008). Such reversibility in the transient inflammatory phenotype of severe injury annotates the impact of dynamic antiinflammation on compromising outcome. 

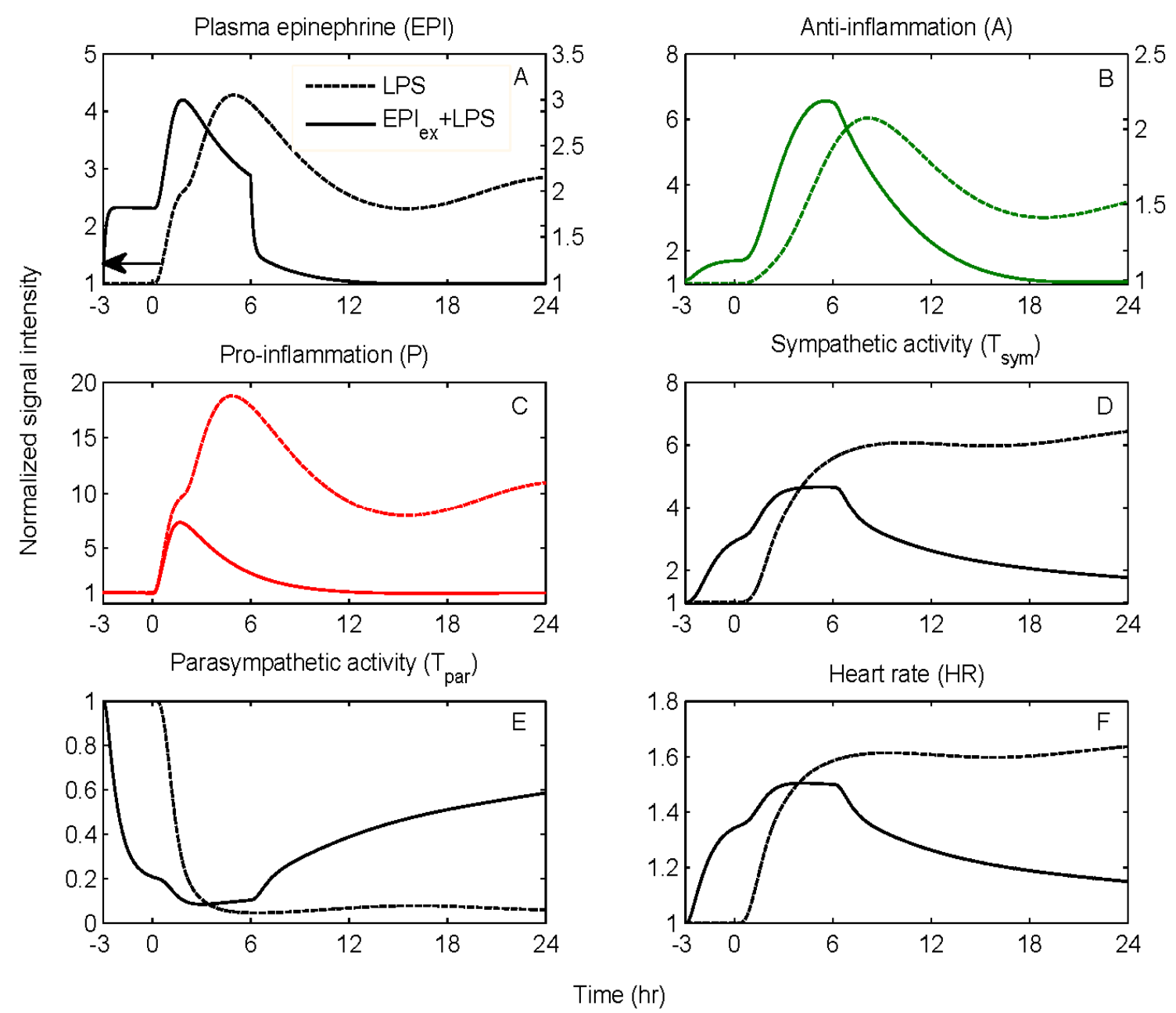

Figure 6.16: Dynamic inflammatory cellular and physiological responses as a function of time after the administration of high inflammatory challenge (dashed lines) given that at time point $0(\mathrm{t}=0 \mathrm{hr})$ solid lines represent "virtual" human subjects that receive an infusion of epinephrine. The acute pre-exposure of the host into epinephrine (initiated $3 \mathrm{hr}$ before LPS and continued until $+6 \mathrm{hr}$ after endotoxin, i.e. $\mathrm{R}_{\mathrm{in}, \mathrm{EPI}}=6$ ) attenuates the pro-inflammatory response (P), relative to the effect mediated by high concentration of LPS, via potentiation of the anti-inflammatory component of the host (A). In addition to the anti-inflammatory role of epinephrine, exogenous up-regulation in circulating levels of epinephrine increase the efferent sympathetic activity $\left(\mathrm{T}_{\text {sym }}\right)$ which is followed by further reduction in vagal function $\left(\mathrm{T}_{\text {par }}\right)$ and these autonomic changes mediate early tachycardia (HR) which is eventually restored within 24 hours. 
It is worth mentioning that the sympathomimetic properties of epinephrine prevail (Figure 6.16), during the first hours of stress hormone (EPI) infusion, i.e. 3 hours before LPS, against its anti-inflammatory effect. This prevalence is illustrated by diminished vagal function $\left(\mathrm{T}_{\text {par }}\right)$ and/or increased sympathetic control of heart rate (HR) as shown by solid lines relative to the effect invoked by LPS administration (dashed representation). However, in our model as the inflammatory response evolves the dynamic antiinflammatory mechanism (A) mediated by epinephrine signaling becomes activated and attenuates the build-up of pro-inflammation $(\mathrm{P})$ mitigating the subsequent protracted stimulation of neuroendocrine axis and the heart rate response. Such dynamics indicate the careful use of catecholamine vasopressors in the critically ill to an extent where beneficial effects still prevail without putting an excessive adrenergic stress on the heart (Dunser and Hasibeder 2006).

During the progression of sustained tachycardia (dashed lines - Figure 6.16F) attained by an irreversible disturbance (i.e. high LPS concentration), the heart rate response (HR) settles to an "unhealthy" steady state which approximates the value of 1.68 or else $107 \mathrm{bpm}$. Such simulations associate adverse stress (adrenergic) outcomes with severe cardiovascular complications manifested as persistent tachycardia at a high rate. As reviewed by Dunser et al (Dunser and Hasibeder 2009), among the several hemodynamic parameters, heart rate $>106 \mathrm{bpm}$ was linked to mortality in patients with septic shock (Azimi and Vincent 1986; Parker, Shelhamer et al. 1987). In addition to this threshold, other clinical evidence suggest that patients with persistent tachycardia $(>100$ bpm) are deemed at higher risk (Levy, White et al. 1945; Sander, Welters et al. 2005). Such strong association between increased heart rate and cardiovascular mortality has 
resurged interest in treatments that compromise heart rate control and reduce excessive adrenergic stress including hydrocortisone infusion (Dunser and Hasibeder 2009) as simulated in Figure 6.17.

Antecedent periods of hypercortisolemia participate in regulation of the hemodynamic, hormonal, and cytokine responses to infection (Briegel, Jochum et al. 2001). In severely stressed patients, low-dose hydrocortisone infusion attenuates clinical and biochemical features of the systemic inflammatory response syndrome (Briegel, Kellermann et al. 1994; Keh, Boehnke et al. 2003). This has been exclusively proven by studies evaluating human responses within the context of antecedent stress hormone excess. In healthy volunteers, glucocorticoid excess as produced by $6 \mathrm{hr}$ infusion before LPS challenge abrogates much of the clinical responses to endotoxin including heart rate (Barber, Coyle et al. 1993). It also attenuates the production of circulating proinflammatory cytokines through an increase in plasma IL-10 concentrations (van der Poll, Barber et al. 1996). Further, cortisol excess as produced by low-dose hydrocortisone infusion modulates hormonal and hemodynamic responses of human endotoxemia leading to a decrease in circulating levels of epinephrine appearance and suppressed heart rate relative to the naïve injection. In our prior models, we simulated the immunosuppressive effects of low-dose hydrocortisone infusion upon the systemic inflammatory manifestations of human endotoxemia (Foteinou, Calvano et al. 2010-in press). 

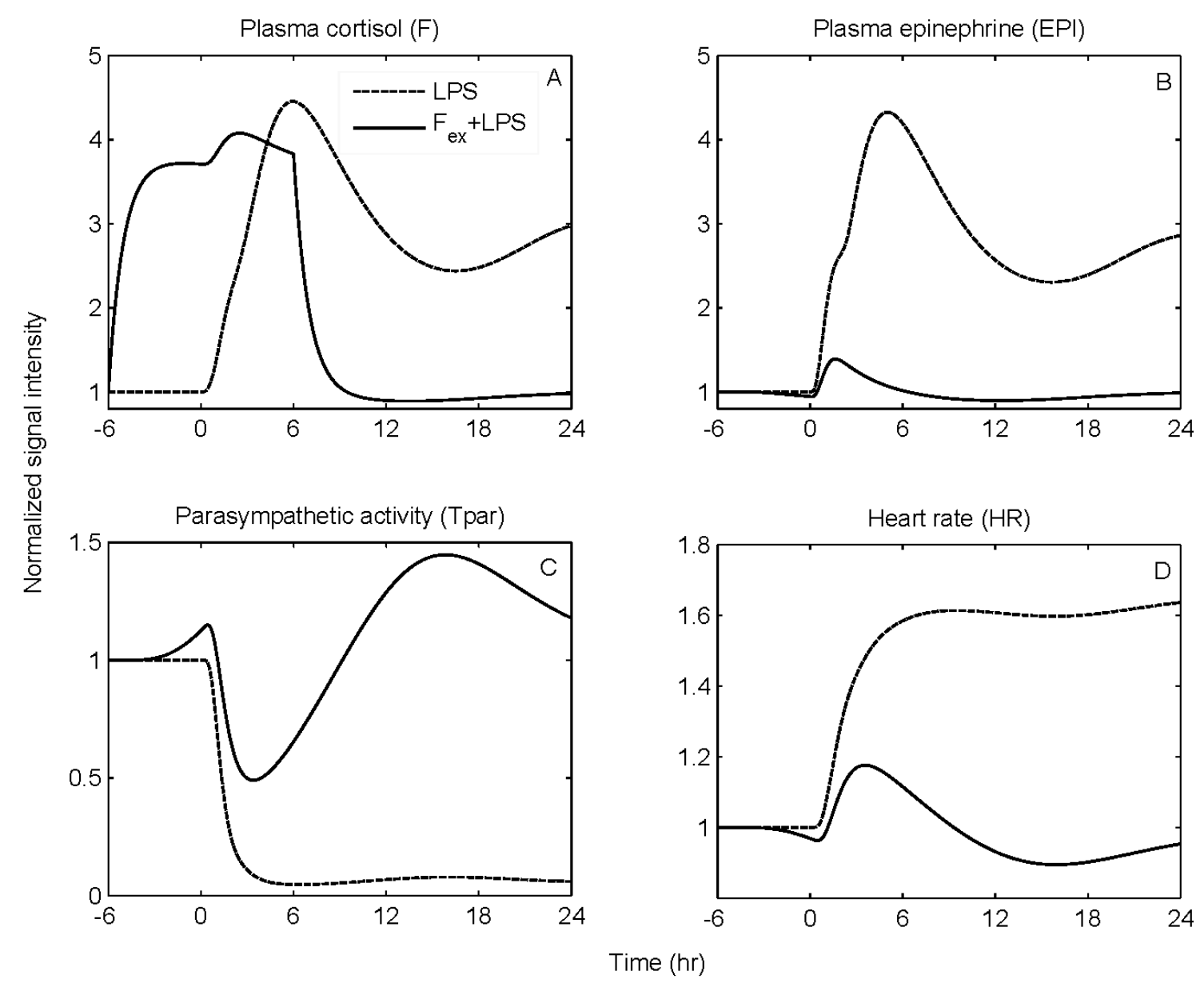

Figure 6.17: Modulation of the progression of unresolved inflammatory response due to high LPS concentration under conditions of hydrocortisone infusion. The effect of low-dose steroid administration initiated $6 \mathrm{hr}$ before high LPS concentration and continued until $+6 \mathrm{hr}$ after LPS is simulated in solid lines. Such exogenously-induced hypercortisolemia $\left(\mathrm{w}_{\mathrm{Fex}}=1, \mathrm{R}_{\mathrm{in}, \mathrm{F}}=2.922-\right.$ parameters taken from our prior work (Foteinou, Calvano et al. 2010-in press) potentiates total plasma concentration of cortisol a shown in panel (A) and modulates cytokine and hormonal responses. Circulating levels of epinephrine are attenuated in response to antecedent periods of hypercortisolemia relative to the excessive adrenergic response which is illustrated in panel (B). At the autonomic level such attenuation is expected to reduce efferent sympathetic activity mediating $(C)$ an increase in implied vagal function $\left(\mathrm{T}_{\mathrm{par}}\right)$ and finally $(\mathrm{D})$ controlling heart rate as shown by reversibility in the progression of the inflammatory reaction towards homeostasis (baseline) - "recovery phase" (solid lines). 
The aim of the present study, however, is to associate the anti-inflammatory effects of glucocorticoids, during the progression of uncontrolled inflammation, with the abrogation of prolonged and intense adrenergic stress that mitigates the subsequent amplified inflammatory response, Figure 6.17. Exogenously-induced hypercortisolemia initiated 6hr before the LPS challenge potentiates total cortisol levels (solid lines - Figure 6.17A) which accounts for alterations in the observed adrenergic stress signaling (Figure 6.17B). Such dynamic changes are simulated by reductions in circulating levels of epinephrine (EPI) that mediate further attenuation in the efferent sympathetic activity; whilst the latter gives rise to increased parasympathetic function $\left(\mathrm{T}_{\mathrm{par}}\right)$ when compared to the dynamics elicited upon the manifestation of severe endotoxin injury. Such altered adaptability in neuroendocrine and autonomic function under conditions of acute hypercortisolemia results in improved autonomic heart rate regulation as assessed by reversibility in sustained tachyarrhythmias towards homeostasis. Qualitatively, such dynamics might reflect the transient clinical improvement (i.e. "survivors") noted to critically ill patients that respond to a treatment. For example, in the observational study (Briegel, Kellermann et al. 1994) the impact of low-dose hydrocortisone infusion on modulating the course of the systemic inflammatory response syndrome is manifested by reduced heart rate, inflammatory markers and eventual recovery from stress-induced implications. However, we would like to emphasize that it is not the purpose of this study to make direct comparisons between our model predictions and clinical observations. Instead, the overall goal of this study is to develop a semi-mechanistic model of human endotoxemia as a prototype model of acute human inflammation that would potentially allow us to evaluate 
antecedent stresses upon the systemic inflammatory manifestations of acute infectious illnesses.

A key assumption of the present study is the association between increased circulating levels of epinephrine and high rate of sympathetic nerve traffic (outflow to the sinus node). Since catecholamines are secreted from sympathetic nerves, plasma catecholamines have been widely used to indicate sympathetic nervous system activity (Goldstein, Eisenhofer et al. 2003; Grassi and Esler 1999). It is thus expected any modulation in the plasma concentration of epinephrine to drive subsequent changes in the efferent sympathetic nerve activity accompanied by further changes in the autonomic heart rate dynamics. We note, however, that such relationship is not simple given that a modulation in the plasma concentration of catecholamines does not necessarily indicate a change in the rate of sympathetic nerve traffic (Goldstein, Eisenhofer et al. 2003). Recently, exogenously-induced hypercortisolemia within the context of human endotoxemia modulated inflammatory responses to low-dose endotoxin without affecting any autonomic relevant parameter including heart rate (Alvarez, Katsamanis Karavidas et al. 2007). In this experimental study, the implied discordance between acute hypercortisolemia and no modulation of adrenergic stress response to endotoxin as previously shown (Barber, Coyle et al. 1993) raises questions related to a possible nonlinear relationship between glucocorticoid activity and these inflammatory parameters. On the other hand, factors such as axonal re-uptake, local metabolism within the synaptic cleft and turnover may alter the relationship between sympathetic neural activity and plasma contents (Mueller and Ayres 1980). As new mechanisms become established and their role demonstrated reproducibly, these other mechanisms can be integrated leading to 
more complete in silico representations (Vodovotz, Clermont et al. 2004). Until all fundamental interactions and behaviors are well understood, the process of modeling remains inherently iterative. Though the current model is a very incomplete representation of all the processes involved in inflammation, we believe that it captures essential features of the multiscale nature of human acute inflammatory response that could potentially provide significant insights into how disruptions in the neuro-immune axis contribute to adverse clinical outcomes manifested as autonomic dysregulation.

In conclusion, a semi-mechanistic, physiology-based model of human endotoxemia is developed, as a prototype model of acute inflammation in humans that quantifies essential aspects of the autonomic heart rate regulation. We expanded our prior mathematical modeling work to include systemic level interactions associated with the dynamic interplay of sympathetic and parasympathetic nerves to the heart. Such physicochemical interactions are related to the release, binding and degradation of cardiac neurotransmitters that allow us to associate endogenous neuroendocrine stress responses with centrally altered autonomic activities that give rise to heart rate changes. Kinetic parameters are estimated by reconstructing human relevant experimental data associated with a constrained hyperdynamic cardiovascular response to the endotoxin paradigm. Further, the proposed model is evaluated through its potential to simulate the cardiovascular implications of acute epinephrine infusion on the host as well as a series of systematic perturbations that balance neuro-immunologic dissonance promoting inflammatory resolution and thereby cardiovascular homeostasis during the course of unremitting inflammation. 


\subsection{Appendix to the multiscale models of human endotoxemia}

\subsubsection{Table of relevant model components}

\begin{tabular}{|c|c|c|c|}
\hline Symbol & Description & Symbol & Description \\
\hline LPS & Lipopolysaccharide, endotoxin & $\mathrm{FR}(\mathrm{N})$ & $\begin{array}{l}\text { Nucler steroid-receptor } \\
\text { complex }\end{array}$ \\
\hline $\mathrm{R}$ & $\begin{array}{l}\text { Endotoxin signaling receptor } \\
\text { (TLR4) }\end{array}$ & EPI & Epinephrine \\
\hline LPSR & Endotoxin-TLR4 complex & $\mathrm{R}_{\mathrm{EPI}}$ & $\beta$ adrenergic receptor \\
\hline $\mathrm{mRNA}_{\mathrm{R}}$ & $\begin{array}{l}\text { Gene transcript of endotoxin } \\
\text { receptor (TLR4) }\end{array}$ & EPIR & $\begin{array}{l}\text { epinephrine-adrenergic } \\
\text { receptor complex }\end{array}$ \\
\hline IKK & Kinase activity & cAMP & $\begin{array}{l}\text { cyclic adenosine } \\
\text { monophosphate }\end{array}$ \\
\hline $\mathrm{NFkB}_{\mathrm{n}}$ & Nuclear concentration of NF-kB & $f_{P}$ & $\begin{array}{l}\text { efferent (cardiac) nerve } \\
\text { activity }\end{array}$ \\
\hline $\mathrm{mRNA}_{\mathrm{IkBa}}$ & $\begin{array}{l}\text { Gene transcript of NF-kB inhibitor } \\
(\mathrm{IkBa})\end{array}$ & $\mathrm{S}_{\mathrm{f}}$ & cardiac active signal \\
\hline $\mathrm{IkBa}$ & Protein inhibitor IkBa & HRV & heart rate variability \\
\hline $\mathrm{P}$ & $\begin{array}{l}\text { Transcriptional pro-inflammatory } \\
\text { response }\end{array}$ & $\mathrm{A}_{1}$ & $\begin{array}{l}\text { Catecholamines at the SNS } \\
\text { nerve ending }\end{array}$ \\
\hline A & $\begin{array}{l}\text { Transcriptional anti-inflammatory } \\
\text { response }\end{array}$ & $\mathrm{A}_{2}$ & $\begin{array}{l}\text { Catecholamines at the sinus } \\
\text { node (SA) }\end{array}$ \\
\hline $\mathrm{E}$ & Transcriptional energetic response & $\mathrm{B}$ & $\begin{array}{l}\text { Chemical substance at the SA } \\
\text { of the heart }\end{array}$ \\
\hline $\mathrm{F}$ & Cortisol & $\mathrm{T}_{\text {sym }}$ & Efferent sympathetic activity \\
\hline $\mathrm{R}_{\mathrm{m}}$ & $\begin{array}{l}\text { Gene transcript of glucocorticoid } \\
\text { receptor }\end{array}$ & $\mathrm{T}_{\mathrm{par}}$ & $\begin{array}{l}\text { Efferent parasympathetic } \\
\text { activity }\end{array}$ \\
\hline $\mathrm{R}_{\mathrm{F}}$ & $\begin{array}{l}\text { Free cytosolic glucocorticoid } \\
\text { receptor }\end{array}$ & HR & Heart rate \\
\hline FR & $\begin{array}{l}\text { Cytosolic steroid-receptor } \\
\text { complex }\end{array}$ & & \\
\hline
\end{tabular}




\subsubsection{Estimated parameter values}

Table 6.1: Estimated values of parameters involved in the neuro-endocrine immune axis.

\begin{tabular}{|c|c|c|}
\hline $\mathrm{R}_{\mathrm{in}, \mathrm{F}}\left(\mathrm{w}_{\mathrm{Fex}}=0\right)=0$ & $\mathrm{k}_{2, \mathrm{REPI}}=5.465$ & $\mathrm{k}_{\mathrm{REPI}}^{0}=11.011$ \\
\hline $\mathrm{K}_{\mathrm{in}, \mathrm{Fen}}=0.842$ & $\mathrm{k}_{3, \mathrm{REPI}}=5.546$ & $\mathrm{~K}_{\text {out }, \mathrm{A}}=0.809$ \\
\hline $\mathrm{k}_{\mathrm{Fen}, \mathrm{P}}=0.256$ & $\tau=0.053$ & $\mathrm{w}=10$ \\
\hline $\mathrm{K}_{\mathrm{out}, \mathrm{F}}=1.058$ & $\mathrm{n}=5.509$ & $\tau_{\mathrm{S}}=0.723$ \\
\hline $\mathrm{R}_{\mathrm{in}, \mathrm{F}}\left(\mathrm{w}_{\mathrm{Fex}}=1\right)=2.922$ & $\mathrm{~K}_{\mathrm{in}, \mathrm{A}}=0.461$ & $\mathrm{n}_{\mathrm{S}}=1.185$ \\
\hline $\mathrm{K}_{\mathrm{in}, \mathrm{EPI}}=5.921$ & $\mathrm{k}_{\mathrm{A}, \mathrm{cAMP}}=0.145$ & $\mathrm{~K}_{\mathrm{in}, \mathrm{HRV}}=0.038$ \\
\hline $\mathrm{K}_{\mathrm{EPI}, \mathrm{P}}=0.231$ & $\mathrm{k}_{\mathrm{A}, \mathrm{E}}=0.534$ & $\mathrm{~K}_{\text {out } \mathrm{HRV}}=0.038$ \\
\hline $\mathrm{k}_{1, \mathrm{REPI}}=3.005$ & $\mathrm{k}_{\mathrm{A}, \mathrm{FRN}}=0.401$ & $\mathrm{k}_{\mathrm{HRV}, \mathrm{S}}=35.254$ \\
\hline $\mathrm{k}_{\mathrm{R}, \mathrm{EPI}}=0.845$ & $\mathrm{~K}_{\mathrm{out}, \mathrm{EPI}}=7.286$ & $\mathrm{~K}_{\text {out }, \mathrm{EPI}}=7.286$ \\
\hline
\end{tabular}

Table 6.2: Estimated values of parameters related to the autonomic heart rate regulation.

\begin{tabular}{|c|c|}
\hline $\mathrm{K}_{1}=3.653$ & $\mathrm{k}_{\text {Tpar,Tsym }}=9.756$ \\
\hline $\mathrm{K}_{2}=0.055$ & $\mathrm{~K}_{\text {out,Tpar }}=4.200$ \\
\hline $\mathrm{K}_{3}=2.926$ & $\mathrm{~K}_{\mathrm{in}, \mathrm{HR}}=23.279$ \\
\hline $\mathrm{C}=11.286$ & $\mathrm{k}_{\mathrm{HR}, \mathrm{Tsym}}=0.055$ \\
\hline $\mathrm{K}_{\text {Tsym,Tpar }}=7.763$ & $\mathrm{k}_{\mathrm{HR}, \mathrm{Tpar}}=0.296$ \\
\hline $\mathrm{K}_{4}=3.435$ & $\mathrm{~K}_{\mathrm{out}, \mathrm{HR}}=18.942$ \\
\hline $\mathrm{K}_{\mathrm{in}, \mathrm{Tpar}}=45.181$ & \\
\hline
\end{tabular}


6.4.3 Steady-state baseline equations

\begin{tabular}{|c|c|}
\hline Eq. (6.12) & $\left\{\begin{aligned} \frac{\mathrm{dA}}{\mathrm{dt}}=0 \rightarrow \mathrm{K}_{\text {out }, \mathrm{A}} & =\frac{\mathrm{K}_{\mathrm{in}, \mathrm{A}} \cdot\left(1+\mathrm{k}_{\mathrm{A}, \mathrm{CAMP}} \cdot \mathrm{cAMP}(0)\right) \cdot\left(1+\mathrm{k}_{\mathrm{A}, \mathrm{FRN}} \cdot \mathrm{FR}(\mathrm{N})(0)\right) \cdot\left(1+\mathrm{k}_{\mathrm{A}, \mathrm{E}} \cdot \mathrm{E}(0)\right)}{\mathrm{A}(0)} \\
\mathrm{K}_{\text {out } \mathrm{A}}= & \mathrm{K}_{\mathrm{in}, \mathrm{A}} \cdot\left(1+\mathrm{k}_{\mathrm{A}, \mathrm{AMMP}}\right) \cdot\left(1+\mathrm{k}_{\mathrm{A}, \mathrm{E}}\right)\end{aligned}\right.$ \\
\hline Eq. (6.17) & 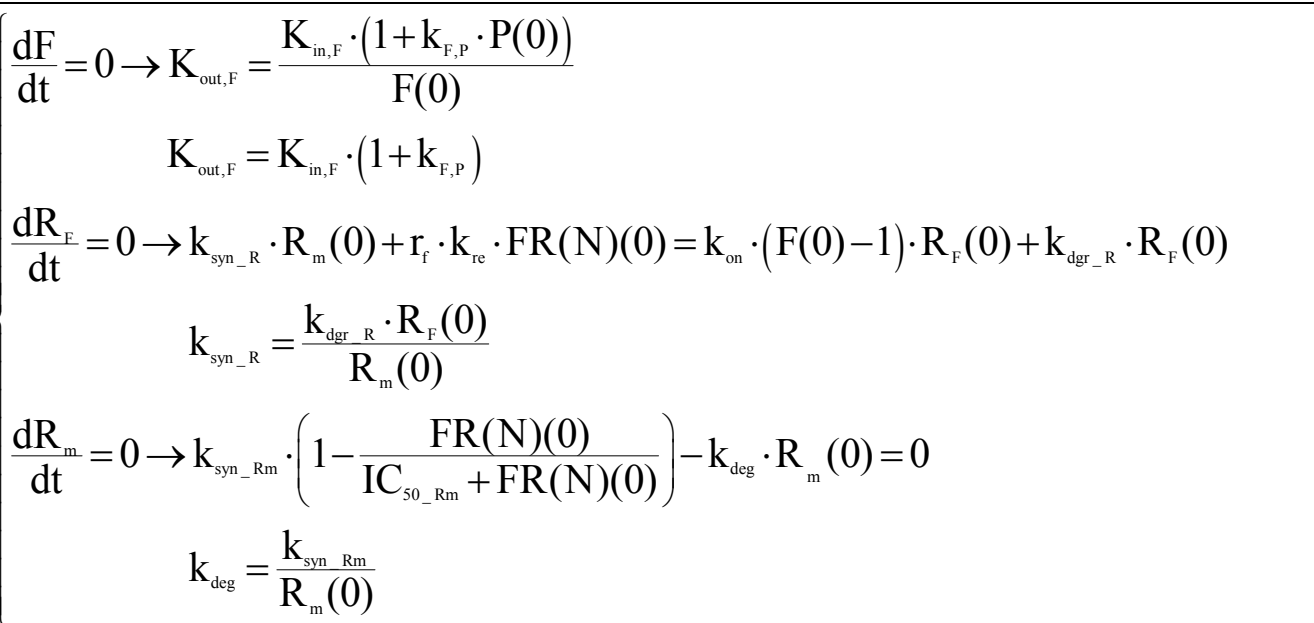 \\
\hline Eq. (6.18) & 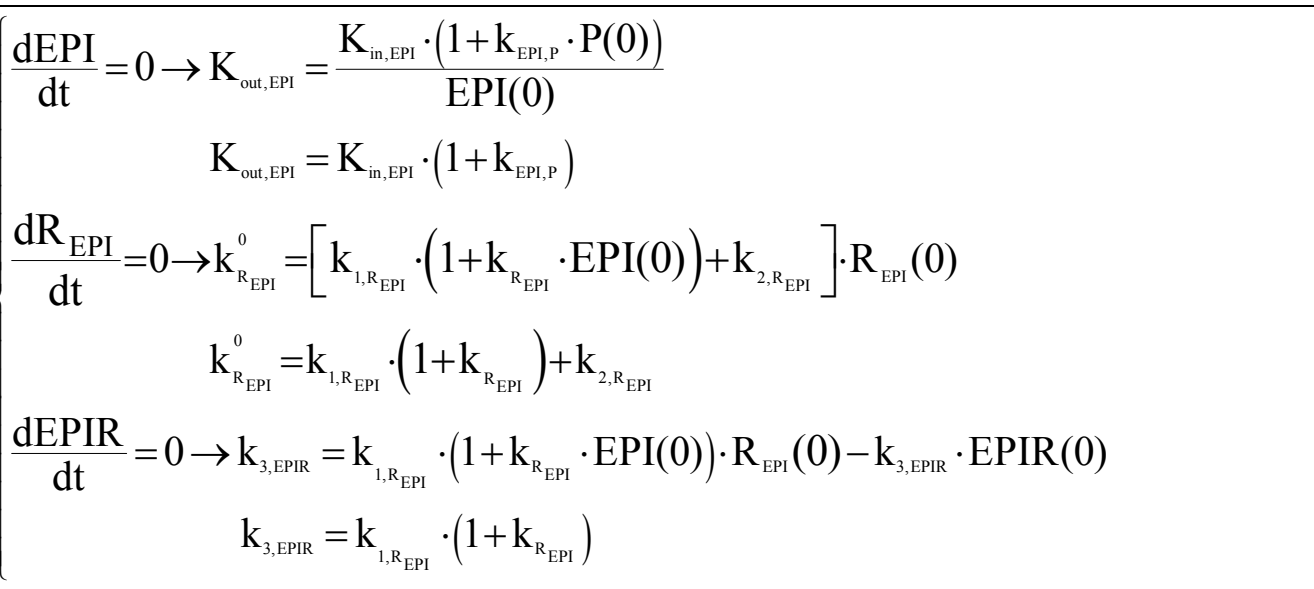 \\
\hline Eq. (6.19) & $\left\{\begin{aligned} \frac{\mathrm{dHRV}}{\mathrm{dt}}=0 \rightarrow \mathrm{K}_{\mathrm{in}, \mathrm{HRV}} & =\mathrm{K}_{\mathrm{out}, \mathrm{HRV}} \cdot\left(1+\mathrm{k}_{\mathrm{HRV}, \mathrm{S}} \cdot \mathrm{S}_{\mathrm{f}}(0)\right) \cdot \mathrm{HRV}(0) \\
\mathrm{K}_{\mathrm{in}, \mathrm{HRV}} & =\mathrm{K}_{\mathrm{out}, \mathrm{HRV}}\end{aligned}\right.$ \\
\hline
\end{tabular}




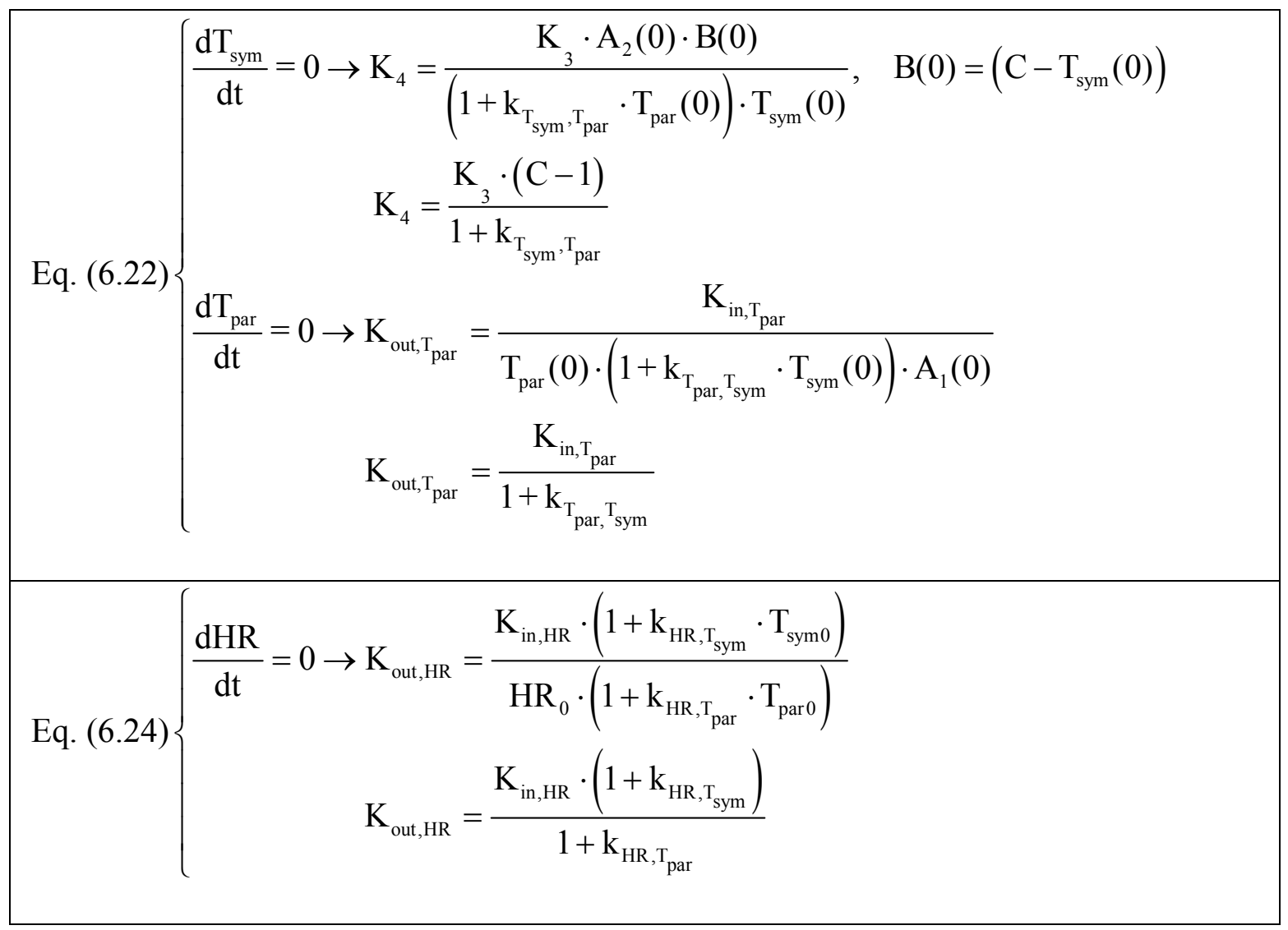

Table 6.3: Initial conditions of relevant model components

\begin{tabular}{|c|c|c|c|c|}
\hline $\mathrm{LPS}(0)=1$ & $\mathrm{mRNA}_{\mathrm{IkBa}}(0)=1$ & $\mathrm{R}_{\mathrm{m}}(0)=25.8$ & $\mathrm{EPIR}(0)=0$ & $\mathrm{~A}_{2}(0)=1$ \\
\hline $\mathrm{R}(0)=1$ & $\mathrm{IkBa}(0)=0$ & $\mathrm{R}_{\mathrm{F}}(0)=540.7$ & $\mathrm{cAMP}(0)=1$ & $\mathrm{~T}_{\mathrm{sym}}(0)=1$ \\
\hline $\mathrm{LPSR}(0)=0$ & $\mathrm{P}(0)=1$ & $\mathrm{FR}(0)=0$ & $\mathrm{f}_{\mathrm{P}}(0)=1$ & $\mathrm{~T}_{\mathrm{par}}(0)=1$ \\
\hline $\mathrm{mRNA}, \mathrm{R}(0)=1$ & $\mathrm{~A}(0)=1$ & $\mathrm{FR}(\mathrm{N})(0)=0$ & $\mathrm{~S}_{\mathrm{f}}(0)=0$ & $\mathrm{HR}(0)=1$ \\
\hline $\mathrm{IKK}(0)=0$ & $\mathrm{E}(0)=1$ & $\mathrm{EPI}(0)=1$ & $\mathrm{HRV}(0)=1$ & \\
\hline $\mathrm{NFkBn}(0)=0$ & $\mathrm{~F}(0)=1$ & $\mathrm{R}_{\mathrm{EPI}}(0)=1$ & $\mathrm{~A}_{1}(0)=1$ & \\
\hline
\end{tabular}




\subsubsection{Statistical complement}

The performance of the model in capturing the characteristics of actual measurements is assessed by measures of goodness of fit including estimation of a correlation coefficient between the data and the model as previously proposed (Chow, Clermont et al. 2005). We test the null hypothesis that the correlation coefficient between experimental data and calculated values is zero, versus the alternative that it is greater than zero yielding a pvalue quantifying the likelihood of getting a correlation as large as the one calculated by chance. If the p-value is small, for example less than 0.05 (a commonly used threshold for significance level), then the correlation is significant at $95 \%$ confidence interval and the model accurately captures the characteristics of the data. The correlation coefficients between experimental data and the simulated HRV values (depicted in Figure 6.8D) were calculated across the two experimental scenarios (LPS, EPI-3hr+LPS) as illustrated in Table 6.4. This analysis yields very small p-values indicating a strong correlation between actual measurements and the model output.

Table 6.4: Statistical assessment of the model in comparison to HRV data presented in Figure 6.8D

\begin{tabular}{|c|c|c|}
\hline Experimental scenario & $\begin{array}{c}\text { Measure of goodness of fit } \\
\text { (correlation coefficient) }\end{array}$ & p-value \\
\hline LPS (Figure 6.8D) & 0.85 & 0.0072 \\
\hline EPI-3hr+LPS (Figure 6.8D) & 0.92 & 0.0014 \\
\hline
\end{tabular}

It is important to note that the data displayed in Figure 8D (both solid and open markers), albeit well described by our model (Table 6.4), are not used to calibrate our model but 
rather to validate the intended structure of our model in predicting the non-linear interaction between peripheral immune responses and autonomic activities. Regarding the fitting process, relevant quantitative (HRV) data are employed from the experimental study (Alvarez, Katsamanis Karavidas et al. 2007) as shown in Figure 2(manuscript, last panel)) and the measure of goodness of fit yields a correlation coefficient of 0.91 and a pvalue of $0.0018(<<0.05)$. Across all experimental data used in this study (Figure 6.3) under the administration of LPS, we obtain an average correlation coefficient of 0.956 indicating significant correlation between the experimental measurements (solid markers of A, F, EPI, HRV) and the predicted values of our model.

The correlation coefficients between experimental data not used to calibrate the model and the simulated $T_{\text {par }}$ and HR values represented by dashed lines (Figure 6.13) were calculated across the two experimental scenarios (LPS, $\mathrm{EPI}_{\mathrm{ex}}+\mathrm{LPS}$ ) as illustrated in Table 6.5. This analysis yields a significant strong correlation between actual measurements and the model output.

Table 6.5: Statistical assessment of the model in comparison to data presented in Figure 6.13C and Figure 6.13D

\begin{tabular}{|c|c|c|}
\hline Experimental scenario & $\begin{array}{c}\text { Measure of goodness of fit } \\
\text { (correlation coefficient) }\end{array}$ & p-value \\
\hline LPS (Figure 6.13C) & 0.92 & 0.012 \\
\hline LPS (Figure 6.13D) & 0.89 & $1 . \mathrm{e}-4$ \\
\hline EPI $_{\text {ex }}+$ LPS (Figure 6.13C) & 0.77 & 0.023 \\
\hline EPI $_{\text {ex }}+$ LPS (Figure 6.13D) & 0.76 & $9 . \mathrm{e}-3$ \\
\hline
\end{tabular}


It is important to note that the data displayed in Figure 6.13C and Figure 6.13D (square and diamond markers), albeit well described by our model yielding a correlation coefficient $\sim 0.8$ (Table 6.5), are not used to calibrate our model but rather to validate the intended structure of our model in predicting the sympathomimetic properties of acute epinephrine infusion upon the inflammatory manifestations of human endotoxemia. Relevant quantitative data ( $\mathrm{T}_{\mathrm{par}}$ and $\mathrm{HR}$ ) are employed from the experimental study (Alvarez, Katsamanis Karavidas et al. 2007) (depicted in Figure 6.11C and Figure 6.11D) and the measure of goodness of fit yields a correlation coefficient of 0.92 for $T_{\text {par }}$ dynamics (p-value 0.012 ) and 0.89 for HR (p-value 1.e-4) indicating strong correlation between the experimental measurements and the predicted (estimated) values of the proposed model. 


\section{Chapter 7}

\section{Conclusions and Future Perspectives}

\subsection{Summary}

A critical goal of translational research is to convert novel insights from basic science to clinically relevant actions related to disease prevention and diagnosis, eventually enabling physicians to identify and evaluate treatment strategies. Integrated initiatives are identified as valuable in uncovering the mechanisms underpinning the progression of human diseases. The advent of high-throughput technologies has enabled the generation of massive amounts of biological data at an unprecedented rate, facilitating a dramatic increase in the degree of quantification applied to modern biological research (Beard, Bassingthwaighte et al. 2005). Further, the organization, regulation and dynamical responses of biological systems are in many cases too complex to allow intuitive predictions and require the development of mathematical modeling for quantitative assessments and a reliable understanding of the systems' functions. The recognition that biological systems display emergent properties has renewed interest in systems biology approaches which investigate how the various components are organized across scales and how they interact to generate a behavior. However, central to integrative systems biology is the identification of those critical components and the rules that define their interactions and give rise to the emergent host response.

In this dissertation we discussed the potential role of systems-based approaches can have in the quest to better understand and module critical physiological responses, 
namely human inflammation. We demonstrated how data analysis can yield significant insights and enable the development of semi-mechanistic, quantitative models of inflammation that can be used as minimal representations of biological reality to reconcile observations and potentially guide future experimental design. The unifying hypothesis is that the observed response is the outcome of the orchestrated interactions of critical modules in the form of a network. Thus, a multiscale integrative approach is proposed and developed that aims at exploring the emergence of interaction networks and functional modules linking processes that span various scales from the cellular (low) level to the systemic (high) host response level.

The overarching goals of this research are to demonstrate the feasibility of a relevant, physiology-based and multiscale model of human endotoxemia, as a prototype model of acute inflammation in humans, coupling essential regulatory processes across the host from the cellular to the systemic level; and to propose a template for multiscale modeling extendable to a variety of clinically important conditions. The unique aspects of the proposed modeling effort include: (i) identification of the essential responses characterizing the cellular (leukocyte) transcriptional dynamics in response to endotoxin administration. Specifically, these responses, in the case of transient human endotoxemia, include a pro-inflammatory response $(\mathrm{P})$ that consists of the early increased expression of cytokines and chemokines; an anti-inflammatory response (A) which serves as the immunoregulatory arm of the host defense system and, an energetic response (E) that involves the decreased expression of genes participating in cellular bio-energetic processes; (ii) reverse engineering of quantifiable representations of these elements exploring the concept of physicochemical (Aldridge, Burke et al. 2006) and indirect 
response (IDR) modeling (Jusko and Ko 1994) that connect extracellular signals and intracellular signaling cascades leading to the emergent transcriptional dynamics. Physicochemical modeling seeks to describe essential biological processes in terms of equations that can be physiologically interpretable. Such models work best with regulatory processes where components (i.e. "pathway signals") and connectivity are relatively well established. By incorporating biological information in the form of kinetic rules and signaling cascades we would probably be able to develop dynamic repositories of interpretable knowledge. Accordingly, in our injury model, the response is triggered by the activation of critical pro-inflammatory signaling cascades (i.e. NF-kB signaling module) as a result of the formation of an activating signal associated with the recognition of extracellular signals (LPS) from their appropriate receptors (R, TLR4). On the other hand, when prior knowledge is sparse, the manifestation of a perturbation is difficult to be explicitly described using elementary kinetic reactions. Our inability to precisely model such signaling events makes IDR appealing. The underlying assumption of indirect response models is that external signals affect indirectly the synthesis and/or degradation term of the response of interest, in our case the transcriptional dynamics; and finally (iii) multiscale, physiology-based modeling that quantifies critical aspects of the complex neuroendocrine-immune crosstalk and autonomic heart rate regulation while systemic disruptions are assessed by clinical monitoring of vital signs (i.e heart rate) and parameters of heart rate variability.

This research is accomplished by exploring the interaction networks at the level of intracellular signaling in conjunction with the (high) level of interacting hormonal and physiological components. At the cellular level, elementary signaling pathways propagate 
extracellular signals to the transcriptional response level. At the autonomic level, essential modules associated with the secretion of endocrine stress hormones (cortisol, epinephrine) are considered. Finally, at the (high) systemic level, phenotypic expressions such as clinical heart rate measurements and parameters of heart rate variability are incorporated to assess systemic abnormalities and autonomic dysfunction indicative of the severity of the host. These elements are critical for the development of a multiscale human inflammation model that bridges the initiating signal (LPS) and macroscopic (phenotypic) observables (HR, HRV) through semi-mechanistic based models that include transcriptional dynamics, signaling and hormonal components.

Model parameters are appropriately evaluated based on human experimental data that span multiple scales including gene expression data, data at the level of circulating hormones and finally data at the level of the host (i.e. clinical observables). The validity of the proposed model is demonstrated through its potential to reproduce key inflammatory relevant scenarios indicative of the complex (non-linear) dynamics of acute inflammation and involve: (1) a constrained inflammatory response elicited by low-dose endotoxin corresponding to successful resolution of all systemic inflammatory manifestation within $24 \mathrm{hr}$ after the administration of the inflammatory stimulus (endotoxin, LPS); (2) an unconstrained inflammatory response that can be elicited under high concentrations of LPS characterized by a pro-inflammatory cytokine "storm" that contributes to derangements in neuro-endocrine and autonomic activities phenotypically expressed as uncontrolled/sustained tachyarrhythmias and/or diminished physiologic variability; (3) sustained inflammatory response with ongoing presence of the inflammatory instigator - persistent infectious response where the inflammatory stimulus 
is not cleared from the host; (4) tolerance and potentiation effects evoked by the repeated pre-exposure of the host to controlled levels of endotoxin emphasizing timing and dosing as key determinants for endotoxin hypo-responsiveness or "lethality" and finally (5) systematic perturbations associated with the cardiovascular implications of acute stress hormone infusion (cortisol, catecholamine excess) on the host under conditions of either low or high infectious challenge.

A fundamental assumption of our model is the existence of two steady states that depending on the anti-inflammatory "reservoir" of the host can represent either "recovery/self-limited" or "uncontrolled/sustained inflammation" that might account for the transient clinical phenotype of severely stressed patients. However, we would like to point out that a direct comparison between our model predictions and clinical observations is beyond the scope of this dissertation. Instead, the overall goal of this research is to develop an in silico model of human endotoxemia that would allow us to evaluate antecedent stresses upon the systemic inflammatory manifestations of acute injury. Thus, the proposed model lays the foundation for a translational systems-based model of inflammation that could clarify how cellular inflammatory processes and neural-based pathways mediate the links between patterns of autonomic control and clinical outcomes. It is therefore the goal of this research to explore the possibility of developing such relevant human inflammation models that would allow us to evaluate antecedent stresses upon the systemic inflammatory manifestations of acute illnesses; thereby enabling the use of systems biology in translational research. 


\subsection{Future work}

One of the key hypotheses made in the development of the model as presented in Section 6.2 is that changes in heart rate variability (HRV) during inflammation occur due to conversion of the neuronal firing rate, represented by $f_{P}$, into neuromediator concentrations $\left(\mathrm{S}_{\mathrm{f}}\right)$ which affect changes in pacemaker cycle length driving beat to beat variations as quantified by HRV (Zaza and Lombardi 2001). For purposes of this model, the dynamics of the firing rate, $\mathrm{f}_{\mathrm{P}}$, are simulated as an exponential decay that would biologically reflect an instantaneous "burst" of efferent nerve activity. However, this time-dependent function $\left(f_{P}\right)$ should be reset to its baseline and give rise to another "burst" as the inflammatory reaction evolves. In an effort to model such neuronal firing rate, we propose using an "integrate-and-fire" model which has been used for the impulse generation process of nerve cells (Sanderson 1980). This model transforms a continuoustime input signal into discrete-time series - a point process signal that in our case should be the neuronal spike train. A point process is a useful mathematical representation of a signal which consists of repeated similar events. The significance of the event is attached to the time at which it occurs rather than the detailed properties of the event itself. Prior studies (Chiu and Kao 2001; Olufsen, Tran et al. 2006) have employed the general principles of an integrate-and-fire model to characterize heart beats as discrete-time series in response to chemical concentrations (continuous signals).

In our model as described in Section 6.2, since the pro-inflammatory response (P) can signal to the autonomic nervous system via afferent sensory neurons (Elenkov 2008), we have assumed that $(\mathrm{P})$ response serves as the input signal to cardiac neurons. Thus, the integrate-and-fire model will transform the continuous-time $(\mathrm{P})$ signal into a train of 
spiking firing rates, $f_{\mathrm{p}}$. It is expected that a neuronal spike occurs every time the timedependent function, $f_{p}$, has deviated from its baseline (i.e. quantified by the value 1) and take the value 0 . When this event occurs the function, $f$, is reset to its baseline by the integrator component of the model (de Boer, Karemaker et al. 1985). Assuming that an increase in spiking firing rate is associated with increased release of neuromediator concentrations affecting heart rate, variations in heart rate as manifested by HRV can thereby be described by the time intervals where cardiac nerves have fired a pulse. Moreover, the general principles of the integrate-and-fire model can be applied to demonstrate the characteristics of beat-to-beat fluctuations in heart rate under simultaneous sympathetic and vagal controls as presented in Section 6.3. By considering the ANS influence (sympathetic/parasympathetic activities) as the input signal, the integrate-and-fire model will generate a heartbeat series and thus heart rate can be expressed as discrete-time series of interbeat intervals rather than a continuous-time form.

Further, in this modeling effort homeostasis can be viewed as a temporally invariant equilibrium steady state. However, physiologic and biochemical activities exhibit circadian rhythms(Green, Takahashi et al. 2008) and variations at both the level of autonomic and endocrine functions(Coogan and Wyse 2008; Lowry 2009). Thus, innate and adaptive immune activities are influenced by circadian rhythms and the diurnal incidence of some adverse events may be related to stress-induced alterations in the normal circadian pattern. Therefore, detailed knowledge about circadian aspects of the host defense system may be of considerable importance for the chronotherapy in neuroendocrine and autonomic disorders (Haus 2007). Modeling circadian aspects of the host defense system would provide the necessary ammunition for moving forward with the 
proposed integrated model predicting the influence of anti-inflammatory interventions in the context of physiological circadian therapy (Liu, Lewis et al. 2007).

Predicated upon the assumptions invoked in the development of the multiscale human inflammation model, an exogenously-induced prolonged stress condition, assessed by infusion kinetics (Hazra, Dubois et al. 2008; Ramakrishnan, DuBois et al. 2002a; Yao, Hoffman et al. 2008), will increase circulating levels of stress hormones followed by a concomitant increase in their pharmacodynamic effect. This effect will be manifested as potentiation in the anti-inflammatory signaling component (A) that gives rise to reduced levels of the pro-inflammatory response (P). Although the anti-inflammatory capacity of exogenously-induced acute hypercortisolemia or catecholamine excess is well established (Barber, Coyle et al. 1993; van der Poll, Coyle et al. 1996) such relationship varies considerably under prolonged stress condition(van der Poll, Barber et al. 1996; van der Poll, Coyle et al. 1996).Thus, the duration of exposure to stress (acute versus chronic) is an important distinguishing characteristic of stress-induced effects in the immune response altering the antecedent state of the in vivo host defense mechanisms. This dissertation is founded upon the hypothesis that during the early post-injury response the anti-inflammatory capacity of stress hormones is evident. However, alternative topologies may be explored (Porchet, Benowitz et al. 1988) with the availability of data related to the influence of extended period stress hormone infusion for simulating clinically severe stressful conditions.

It is important to realize that in silico models will never replace either biological or clinical research. They could, however, rationalize the decision making process by establishing the range of validity and predictability of intervention strategies. Finally, we 
would like to argue that possibly a very significant, and often overlooked, success of systems-based research is that through the universal language of mathematics and the opportunity of formalizing and quantifying abstract concepts of complex physiological phenomena, albeit with significant simplifications often times, it has managed to establish communication bridges between scientists from a variety of fields with a common goal: to develop a better understanding of a physiological condition. This could be one of the most significant impacts of systems-based translational research. 


\section{Bibliography}

Abreu, M. T., Arnold, E. T., Thomas, L. S., Gonsky, R., Zhou, Y., Hu, B., et al. (2002). "TLR4 and MD-2 expression is regulated by immune-mediated signals in human intestinal epithelial cells." J Biol Chem 277(23): 20431-7.

Aderem, A. and Smith, K. D. (2004). "A systems approach to dissecting immunity and inflammation." Semin Immunol 16(1): 55-67.

Aderem, A. (2005). "Systems biology: its practice and challenges." Cell 121(4): 511-3.

Alberti, C., Brun-Buisson, C., Chevret, S., Antonelli, M., Goodman, S. V., Martin, C., et al. (2005). "Systemic inflammatory response and progression to severe sepsis in critically ill infected patients." Am J Respir Crit Care Med 171(5): 461-8.

Aldridge, B. B., Burke, J. M., Lauffenburger, D. A. and Sorger, P. K. (2006). "Physicochemical modelling of cell signalling pathways." Nat Cell Biol 8(11): 1195-203.

Almon, R. R., DuBois, D. C., Brandenburg, E. H., Shi, W., Zhang, S., Straubinger, R. M., et al. (2002). "Pharmacodynamics and pharmacogenomics of diverse receptormediated effects of methylprednisolone in rats using microarray analysis." $\underline{\mathrm{J}}$ Pharmacokinet Pharmacodyn 29(2): 103-29.

Almon, R. R., Dubois, D. C., Jin, J. Y. and Jusko, W. J. (2005). "Pharmacogenomic responses of rat liver to methylprednisolone: an approach to mining a rich microarray time series." Aaps J 7(1): E156-94.

Almon, R. R., Lai, W., DuBois, D. C. and Jusko, W. J. (2005). "Corticosteroid-regulated genes in rat kidney: mining time series array data." Am J Physiol Endocrinol Metab 289(5): E870-82.

Almon, R. R., DuBois, D. C. and Jusko, W. J. (2007). "A microarray analysis of the temporal response of liver to methylprednisolone: a comparative analysis of two dosing regimens." Endocrinology 148(5): 2209-25.

Alvarez, S. M., Katsamanis Karavidas, M., Coyle, S. M., Lu, S. E., Macor, M., Oikawa, L. O., et al. (2007). "Low-dose steroid alters in vivo endotoxin-induced systemic inflammation but does not influence autonomic dysfunction." J Endotoxin Res 13(6): 358-68.

An, G. (2001). "Agent-based computer simulation and sirs: building a bridge between basic science and clinical trials." Shock 16(4): 266-73.

An, G. (2004). "In silico experiments of existing and hypothetical cytokine-directed clinical trials using agent-based modeling." Crit Care Med 32(10): 2050-60.

An, G. (2006). "Concepts for developing a collaborative in silico model of the acute inflammatory response using agent-based modeling." J Crit Care 21(1): 105-10; discussion 110-1.

An, G., Hunt, C. A., Clermont, G., Neugebauer, E. and Vodovotz, Y. (2007). "Challenges and rewards on the road to translational systems biology in acute illness: four case reports from interdisciplinary teams." J Crit Care 22(2): 169-75.

An, G. (2008). "Introduction of an agent-based multi-scale modular architecture for dynamic knowledge representation of acute inflammation." Theor Biol Med Model 5: 11. 
An, G., Faeder, J. and Vodovotz, Y. (2008). "Translational systems biology: introduction of an engineering approach to the pathophysiology of the burn patient." J Burn Care Res 29(2): 277-85.

Anderson, A. R. and Quaranta, V. (2008). "Integrative mathematical oncology." Nat Rev Cancer 8(3): 227-34.

Andreasen, A. S., Krabbe, K. S., Krogh-Madsen, R., Taudorf, S., Pedersen, B. K. and Moller, K. (2008). "Human endotoxemia as a model of systemic inflammation." Curr Med Chem 15(17): 1697-705.

Angus, D. C., Linde-Zwirble, W. T., Lidicker, J., Clermont, G., Carcillo, J. and Pinsky, M. R. (2001). "Epidemiology of severe sepsis in the United States: analysis of incidence, outcome, and associated costs of care." Crit Care Med 29(7): 1303-10.

Annane, D., Trabold, F., Sharshar, T., Jarrin, I., Blanc, A. S., Raphael, J. C., et al. (1999). "Inappropriate sympathetic activation at onset of septic shock: a spectral analysis approach." Am J Respir Crit Care Med 160(2): 458-65.

Annane, D., Sebille, V., Charpentier, C., Bollaert, P. E., Francois, B., Korach, J. M., et al. (2002). "Effect of treatment with low doses of hydrocortisone and fludrocortisone on mortality in patients with septic shock." Jama 288(7): 862-71.

Annane, D., Bellissant, E., Bollaert, P. E., Briegel, J., Keh, D. and Kupfer, Y. (2004a). "Corticosteroids for treating severe sepsis and septic shock." Cochrane Database Syst Rev(1): CD002243.

Annane, D., Bellissant, E., Bollaert, P. E., Briegel, J., Keh, D. and Kupfer, Y. (2004b). "Corticosteroids for severe sepsis and septic shock: a systematic review and metaanalysis." Bmj 329(7464): 480.

Annane, D., Bellissant, E. and Cavaillon, J. M. (2005). "Septic shock." Lancet 365(9453): 63-78.

Aronson, D., Mittleman, M. A. and Burger, A. J. (2001). "Interleukin-6 levels are inversely correlated with heart rate variability in patients with decompensated heart failure." J Cardiovasc Electrophysiol 12(3): 294-300.

Arzt, E., Sauer, J., Pollmacher, T., Labeur, M., Holsboer, F., Reul, J. M., et al. (1994). "Glucocorticoids suppress interleukin-1 receptor antagonist synthesis following induction by endotoxin." Endocrinology 134(2): 672-7.

Auphan, N., DiDonato, J. A., Rosette, C., Helmberg, A. and Karin, M. (1995). "Immunosuppression by glucocorticoids: inhibition of NF-kappa B activity through induction of I kappa B synthesis." Science 270(5234): 286-90.

Azimi, G. and Vincent, J. L. (1986). "Ultimate survival from septic shock." Resuscitation 14(4): 245-53.

Babu, M. M., Luscombe, N. M., Aravind, L., Gerstein, M. and Teichmann, S. A. (2004). "Structure and evolution of transcriptional regulatory networks." Curr Opin Struct Biol 14(3): 283-91.

Baldazzi, V., Castiglione, F. and Bernaschi, M. (2006). "An enhanced agent based model of the immune system response." Cell Immunol 244(2): 77-9.

Barabasi, A. L. and Oltvai, Z. N. (2004). "Network biology: understanding the cell's functional organization." Nat Rev Genet 5(2): 101-13.

Barber, A. E., Coyle, S. M., Marano, M. A., Fischer, E., Calvano, S. E., Fong, Y., et al. (1993). "Glucocorticoid therapy alters hormonal and cytokine responses to endotoxin in man." J Immunol 150(5): 1999-2006. 
Barnes, P. J. and Karin, M. (1997). "Nuclear factor-kappaB: a pivotal transcription factor in chronic inflammatory diseases." N Engl J Med 336(15): 1066-71.

Bauer, A. L., Beauchemin, C. A. A. and Perelson, A. S. (2009). "Agent-based modeling of host-pathogen systems: The successes and challenges." Information Sciences 179(10): 1379-1389.

Beard, D. A., Bassingthwaighte, J. B. and Greene, A. S. (2005). "Computational modeling of physiological systems." Physiol Genomics 23(1): 1-3; discussion 4.

Bendixen, H. H., Osgood, P. F., Hall, K. V. and Laver, M. B. (1964). "Dose-Dependent Differences in Catecholamine Action on Heart and Periphery." J Pharmacol Exp Ther 145: 299-306.

Bernard, G. R., Vincent, J. L., Laterre, P. F., LaRosa, S. P., Dhainaut, J. F., LopezRodriguez, A., et al. (2001). "Efficacy and safety of recombinant human activated protein C for severe sepsis." N Engl J Med 344(10): 699-709.

Berntson, G. G., Cacioppo, J. T. and Quigley, K. S. (1991). "Autonomic determinism: the modes of autonomic control, the doctrine of autonomic space, and the laws of autonomic constraint." Psychol Rev 98(4): 459-87.

Berntson, G. G., Bigger, J. T., Jr., Eckberg, D. L., Grossman, P., Kaufmann, P. G., Malik, M., et al. (1997). "Heart rate variability: origins, methods, and interpretive caveats." Psychophysiology 34(6): 623-48.

Beutler, B. and Rietschel, E. T. (2003). "Innate immune sensing and its roots: the story of endotoxin." Nat Rev Immunol 3(2): 169-76.

Bhattacharjee, R. N. and Akira, S. (2006). "Toll - like Receptor Signaling: Emerging Opportunities in Human Diseases and Medicine." Current Immunology Reviews 1: 81-90.

Blackwell, T. S. and Christman, J. W. (1996). "Sepsis and cytokines: current status." Br J Anaesth 77(1): 110-7.

Blalock, J. E. (2002). "Harnessing a neural-immune circuit to control inflammation and shock." J Exp Med 195(6): F25-8.

Bochud, P. Y. and Calandra, T. (2003). "Pathogenesis of sepsis: new concepts and implications for future treatment." Bmj 326(7383): 262-6.

Bone, R. C., Balk, R. A., Cerra, F. B., Dellinger, R. P., Fein, A. M., Knaus, W. A., et al. (1992). "Definitions for sepsis and organ failure and guidelines for the use of innovative therapies in sepsis. The ACCP/SCCM Consensus Conference Committee. American College of Chest Physicians/Society of Critical Care Medicine." Chest 101(6): 1644-55.

Bone, R. C., Grodzin, C. J. and Balk, R. A. (1997). "Sepsis: a new hypothesis for pathogenesis of the disease process." Chest 112(1): 235-43.

Bornstein, S. R. and Briegel, J. (2003). "A new role for glucocorticoids in septic shock: balancing the immune response." Am J Respir Crit Care Med 167(4): 485-6.

Borovikova, L. V., Ivanova, S., Zhang, M., Yang, H., Botchkina, G. I., Watkins, L. R., et al. (2000). "Vagus nerve stimulation attenuates the systemic inflammatory response to endotoxin." Nature 405(6785): 458-62.

Bosisio, D., Polentarutti, N., Sironi, M., Bernasconi, S., Miyake, K., Webb, G. R., et al. (2002). "Stimulation of toll-like receptor 4 expression in human mononuclear phagocytes by interferon-gamma: a molecular basis for priming and synergism with bacterial lipopolysaccharide." Blood 99(9): 3427-31. 
Bosshart, H. and Heinzelmann, M. (2007). "Targeting bacterial endotoxin: two sides of a coin." Ann N Y Acad Sci 1096: 1-17.

Brandtzaeg, P., Osnes, L., Ovstebo, R., Joo, G. B., Westvik, A. B. and Kierulf, P. (1996). "Net inflammatory capacity of human septic shock plasma evaluated by a monocyte-based target cell assay: identification of interleukin-10 as a major functional deactivator of human monocytes." J Exp Med 184(1): 51-60.

Brealey, D., Brand, M., Hargreaves, I., Heales, S., Land, J., Smolenski, R., et al. (2002). "Association between mitochondrial dysfunction and severity and outcome of septic shock." Lancet 360(9328): 219-23.

Briegel, J., Kellermann, W., Forst, H., Haller, M., Bittl, M., Hoffmann, G. E., et al. (1994). "Low-dose hydrocortisone infusion attenuates the systemic inflammatory response syndrome. The Phospholipase A2 Study Group." Clin Investig 72(10): 782-7.

Briegel, J., Jochum, M., Gippner-Steppert, C. and Thiel, M. (2001). "Immunomodulation in septic shock: hydrocortisone differentially regulates cytokine responses." J Am Soc Nephrol 12 Suppl 17: S70-4.

Brightbill, H. D., Plevy, S. E., Modlin, R. L. and Smale, S. T. (2000). "A prominent role for Sp1 during lipopolysaccharide-mediated induction of the IL-10 promoter in macrophages." J Immunol 164(4): 1940-51.

Brown, G. L. and Eccles, J. C. (1934). "The action of a single vagal volley on the rhythm of the heart beat." J Physiol 82(2): 211-41.

Bruce, J., Russell, E. M., Mollison, J. and Krukowski, Z. H. (2001). "The measurement and monitoring of surgical adverse events." Health Technol Assess 5(22): 1-194.

Calvano, S. E., Xiao, W., Richards, D. R., Felciano, R. M., Baker, H. V., Cho, R. J., et al. (2005a). "A network-based analysis of systemic inflammation in humans." Nature 437(7061): 1032-7.

Calvano, S. E., Xiao, W., Richards, D. R., Felciano, R. M., Baker, H. V., Cho, R. J., et al. (2005b). "A network-based analysis of systemic inflammation in humans." Nature.

Carmody, R. J. and Chen, Y. H. (2007). "Nuclear factor-kappaB: activation and regulation during toll-like receptor signaling." Cell Mol Immunol 4(1): 31-41.

Carre, J. E. and Singer, M. (2008). "Cellular energetic metabolism in sepsis: the need for a systems approach." Biochim Biophys Acta 1777(7-8): 763-71.

Cavaillon, J. M. and Annane, D. (2006). "Compartmentalization of the inflammatory response in sepsis and SIRS." J Endotoxin Res 12(3): 151-70.

Chan, P. L. and Holford, N. H. (2001). "Drug treatment effects on disease progression." Annu Rev Pharmacol Toxicol 41: 625-59.

Chiu, H. W. and Kao, T. (2001). "A mathematical model for autonomic control of heart rate variation." IEEE Eng Med Biol Mag 20(2): 69-76.

Chow, C. C., Clermont, G., Kumar, R., Lagoa, C., Tawadrous, Z., Gallo, D., et al. (2005). "The acute inflammatory response in diverse shock states." Shock 24(1): 74-84.

Chrousos, G. P. (1995). "The hypothalamic-pituitary-adrenal axis and immune-mediated inflammation." N Engl J Med 332(20): 1351-62.

Chung, T. P., Laramie, J. M., Meyer, D. J., Downey, T., Tam, L. H., Ding, H., et al. (2006). "Molecular diagnostics in sepsis: from bedside to bench." J Am Coll Surg 203(5): 585-598. 
Clermont, G., Bartels, J., Kumar, R., Constantine, G., Vodovotz, Y. and Chow, C. (2004). "In silico design of clinical trials: a method coming of age." Crit Care Med 32(10): 2061-70.

Cobb, J. P., Mindrinos, M. N., Miller-Graziano, C., Calvano, S. E., Baker, H. V., Xiao, W., et al. (2005). "Application of genome-wide expression analysis to human health and disease." Proc Natl Acad Sci U S A 102(13): 4801-6.

Contreras, M. and Ryan, L. M. (2000). "Fitting nonlinear and constrained generalized estimating equations with optimization software." Biometrics 56(4): 1268-71.

Coogan, A. N. and Wyse, C. A. (2008). "Neuroimmunology of the circadian clock." Brain Res 1232: 104-12.

Cook, D. N., Pisetsky, D. S. and Schwartz, D. A. (2004). "Toll-like receptors in the pathogenesis of human disease." Nat Immunol 5(10): 975-9.

Cook, J. A. (1998). "Molecular basis of endotoxin tolerance." Ann N Y Acad Sci 851: 426-8.

Cook, S., Togni, M., Schaub, M. C., Wenaweser, P. and Hess, O. M. (2006). "High heart rate: a cardiovascular risk factor?" Eur Heart J 27(20): 2387-93.

Copeland, S., Warren, H. S., Lowry, S. F., Calvano, S. E. and Remick, D. (2005). "Acute inflammatory response to endotoxin in mice and humans." Clin Diagn Lab Immunol 12(1): 60-7.

Costa, M., Goldberger, A. L. and Peng, C. K. (2002). "Multiscale entropy analysis of complex physiologic time series." Phys Rev Lett 89(6): 068102.

Court, O., Kumar, A., Parrillo, J. E. and Kumar, A. (2002). "Clinical review: Myocardial depression in sepsis and septic shock." Crit Care 6(6): 500-8.

Cross, A. S. and Opal, S. M. (2003). "A new paradigm for the treatment of sepsis: is it time to consider combination therapy?" Ann Intern Med 138(6): 502-5.

Czura, C. J. and Tracey, K. J. (2005). "Autonomic neural regulation of immunity." Intern Med 257(2): 156-66.

Davos, C. H., Davies, L. C. and Piepoli, M. (2002). "The effect of baroreceptor activity on cardiovascular regulation." Hellenic J Cardiol 43: 143-155.

Day, J., Rubin, J., Vodovotz, Y., Chow, C. C., Reynolds, A. and Clermont, G. (2006). "A reduced mathematical model of the acute inflammatory response II. Capturing scenarios of repeated endotoxin administration." J Theor Biol 242(1): 237-56.

de Boer, R. W., Karemaker, J. M. and Strackee, J. (1985). "Spectrum of a series of point events, generated by the integral pulse frequency modulation model." Med Biol Eng Comput 23(2): 138-42.

Decker, T. (2004). "Sepsis: avoiding its deadly toll." J Clin Invest 113(10): 1387-9.

Delhase, M., Hayakawa, M., Chen, Y. and Karin, M. (1999). "Positive and Negative Regulation of IkB Kinase Activity Through IKKb Subunit Phosphorylation." Science 284: 309-13.

Dellinger, R. P., Levy, M. M., Carlet, J. M., Bion, J., Parker, M. M., Jaeschke, R., et al. (2008). "Surviving Sepsis Campaign: international guidelines for management of severe sepsis and septic shock: 2008." Crit Care Med 36(1): 296-327.

Derendorf, H., Lesko, L. J., Chaikin, P., Colburn, W. A., Lee, P., Miller, R., et al. (2000). "Pharmacokinetic/pharmacodynamic modeling in drug research and development." J Clin Pharmacol 40(12 Pt 2): 1399-418. 
Desborough, J. P. (2000). "The stress response to trauma and surgery." Br J Anaesth 85(1): 109-17.

Detilleux, J., Vangroenweghe, F. and Burvenich, C. (2006). "Mathematical model of the acute inflammatory response to Escherichia coli in intramammary challenge." $\mathrm{J}$ Dairy Sci 89(9): 3455-65.

Du, X., Poltorak, A., Silva, M. and Beutler, B. (1999). "Analysis of Tlr4-mediated LPS signal transduction in macrophages by mutational modification of the receptor." Blood Cells Mol Dis 25(5-6): 328-38.

DuBois, D. C., Xu, Z. X., McKay, L., Almon, R. R., Pyszcznski, N. and Jusko, W. J. (1995). "Differential dynamics of receptor down-regulation and tyrosine aminotransferase induction following glucocorticoid treatment." $\underline{\mathrm{J} \text { Steroid }}$ Biochem Mol Biol 54(5-6): 237-43.

Dunser, M. W. and Hasibeder, W. R. (2006). "Vasopressin in vasodilatory shock: ensure organ blood flow, but take care of the heart!" Crit Care 10(6): 172.

Dunser, M. W. and Hasibeder, W. R. (2009). "Sympathetic overstimulation during critical illness: adverse effects of adrenergic stress." J Intensive Care Med 24(5): 293-316.

Elenkov, I. J., Wilder, R. L., Chrousos, G. P. and Vizi, E. S. (2000). "The sympathetic nerve--an integrative interface between two supersystems: the brain and the immune system." Pharmacol Rev 52(4): 595-638.

Elenkov, I. J. (2008). "Neurohormonal-cytokine interactions: implications for inflammation, common human diseases and well-being." Neurochem Int 52(1-2): 40-51.

Ermentrout, G. B. and Edelstein-Keshet, L. (1993). "Cellular automata approaches to biological modeling." J Theor Biol 160(1): 97-133.

Fan, H. and Cook, J. A. (2004). "Molecular mechanisms of endotoxin tolerance." Endotoxin Res 10(2): 71-84.

Fannin, R. D., Auman, J. T., Bruno, M. E., Sieber, S. O., Ward, S. M., Tucker, C. J., et al. (2005). "Differential gene expression profiling in whole blood during acute systemic inflammation in lipopolysaccharide-treated rats." $\underline{\text { Physiol Genomics }}$ 21(1): 92-104.

Fisher, E. S. and Lauffenburger, D. A. (1990). "Analysis of the effects of immune cell motility and chemotaxis on target elimination dynamics." Math Biosci 98(1): 73102.

Fitting, C., Dhawan, S. and Cavaillon, J. M. (2004). "Compartmentalization of tolerance to endotoxin." J Infect Dis 189(7): 1295-303.

Folcik, V. A., An, G. C. and Orosz, C. G. (2007). "The Basic Immune Simulator: an agent-based model to study the interactions between innate and adaptive immunity." Theor Biol Med Model 4: 39.

Fong, Y. M., Marano, M. A., Moldawer, L. L., Wei, H., Calvano, S. E., Kenney, J. S., et al. (1990). "The acute splanchnic and peripheral tissue metabolic response to endotoxin in humans." J Clin Invest 85(6): 1896-904.

Foteinou, P. T., Calvano, S. E., Lowry, S. F. and Androulakis, I. P. (2007). "An indirect response model of endotoxin-induced systemic inflammation." Journal of Critical Care 22(4): 337-338. 
Foteinou, P. T., Calvano, S. E., Lowry, S. F. and Androulakis, I. P. (2009a). "Modeling endotoxin-induced systemic inflammation using an indirect response approach." Math Biosci 217(1): 27-42.

Foteinou, P. T., Calvano, S. E., Lowry, S. F. and Androulakis, I. P. (2009b). "In silico simulation of corticosteroids effect on an NFkB- dependent physicochemical model of systemic inflammation." PLoS One 4(3): e4706.

Foteinou, P. T., Calvano, S. E., Lowry, S. F. and Androulakis, I. P. (2009c). "Translational Potential of Systems-Based Models of Inflammation." Clinical and Translational Science 2(1): 85-89.

Foteinou, P. T., Calvano, S. E., Lowry, S. F. and Androulakis, I. P. (2010-in press). "A Multi-scale Model for the Assessment of Autonomic Dysfunction in Human Endotoxemia." Phys Genomics.

Funk, G. A., Jansen, V. A., Bonhoeffer, S. and Killingback, T. (2005). "Spatial models of virus-immune dynamics." J Theor Biol 233(2): 221-36.

Gelijns, A. C., Rosenberg, N. and Moskowitz, A. J. (1998). "Capturing the unexpected benefits of medical research." N Engl J Med 339(10): 693-8.

Glick, G. and Braunwald, E. (1965). "Relative Roles of the Sympathetic and Parasympathetic Nervous Systems in the Reflex Control of Heart Rate." Circ Res 16: 363-75.

Godin, P. J. and Buchman, T. G. (1996). "Uncoupling of biological oscillators: a complementary hypothesis concerning the pathogenesis of multiple organ dysfunction syndrome." Crit Care Med 24(7): 1107-16.

Godin, P. J., Fleisher, L. A., Eidsath, A., Vandivier, R. W., Preas, H. L., Banks, S. M., et al. (1996). "Experimental human endotoxemia increases cardiac regularity: results from a prospective, randomized, crossover trial." Crit Care Med 24(7): 1117-24.

Goldberger, J. J., Challapalli, S., Tung, R., Parker, M. A. and Kadish, A. H. (2001). "Relationship of heart rate variability to parasympathetic effect." Circulation 103(15): 1977-83.

Goldstein, D. S. (1981). "Plasma norepinephrine as an indicator of sympathetic neural activity in clinical cardiology." Am J Cardiol 48(6): 1147-54.

Goldstein, D. S., McCarty, R., Polinsky, R. J. and Kopin, I. J. (1983). "Relationship between plasma norepinephrine and sympathetic neural activity." Hypertension 5(4): 552-9.

Goldstein, D. S., Eisenhofer, G. and Kopin, I. J. (2003). "Sources and significance of plasma levels of catechols and their metabolites in humans." J Pharmacol Exp Ther 305(3): 800-11.

Grassi, G. and Esler, M. (1999). "How to assess sympathetic activity in humans." 즌 Hypertens 17(6): 719-34.

Green, C. B., Takahashi, J. S. and Bass, J. (2008). "The meter of metabolism." Cell 134(5): 728-42.

Greisman, S. E., Hornick, R. B., Wagner, H. N., Jr., Woodward, W. E. and Woodward, T. E. (1969). "The role of endotoxin during typhoid fever and tularemia in man. IV. The integrity of the endotoxin tolerance mechanisms during infection." $\mathrm{J}$ Clin Invest 48(4): 613-29.

Gri, G., Savio, D., Trinchieri, G. and Ma, X. (1998). "Synergistic regulation of the human interleukin-12 p40 promoter by NFkappaB and Ets transcription factors in 
Epstein-Barr virus-transformed B cells and macrophages." J Biol Chem 273(11): 6431-8.

Guha, M. and Mackman, N. (2001). "LPS induction of gene expression in human monocytes." Cell Signal 13(2): 85-94.

Gupta, S. and Gollapudi, S. (2005). "Molecular mechanisms of TNF-alpha-induced apoptosis in aging human T cell subsets." Int J Biochem Cell Biol 37(5): 1034-42.

Gutkin, B. S., Dehaene, S. and Changeux, J. P. (2006). "A neurocomputational hypothesis for nicotine addiction." Proc Natl Acad Sci U S A 103(4): 1106-11.

Hall, J. E. (2001). "The promise of translational physiology." Am J Physiol Cell Physiol 281(5): C1411-2.

Hancioglu, B., Swigon, D. and Clermont, G. (2007). "A dynamical model of human immune response to influenza A virus infection." J Theor Biol 246(1): 70-86.

Hasko, G., Elenkov, I. J., Kvetan, V. and Vizi, E. S. (1995). "Differential effect of selective block of alpha 2-adrenoreceptors on plasma levels of tumour necrosis factor-alpha, interleukin- 6 and corticosterone induced by bacterial lipopolysaccharide in mice." J Endocrinol 144(3): 457-62.

Haus, E. (2007). "Chronobiology in the endocrine system." Adv Drug Deliv Rev 59(910): 985-1014.

Hawes, A. S., Rock, C. S., Keogh, C. V., Lowry, S. F. and Calvano, S. E. (1992). "In vivo effects of the antiglucocorticoid RU 486 on glucocorticoid and cytokine responses to Escherichia coli endotoxin." Infect Immun 60(7): 2641-7.

Hazra, A., Dubois, D. C., Almon, R. R., Snyder, G. H. and Jusko, W. J. (2008). "Pharmacodynamic modeling of acute and chronic effects of methylprednisolone on hepatic urea cycle genes in rats." Gene Regul Syst Bio 2: 1-19.

Hoffmann, A., Levchenko, A., Scott, M. and Baltimore, D. (2002a). "The IB-NF-B Signaling Module: Temporal Control and Selective Gene Activation." Science 298(5596): 1241-45.

Hoffmann, A., Levchenko, A., Scott, M. L. and Baltimore, D. (2002b). "The IkappaBNF-kappaB signaling module: temporal control and selective gene activation." Science 298(5596): 1241-5.

Hotchkiss, R. S. and Karl, I. E. (2003). "The pathophysiology and treatment of sepsis." N Engl J Med 348(2): 138-50.

Houle, M. S. and Billman, G. E. (1999). "Low-frequency component of the heart rate variability spectrum: a poor marker of sympathetic activity." Am J Physiol 276(1 Pt 2): H215-23.

Huang, A. C., Hu, L., Kauffman, S. A., Zhang, W. and Shmulevich, I. (2009). "Using cell fate attractors to uncover transcriptional regulation of HL60 neutrophil differentiation." BMC Syst Biol 3: 20.

Huang, S., Eichler, G., Bar-Yam, Y. and Ingber, D. E. (2005). "Cell fates as highdimensional attractor states of a complex gene regulatory network." Phys Rev Lett 94(12): 128701.

Huikuri, H. V., Makikallio, T., Airaksinen, K. E., Mitrani, R., Castellanos, A. and Myerburg, R. J. (1999). "Measurement of heart rate variability: a clinical tool or a research toy?" J Am Coll Cardiol 34(7): 1878-83. 
Ignatowski, T. A. and Spengler, R. N. (1995). "Regulation of macrophage-derived tumor necrosis factor production by modification of adrenergic receptor sensitivity." $\underline{\mathrm{J}}$ Neuroimmunol 61(1): 61-70.

Ihekwaba, A. E., Broomhead, D. S., Grimley, R. L., Benson, N. and Kell, D. B. (2004). "Sensitivity analysis of parameters controlling oscillatory signalling in the NFkappaB pathway: the roles of IKK and IkappaBalpha." Syst Biol (Stevenage) 1(1): 93-103.

Jan, B. U., Coyle, S. M., Oikawa, L. O., Lu, S. E., Calvano, S. E., Lehrer, P. M., et al. (2009). "Influence of acute epinephrine infusion on endotoxin-induced parameters of heart rate variability: a randomized controlled trial." Ann Surg 249(5): 750-6.

Jin, J. Y., Almon, R. R., DuBois, D. C. and Jusko, W. J. (2003). "Modeling of corticosteroid pharmacogenomics in rat liver using gene microarrays." $\underline{J}$ Pharmacol Exp Ther 307(1): 93-109.

Josa, M., Urizar, J. P., Rapado, J., Dios-Vieitez, C., Castaneda-Hernandez, G., FloresMurrieta, F., et al. (2001). "Pharmacokinetic/pharmacodynamic modeling of antipyretic and anti-inflammatory effects of naproxen in the rat." J Pharmacol Exp Ther 297(1): 198-205.

Jusko, W. J. (1994). "Receptor-mediated pharmacodynamics of corticosteroids." Prog Clin Biol Res 387: 261-70.

Jusko, W. J. and Ko, H. C. (1994). "Physiologic indirect response models characterize diverse types of pharmacodynamic effects." Clin Pharmacol Ther 56(4): 406-19.

Jusko, W. J., DuBois, D. and Almon, R. (2005). "Sixth-Generation Model for Corticosteroid Pharmacodynamics: Multi-Hormonal Regulation of Tyrosine Aminotransferase in Rat Liver." J Pharmacokin Pharmacodyn.

Karin, M. and Delhase, M. (2000). "The I kappa B kinase (IKK) and NF-kappa B: key elements of proinflammatory signalling." Semin Immunol 12(1): 85-98.

Kataoka, M., Ito, C., Sasaki, H., Yamane, K. and Kohno, N. (2004). "Low heart rate variability is a risk factor for sudden cardiac death in type 2 diabetes." Diabetes Res Clin Pract 64(1): 51-8.

Keh, D., Boehnke, T., Weber-Cartens, S., Schulz, C., Ahlers, O., Bercker, S., et al. (2003). "Immunologic and hemodynamic effects of "low-dose" hydrocortisone in septic shock: a double-blind, randomized, placebo-controlled, crossover study." Am J Respir Crit Care Med 167(4): 512-20.

Kerschen, E. J., Fernandez, J. A., Cooley, B. C., Yang, X. V., Sood, R., Mosnier, L. O., et al. (2007). "Endotoxemia and sepsis mortality reduction by non-anticoagulant activated protein C." J Exp Med 204(10): 2439-48.

Kidd, P. (2003). "Th1/Th2 balance: the hypothesis, its limitations, and implications for health and disease." Altern Med Rev 8(3): 223-46.

Kiehl, T. R., Mattheyses, R. M. and Simmons, M. K. (2004). "Hybrid simulation of cellular behavior." Bioinformatics 20(3): 316-22.

Kirkpatrick S., C. D. G., Jr., P. Vecchi (1983). "Optimization by simulated annealing." Science 220.

Kishore, R., McMullen, M. R., Cocuzzi, E. and Nagy, L. E. (2004). "Lipopolysaccharidemediated signal transduction: Stabilization of TNF-alpha mRNA contributes to increased lipopolysaccharide-stimulated TNF-alpha production by Kupffer cells after chronic ethanol feeding." Comp Hepatol 3 Suppl 1: S31. 
Kitano, H. (2002). "Systems biology: a brief overview." Science 295(5560): 1662-4.

Klaitman, V. and Almog, Y. (2003). "Corticosteroids in sepsis: a new concept for an old drug." Isr Med Assoc J 5(1): 51-5.

Klein, M. B., Silver, G., Gamelli, R. L., Gibran, N. S., Herndon, D. N., Hunt, J. L., et al. (2006). "Inflammation and the host response to injury: an overview of the multicenter study of the genomic and proteomic response to burn injury." J Burn Care Res 27(4): 448-51.

Klinke, D. J., Ustyugova, I. V., Brundage, K. M. and J.B., B. (2008). "Modulating Temporal Control of NF-kappaB Activation: Implications for Therapeutic and Assay Selection (un-edited manuscript) " Biophys J BioFAST.

Kochanek, K. D. and Smith, B. L. (2004). "Deaths: preliminary data for 2002." Natl Vital Stat Rep 52(13): 1-47.

Krishna, S., Jensen, M. H. and Sneppen, K. (2006). "Minimal model of spiky oscillations in NF-kappaB signaling." Proc Natl Acad Sci U S A 103(29): 10840-5.

Krishnamoorthy, S. and Honn, K. V. (2006). "Inflammation and disease progression." Cancer Metastasis Rev 25(3): 481-91.

Krzyzanski, W. and Jusko, W. J. (1997). "Mathematical formalism for the properties of four basic models of indirect pharmacodynamic responses." $\underline{\mathrm{J} \text { Pharmacokinet }}$ Biopharm 25(1): 107-23.

Kumar, R., Clermont, G., Vodovotz, Y. and Chow, C. C. (2004). "The dynamics of acute inflammation." J Theor Biol 230(2): 145-55.

Kumar, R., Chow, C. C., Bartels, J. D., Clermont, G. and Vodovotz, Y. (2008). "A mathematical simulation of the inflammatory response to anthrax infection." Shock 29(1): 104-11.

Lagoa, C. E., Bartels, J., Baratt, A., Tseng, G., Clermont, G., Fink, M. P., et al. (2006). "The role of initial trauma in the host's response to injury and hemorrhage: insights from a correlation of mathematical simulations and hepatic transcriptomic analysis." Shock 26(6): 592-600.

Lameris, T. W., de Zeeuw, S., Duncker, D. J., Tietge, W., Alberts, G., Boomsma, F., et al. (2002). "Epinephrine in the heart: uptake and release, but no facilitation of norepinephrine release." Circulation 106(7): 860-5.

Lampariello, F. (2000). "On the use of the Kolmogorov-Smirnov statistical test for immunofluorescence histogram comparison." Cytometry 39(3): 179-88.

Laroux, F. S. (2004). "Mechanisms of inflammation: the good, the bad and the ugly." Front Biosci 9: 3156-62.

Lauffenburger, D. A. and Kennedy, C. R. (1980). "Analysis of a Lumped Model for Tissue Inflammation Dynamics." Mathematical Biosciences 53: 189-221.

Lauffenburger, D. A. and Linderman, J. J. (1996). "Receptors. Models for Binding, Trafficking, and Signalling." The International Journal of Biochemistry and Cell Biology 28: 1418-1418.

Lefering, R. and Neugebauer, E. A. (1995). "Steroid controversy in sepsis and septic shock: a meta-analysis." Crit Care Med 23(7): 1294-303.

Lehmann, V., Freudenberg, M. A. and Galanos, C. (1987). "Lethal toxicity of lipopolysaccharide and tumor necrosis factor in normal and D-galactosaminetreated mice." J Exp Med 165(3): 657-63. 
Levy, R. L., White, P. D., Stroud, W. D. and Hillman, C. C. (1945). "Transient tachycardia: prognostic significance alone in association with transient hypertension." JAMA 129: 585-588.

Li, Q. and Verma, I. M. (2002). "NF-kappaB regulation in the immune system." Nat Rev Immunol 2(10): 725-34.

Ligtenberg, J. J., Girbes, A. R., Beentjes, J. A., Tulleken, J. E., van der Werf, T. S. and Zijlstra, J. G. (2001). "Hormones in the critically ill patient: to intervene or not to intervene?" Intensive Care Med 27(10): 1567-77.

Lin, E. and Lowry, S. F. (1998). "The Human Response to Endotoxin " Sepsis 2: 255262.

Lin, E., Calvano, S. E., Coyle, S. M. and Lowry, S. F. (1999). "Physiologic hypercortisolemia in humasna modulates CD95-signal transduction." Surg Forum 50: $288-290$.

Liu, A. C., Lewis, W. G. and Kay, S. A. (2007). "Mammalian circadian signaling networks and therapeutic targets." Nat Chem Biol 3(10): 630-9.

Louzoun, Y., Solomon, S., Atlan, H. and Cohen, I. R. (2001). "Modeling complexity in biology." Physica A 297(1-2): 242-252.

Lowry, S. F. (2005). "Human endotoxemia: a model for mechanistic insight and therapeutic targeting." Shock 24 Suppl 1: 94-100.

Lowry, S. F. and Calvano, S. E. (2008). "Challenges for modeling and interpreting the complex biology of severe injury and inflammation." J Leukoc Biol 83(3): 553-7.

Lowry, S. F. (2009). "The stressed host response to infection: the disruptive signals and rhythms of systemic inflammation." Surg Clin North Am 89(2): 311-26.

Mackay, C. R. (2000). "Follicular homing T helper (Th) cells and the Th1/Th2 paradigm." J Exp Med 192(11): F31-4.

MacMaster, J. F., Dambach, D. M., Lee, D. B., Berry, K. K., Qiu, Y., Zusi, F. C., et al. (2003). "An inhibitor of IkappaB kinase, BMS-345541, blocks endothelial cell adhesion molecule expression and reduces the severity of dextran sulfate sodiuminduced colitis in mice." Inflamm Res 52(12): 508-11.

Mager, D. E. and Jusko, W. J. (2001). "Pharmacodynamic modeling of time-dependent transduction systems." Clin Pharmacol Ther 70(3): 210-6.

Mager, D. E., Lin, S. X., Blum, R. A., Lates, C. D. and Jusko, W. J. (2003). "Dose equivalency evaluation of major corticosteroids: pharmacokinetics and cell trafficking and cortisol dynamics." J Clin Pharmacol 43(11): 1216-27.

Mager, D. E., Wyska, E. and Jusko, W. J. (2003). "Diversity of mechanism-based pharmacodynamic models." Drug Metab Dispos 31(5): 510-8.

Mahony, D. S. O., Pham, U., Iyer, R., Hawn, T. R. and Liles, W. C. (2008). "Differential constitutive and Cytokine-Modulated Expression of Human Toll-like Receptors in Primary Neutrophils, Monocytes, and Macrophages." International Journal of Medical Sciences 5(1): 1-8.

Malave, H. A., Taylor, A. A., Nattama, J., Deswal, A. and Mann, D. L. (2003). "Circulating levels of tumor necrosis factor correlate with indexes of depressed heart rate variability: a study in patients with mild-to-moderate heart failure." Chest 123(3): 716-24. 
Marshall, J. C., Walker, P. M., Foster, D. M., Harris, D., Ribeiro, M., Paice, J., et al. (2002). "Measurement of endotoxin activity in critically ill patients using whole blood neutrophil dependent chemiluminescence." Crit Care 6(4): 342-8.

Marshall, J. C. (2003). "Such stuff as dreams are made on: mediator-directed therapy in sepsis." Nat Rev Drug Discov 2(5): 391-405.

Marshall, J. C. (2005). "Modeling MODS: what can be learned from animal models of the multiple-organ dysfunction syndrome?" Intensive Care Med 31(5): 605-8.

Marshall, J. C., Deitch, E., Moldawer, L. L., Opal, S., Redl, H. and van der Poll, T. (2005). "Preclinical models of shock and sepsis: what can they tell us?" Shock 24 Suppl 1: 1-6.

Marsland, A. L., Gianaros, P. J., Prather, A. A., Jennings, J. R., Neumann, S. A. and Manuck, S. B. (2007). "Stimulated production of proinflammatory cytokines covaries inversely with heart rate variability." Psychosom Med 69(8): 709-16.

Martich, G. D., Danner, R. L., Ceska, M. and Suffredini, A. F. (1991). "Detection of interleukin 8 and tumor necrosis factor in normal humans after intravenous endotoxin: the effect of antiinflammatory agents." J Exp Med 173(4): 1021-4.

Matsuda, N. and Hattori, Y. (2006). "Systemic inflammatory response syndrome (SIRS): molecular pathophysiology and gene therapy." J Pharmacol Sci 101(3): 189-98.

Matthay, M. A. and Ware, L. B. (2004). "Can nicotine treat sepsis?" Nat Med 10(11): 1161-2.

Mayer, H., Zaenker, K. S. and An Der Heiden, U. (1995). "A basic mathematical model of the immune response." Chaos 5(1): 155-161.

McCall, C. E., Grosso-Wilmoth, L. M., LaRue, K., Guzman, R. N. and Cousart, S. L. (1993). "Tolerance to endotoxin-induced expression of the interleukin-1 beta gene in blood neutrophils of humans with the sepsis syndrome." J Clin Invest 91(3): 853-61.

McKnight, A. J., Zimmer, G. J., Fogelman, I., Wolf, S. F. and Abbas, A. K. (1994). "Effects of IL-12 on helper T cell-dependent immune responses in vivo." $\underline{\mathrm{J}}$ Immunol 152(5): 2172-9.

Meduri, G. U., Headley, A. S., Golden, E., Carson, S. J., Umberger, R. A., Kelso, T., et al. (1998). "Effect of prolonged methylprednisolone therapy in unresolving acute respiratory distress syndrome: a randomized controlled trial." Jama 280(2): 15965.

Mi, Q., Riviere, B., Clermont, G., Steed, D. L. and Vodovotz, Y. (2007). "Agent-based model of inflammation and wound healing: insights into diabetic foot ulcer pathology and the role of transforming growth factor-beta1." Wound Repair Regen 15(5): 671-82.

Michie, H. R., Manogue, K. R., Spriggs, D. R., Revhaug, A., O'Dwyer, S., Dinarello, C. A., et al. (1988). "Detection of circulating tumor necrosis factor after endotoxin administration." N Engl J Med 318(23): 1481-6.

Miller, R., Ewy, W., Corrigan, B. W., Ouellet, D., Hermann, D., Kowalski, K. G., et al. (2005). "How modeling and simulation have enhanced decision making in new drug development." J Pharmacokinet Pharmacodyn 32(2): 185-97.

Mittal, R., Peak-Chew, S. Y. and McMahon, H. T. (2006). "Acetylation of MEK2 and I kappa B kinase (IKK) activation loop residues by YopJ inhibits signaling." Proc Natl Acad Sci U S A 103(49): 18574-9. 
Monick, M. M. and Hunninghake, G. W. (2002). "Activation of second messenger pathways in alveolar macrophages by endotoxin." Eur Respir J 20(1): 210-22.

Morris, J. A., Jr., Norris, P. R., Ozdas, A., Waitman, L. R., Harrell, F. E., Jr., Williams, A. E., et al. (2006). "Reduced heart rate variability: an indicator of cardiac uncoupling and diminished physiologic reserve in 1,425 trauma patients." $\underline{\mathrm{J}}$ Trauma 60(6): 1165-73; discussion 1173-4.

Morris, J. A., Jr., Norris, P. R., Waitman, L. R., Ozdas, A., Guillamondegui, O. D. and Jenkins, J. M. (2007). "Adrenal insufficiency, heart rate variability, and complex biologic systems: a study of 1,871 critically ill trauma patients." J Am Coll Surg 204(5): 885-92; discussion 892-3.

Mueller, H. S. and Ayres, S. M. (1980). "Propranolol decreases sympathetic nervous activity reflected by plasma catecholamines during evolution of myocardial infarction in man." J Clin Invest 65(2): 338-46.

Munford, R. S. (2006). "Severe Sepsis and Septic Shock: The Role of Gram - Negative Bacteremia." Annu.Rev.Pathol.Mech.Dis. 1: 467-496.

Munoz, C., Carlet, J., Fitting, C., Misset, B., Bleriot, J. P. and Cavaillon, J. M. (1991). "Dysregulation of in vitro cytokine production by monocytes during sepsis." J Clin Invest 88(5): 1747-54.

Murai, T., Nakagawa, Y. and Ogawa, Y. (1996). "Potentiation of lethal endotoxin shock by streptococcal pyrogenic exotoxin in rabbits: possible relevance of hyperreactivity of macrophages to endotoxin." FEMS Immunol Med Microbiol 13(4): 269-72.

Murray, P. J. (2007). "The JAK-STAT signaling pathway: input and output integration." J Immunol 178(5): 2623-9.

Neugebauer, E. A., Willy, C. and Sauerland, S. (2001). "Complexity and non-linearity in shock research: reductionism or synthesis?" Shock 16(4): 252-8.

Nomura, F., Akashi, S., Sakao, Y., Sato, S., Kawai, T., Matsumoto, M., et al. (2000). "Cutting edge: endotoxin tolerance in mouse peritoneal macrophages correlates with down-regulation of surface toll-like receptor 4 expression." J Immunol 164(7): 3476-9.

Norris, P. R., Morris, J. A., Jr., Ozdas, A., Grogan, E. L. and Williams, A. E. (2005). "Heart rate variability predicts trauma patient outcome as early as $12 \mathrm{~h}$ : implications for military and civilian triage." J Surg Res 129(1): 122-8.

Norris, P. R., Ozdas, A., Cao, H., Williams, A. E., Harrell, F. E., Jenkins, J. M., et al. (2006). "Cardiac uncoupling and heart rate variability stratify ICU patients by mortality: a study of 2088 trauma patients." Ann Surg 243(6): 804-12; discussion 812-4.

Norris, P. R., Stein, P. K. and Morris, J. A., Jr. (2008). "Reduced heart rate multiscale entropy predicts death in critical illness: a study of physiologic complexity in 285 trauma patients." J Crit Care 23(3): 399-405.

Nystrom, P. O. (1998). "The systemic inflammatory response syndrome: definitions and aetiology." J Antimicrob Chemother 41 Suppl A: 1-7.

Olufsen, M. S., Tran, H. T., Ottesen, J. T., Lipsitz, L. A., Novak, V., Program, R. E. U., et al. (2006). "Modeling baroreflex regulation of heart rate during orthostatic stress." American Journal of Physiology-Regulatory Integrative and Comparative Physiology 291(5): R1355-R1368. 
Opal, S. M. and DePalo, V. A. (2000). "Anti-inflammatory cytokines." Chest 117(4): 1162-72.

Padgett, D. A. and Glaser, R. (2003). "How stress influences the immune response." Trends Immunol 24(8): 444-8.

Palatini, P. and Julius, S. (1997). "Heart rate and the cardiovascular risk." J Hypertens 15(1): 3-17.

Palatini, P. and Julius, S. (2004). "Elevated heart rate: a major risk factor for cardiovascular disease." Clin Exp Hypertens 26(7-8): 637-44.

Palatini, P. (2007). "Heart rate as an independent risk factor for cardiovascular disease: current evidence and basic mechanisms." Drugs 67 Suppl 2: 3-13.

Palatini, P. (2009). "Elevated heart rate: a "new" cardiovascular risk factor?" Prog Cardiovase Dis 52(1): 1-5.

Parker, M. M., Shelhamer, J. H., Natanson, C., Alling, D. W. and Parrillo, J. E. (1987). "Serial cardiovascular variables in survivors and nonsurvivors of human septic shock: heart rate as an early predictor of prognosis." Crit Care Med 15(10): 923-9.

Pavlov, V. A. and Tracey, K. J. (2004). "Neural regulators of innate immune responses and inflammation." Cell Mol Life Sci 61(18): 2322-31.

Perlstein, I., Stepensky, D., Sapoznikov, D. and Hoffman, A. (2001). "Power spectral analysis of heart rate variability in rats as a quantitative tool in the PK-PD analysis of the parasympatholytic activity of atropine." Pharm Res 18(8): 1220-5.

Perlstein, I., Stepensky, D., Krzyzanski, W. and Hoffman, A. (2002). "A signal transduction pharmacodynamic model of the kinetics of the parasympathomimetic activity of low-dose scopolamine and atropine in rats." J Pharm Sci 91(12): 250010.

Pogson, M., Holcombe, M., Smallwood, R. and Qwarnstrom, E. (2008). "Introducing spatial information into predictive NF-kappaB modelling--an agent-based approach." PLoS ONE 3(6): e2367.

Poll, v. d. (1996). J Infect Disease 174: 1356-1360.

Porchet, H. C., Benowitz, N. L. and Sheiner, L. B. (1988). "Pharmacodynamic model of tolerance: application to nicotine." J Pharmacol Exp Ther 244(1): 231-6.

Post, T. M., Freijer, J. I., DeJongh, J. and Danhof, M. (2005). "Disease system analysis: basic disease progression models in degenerative disease." Pharm Res 22(7): 1038-49.

Prabhakar, U., Conway, T. M., Murdock, P., Mooney, J. L., Clark, S., Hedge, P., et al. (2005). "Correlation of protein and gene expression profiles of inflammatory proteins after endotoxin challenge in human subjects." DNA Cell Biol 24(7): 41031.

Prince, J. M., Levy, R. M., Bartels, J., Baratt, A., Kane, J. M., 3rd, Lagoa, C., et al. (2006). "In silico and in vivo approach to elucidate the inflammatory complexity of CD14-deficient mice." Mol Med 12(4-6): 88-96.

Protti, A. and Singer, M. (2007). "Strategies to modulate cellular energetic metabolism during sepsis." Novartis Found Symp 280: 7-16; discussion 16-20, 160-4.

Rajasethupathy, P., Vayttaden, S. J. and Bhalla, U. S. (2005). "Systems modeling: a pathway to drug discovery." Curr Opin Chem Biol 9(4): 400-6.

Ramakrishnan, N. and Bhalla, U. S. (2008). "Memory switches in chemical reaction space." PLoS Comput Biol 4(7): e1000122. 
Ramakrishnan, R., DuBois, D. C., Almon, R. R., Pyszczynski, N. A. and Jusko, W. J. (2002a). "Pharmacodynamics and pharmacogenomics of methylprednisolone during 7-day infusions in rats." J Pharmacol Exp Ther 300(1): 245-56.

Ramakrishnan, R., DuBois, D. C., Almon, R. R., Pyszczynski, N. A. and Jusko, W. J. (2002b). "Fifth-generation model for corticosteroid pharmacodynamics: application to steady-state receptor down-regulation and enzyme induction patterns during seven-day continuous infusion of methylprednisolone in rats." $\underline{\mathrm{J}}$ Pharmacokinet Pharmacodyn 29(1): 1-24.

Randow, F., Syrbe, U., Meisel, C., Krausch, D., Zuckermann, H., Platzer, C., et al. (1995). "Mechanism of endotoxin desensitization: involvement of interleukin 10 and transforming growth factor beta." J Exp Med 181(5): 1887-92.

Rassias, A. J., Holzberger, P. T., Givan, A. L., Fahrner, S. L. and Yeager, M. P. (2005). "Decreased physiologic variability as a generalized response to human endotoxemia." Crit Care Med 33(3): 512-9.

Reyes, W. J., Brimioulle, S. and Vincent, J. L. (1999). "Septic shock without documented infection: an uncommon entity with a high mortality." Intensive Care Med 25(11): 1267-70.

Reynolds, A., Rubin, J., Clermont, G., Day, J., Vodovotz, Y. and Bard Ermentrout, G. (2006). "A reduced mathematical model of the acute inflammatory response: I. Derivation of model and analysis of anti-inflammation." J Theor Biol 242(1): 220-36.

Rhen, T. and Cidlowski, J. A. (2005). "Antiinflammatory action of glucocorticoids--new mechanisms for old drugs." N Engl J Med 353(16): 1711-23.

Richardson, R. P., Rhyne, C. D., Fong, Y., Hesse, D. G., Tracey, K. J., Marano, M. A., et al. (1989). "Peripheral blood leukocyte kinetics following in vivo lipopolysaccharide (LPS) administration to normal human subjects. Influence of elicited hormones and cytokines." Ann Surg 210(2): 239-45.

Riedemann, N. C., Guo, R. F. and Ward, P. A. (2003). "Novel strategies for the treatment of sepsis." Nat Med 9(5): 517-24.

Rifkind, D. (1967). "Prevention by polymyxin B of endotoxin lethality in mice." J Bacteriol 93(4): 1463-4.

Rittirsch, D., Flierl, M. A. and Ward, P. A. (2008). "Harmful molecular mechanisms in sepsis." Nat Rev Immunol 8(10): 776-87.

Romascin, A. D., Foster, D. M. and Marshall, J. C. (1998). "Let the Cells Speak: Neutrophils as Biologic Markers of the Inflammatory Response " Sepsis 2: 119125.

Rosenblueth, A. and Simeone, F. A. (1934). "The interrelations of vagal and accelerator effects on the cardiac rate " American Journal of Physiology 110: 42-55.

Saklatvala, J., Dean, J. and Clark, A. (2003). "Control of the expression of inflammatory response genes." Biochem Soc Symp(70): 95-106.

Sakurai, H., Suzuki, S., Kawasaki, N., Nakano, H., Okazaki, T., Chino, A., et al. (2003). "Tumor necrosis factor-alpha-induced IKK phosphorylation of NF-kappaB p65 on serine 536 is mediated through the TRAF2, TRAF5, and TAK1 signaling pathway." J Biol Chem 278(38): 36916-23.

Sama, A. E., D'Amore, J., Ward, M. F., Chen, G. and Wang, H. (2004). "Bench to bedside: HMGB1-a novel proinflammatory cytokine and potential therapeutic 
target for septic patients in the emergency department." Acad Emerg Med 11(8): $867-73$.

Sander, O., Welters, I. D., Foex, P. and Sear, J. W. (2005). "Impact of prolonged elevated heart rate on incidence of major cardiac events in critically ill patients with a high risk of cardiac complications." Crit Care Med 33(1): 81-8; discussion 241-2.

Sanderson, A. C. (1980). "Input-output analysis of an IPFM neural model: effects of spike regularity and record length." IEEE Trans Biomed Eng 27(3): 120-31.

Santman, F. W. (1992). "Catecholamines in critical care. The commonly used catecholamines: receptor and clinical profile, indications and dosages." Pharm Weekbl Sci 14(5): 290-6.

Santoni, D., Pedicini, M. and Castiglione, F. (2008). "Implementation of a regulatory gene network to simulate the TH1/2 differentiation in an agent-based model of hypersensitivity reactions." Bioinformatics 24(11): 1374-80.

Santos, A. A. and Wilmore, D. W. (1996). "The systemic inflammatory response: perspective of human endotoxemia." Shock 6 Suppl 1: S50-6.

Sato, N., Koeda, K., Ikeda, K., Kimura, Y., Aoki, K., Iwaya, T., et al. (2002). "Randomized study of the benefits of preoperative corticosteroid administration on the postoperative morbidity and cytokine response in patients undergoing surgery for esophageal cancer." Ann Surg 236(2): 184-90.

Scheinman, R. I., Cogswell, P. C., Lofquist, A. K. and Baldwin, A. S., Jr. (1995). "Role of transcriptional activation of I kappa B alpha in mediation of immunosuppression by glucocorticoids." Science 270(5234): 283-6.

Seely, A. J. and Christou, N. V. (2000). "Multiple organ dysfunction syndrome: exploring the paradigm of complex nonlinear systems." Crit Care Med 28(7): 2193-200.

Senftleben, U. (2003). "NF-kappa B in critical diseases: a bad guy?" Intensive Care Medicine 29(11): 1873-1876.

Sharshar, T., Hopkinson, N. S., Orlikowski, D. and Annane, D. (2005). "Science review: The brain in sepsis--culprit and victim." Crit Care 9(1): 37-44.

Shin, H. J., Lee, H., Park, J. D., Hyun, H. C., Sohn, H. O., Lee, D. W., et al. (2007). "Kinetics of binding of LPS to recombinant CD14, TLR4, and MD-2 proteins." Mol Cells 24(1): 119-24.

Singer, M., De Santis, V., Vitale, D. and Jeffcoate, W. (2004). "Multiorgan failure is an adaptive, endocrine-mediated, metabolic response to overwhelming systemic inflammation." Lancet 364(9433): 545-8.

Sontag, E. D. (2004). "Some new directions in control theory inspired by systems biology." Syst Biol (Stevenage) 1(1): 9-18.

Stein, P. K. and Kleiger, R. E. (1999). "Insights from the study of heart rate variability." Annu Rev Med 50: 249-61.

Storey, J. D., Xiao, W., Leek, J. T., Tompkins, R. G. and Davis, R. W. (2005). "Significance analysis of time course microarray experiments." Proc Natl Acad Sci U S A 102(36): 12837-42.

Suffredini, A. F., Fromm, R. E., Parker, M. M., Brenner, M., Kovacs, J. A., Wesley, R. A., et al. (1989). "The cardiovascular response of normal humans to the administration of endotoxin." N Engl J Med 321(5): 280-7. 
Suffredini, A. F., Hochstein, H. D. and McMahon, F. G. (1999). "Dose-related inflammatory effects of intravenous endotoxin in humans: evaluation of a new clinical lot of Escherichia coli O:113 endotoxin." J Infect Dis 179(5): 1278-82.

Sun, Y. N., DuBois, D. C., Almon, R. R. and Jusko, W. J. (1998). "Fourth-generation model for corticosteroid pharmacodynamics: a model for methylprednisolone effects on receptor/gene-mediated glucocorticoid receptor down-regulation and tyrosine aminotransferase induction in rat liver." J Pharmacokinet Biopharm 26(3): 289-317.

Sun, Y. N. and Jusko, W. J. (1998). "Transit compartments versus gamma distribution function to model signal transduction processes in pharmacodynamics." J Pharm Sci 87(6): 732-7.

Talwar, S., Munson, P. J., Barb, J., Fiuza, C., Cintron, A. P., Logun, C., et al. (2006). "Gene expression profiles of peripheral blood leukocytes after endotoxin challenge in humans." Physiol Genomics 25(2): 203-15.

Taveira da Silva, A. M., Kaulbach, H. C., Chuidian, F. S., Lambert, D. R., Suffredini, A. F. and Danner, A. F. (1993). "Shock and multiple-organ dysfunction after selfadministration of Salmonella endotoxin." N Engl J Med 328: 1457-1460.

Tetta, C., Fonsato, V., Ronco, C. and Camussi, G. (2005). "Recent insights into the pathogenesis of severe sepsis." Crit Care Resusc 7(1): 32-9.

Ting, A. Y. and Endy, D. (2002). "Signal transduction. Decoding NF-kappaB signaling." Science 298(5596): 1189-90.

Tong, W., Cao, X., Harris, S., Sun, H., Fang, H., Fuscoe, J., et al. (2003). "ArrayTrack-supporting toxicogenomic research at the U.S. Food and Drug Administration National Center for Toxicological Research." Environ Health Perspect 111(15): 1819-26.

Tschaikowsky, K., Schmidt, J. and Meisner, M. (1998). "Modulation of mouse endotoxin shock by inhibition of phosphatidylcholine-specific phospholipase C." $\underline{\mathrm{J}}$ Pharmacol Exp Ther 285(2): 800-4.

Uijtdehaage, S. H. and Thayer, J. F. (2000). "Accentuated antagonism in the control of human heart rate." Clin Auton Res 10(3): 107-10.

Van Amersfoort, E. S., Van Berkel, T. J. and Kuiper, J. (2003). "Receptors, mediators, and mechanisms involved in bacterial sepsis and septic shock." Clin Microbiol Rev 16(3): 379-414.

Van den Berghe, G. (2002). "Dynamic neuroendocrine responses to critical illness." Front Neuroendocrinol 23(4): 370-91.

van der Poll, T., Barber, A. E., Coyle, S. M. and Lowry, S. F. (1996). "Hypercortisolemia increases plasma interleukin-10 concentrations during human endotoxemia--a clinical research center study." J Clin Endocrinol Metab 81(10): 3604-6.

van der Poll, T., Coyle, S. M., Barbosa, K., Braxton, C. C. and Lowry, S. F. (1996). "Epinephrine inhibits tumor necrosis factor-alpha and potentiates interleukin 10 production during human endotoxemia." J Clin Invest 97(3): 713-9.

Van der Poll, T. and Lowry, S. F. (1997). "Epinephrine inhibits endotoxin-induced IL-1 beta production: roles of tumor necrosis factor-alpha and IL-10." Am J Physiol 273(6 Pt 2): R1885-90.

van der Poll, T. (2000). "Effects of Catecholamines on the Inflammatory Response." Sepsis 4: 159-167. 
van Deventer, S. J., Buller, H. R., ten Cate, J. W., Aarden, L. A., Hack, C. E. and Sturk, A. (1990). "Experimental endotoxemia in humans: analysis of cytokine release and coagulation, fibrinolytic, and complement pathways." Blood 76(12): 2520-6.

Van Zee, K. J., Coyle, S. M., Calvano, S. E., Oldenburg, H. S., Stiles, D. M., Pribble, J., et al. (1995). "Influence of IL-1 receptor blockade on the human response to endotoxemia." J Immunol 154(3): 1499-507.

Vedder, H., Schreiber, W., Yassouridis, A., Gudewill, S., Galanos, C. and Pollmacher, T. (1999). "Dose-dependence of bacterial lipopolysaccharide (LPS) effects on peak response and time course of the immune-endocrine host response in humans." Inflamm Res 48(2): 67-74.

Vemula, M., Berthiaume, F., Jayaraman, A. and Yarmush, M. L. (2004). "Expression profiling analysis of the metabolic and inflammatory changes following burn injury in rats." Physiol Genomics 18(1): 87-98.

Vincent, J. L. (2006). "Organ dysfunction in patients with severe sepsis." $\underline{\text { Surg Infect }}$ (Larchmt) 7 Suppl 2: S69-72.

Vincent, J. L. and Abraham, E. (2006). "The last 100 years of sepsis." Am J Respir Crit Care Med 173(3): 256-63.

Vodovotz, Y., Clermont, G., Chow, C. and An, G. (2004). "Mathematical models of the acute inflammatory response." Curr Opin Crit Care 10(5): 383-90.

Vodovotz, Y., Chow, C. C., Bartels, J., Lagoa, C., Prince, J. M., Levy, R. M., et al. (2006). "In silico models of acute inflammation in animals." Shock 26(3): 235-44.

Vodovotz, Y., Csete, M., Bartels, J., Chang, S. and An, G. (2008). "Translational systems biology of inflammation." PLoS Comput Biol 4(4): e1000014.

Vodovotz, Y., Constantine, G., Rubin, J., Csete, M., Voit, E. O. and An, G. (2009). "Mechanistic simulations of inflammation: current state and future prospects." Math Biosci 217(1): 1-10.

Vogel, T. R., Dombrovskiy, V. Y. and Lowry, S. F. (2009). "Trends in postoperative sepsis: are we improving outcomes?" Surg Infect (Larchmt) 10(1): 71-8.

Wang, H., Bloom, O., Zhang, M., Vishnubhakat, J. M., Ombrellino, M., Che, J., et al. (1999). "HMG-1 as a late mediator of endotoxin lethality in mice." Science 285(5425): 248-51.

Warner, H. R. and Cox, A. (1962). "A mathematical model of heart rate control by sympathetic and vagus efferent information." J Appl Physiol 17: 349-55.

Watson, J. D. and Crick, F. H. (1953). "Molecular structure of nucleic acids; a structure for deoxyribose nucleic acid." Nature 171(4356): 737-8.

Webster, J. I., Tonelli, L. and Sternberg, E. M. (2002). "Neuroendocrine regulation of immunity." Annu Rev Immunol 20: 125-63.

Wells, C. A., Ravasi, T. and Hume, D. A. (2005). "Inflammation suppressor genes: please switch out all the lights." J Leukoc Biol 78(1): 9-13.

Winchell, R. J. and Hoyt, D. B. (1996). "Spectral analysis of heart rate variability in the ICU: a measure of autonomic function." J Surg Res 63(1): 11-6.

Winchell, R. J. and Hoyt, D. B. (1997). "Analysis of heart-rate variability: a noninvasive predictor of death and poor outcome in patients with severe head injury." $\underline{\mathrm{J}}$ Trauma 43(6): 927-33. 
Winrow, V. R., Winyard, P. G., Morris, C. J. and Blake, D. R. (1993). "Free radicals in inflammation: second messengers and mediators of tissue destruction." $\mathrm{Br}$ Med Bull 49(3): 506-22.

Wittebole, X., Hahm, S., Coyle, S. M., Kumar, A., Calvano, S. E. and Lowry, S. F. (2007). "Nicotine exposure alters in vivo human responses to endotoxin." $\underline{\text { Clin }}$ Exp Immunol 147(1): 28-34.

Wysocka, M., Robertson, S., Riemann, H., Caamano, J., Hunter, C., Mackiewicz, A., et al. (2001). "IL-12 suppression during experimental endotoxin tolerance: dendritic cell loss and macrophage hyporesponsiveness." J Immunol 166(12): 7504-13.

Xing, Z., Jordana, M., Kirpalani, H., Driscoll, K. E., Schall, T. J. and Gauldie, J. (1994). "Cytokine expression by neutrophils and macrophages in vivo: endotoxin induces tumor necrosis factor-alpha, macrophage inflammatory protein-2, interleukin-1 beta, and interleukin-6 but not RANTES or transforming growth factor-beta 1 mRNA expression in acute lung inflammation." Am J Respir Cell Mol Biol 10(2): 148-53.

Xiong, W. and Ferrell, J. E., Jr. (2003). "A positive-feedback-based bistable 'memory module' that governs a cell fate decision." Nature 426(6965): 460-5.

Xu, Z. X., Sun, Y. N., DuBois, D. C., Almon, R. R. and Jusko, W. J. (1995). "Thirdgeneration model for corticosteroid pharmacodynamics: roles of glucocorticoid receptor mRNA and tyrosine aminotransferase mRNA in rat liver." $\underline{J}$ Pharmacokinet Biopharm 23(2): 163-81.

Yamakawa, T., Eguchi, S., Matsumoto, T., Yamakawa, Y., Numaguchi, K., Miyata, I., et al. (1999). "Intracellular signaling in rat cultured vascular smooth muscle cells: roles of nuclear factor-kappaB and p38 mitogen-activated protein kinase on tumor necrosis factor-alpha production." Endocrinology 140(8): 3562-72.

Yang, E., Maguire, T., Yarmush, M. L., Berthiaume, F. and Androulakis, I. P. (2007). "Bioinformatics analysis of the early inflammatory response in a rat thermal injury model." BMC Bioinformatics 8(1): 10.

Yang, E., Almon, R. R., Dubois, D. C., Jusko, W. J. and Androulakis, I. P. (2008). "Extracting global system dynamics of corticosteroid genomic effects in rat liver." J Pharmacol Exp Ther 324(3): 1243-54.

Yang, E., Almon, R. R., DuBois, D. C., Jusko, W. J. and Androulakis, I. P. (2009a). "Identification of global transcriptional dynamics." PLoS ONE accepted for publication.

Yang, E. H., Almon, R. R., Dubois, D. C., Jusko, W. J. and Androulakis, I. P. (2009b). "Identification of global transcriptional dynamics." PLoS One 4(7): e5992.

Yao, Z., Hoffman, E. P., Ghimbovschi, S., Dubois, D. C., Almon, R. R. and Jusko, W. J. (2008). "Mathematical modeling of corticosteroid pharmacogenomics in rat muscle following acute and chronic methylprednisolone dosing." Mol Pharm 5(2): 328-39.

Zamir, E. and Bastiaens, P. I. (2008). "Reverse engineering intracellular biochemical networks." Nat Chem Biol 4(11): 643-7.

Zaza, A. and Lombardi, F. (2001). "Autonomic indexes based on the analysis of heart rate variability: a view from the sinus node." Cardiovasc Res 50(3): 434-42. 
Zhang, P., Chan, J., Dragoi, A. M., Gong, X., Ivanov, S., Li, Z. W., et al. (2005). "Activation of IKK by thymosin alphal requires the TRAF6 signalling pathway." EMBO Rep 6(6): 531-7.

Zhou, Y., Lin, G., Baarsch, M. J., Scamurra, R. W. and Murtaugh, M. P. (1994). "Interleukin-4 suppresses inflammatory cytokine gene transcription in porcine macrophages." J Leukoc Biol 56(4): 507-13.

Zurakowski, R. and Teel, A. R. (2006). "A model predictive control based scheduling method for HIV therapy." J Theor Biol 238(2): 368-82.

Zwietering, M. H., Jongenburger, I., Rombouts, F. M. and van 't Riet, K. (1990). "Modeling of the Bacterial Growth Curve." Appl Environ Microbiol 56(6): 18751881. 


\title{
Curriculum Vitae
}

\author{
Panagiota T. Foteinou
}

\section{Education}
$06 / 2010-$
Postdoctoral Research Fellow, Chemical Engineering Department, University of California, Santa Barbara
$09 / 2005-05 / 2010$
PhD, Biomedical Engineering Department, Rutgers University - University of Medicine and Dentistry of New Jersey
$10 / 1999-11 / 2004$
Dipl. Ing. Degree in Chemical Engineering, Aristotle University of Thessaloniki, Greece

\section{Referred Journal Publications}

- Foteinou P.T., Calvano S.E., Lowry S.F. and Androulakis I.P., A Multi-scale Model for the Assessment of Autonomic Dysfunction in Human Endotoxemia, Phys Genomics doi: 10.1152/physiolgenomics.00184.2009 (2010)

- Dong X., Foteinou P.T., Calvano S.E., Lowry S.F. and Androulakis I.P., Agent-based Modeling of Endotoxin Induced Acute Inflammatory Response in Macrophages, PLoS ONE, 5(2):e9249 (2010)

- Foteinou P.T., Calvano S.E., Lowry S.F. and Androulakis I.P., A Multiscale Model of for the Assessment of Autonomic Dysfunction in Human Endotoxemia, Journal of Critical Care, 24(3): e25 (2009) (abstract)

- Foteinou P.T., Calvano S.E., Lowry S.F. and Androulakis I.P., In Silico Simulation of Corticosteroids Effect on an NFkB Dependent Physicochemical Model of Systemic Inflammation, PLoS ONE, 4(3): e4706 (2009)

- Foteinou P.T., Yang E. and Androulakis I.P., Networks, Biology and Systems Engineering: A Case Study in Inflammation, Comp. \& Chem. Eng, 33(12): 2028-2041 (2009) 
- Foteinou P.T., Yang E., Saharidis G.K., Ierapetritou M.G., and Androulakis I.P., A Mixed-integer Optimization Framework for the Synthesis and Analysis of Regulatory Networks, Journal of Global Optimization, 43 (2):263-276 (2009)

- Foteinou P.T., Calvano S.E., Lowry S.F. and Androulakis I.P., Modeling Endotoxin-Induced Systemic Inflammation Using an Indirect Response Approach, Math. Biosciences, 217 (1):27-42 (2009)

- Foteinou P.T., Calvano S.E., Lowry S.F. and Androulakis I.P., Translational Potential of Systems-based Models of Inflammation, Clinical and Translational Science, 2(1): 85-89 (2009)

- Foteinou P.T., Calvano S.E., Lowry S.F. and Androulakis I.P., An Indirect Response Model of Endotoxin-induced Systemic Inflammation, Journal of Critical Care, 22 (4):337-338 (2007) (abstract)

- Yang E., Foteinou P.T., King K.R., Yarmush M.L., Androulakis I.P., A Novel Non-overlapping Bi-clustering Algorithm for Network Generation using Living Cell Array Data. Bioinformatics 23:2306-2313 (2007) 